\title{
A family of 512 reverse order laws for generalized inverses of two matrix product: a review \\ Yongge Tian
}

\author{
CBE, Shanghai Business School, Shanghai, China
}

\begin{abstract}
Reverse order laws for generalized inverses of matrix products is a classic object of study in the theory of generalized inverses. One of the well-known reverse order laws for a matrix product $A B$ is $(A B)^{(i, \ldots, j)}=B^{\left(s_{2}, \ldots, t_{2}\right)} A^{\left(s_{1}, \ldots, t_{1}\right)}$, where $(\cdot)^{(i, \ldots, j)}$ denotes an $\{i, \ldots, j\}$-generalized inverse of matrix. Because $\{i, \ldots, j\}$-generalized inverse of a singular matrix is unique, the relationships between both sides of the reverse order law can be divided into four situations for consideration. This paper provides a thorough coverage of the reverse order laws for $\{i, \ldots, j\}$-generalized inverses of $A B$, from the development of background and preliminary tools to the collection of miscellaneous formulas and facts on the reverse order laws in one place with cogent introduction and references for further study. We begin with the introduction of a linear mixed model $y=A B \beta+A \gamma+\epsilon$ and the presentation of two least-squares methodologies to estimate the fixed parameter vector $\beta$ in the model, and the description of connections between the two types of least-squares estimators and the reverse order laws for generalized inverses of $A B$. We then prepare some valued matrix analysis tools, including a general theory on linear or nonlinear matrix identities, a group of expansion formulas for calculating ranks of block matrices, two groups of explicit formulas for calculating the maximum and minimum ranks of $B^{\left(s_{2}, \ldots, t_{2}\right)} A^{\left(s_{1}, \ldots, t_{1}\right)}$, as well as necessary and sufficient conditions for $B^{\left(s_{2}, \ldots, t_{2}\right)} A^{\left(s_{1}, \ldots, t_{1}\right)}$ to be invariant with respect to the choice of $B^{\left(s_{2}, \ldots, t_{2}\right)} A^{\left(s_{1}, \ldots, t_{1}\right)}$. We then present a unified approach to the 512 matrix set inclusion problems associated with the above reverse order laws for the eight commonly-used types of generalized inverses of $A, B$, and $A B$ through use of the definitions of generalized inverses, the block matrix method (BMM), the matrix rank method (MRM), the matrix equation method (MEM), and various algebraic calculations of matrices.
\end{abstract}

Keywords: matrix product; orthogonal projector; generalized inverse; reverse order law; BMM; MEM; MRM

Mathematics Subject Classifications: 15A03; 15A09; 15A24; 47A05

\section{Introduction}

We begin with introducing the notation adopted in this paper. Let $\mathbb{C}^{m \times n}$ denote the collection of all $m \times n$ complex matrices; $r(A), \mathscr{R}(A)$, and $\mathscr{N}(A)$ denote the rank, the range, and the null space of a matrix $A \in \mathbb{C}^{m \times n}$, respectively; $I_{m}$ denote the identity matrix of order $m$; and $[A, B]$ denote a row block matrix consisting of $A$ and $B$. The Moore-Penrose inverse of $A \in \mathbb{C}^{m \times n}$, denoted by $A^{\dagger}$, is the unique matrix $X \in \mathbb{C}^{n \times m}$ satisfying the four Penrose equations

$$
\text { (i) } A X A=A \text {, (ii) } X A X=X \text {, (iii) }(A X)^{*}=A X, \text { (iv) }(X A)^{*}=X A \text {. }
$$

A matrix $X$ is called an $\{i, \ldots, j\}$-generalized inverse of $A$, denoted by $A^{(i, \ldots, j)}$, if it satisfies the $i$ th, $\ldots, j$ th equations in (1.1). The collection of all $\{i, \ldots, j\}$-generalized inverses of $A$ is denoted by $\left\{A^{(i, \ldots, j)}\right\}$. There are all 15 types of $\{i, \ldots, j\}$-generalized inverses of $A$ by definition, but people are mainly interested in the following eight situations that involve the first equation:

$$
A^{\dagger}, \quad A^{(1,3,4)}, \quad A^{(1,2,4)}, \quad A^{(1,2,3)}, \quad A^{(1,4)}, \quad A^{(1,3)}, \quad A^{(1,2)}, \quad A^{(1)},
$$

which are usually called the eight commonly-used types of generalized inverses of $A$ in the literature; see e.g., $[16,19,57]$. In addition, let $P_{A}=A A^{\dagger}, E_{A}=I_{m}-A A^{\dagger}$, and $F_{A}=I_{n}-A^{\dagger} A$, denote the three orthogonal projectors (Hermitian idempotent matrices) induced from $A$.

One of the most important applications of generalized inverses is to deal with singular matrices and their algebraic operations that occur in mathematics and applications. To emphasize the occurrence of generalized inverses in matrix calculations, we can generally write matrix expressions that involve a family of generalized inverses $A_{1}^{\left(i_{1}, \ldots, j_{1}\right)}, A_{2}^{\left(i_{2}, \ldots, j_{2}\right)}, \ldots, A_{k}^{\left(i_{k}, \ldots, j_{k}\right)}$ as

$$
f\left(A_{1}^{\left(i_{1}, \ldots, j_{1}\right)}, A_{2}^{\left(i_{2}, \ldots, j_{2}\right)}, \ldots, A_{k}^{\left(i_{k}, \ldots, j_{k}\right)}\right),
$$

E-mail: yongge.tian@gmail.com 
where $f(\cdot)$ denotes certain algebraic operations of matrices. We also denote the collection of matrix values of the function with respect to all possible choices of the generalized inverses by

$$
\mathcal{D}_{f}=\left\{f\left(A_{1}^{\left(i_{1}, \ldots, j_{1}\right)}, A_{2}^{\left(i_{2}, \ldots, j_{2}\right)}, \ldots, A_{k}^{\left(i_{k}, \ldots, j_{k}\right)}\right)\right\}
$$

and called it the domain of 1.3. As is known to all, one of the fundamental tasks in algebra is to establish and describe various algebraic equalities for operations of elements in the algebra. In the theory of generalized inverses of matrices, such a task is designated as the derivation of equalities that involve matrices and their generalized inverses. Using the above notation, we can write the equalities in the following general form

$$
f\left(A_{1}^{\left(i_{1}, \ldots, j_{1}\right)}, A_{2}^{\left(i_{2}, \ldots, j_{2}\right)}, \ldots, A_{k}^{\left(i_{k}, \ldots, j_{k}\right)}\right)=g\left(B_{1}^{\left(s_{1}, \ldots, t_{1}\right)}, B_{2}^{\left(s_{2}, \ldots, t_{2}\right)}, \ldots, B_{l}^{\left(s_{l}, \ldots, t_{l}\right)}\right)
$$

for two different matrix expressions. Because the matrix values of $f(\cdot)$ and $g(\cdot)$ are not necessarily unique, we can divide the relationships between the both sides of 1.5 into the following four situations

$$
\mathcal{D}_{f} \cap \mathcal{D}_{g} \neq \emptyset, \quad \mathcal{D}_{f} \supseteq \mathcal{D}_{g}, \quad \mathcal{D}_{f} \subseteq \mathcal{D}_{g}, \quad \mathcal{D}_{f}=\mathcal{D}_{g}
$$

by means of the domain notation in (1.4). There are many examples of $(1.5)$ and $(1.6)$ that occur in the theory of generalized inverses and their applications. One of the most popular class of (1.5) and (1.6) are concerned with equalities of products of generalized inverses. Recall that if $A$ and $B$ are two nonsingular matrices of the same size, the product $A B$ is nonsingular as well, and the inverse of the product $A B$ can be expressed as $(A B)^{-1}=B^{-1} A^{-1}$, which is usually called the two-term reverse order law (ROL) for the standard inverse of a matrix product. An extension of the ROL to the multiple matrix product case is given by $\left(A_{1} A_{2} \cdots A_{d}\right)^{-1}=A_{d}^{-1} \cdots A_{2}^{-1} A_{1}^{-1}$. These ROLs are best-known fundamental identities in matrix algebra, which can be used to simplify matrix expressions that involve inverse operations of nonsingular matrix products. If a given matrix product is singular, generalized inverses of the product can also be written as certain reverse order products of generalized inverses of the given matrices. To take the most useful case, we assume that $A \in \mathbb{C}^{m \times n}$ and $B \in \mathbb{C}^{n \times p}$ are two given matrices. Then the product $A B \in \mathbb{C}^{m \times p}$ is defined, but generally it is singular. In this situation, an extension of $(A B)^{-1}=B^{-1} A^{-1}$ to generalized inverses of $A B$ can be written as the following two-term ROL:

$$
(A B)^{(i, \ldots, j)}=B^{\left(s_{2}, \ldots, t_{2}\right)} A^{\left(s_{1}, \ldots, t_{1}\right)},
$$

which is obviously a special case of (1.4). Because the non-commutativity of matrix algebra, and also because $A A^{(s, \ldots, t)} \neq I_{m}, A^{(s, \ldots, t)} A \neq I_{n}, B B^{\left(s_{2}, \ldots, t_{2}\right)} \neq I_{n}$, and $B^{\left(s_{2}, \ldots, t_{2}\right)} B \neq I_{p}$ for two singular matrices $A$ and $B$, the reverse-order product $B^{\left(s_{2}, \ldots, t_{2}\right)} A^{(s, \ldots, t)}$ on the right-hand side of 1.7$)$ does not necessarily satisfy the matrix equations defined for $(A B)^{(i, \ldots, j)}$. In this case, we denote by $\left\{(A B)^{(i, \ldots, j)}\right\}$ and $\left\{B^{\left(s_{2}, \ldots, t_{2}\right)} A^{(s, \ldots, t)}\right\}$ the collections of all possible choices of the matrices on both sides of (1.7), so that it is natural to divide (1.7) into the following four reasonable relationships for the two matrix sets

$$
\begin{aligned}
& \left\{(A B)^{(i, \ldots, j)}\right\} \cap\left\{B^{\left(s_{2}, \ldots, t_{2}\right)} A^{\left(s_{1}, \ldots, t_{1}\right)}\right\} \neq\{\emptyset\}, \\
& \left\{(A B)^{(i, \ldots, j)}\right\} \supseteq\left\{B^{\left(s_{2}, \ldots, t_{2}\right)} A^{\left(s_{1}, \ldots, t_{1}\right)}\right\}, \\
& \left\{(A B)^{(i, \ldots, j)}\right\} \subseteq\left\{B^{\left(s_{2}, \ldots, t_{2}\right)} A^{\left(s_{1}, \ldots, t_{1}\right)}\right\}, \\
& \left\{(A B)^{(i, \ldots, j)}\right\}=\left\{B^{\left(s_{2}, \ldots, t_{2}\right)} A^{\left(s_{1}, \ldots, t_{1}\right)}\right\} .
\end{aligned}
$$

Because generalized inverses of a matrix are defined to be common solutions of certain matrix equations, the ROL problems are in fact to describe connections among matrix expressions composed by solutions of several matrix equations.

Eqs. 1.8 - 1.11) do not necessarily hold for different choices of generalized inverses of the matrices. Thus we wish to find identifying conditions for (1.8)-(1.11) to hold under various assumptions. This is really a tremendous work because there are all 15 types of $\{i, \ldots, j\}$-generalized inverse for a given matrix according to combinatoric choices of the four Penrose equations. Eqs. (1.8)-(1.11) and their extensions to multiple matrix products have been a classic objects of study in the thenory of generalized inverses and applications, and have attracted considerable attention since 1960s. Literature on reverse order product of generalized inverses of matrix products is abundant,

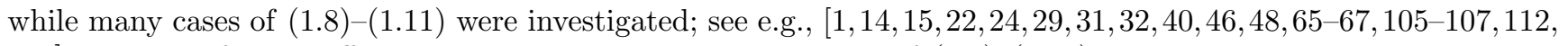
113. In spite of many efforts, people consider only a small part of $(1.8)-(1.11)$ in the past several decades, while a large body of these ROLs remain unresolved.

After (1.8)-(1.11) are formulated, a huge task underlying is to establish necessary and sufficient conditions for the equalities to hold (for the matrix equations to be solvable), but one can often be left in a confounding place of techniques, philosophies and nuance when approaching so many different equalities. We now realize that a sufficient resolution of this kind of matrix equality problems is relying on the two elementary but strong matrix analytic 
tools - the matrix rank method (MRM) and the block matrix method (BMM), which can help us to describe and prove the equalities in a clear and concise way. In fact, these two methodologies have been welcomed as most efficient and popular analytical techniques in matrix calculus.

We next give a specified introduction to the theory of matrix ranks. The rank of matrix is one of the basic concepts in linear algebra, which can be defined by different manners and can be calculated directly by transforming the matrix to certain row and/or column echelon forms. As one of the key indicators of a matrix, one can use the rank of matrix to describe the performance of the matrix under general assumptions, such as, the nullity, singularity, and nonsingularity of the matrix, as well as the dimension of the row or column space of the matrix, numbers of singular values, etc. There are many simple and interesting properties on the rank of a matrix, a best-known fact is: $A=0 \Leftrightarrow r(A)=0$. Also note the rule $A=B \Leftrightarrow A-B=0$ for any two matrices $A$ and $B$ of the same sizes, through which it is possible to transform the equality preserving the equivalence. Thus, we have the rule $A=B \Leftrightarrow r(A-B)=0$. Furthermore, assume that $\mathcal{S}_{1}$ and $\mathcal{S}_{2}$ are two sets consisting of matrices of the same size. Then it is straightforward to see from the above fact that the following two pairs of equivalent statements hold

$$
\begin{aligned}
& \mathcal{S}_{1} \cap \mathcal{S}_{2} \neq \emptyset \Leftrightarrow \min _{A \in \mathcal{S}_{1}, B \in \mathcal{S}_{2}} r(A-B)=0, \\
& \mathcal{S}_{1} \subseteq \mathcal{S}_{2} \Leftrightarrow \max _{A \in \mathcal{S}_{1}} \min _{B \in \mathcal{S}_{2}} r(A-B)=0 .
\end{aligned}
$$

Because of the simplicity of the concept of matrix rank, one can easily understand the meaning of the above assertions, and would like to take them as a useful way of characterizing connections of two matrices, as well as matrix equalities. To speak precisely, if certain analytical formulas for calculating the rank of a difference $A-B$ are established, we can obtain from the formulas some algebraic properties of the difference as described above, as well as necessary and sufficient conditions for $A=B$ to hold. It has been luckily noticed from many well-known rank equalities and inequalities that there do exist general ways of establishing nontrivial expansion formulas for calculating ranks of matrix expressions, and thus the matrix rank method described above is a meaningful and available technique to characterize matrix equalities. It has been realized in the past several decades that the rank of matrix is striking tool in the investigation of matrix expressions and their connections. This tool is based on establishing various algebraic expansion formulas for calculating ranks of given matrix expressions under various assumptions, so that it is named as the matrix rank method for the sake of convenience. In fact, the MRM has extensively been utilized to describe algebraic properties of matrix expressions and to establish matrix equalities that involve inverses and generalized inverses of matrices since the seminal work in [51]. In the past several decades, the present author paid a great attention to the development of the MRM in matrix theory and solved many fundamental problems in the theory of generalized inverses of matrices through use of the MRM, including the simplification and derivations of various complicated and nuanced matrix expressions and equalities, the establishments and characterizations of various ROLs for generalized inverses of matrix products, establishment of many closed-form formulas for calculating ranks of block matrices, sums and difference of matrices etc., see e.g., $73,75,78,79,81,82,84,88,94,95,98,100$ for a variety of detailed contributions in this respect. In addition, various rank maximization and minimization problems of matrices have been formulated in other disciplines of mathematics and applications and has been expanding in many directions during the last two decades; see e.g., $30,42,50,52,60,116$ among others. Perhaps, no methods in linear algebra and matrix theory, as described above, are more elementary and straightforward than the MRM in characterization of matrix equalities.

The main purpose of this paper is to gather in a single document various known and novel formulas and facts on a family of 512 one-sided set inclusions associated with 1.9 for the eight commonly-used types of generalized inverses of $A, B$, and $A B$ through use of the definitions of generalized inverses, the block matrix method, the matrix equation method, and the matrix rank method. In fact, it has been realized since 1970s that these the three methods are powerful tools to characterize matrix equalities that involve generalized inverses, while a seminal work on applications of these methods in the theory of generalized inverses was presented in [51]. In the past several decades, the present author introduced these methods in the investigation of ROLs and other matrix equalities and established thousands of results and facts on this topic; see e.g., $72,73,76-78,80-84,86,87,90,93,95,98,101]$.

This paper is organized as follows. In Section 2, we go through some basic facts of the ordinary least-squares estimators (OLSEs) for unknown parameters in a two-level linear regression, and establish some analytical formulas for calculating the OLSEs, as well as explicit expressions of the expectations and the covariance matrices of the OLSEs by means of generalized inverses of the given vectors and matrices. We then describe some mathematical equivalences between some equalities of the OLSEs and ROLs for generalized inverses of the products of the given matrices in the model. The core work of this paper is to present a classification analysis to a group of 512 one-sided matrix set inclusions associated with (1.7). To finish this task, we introduce in Section 3 various fundamental formulas of generalized inverses of matrices and their products, in Section 4 we provide various formulas for calculating ranks of matrices and their generalized inverses, and in Section 5 we present a group of results on linear and multilinear matrix identities that involve one or multiple variable matrices. In Section 6, we discuss the 
invariance property of matrix products that involve two generalized inverses. In Section 7, we present a family of fundamental equalities for generalized inverses of two matrices of same size. In Section 8, we present 126 known analytical formulas for calculating the maximum and minimum ranks of $B^{\left(s_{2}, \ldots, t_{2}\right)} A^{(s, \ldots, t)}$ with respect to the choice of the generalized inverses, and give several groups of conclusions on the invariance property of the preceding matrix products with respect to the choice of the generalized inverses. A variety of necessary and sufficient conditions for (1.7) to hold are presented in Sections 9, 10, and 11, respectively. Remarks and a list of challenging open problems are presented in Section 12. For conciseness, we omit straightforward proofs or provide just one proof of a set of similar results and facts.

\section{A least-squares estimation problem related to ROLs in linear sta- tistical models}

It is well known that parametric regression analysis is perhaps the most commonly employed tool in statistical data analysis and inference, while linear regression models belong to classic issues in statistical theory and are the common roots of many branches of current statistical theory. Although there has been a relatively systematical research about linear regression models and their applications in the past centuries, one can still propose many theoretical problems in this field and investigate these problems by way of various mathematical analysis tools. When using linear regression models to fit given data, unknown parameters in the models may not necessarily be assumed to be fixed, instead, to vary at more than one level, or to be given in nested forms. Multi-level hierarchical linear model is a feasible technique of fitting data that have a hierarchical structure. Because of the occurrence of parameters at more than one level, the inference of a multi-level hierarchical linear model involves various nested calculations of given matrices and vectors in the model. In fact, many problems in statistics and applications involve analyzing and manipulating this kind of nested structured data and models; see a number of books including $25,26,33,43,49,55,59,64,104,111]$. In this paper, we consider a two-level hierarchical linear model defined by

$$
\mathscr{M}:\left\{\begin{array}{l}
y=A \alpha+\epsilon, \alpha=B \beta+\gamma, E(\epsilon)=0, E(\gamma)=0, \\
\operatorname{Cov}(\epsilon)=\sigma^{2} I_{n}, \operatorname{Cov}(\gamma)=\tau^{2} I_{p}, \operatorname{Cov}(\epsilon, \gamma)=0,
\end{array}\right.
$$

where in the first-level model, $y \in \mathbb{R}^{n \times 1}$ is a vector of observable response variables, $A \in \mathbb{R}^{n \times p}$ is a known matrix of arbitrary rank, $\alpha \in \mathbb{R}^{p \times 1}$ is a vector of unobservable random variables, $\epsilon \in \mathbb{R}^{n \times 1}$ is a vector of randomly distributed error terms, $\sigma^{2}$ is an arbitrary positive scaling factor; in the second-level model, $B \in \mathbb{R}^{p \times k}$ is a known matrix of arbitrary rank, $\beta \in \mathbb{R}^{k \times 1}$ is a vector of fixed but unknown parameters, $\gamma \in \mathbb{R}^{p \times 1}$ is a vector of unobservable random variables, $\tau^{2}$ is an arbitrary positive scaling factor. This kind of models have different names in statistical analysis according to their origination, such as, random-effect models, hierarchical models, nested models, etc. Substituting the second equation in 2.1) into the first equation leads to the following linear mixed model

$$
\mathscr{N}:\left\{\begin{array}{l}
y=A B \beta+A \gamma+\epsilon \\
E(y)=A B \beta, \operatorname{Cov}(y)=\sigma^{2} I_{n}+\tau^{2} A A^{T} .
\end{array}\right.
$$

It is well known that the most common technique used to estimate the unknown parameters in linear regression models is the method of least-squares. Because of the two alternative forms $\mathscr{M}$ and $\mathscr{N}$, there exist in fact two kinds of the ordinary least-squares estimator (OLSE) for the unknown parameter vector $\beta$ in $\mathscr{M}$ and $\mathscr{N}$. This fact prompt us to discuss the connections between the OLSEs from mathematical and statistical points of view.

Since there are two alternative forms in (2.1) and $(2.2)$, respectively, we are able to adopt different procedures to calculate the OLSEs of the unknown parameter vector $\beta$ and the mean vector $A B \beta$ in (2.1) and (2.2) as follows.

(I) The standard method is to

$$
\text { minimize }(y-A B \beta)^{T}(y-A B \beta)
$$

in the context of (2.2). It is easy to verify that the norm $(y-A B \beta)^{T}(y-A B \beta)$ in $(2.3)$ can be decomposed as

$$
(y-A B \beta)^{T}(y-A B \beta)=y^{T} E_{A B} y+\left(P_{A B} y-A B \beta\right)^{T}\left(P_{A B} y-A B \beta\right),
$$

where the two terms on the right-hand side satisfy $y^{T} E_{A B} y \geqslant 0$ and $\left(P_{A B} y-A B \beta\right)^{T}\left(P_{A B} y-A B \beta\right) \geqslant 0$; see 96, 97. Hence,

$$
\min _{\beta \in \mathbb{R}^{p \times 1}}(y-A B \beta)^{T}(y-A B \beta)=y^{T} E_{A B} y+\min _{\beta \in \mathbb{R}^{p \times 1}}\left(P_{A B} y-A B \beta\right)^{T}\left(P_{A B} y-A B \beta\right)=y^{T} E_{A B} y,
$$


where the equation $A B \beta=A B(A B)^{\dagger} y$, which is equivalent to the normal equation $(A B)^{T} A B \beta=A B^{T} y$ by pre-multiplying $(A B)^{T}$, is always consistent; see e.g., [34, p. 114] and 63, pp. 164-165]. Solving the equation gives the well-known OLSEs of $\beta$ and $A B \beta$ under $\mathscr{N}$ :

$$
\begin{aligned}
& \operatorname{OLSE}_{\mathscr{N}}(\beta)=\left[(A B)^{\dagger}+F_{A B} U\right] y=(A B)^{(1,3)} y, \\
& \operatorname{OLSE}_{\mathscr{N}}(A B \beta)=A B \operatorname{OLSE}_{\mathscr{N}}(\beta)=A B(A B)^{(1,3)} y,
\end{aligned}
$$

where $U$ is an arbitrary matrix. Furthermore, the expectations and the covariance matrices of OLSE $\mathscr{N}(\beta)$ and $\mathrm{OLSE}_{\mathscr{N}}(A B \beta)$ are given by

$$
\begin{aligned}
& E\left[\operatorname{OLSE}_{\mathscr{N}}(\beta)\right]=(A B)^{\dagger} A B \beta, \\
& \operatorname{Cov}\left[\operatorname{OLSE}_{\mathscr{N}}(\beta)\right]=(A B)^{\dagger}\left(\sigma^{2} I_{n}+\tau^{2} A A^{T}\right)\left[(A B)^{\dagger}\right]^{T}, \\
& E\left[\operatorname{OLSE}{ }_{\mathscr{N}}(A B \beta)\right]=A B \beta, \\
& \operatorname{Cov}\left[\operatorname{OLSE}{ }_{\mathscr{N}}(A B \beta)\right]=A B(A B)^{\dagger}\left(\sigma^{2} I_{n}+\tau^{2} A A^{T}\right) A B(A B)^{\dagger} .
\end{aligned}
$$

(II) On the other hand, we may first solve the least-squares problem $(y-A \alpha)^{T}(y-A \alpha)=$ min under $(2.1)$ and obtain the OLSE of $\alpha$ as follows

$$
\mathrm{OLSE}_{\mathscr{M}}(\alpha)=\left(A^{\dagger}+F_{A} U_{1}\right) y=A^{(1,3)} y
$$

where $U_{1}$ is an arbitrary matrix. Substituting this formula into the second equation in (2.1) yields

$$
A^{(1,3)} y=B \beta+\gamma
$$

In this case, solving $\left\|A^{(1,3)} y-B \beta\right\|^{2}=\min$ under 2.11 leads to

$$
\begin{aligned}
& \mathrm{OLSE}_{\mathscr{M}}(\beta)=\left(B^{\dagger}+F_{B} U_{2}\right) A^{(1,3)} y=B^{(1,3)} A^{(1,3)} y, \\
& \mathrm{OLSE}_{\mathscr{M}}(A B \beta)=A B B^{(1,3)} A^{(1,3)} y
\end{aligned}
$$

where $U_{2}$ is an arbitrary matrix. In the case of Moore-Penrose inverses, the expectations and the covariance matrices of these estimators are given by

$$
\begin{aligned}
& E[\mathrm{OLSE} \\
& \mathscr{M}(\beta)]=B^{\dagger} A^{\dagger} A B \beta, \\
& \operatorname{Cov}\left[\mathrm{OLSE}_{\mathscr{M}}(\beta)\right]=B^{\dagger} A^{\dagger}\left(\sigma^{2} I_{n}+\tau^{2} A A^{T}\right)\left(B^{\dagger} A^{\dagger}\right)^{T}, \\
& E[\mathrm{OLSE} \mathscr{M}(A B \beta)]=A B B^{\dagger} A^{\dagger} A B \beta, \\
& \operatorname{Cov}\left[\mathrm{OLSE}_{\mathscr{M}}(A B \beta)\right]=A B B^{\dagger} A^{\dagger}\left(\sigma^{2} I_{n}+\tau^{2} A A^{T}\right)\left(A B B^{\dagger} A^{\dagger}\right)^{T} .
\end{aligned}
$$

Note from 2.4 -2.9 and $2.12-2.17$ that the OLSEs under $\mathscr{N}$ and $\mathscr{M}$ are given in different formulas. Thus they have different performance, and it would be of interest to describe the relationships between the OLSEs under $\mathscr{N}$ and $\mathscr{M}$, in particular, it is necessary to establish identifying conditions for the following 6 equalities for the OLSEs and their expectations

$$
\begin{aligned}
& \operatorname{OLSE}_{\mathscr{M}}(\beta)=\operatorname{OLSE}_{\mathscr{N}}(\beta), \\
& E\left[\operatorname{OLSE}_{\mathscr{M}}(\beta)\right]=E\left[\operatorname{OLSE}_{\mathscr{N}}(\beta)\right] \\
& \operatorname{Cov}\left[\operatorname{OLSE}_{\mathscr{N}}(\beta)\right]=\operatorname{Cov}\left[\operatorname{OLSE}_{\mathscr{M}}(\beta)\right],
\end{aligned}
$$

and

$$
\begin{aligned}
& \operatorname{OLSE}_{\mathscr{M}}(A B \beta)=\mathrm{OLSE}_{\mathscr{N}}(A B \beta), \\
& E\left[\mathrm{OLSE}_{\mathscr{M}}(A B \beta)\right]=E\left[\operatorname{OLSE}_{\mathscr{N}}(A B \beta)\right], \\
& \operatorname{Cov}\left[\mathrm{OLSE}_{\mathscr{N}}(A B \beta)\right]=\operatorname{Cov}\left[\operatorname{OLSE}_{\mathscr{M}}(A B \beta)\right]
\end{aligned}
$$

to hold, respectively. It is clear that we need to compare the coefficient matrices of $y$ in (2.4) and (2.12), the expectations in (2.6), 2.8, 2.14, and 2.16) in order to examine the four equalities, and obtain the following facts. 
Lemma 2.1. Let the OLSEs of $\beta$ and $A B \beta$ in $\mathscr{M}$ and $\mathscr{N}$ be as given in (2.4), 2.5), (2.6), (2.8), (2.14), and (2.16), respectively. Then the following 6 assertions hold

$$
\begin{aligned}
& \operatorname{OLSE}_{\mathscr{M}}(\beta)=\operatorname{OLSE}_{\mathscr{N}}(\beta) \Longleftrightarrow(A B)^{\dagger}=B^{\dagger} A^{\dagger}, \\
& E\left[\operatorname{OLSE}_{\mathscr{M}}(\beta)\right]=E\left[\operatorname{OLSE}_{\mathscr{N}}(\beta)\right] \Longleftrightarrow(A B)^{\dagger} A B=B^{\dagger} A^{\dagger} A B, \\
& \operatorname{OLSE}_{\mathscr{M}}(A B \beta)=\operatorname{OLSE}_{\mathscr{N}}(A B \beta) \Longleftrightarrow A B(A B)^{\dagger}=A B B^{\dagger} A^{\dagger}, \\
& E\left[\operatorname{OLSE}_{\mathscr{M}}(A B \beta)\right]=E\left[\operatorname{OLSE}_{\mathscr{N}}(A B \beta)\right] \Longleftrightarrow A B=A B B^{\dagger} A^{\dagger} A B,
\end{aligned}
$$

and

$$
\begin{aligned}
& \operatorname{Cov}\left[\operatorname{OLSE}_{\mathscr{N}}(\beta)\right]=\operatorname{Cov}\left[\operatorname{OLSE}_{\mathscr{M}}(\beta)\right] \Leftrightarrow\left\{\begin{array}{l}
{\left[B^{T}\left(A^{T} A\right) B\right]^{\dagger}=B^{\dagger}\left(A^{T} A\right)^{\dagger}\left(B^{T}\right)^{\dagger} \text { and }} \\
(A B)^{\dagger} A A^{T}\left[(A B)^{\dagger}\right]^{T}=B^{\dagger} A^{\dagger} A\left(B^{\dagger}\right)^{T}
\end{array}\right. \\
& \operatorname{Cov}\left[\operatorname{OLSE}_{\mathscr{N}}(A B \beta)\right]=\operatorname{Cov}\left[\operatorname{OLSE}_{\mathscr{M}}(A B \beta)\right] \Leftrightarrow\left\{\begin{array}{c}
A B(A B)^{\dagger}=A B B^{\dagger} A^{\dagger}\left(A B B^{\dagger} A^{\dagger}\right)^{T} \text { and } \\
A B(A B)^{\dagger}\left(A A^{T}\right) A B(A B)^{\dagger}=\left(A B B^{\dagger}\right) A^{\dagger} A\left(A B B^{\dagger}\right)^{T} .
\end{array}\right.
\end{aligned}
$$

The matrix equality in 2.24 is the well-known ROL for the Moore-Penrose generalized inverses of the product $A B$, while the three matrix equalities in 2.25)-2.27) are obtained by pre- and post-multiplying the equality in (2.24) with $A B$, respectively. It is obvious that the four matrix equalities in (2.24)-(2.27) are algebraic issues in matrix mathematics. The equivalent statements in (2.24)-(2.27), however, show that the four matrix equalities in (2.24)-(2.27) can be used to describe and solve some fundamental problems on performance of OLSEs in statistical analysis of regression models, and therefore can be taken as remarkable motivation and valuable explanation for approaching various matrix equalities that involve generalized inverses. in mathematics and applications. It is should be pointed out that the four matrix equalities in (2.24)-(2.27) do not necessarily hold for two general matrices $A$ and $B$. Thus it is imperative to establish necessary and sufficient conditions for the four matrix equalities in (2.24)-(2.27) to hold in order to interpret and use the four statistical statements in (2.24)-(2.27).

\section{Fundamental formulas and facts about generalized inverses of ma- trices}

Facing the task of describing the relationships between the matrix sets in (1.8)-(1.11), we present in this section a brief introduction to the theory of generalized inverses of matrices, which we shall use to establish and simplify various matrix equalities that involve generalized inverses in the sequel. Note from the definitions of generalized inverses of a matrix that they are in fact defined to be (common) solutions of some matrix equations. Thus analytical expressions of generalized inverses of matrices can be written as certain matrix-valued functions with one or more variable matrices. In fact, analytical formulas of generalized inverses of matrices and their functions are important issues and tools in matrix analysis. For example, the basic formulas in the following lemma can be found, e.g., in $[16,19,57]$.

Lemma 3.1. Let $A \in \mathbb{C}^{m \times n}$. Then the following results hold.

(a) The following equalities hold

$$
\begin{aligned}
& \left(A^{\dagger}\right)^{*}=\left(A^{*}\right)^{\dagger},\left(A^{\dagger}\right)^{\dagger}=A,\left(A A^{*}\right)^{\dagger}=\left(A^{\dagger}\right)^{*} A^{\dagger},\left(A^{*} A\right)^{\dagger}=A^{\dagger}\left(A^{\dagger}\right)^{*}, \\
& A A^{\dagger}=\left(A A^{\dagger}\right)^{*}=\left(A^{*}\right)^{\dagger} A^{*}, A^{\dagger} A=\left(A^{\dagger} A\right)^{*}=A^{*}\left(A^{*}\right)^{\dagger}, \\
& A^{*}=\left(A A^{\dagger} A\right)^{*}=A^{*}\left(A^{*}\right)^{\dagger} A^{*},\left(A A^{*} A\right)^{\dagger}=A^{\dagger}\left(A^{\dagger}\right)^{*} A^{\dagger}, \\
& \mathscr{R}(A)=\mathscr{R}\left(A A^{*}\right)=\mathscr{R}\left(A A^{*} A\right)=\mathscr{R}\left(A A^{\dagger}\right)=\mathscr{R}\left[\left(A^{\dagger}\right)^{*}\right], \\
& \mathscr{R}\left(A^{*}\right)=\mathscr{R}\left(A^{*} A\right)=\mathscr{R}\left(A^{*} A A^{*}\right)=\mathscr{R}\left(A^{\dagger}\right)=\mathscr{R}\left(A^{\dagger} A\right), \\
& r(A)=r\left(A^{*}\right)=r\left(A^{\dagger}\right)=r\left(A A^{*}\right)=r\left(A^{*} A\right)=r\left(A A^{\dagger}\right)=r\left(A^{\dagger} A\right) .
\end{aligned}
$$

(b) The general expressions of the seven commonly-used types of generalized inverses $A^{(1,3,4)}, A^{(1,2,4)}, A^{(1,2,3)}$, $A^{(1,4)}, A^{(1,3)}, A^{(1,2)}$, and $A^{(1)}$ of $A$ can be written in the following 7 matrix-valued functions

$$
\begin{aligned}
& A^{(1,3,4)}=A^{\dagger}+F_{A} U E_{A}, \\
& A^{(1,2,4)}=A^{\dagger}+A^{\dagger} A U E_{A}, \\
& A^{(1,2,3)}=A^{\dagger}+F_{A} U A A^{\dagger}, \\
& A^{(1,4)}=A^{\dagger}+U E_{A},
\end{aligned}
$$




$$
\begin{aligned}
& A^{(1,3)}=A^{\dagger}+F_{A} U, \\
& A^{(1,2)}=\left(A^{\dagger}+F_{A} U_{1}\right) A\left(A^{\dagger}+U_{2} E_{A}\right), \\
& A^{(1)}=A^{\dagger}+F_{A} U_{1}+U_{2} E_{A},
\end{aligned}
$$

where $U, U_{1}, U_{2} \in \mathbb{C}^{n \times m}$ are arbitrary. In particular,

$$
\begin{aligned}
& A^{(1,3,4)} \text { is unique } \Leftrightarrow \text { either } r(A)=m \text { or } r(A)=n, \\
& A^{(1,2,4)} \text { is unique } \Leftrightarrow \text { either } A=0 \text { or } r(A)=m, \\
& A^{(1,2,3)} \text { is unique } \Leftrightarrow \text { either } A=0 \text { or } r(A)=n, \\
& A^{(1,4)} \text { is unique } \Leftrightarrow r(A)=m, \\
& A^{(1,3)} \text { is unique } \Leftrightarrow r(A)=n, \\
& A^{(1,2)} \text { is unique } \Leftrightarrow \text { either } A=0 \text { or } r(A)=m=n, \\
& A^{(1)} \text { is unique } \Leftrightarrow r(A)=m=n, \text { namely, } A \text { is nonsingular. }
\end{aligned}
$$

If $r(A)=m$, the general expressions of the last seven generalized inverses of $A$ in $(1.2)$ can be written as

$$
A^{(1,3,4)}=A^{(1,2,4)}=A^{(1,4)}=A^{\dagger}, \quad A^{(1,2,3)}=A^{(1,3)}=A^{(1,2)}=A^{(1)}=A^{\dagger}+F_{A} V,
$$

where $V \in \mathbb{C}^{n \times m}$ is arbitrary. If $r(A)=n$, the general expressions of the last seven generalized inverses of $A$ in (1.2) can be written as

$$
A^{(1,3,4)}=A^{(1,2,3)}=A^{(1,3)}=A^{\dagger}, \quad A^{(1,2,4)}=A^{(1,4)}=A^{(1,2)}=A^{(1)}=A^{\dagger}+W E_{A},
$$

where $W \in \mathbb{C}^{n \times m}$ is arbitrary.

(c) The following set inclusions hold

$$
\begin{array}{ll}
A^{\dagger} \in\left\{A^{(1,3,4)}\right\} \subseteq\left\{A^{(1,4)}\right\} \subseteq\left\{A^{(1)}\right\}, & A^{\dagger} \in\left\{A^{(1,3,4)}\right\} \subseteq\left\{A^{(1,3)}\right\} \subseteq\left\{A^{(1)}\right\}, \\
A^{\dagger} \in\left\{A^{(1,2,4)}\right\} \subseteq\left\{A^{(1,4)}\right\} \subseteq\left\{A^{(1)}\right\}, & A^{\dagger} \in\left\{A^{(1,2,4)}\right\} \subseteq\left\{A^{(1,2)}\right\} \subseteq\left\{A^{(1)}\right\}, \\
A^{\dagger} \in\left\{A^{(1,2,3)}\right\} \subseteq\left\{A^{(1,3)}\right\} \subseteq\left\{A^{(1)}\right\}, & A^{\dagger} \in\left\{A^{(1,2,3)}\right\} \subseteq\left\{A^{(1,2)}\right\} \subseteq\left\{A^{(1)}\right\},
\end{array}
$$

and following matrix set equalities hold

$$
\begin{array}{ll}
\left\{\left(A^{(1,3,4)}\right)^{*}\right\}=\left\{\left(A^{*}\right)^{(1,3,4)}\right\}, & \left\{\left(A^{(1,2,4)}\right)^{*}\right\}=\left\{\left(A^{*}\right)^{(1,2,3)}\right\}, \\
\left\{\left(A^{(1,2,3)}\right)^{*}\right\}=\left\{\left(A^{*}\right)^{(1,2,4)}\right\}, & \left\{\left(A^{(1,4)}\right)^{*}\right\}=\left\{\left(A^{*}\right)^{(1,3)}\right\}, \\
\left\{\left(A^{(1,3)}\right)^{*}\right\}=\left\{\left(A^{*}\right)^{(1,4)}\right\}, & \left\{\left(A^{(1,2)}\right)^{*}\right\}=\left\{\left(A^{*}\right)^{(1,2)}\right\}, \quad\left\{\left(A^{(1)}\right)^{*}\right\}=\left\{\left(A^{*}\right)^{(1)}\right\} .
\end{array}
$$

(d) [79] The following rank equalities hold

$$
\begin{aligned}
& \max _{A^{(1,3,4)}} r\left(A^{(1,3,4)}\right)=\min \{m, n\}, \\
& \max _{A^{(1,2,4)}} r\left(A^{(1,2,4)}\right)=r(A), \\
& \max _{A^{(1,2,3)}} r\left(A^{(1,2,3)}\right)=r(A), \\
& \max _{A^{(1,4)}} r\left(A^{(1,4)}\right)=\min \{m, n\}, \\
& \max _{A^{(1,3)}} r\left(A^{(1,3)}\right)=\min \{m, n\}, \\
& \max _{A^{(1,2)}} r\left(A^{(1,2)}\right)=r(A), \\
& \max _{A^{(1)}} r\left(A^{(1)}\right)=\min \{m, n\},
\end{aligned}
$$

$$
\begin{aligned}
\min _{A^{(1,3,4)}} r\left(A^{(1,3,4)}\right) & =r(A), \\
\min _{A^{(1,2,4)}} r\left(A^{(1,2,4)}\right) & =r(A), \\
\min _{A^{(1,2,3)}} r\left(A^{(1,2,3)}\right) & =r(A), \\
\min _{A^{(1,4)}} r\left(A^{(1,4)}\right) & =r(A), \\
\min _{A^{(1,3)}} r\left(A^{(1,3)}\right) & =r(A), \\
\min _{A^{(1,2)}} r\left(A^{(1,2)}\right) & =r(A), \\
\min _{A^{(1)}} r\left(A^{(1)}\right) & =r(A) .
\end{aligned}
$$

(e) The following matrix equalities hold

$$
\begin{aligned}
& A A^{(1,3,4)}=A A^{(1,2,3)}=A A^{(1,3)}=A A^{\dagger} \text { is unique, } \\
& A A^{(1,2,4)}=A A^{(1,4)}=A A^{(1,2)}=A A^{(1)}=A A^{\dagger}+A U E_{A}, \\
& A^{(1,3,4)} A=A^{(1,2,4)} A=A^{(1,4)} A=A^{\dagger} A \text { is unique, } \\
& A^{(1,2,3)} A=A^{(1,3)} A=A^{(1,2)} A=A^{(1)} A=A^{\dagger} A+F_{A} U A,
\end{aligned}
$$


where $U \in \mathbb{C}^{n \times m}$ is arbitrary. In particular,

$$
\begin{aligned}
& A A^{(1,2,4)}=A A^{(1,4)}=A A^{(1,2)}=A A^{(1)} \text { is unique } \Leftrightarrow \text { either } A=0 \text { or } r(A)=m, \\
& A^{(1,2,3)} A=A^{(1,3)} A=A^{(1,2)} A=A^{(1)} A \text { is unique } \Leftrightarrow \text { either } A=0 \text { or } r(A)=n .
\end{aligned}
$$

In addition, the following matrix equalities hold

$$
\begin{aligned}
& A^{(1)} A A^{(1)}=A^{(1)} A A^{(1,2)}=A^{(1)} A A^{(1,4)}=A^{(1)} A A^{(1,2,4)} \\
& =A^{(1,2)} A A^{(1)}=A^{(1,2)} A A^{(1,2)}=A^{(1,2)} A A^{(1,4)}=A^{(1,2)} A A^{(1,2,4)} \\
& =A^{(1,3)} A A^{(1)}=A^{(1,2)} A A^{(1,2)}=A^{(1,2)} A A^{(1,4)}=A^{(1,2)} A A^{(1,2,4)} \\
& =A^{(1,2,3)} A A^{(1)}=A^{(1,2,3)} A A^{(1,2)}=A^{(1,2,3)} A A^{(1,4)}=A^{(1,2,3)} A A^{(1,2,4)}=A^{(1,2)}, \\
& A^{(1)} A A^{(1,3)}=A^{(1)} A A^{(1,2,3)}=A^{(1)} A A^{(1,3,4)}=A^{(1)} A A^{\dagger} \\
& =A^{(1,2)} A A^{(1,3)}=A^{(1,2)} A A^{(1,2,3)}=A^{(1,2)} A A^{(1,3,4)}=A^{(1,2)} A A^{\dagger} \\
& =A^{(1,3)} A A^{(1,3)}=A^{(1,3)} A A^{(1,2,3)}=A^{(1,3)} A A^{(1,3,4)}=A^{(1,3)} A A^{\dagger} \\
& =A^{(1,2,3)} A A^{(1,3)}=A^{(1,2,3)} A A^{(1,2,3)}=A^{(1,2,3)} A A^{(1,3,4)}=A^{(1,2,3)} A A^{\dagger}=A^{(1,2,3)}, \\
& A^{(1,4)} A A^{(1)}=A^{(1,4)} A A^{(1,2)}=A^{(1,4)} A A^{(1,4)}=A^{(1,4)} A A^{(1,2,4)} \\
& =A^{(1,2,4)} A A^{(1)}=A^{(1,2,4)} A A^{(1,2)}=A^{(1,2,4)} A A^{(1,4)}=A^{(1,2,4)} A A^{(1,2,4)} \\
& =A^{(1,3,4)} A A^{(1)}=A^{(1,3,4)} A A^{(1,2)}=A^{(1,3,4)} A A^{(1,4)}=A^{(1,3,4)} A A^{(1,2,4)} \\
& =A^{\dagger} A A^{(1)}=A^{\dagger} A A^{(1,2)}=A^{\dagger} A A^{(1,4)}=A^{\dagger} A A^{(1,2,4)}=A^{(1,2,4)}, \\
& A^{(1,4)} A A^{(1,3)}=A^{(1,4)} A A^{(1,2,3)}=A^{(1,4)} A A^{(1,3,4)}=A^{(1,4)} A A^{\dagger} \\
& =A^{(1,2,4)} A A^{(1,3)}=A^{(1,2,4)} A A^{(1,2,3)}=A^{(1,2,4)} A A^{(1,3,4)}=A^{(1,2,4)} A A^{\dagger} \\
& =A^{(1,3,4)} A A^{(1,3)}=A^{(1,3,4)} A A^{(1,2,3)}=A^{(1,3,4)} A A^{(1,3,4)}=A^{(1,3,4)} A A^{\dagger} \\
& =A^{\dagger} A A^{(1,3)}=A^{\dagger} A A^{(1,2,3)}=A^{\dagger} A A^{(1,3,4)}=A^{\dagger} A A^{\dagger}=A^{\dagger} .
\end{aligned}
$$

(f) The following set inclusions

$$
\begin{aligned}
& P A^{\dagger} Q \in\left\{P A^{(1,3,4)} Q\right\} \subseteq\left\{P A^{(1,4)} Q\right\} \subseteq\left\{P A^{(1)} Q\right\}, \\
& P A^{\dagger} Q \in\left\{P A^{(1,3,4)} Q\right\} \subseteq\left\{P A^{(1,3)} Q\right\} \subseteq\left\{P A^{(1)} Q\right\}, \\
& P A^{\dagger} Q \in\left\{P A^{(1,2,4)} Q\right\} \subseteq\left\{P A^{(1,4)} Q\right\} \subseteq\left\{P A^{(1)} Q\right\}, \\
& P A^{\dagger} Q \in\left\{P A^{(1,2,4)} Q\right\} \subseteq\left\{P A^{(1,2)} Q\right\} \subseteq\left\{P A^{(1)} Q\right\}, \\
& P A^{\dagger} Q \in\left\{P A^{(1,2,3)} Q\right\} \subseteq\left\{P A^{(1,3)} Q\right\} \subseteq\left\{P A^{(1)} Q\right\}, \\
& P A^{\dagger} Q \in\left\{P A^{(1,2,3)} Q\right\} \subseteq\left\{P A^{(1,2)} Q\right\} \subseteq\left\{P A^{(1)} Q\right\}
\end{aligned}
$$

hold for any matrices $P$ and $Q$.

Lemma $3.2([78])$. Let $A \in \mathbb{C}^{m \times n}$ and $G \in \mathbb{C}^{n \times m}$. Then

$$
\begin{aligned}
\min _{A^{(1)}} r\left(A^{(1)}-G\right) & =r(A-A G A), \\
\min _{A^{(1,2)}} r\left(A^{(1,2)}-G\right) & =\max \{r(A-A G A), r(G)+r(A)-r(G A)-r(A G)\}, \\
\min _{A^{(1,3)}} r\left(A^{(1,3)}-G\right) & =r\left(A^{*} A G-A^{*}\right), \\
\min _{A^{(1,4)}} r\left(A^{(1,4)}-G\right) & =r\left(G A A^{*}-A^{*}\right), \\
\min _{A^{(1,2,3)}} r\left(A^{(1,2,3)}-G\right) & =r\left(A^{*} A G-A^{*}\right)+r\left[\begin{array}{c}
A^{*} \\
G
\end{array}\right]-r\left[\begin{array}{c}
A^{*} \\
A G
\end{array}\right], \\
\min _{A^{(1,2,4)}} r\left(A^{(1,2,4)}-G\right) & =r\left(G A A^{*}-A^{*}\right)+r\left[A^{*}, G\right]-r\left[A^{*}, G A\right], \\
\min _{A^{(1,3,4)}} r\left(A^{(1,3,4)}-G\right) & =r\left(A^{*} A G-A^{*}\right)+r\left(G A A^{*}-A^{*}\right)-r(A-A G A), \\
r\left(A^{\dagger}-G\right) & =r\left[\begin{array}{cc}
A^{*} A A^{*} & A^{*} \\
A^{*} & G
\end{array}\right]-r(A) .
\end{aligned}
$$


If $\mathscr{R}(G) \subseteq \mathscr{R}\left(A^{*}\right)$, then

$$
r\left(A^{\dagger}-G\right)=r\left(A^{*}-A^{*} A G\right)
$$

If $\mathscr{R}\left(G^{*}\right) \subseteq \mathscr{R}(A)$, then

$$
r\left(A^{\dagger}-G\right)=r\left(A^{*}-G A A^{*}\right) .
$$

If $\mathscr{R}(G) \subseteq \mathscr{R}\left(A^{*}\right)$ and $\mathscr{R}\left(G^{*}\right) \subseteq \mathscr{R}(A)$, then

$$
r\left(A^{\dagger}-G\right)=r(A-A G A) .
$$

In particular,

$$
\begin{aligned}
G \in\left\{A^{(1)}\right\} & \Leftrightarrow A G A=A, \\
G \in\left\{A^{(1,2)}\right\} & \Leftrightarrow A G A=A \text { and } r(G)=r(A), \\
G \in\left\{A^{(1,3)}\right\} & \Leftrightarrow A^{*} A G=A^{*}, \\
G \in\left\{A^{(1,4)}\right\} & \Leftrightarrow G A A^{*}=A^{*}, \\
G \in\left\{A^{(1,2,3)}\right\} & \Leftrightarrow A^{*} A G=A^{*} \text { and } r(G)=r(A) \Leftrightarrow A^{*} A G=A^{*} \text { and } G E_{A}=0, \\
G \in\left\{A^{(1,2,4)}\right\} & \Leftrightarrow G A A^{*}=A^{*} \text { and } r(G)=r(A) \Leftrightarrow G A A^{*}=A^{*} \text { and } F_{A} G=0, \\
G \in\left\{A^{(1,3,4)}\right\} & \Leftrightarrow A^{*} A G=A^{*} \text { and } G A A^{*}=A^{*}, \\
G=A^{\dagger} & \Leftrightarrow A^{*} A G=A^{*}, G A A^{*}=A^{*}, \text { and } r(G)=r(A) \\
& \Leftrightarrow A^{*} A G=A^{*}, G A A^{*}=A^{*}, G E_{A}=0, \text { and } F_{A} G=0 .
\end{aligned}
$$

Block matrix and rank of matrix are two fundamentals in linear algebra, but the block matrix method (BMM), the matrix rank method (MRM) are two fundamental and strong analytic methods that are widely used in matrix algebra and applications because they give one the ability to construct and analyze various complicated and nuanced matrix expressions and matrix equalities in a subtle and computationally tractable way. In view of the facts in Lemma 3.2, we first describe how to use the MRM in the study of set inclusions for generalized inverses of matrices.

Lemma 3.3. Let $A \in \mathbb{C}^{m \times n}$, and $f\left(A_{1}^{\left(i_{1}, \ldots, j_{1}\right)}, A_{2}^{\left(i_{2}, \ldots, j_{2}\right)}, \ldots, A_{k}^{\left(i_{k}, \ldots, j_{k}\right)}\right) \in \mathbb{C}^{n \times m}$ be a matrix expression composed by $A^{\left(i_{1}, \ldots, j_{1}\right)}, A_{2}^{\left(i_{2}, \ldots, j_{2}\right)}, \ldots, A_{k}^{\left(i_{k}, \ldots, j_{k}\right)}$. Then the following results hold.

(a) $\left\{A^{(1)}\right\} \supseteq \mathcal{D}_{f}$ if and only if

$$
\max _{A_{1}^{\left(i_{1}, \ldots, j_{1}\right)}, \ldots, A_{k}^{\left(i_{k}, \ldots, j_{k}\right)}} r(A-A f A)=0 .
$$

(b) $\left\{A^{(1,2)}\right\} \supseteq \mathcal{D}_{f}$ if and only if

$$
\max _{A_{1}^{\left(i_{1}, \ldots, j_{1}\right)}, \ldots, A_{k}^{\left(i_{k}, \ldots, j_{k}\right)}} r(A-A f A)=0 \text { and } \max _{A_{1}^{\left(i_{1}, \ldots, j_{1}\right)}, \ldots, A_{k}^{\left(i_{k}, \ldots, j_{k}\right)}} r(f)=r(A) .
$$

(c) $\left\{A^{(1,3)}\right\} \supseteq \mathcal{D}_{f}$ if and only if

$$
\max _{A_{1}^{\left(i_{1}, \ldots, j_{1}\right)}, \ldots, A_{k}^{\left(i_{k}, \ldots, j_{k}\right)}} r\left(A^{*}-A^{*} A f\right)=0 .
$$

(d) $\left\{A^{(1,4)}\right\} \supseteq \mathcal{D}_{f}$ if and only if

$$
\max _{A_{1}^{\left(i_{1}, \ldots, j_{1}\right)}, \ldots, A_{k}^{\left(i_{k}, \ldots, j_{k}\right)}} r\left(A^{*}-A f A A^{*}\right)=0
$$

(e) $\left\{A^{(1,2,3)}\right\} \supseteq \mathcal{D}_{f}$ if and only if

$$
\max _{A_{1}^{\left(i_{1}, \ldots, j_{1}\right)}, \ldots, A_{k}^{\left(i_{k}, \ldots, j_{k}\right)}} r\left(A^{*}-A^{*} A f A\right)=0 \text { and } \underset{A_{1}^{\left(i_{1}, \ldots, j_{1}\right)}, \ldots, A_{k}^{\left(i_{k}, \ldots, j_{k}\right)}}{\operatorname{man}}(f)=r(A) .
$$

(f) $\left\{A^{(1,2,4)}\right\} \supseteq \mathcal{D}_{f}$ if and only if

$$
\max _{A_{1}^{\left(i_{1}, \ldots, j_{1}\right)}, \ldots, A_{k}^{\left(i_{k}, \ldots, j_{k}\right)}} r\left(A^{*}-f A A^{*}\right)=0 \quad \text { and } \max _{A_{1}^{\left(i_{1}, \ldots, j_{1}\right)}, \ldots, A_{k}^{\left(i_{k}, \ldots, j_{k}\right)}} r(f)=r(A) .
$$


(g) $\left\{A^{(1,3,4)}\right\} \supseteq \mathcal{D}_{f} \Leftrightarrow\left\{A^{(1,3)}\right\} \supseteq \mathcal{D}_{f}$ and $\left\{A^{(1,4)}\right\} \supseteq \mathcal{D}_{f}$.

(h) $A^{\dagger}=\mathcal{D}_{f} \Leftrightarrow\left\{A^{(1,2,3)}\right\} \supseteq \mathcal{D}_{f}$ and $\left\{A^{(1,2,4)}\right\} \supseteq \mathcal{D}_{f} \Leftrightarrow f$ is invariant and $A^{\dagger}=f\left(A_{1}^{\dagger}, A_{2}^{\dagger}, \ldots, A_{k}^{\dagger}\right)$.

The assertions in Lemma 3.3 show strong requirements to establish various closed-formulas for calculating ranks of matrices and their generalized inverses, namely, if certain rank formulas associated with (1.8)-(1.10) are given, we can derive necessary and sufficient conditions for (1.8)-(1.10) to hold from the rank formulas, respectively.

Let $A \in \mathbb{C}^{m \times n}$ and $B \in \mathbb{C}^{n \times p}$. Applying (3.7)- 3.13) to $A B$, we obtain the following seven matrix expressions

$$
\begin{aligned}
& (A B)^{(1,3,4)}=(A B)^{\dagger}+F_{A B} V E_{A B}, \\
& (A B)^{(1,2,4)}=(A B)^{\dagger}+(A B)^{\dagger}(A B) V E_{A B}, \\
& (A B)^{(1,2,3)}=(A B)^{\dagger}+F_{A B} V(A B)(A B)^{\dagger}, \\
& (A B)^{(1,4)}=(A B)^{\dagger}+W E_{A B}, \\
& (A B)^{(1,3)}=(A B)^{\dagger}+F_{A B} V, \\
& (A B)^{(1,2)}=\left[(A B)^{\dagger}+F_{A B} V\right] A B\left[(A B)^{\dagger}+W E_{A B}\right], \\
& (A B)^{(1)}=(A B)^{\dagger}+F_{A B} V+W E_{A B},
\end{aligned}
$$

and

$$
\begin{aligned}
A B(A B)^{(1,3,4)} & =A B(A B)^{(1,2,3)}=A B(A B)^{(1,3)}=A B(A B)^{\dagger}, \\
A B(A B)^{(1,2,4)} & =A B(A B)^{(1,4)}=A B(A B)^{(1,2)}=A B(A B)^{(1)} \\
& =A B(A B)^{\dagger}+A B V E_{A B}, \\
(A B)^{(1,3,4)} A B & =(A B)^{(1,2,4)} A B=(A B)^{(1,4)} A B=(A B)^{\dagger} A B, \\
(A B)^{(1,2,3)} A B & =(A B)^{(1,3)} A B=(A B)^{(1,2)} A B=(A B)^{(1)} A B \\
& =(A B)^{\dagger} A B+F_{A B} V A B, \\
B(A B)^{(1,3,4)} A & =B(A B)^{\dagger} A+B F_{A B} V E_{A B} A, \\
B(A B)^{(1,2,4)} A & =B(A B)^{\dagger} A+B(A B)^{\dagger}(A B) V E_{A B} A, \\
B(A B)^{(1,2,3)} A & =B(A B)^{\dagger} A+B F_{A B} V(A B)(A B)^{\dagger} A, \\
B(A B)^{(1,4)} A & =B(A B)^{\dagger} A+B E_{A B} A, \\
B(A B)^{(1,3)} A & =B(A B)^{\dagger} A+B F_{A B} V A, \\
B(A B)^{(1,2)} A & =\left[B(A B)^{\dagger}+B F_{A B} V\right] A B\left[(A B)^{\dagger} A+W E_{A B} A\right], \\
B(A B)^{(1)} A & =B(A B)^{\dagger} A+B F_{A B} V B+A W E_{A B} A,
\end{aligned}
$$

where the two matrices $V$ and $W$ are arbitrary.

It is natural to see from the ROL in (1.7) that the starting point for the investigation of the ROL is to list out algebraic properties of the product $B^{\left(s_{2}, \ldots, t_{2}\right)} A^{\left(s_{1}, \ldots, t_{1}\right)}$ that are necessary to identify the ROL. On the other hand, a conventional technique in the study of matrix expressions that involve generalized inverses is representing the expressions in certain marginal matrix matrix-valued functions that involve variable matrices. Because generalized inverses of a matrix are linear or multilinear matrix-valued functions (MVF), we rewrite $B^{\left(s_{2}, \ldots, t_{2}\right)} A^{\left(s_{1}, \ldots, t_{1}\right)}$ as 63 linear or multilinear MVFs for the eight commonly-used types of generalized inverses of $A$ and $B$, respectively, except the unique case $B^{\dagger} A^{\dagger}$ for the Moore-Penrose inverses. For convenience of discussion, we present all the 63 MVFs in the following list

$$
\begin{aligned}
& B^{\dagger} A^{(1,3,4)}=B^{\dagger} A^{\dagger}+B^{\dagger} F_{A} U E_{A}, \\
& B^{\dagger} A^{(1,2,4)}=B^{\dagger} A^{\dagger}+B^{\dagger} A^{\dagger} A U E_{A}, \\
& B^{\dagger} A^{(1,2,3)}=B^{\dagger} A^{\dagger}+B^{\dagger} F_{A} U A A^{\dagger}, \\
& B^{\dagger} A^{(1,4)}=B^{\dagger} A^{\dagger}+B^{\dagger} U E_{A}, \\
& B^{\dagger} A^{(1,3)}=B^{\dagger} A^{\dagger}+B^{\dagger} F_{A} U, \\
& B^{\dagger} A^{(1,2)}=\left(B^{\dagger} A^{\dagger} A+B^{\dagger} F_{A} U_{1} A\right)\left(A^{\dagger}+A^{\dagger} A U_{2} E_{A}\right),
\end{aligned}
$$




$$
\begin{aligned}
& B^{\dagger} A^{(1)}=B^{\dagger} A^{\dagger}+B^{\dagger} F_{A} U_{1}+B^{\dagger} U_{2} E_{A}, \\
& B^{(1,3,4)} A^{\dagger}=B^{\dagger} A^{\dagger}+F_{B} V E_{B} A^{\dagger} \text {, } \\
& B^{(1,3,4)} A^{(1,3,4)}=\left(B^{\dagger}+F_{B} V E_{B}\right)\left(A^{\dagger}+F_{A} U E_{A}\right), \\
& B^{(1,3,4)} A^{(1,2,4)}=\left(B^{\dagger}+F_{B} V E_{B}\right)\left(A^{\dagger}+A^{\dagger} A U E_{A}\right), \\
& B^{(1,3,4)} A^{(1,2,3)}=\left(B^{\dagger}+F_{B} V E_{B}\right)\left(A^{\dagger}+F_{A} U A A^{\dagger}\right), \\
& B^{(1,3,4)} A^{(1,4)}=\left(B^{\dagger}+F_{B} V E_{B}\right)\left(A^{\dagger}+U E_{A}\right), \\
& B^{(1,3,4)} A^{(1,3)}=\left(B^{\dagger}+F_{B} V E_{B}\right)\left(A^{\dagger}+F_{A} U\right) \text {, } \\
& B^{(1,3,4)} A^{(1,2)}=\left(B^{\dagger}+F_{B} V E_{B}\right)\left(A^{\dagger} A+F_{A} U_{1} A\right)\left(A^{\dagger}+A^{\dagger} A U_{2} E_{A}\right), \\
& B^{(1,3,4)} A^{(1)}=\left(B^{\dagger}+F_{B} V E_{B}\right)\left(A^{\dagger}+F_{A} U_{1}+U_{2} E_{A}\right), \\
& B^{(1,2,4)} A^{\dagger}=B^{\dagger} A^{\dagger}+B^{\dagger} B V E_{B} A^{\dagger} \text {, } \\
& B^{(1,2,4)} A^{(1,3,4)}=\left(B^{\dagger}+B^{\dagger} B V E_{B}\right)\left(A^{\dagger}+F_{A} U E_{A}\right), \\
& B^{(1,2,4)} A^{(1,2,4)}=\left(B^{\dagger}+B^{\dagger} B V E_{B}\right)\left(A^{\dagger}+A^{\dagger} A U E_{A}\right), \\
& B^{(1,2,4)} A^{(1,2,3)}=\left(B^{\dagger}+B^{\dagger} B V E_{B}\right)\left(A^{\dagger}+F_{A} U A A^{\dagger}\right), \\
& B^{(1,2,4)} A^{(1,4)}=\left(B^{\dagger}+B^{\dagger} B V E_{B}\right)\left(A^{\dagger}+U E_{A}\right), \\
& B^{(1,2,4)} A^{(1,3)}=\left(B^{\dagger}+B^{\dagger} B V E_{B}\right)\left(A^{\dagger}+F_{A} U\right), \\
& B^{(1,2,4)} A^{(1,2)}=\left(B^{\dagger}+B^{\dagger} B V E_{B}\right)\left(A^{\dagger} A+F_{A} U_{1} A\right)\left(A^{\dagger}+A^{\dagger} A U_{2} E_{A}\right), \\
& B^{(1,2,4)} A^{(1)}=\left(B^{\dagger}+B^{\dagger} B V E_{B}\right)\left(A^{\dagger}+F_{A} U_{1}+U_{2} E_{A}\right), \\
& B^{(1,2,3)} A^{\dagger}=B^{\dagger} A^{\dagger}+F_{B} V B B^{\dagger} A^{\dagger}, \\
& B^{(1,2,3)} A^{(1,3,4)}=\left(B^{\dagger}+F_{B} V B B^{\dagger}\right)\left(A^{\dagger}+F_{A} U E_{A}\right), \\
& B^{(1,2,3)} A^{(1,2,4)}=\left(B^{\dagger}+F_{B} V B B^{\dagger}\right)\left(A^{\dagger}+A^{\dagger} A U E_{A}\right), \\
& B^{(1,2,3)} A^{(1,2,3)}=\left(B^{\dagger}+F_{B} V B B^{\dagger}\right)\left(A^{\dagger}+F_{A} U A A^{\dagger}\right), \\
& B^{(1,2,3)} A^{(1,4)}=\left(B^{\dagger}+F_{B} V B B^{\dagger}\right)\left(A^{\dagger}+U E_{A}\right), \\
& B^{(1,2,3)} A^{(1,3)}=\left(B^{\dagger}+F_{B} V B B^{\dagger}\right)\left(A^{\dagger}+F_{A} U\right), \\
& B^{(1,2,3)} A^{(1,2)}=\left(B^{\dagger}+F_{B} V B B^{\dagger}\right)\left(A^{\dagger} A+F_{A} U_{1} A\right)\left(A^{\dagger}+A^{\dagger} A U_{2} E_{A}\right), \\
& B^{(1,2,3)} A^{(1)}=\left(B^{\dagger}+F_{B} V B B^{\dagger}\right)\left(A^{\dagger}+F_{A} U_{1}+U_{2} E_{A}\right), \\
& B^{(1,4)} A^{\dagger}=B^{\dagger} A^{\dagger}+V E_{B} A^{\dagger} \text {, } \\
& B^{(1,4)} A^{(1,3,4)}=\left(B^{\dagger}+V E_{B}\right)\left(A^{\dagger}+F_{A} U E_{A}\right), \\
& B^{(1,4)} A^{(1,2,4)}=\left(B^{\dagger}+V E_{B}\right)\left(A^{\dagger}+A^{\dagger} A U E_{A}\right), \\
& B^{(1,4)} A^{(1,2,3)}=\left(B^{\dagger}+V E_{B}\right)\left(A^{\dagger}+F_{A} U A A^{\dagger}\right), \\
& B^{(1,4)} A^{(1,4)}=\left(B^{\dagger}+V E_{B}\right)\left(A^{\dagger}+U E_{A}\right), \\
& B^{(1,4)} A^{(1,3)}=\left(B^{\dagger}+V E_{B}\right)\left(A^{\dagger}+F_{A} U\right), \\
& B^{(1,4)} A^{(1,2)}=\left(B^{\dagger}+V E_{B}\right)\left(A^{\dagger} A+F_{A} U_{1} A\right)\left(A^{\dagger}+A^{\dagger} A U_{2} E_{A}\right), \\
& B^{(1,4)} A^{(1)}=\left(B^{\dagger}+V E_{B}\right)\left(A^{\dagger}+F_{A} U_{1}+U_{2} E_{A}\right), \\
& B^{(1,3)} A^{\dagger}=B^{\dagger} A^{\dagger}+F_{B} V A^{\dagger} \text {, } \\
& B^{(1,3)} A^{(1,3,4)}=\left(B^{\dagger}+F_{B} V\right)\left(A^{\dagger}+F_{A} U E_{A}\right), \\
& B^{(1,3)} A^{(1,2,4)}=\left(B^{\dagger}+F_{B} V\right)\left(A^{\dagger}+A^{\dagger} A U E_{A}\right), \\
& B^{(1,3)} A^{(1,2,3)}=\left(B^{\dagger}+F_{B} V\right)\left(A^{\dagger}+F_{A} U A A^{\dagger}\right), \\
& B^{(1,3)} A^{(1,4)}=\left(B^{\dagger}+F_{B} V\right)\left(A^{\dagger}+U E_{A}\right), \\
& B^{(1,3)} A^{(1,3)}=\left(B^{\dagger}+F_{B} V\right)\left(A^{\dagger}+F_{A} U\right), \\
& B^{(1,3)} A^{(1,2)}=\left(B^{\dagger}+F_{B} V\right)\left(A^{\dagger} A+F_{A} U_{1} A\right)\left(A^{\dagger}+A^{\dagger} A U_{2} E_{A}\right), \\
& B^{(1,3)} A^{(1)}=\left(B^{\dagger}+F_{B} V\right)\left(A^{\dagger}+F_{A} U_{1}+U_{2} E_{A}\right), \\
& B^{(1,2)} A^{\dagger}=\left(B^{\dagger}+F_{B} V_{1} B B^{\dagger}\right)\left(B B^{\dagger} A^{\dagger}+B V_{2} E_{B} A^{\dagger}\right) \text {, } \\
& B^{(1,2)} A^{(1,3,4)}=\left(B^{\dagger}+F_{B} V_{1} B B^{\dagger}\right)\left(B B^{\dagger}+B V_{2} E_{B}\right)\left(A^{\dagger}+F_{A} U E_{A}\right), \\
& B^{(1,2)} A^{(1,2,4)}=\left(B^{\dagger}+F_{B} V_{1} B B^{\dagger}\right)\left(B B^{\dagger}+B V_{2} E_{B}\right)\left(A^{\dagger}+A^{\dagger} A U E_{A}\right), \\
& B^{(1,2)} A^{(1,2,3)}=\left(B^{\dagger}+F_{B} V_{1} B B^{\dagger}\right)\left(B B^{\dagger}+B V_{2} E_{B}\right)\left(A^{\dagger}+F_{A} U A A^{\dagger}\right), \\
& B^{(1,2)} A^{(1,4)}=\left(B^{\dagger}+F_{B} V_{1} B B^{\dagger}\right)\left(B B^{\dagger}+B V_{2} E_{B}\right)\left(A^{\dagger}+U E_{A}\right) \text {, } \\
& B^{(1,2)} A^{(1,3)}=\left(B^{\dagger}+F_{B} V_{1} B B^{\dagger}\right)\left(B B^{\dagger}+B V_{2} E_{B}\right)\left(A^{\dagger}+F_{A} U\right),
\end{aligned}
$$




$$
\begin{aligned}
& B^{(1,2)} A^{(1,2)}=\left(B^{\dagger}+F_{B} V_{1} B B^{\dagger}\right)\left(B B^{\dagger}+B V_{2} E_{B}\right)\left(A^{\dagger} A+F_{A} U_{1} A\right)\left(A^{\dagger}+A^{\dagger} A U_{2} E_{A}\right), \\
& B^{(1,2)} A^{(1)}=\left(B^{\dagger}+F_{B} V_{1} B B^{\dagger}\right)\left(B B^{\dagger}+B V_{2} E_{B}\right)\left(A^{\dagger}+F_{A} U_{1}+U_{2} E_{A}\right), \\
& B^{(1)} A^{\dagger}=B^{\dagger} A^{\dagger}+F_{B} V_{1} A^{\dagger}+V_{2} E_{B} A^{\dagger}, \\
& B^{(1)} A^{(1,3,4)}=\left(B^{\dagger}+F_{B} V_{1}+V_{2} E_{B}\right)\left(A^{\dagger}+F_{A} U E_{A}\right), \\
& B^{(1)} A^{(1,2,4)}=\left(B^{\dagger}+F_{B} V_{1}+V_{2} E_{B}\right)\left(A^{\dagger}+A^{\dagger} A U E_{A}\right), \\
& B^{(1)} A^{(1,2,3)}=\left(B^{\dagger}+F_{B} V_{1}+V_{2} E_{B}\right)\left(A^{\dagger}+F_{A} U A A^{\dagger}\right), \\
& B^{(1)} A^{(1,4)}=\left(B^{\dagger}+F_{B} V_{1}+V_{2} E_{B}\right)\left(A^{\dagger}+U E_{A}\right), \\
& B^{(1)} A^{(1,3)}=\left(B^{\dagger}+F_{B} V_{1}+V_{2} E_{B}\right)\left(A^{\dagger}+F_{A} U\right), \\
& B^{(1)} A^{(1,2)}=\left(B^{\dagger}+F_{B} V_{1}+V_{2} E_{B}\right)\left(A^{\dagger} A+F_{A} U_{1} A\right)\left(A^{\dagger}+A^{\dagger} A U_{2} E_{A}\right), \\
& B^{(1)} A^{(1)}=\left(B^{\dagger}+F_{B} V_{1}+V_{2} E_{B}\right)\left(A^{\dagger}+F_{A} U_{1}+U_{2} E_{A}\right),
\end{aligned}
$$

where $V, V_{1}, V_{2}, U, U_{1}, U_{2}$ are arbitrary matrices of appropriate sizes. Correspondingly, the 64 products $M B^{\left(s_{2}, \ldots, t_{2}\right)} A^{(s, \ldots, t)} M$ for the eight commonly-used types of generalized inverses of $A$ and $B$ are divided into the following 4 groups:

$$
\begin{aligned}
& M B^{\dagger} A^{(1,3,4)} M=M B^{\dagger} A^{(1,2,4)} M=M B^{\dagger} A^{(1,4)} M=M B^{(1,3,4)} A^{\dagger} M \\
& =M B^{(1,3,4)} A^{(1,3,4)} M=M B^{(1,3,4)} A^{(1,2,4)} M=M B^{(1,3,4)} A^{(1,4)} M \\
& =M B^{(1,2,3)} A^{\dagger} M=M B^{(1,2,3)} A^{(1,3,4)} M=M B^{(1,2,3)} A^{(1,2,4)} M \\
& =M B^{(1,2,3)} A^{(1,4)} M=M B^{(1,3)} A^{\dagger} M=M B^{(1,3)} A^{(1,3,4)} M \\
& =M B^{(1,3)} A^{(1,2,4)} M=M B^{(1,3)} A^{(1,4)} M=M B^{\dagger} A^{\dagger} M \\
& M B^{\dagger} A^{(1,2,3)} M=M B^{\dagger} A^{(1,3)} M=M B^{\dagger} A^{(1,2)} M=M B^{\dagger} A^{(1)} M \\
& =M B^{(1,3,4)} A^{(1,2,3)} M=M B^{(1,3,4)} A^{(1,3)} M=M B^{(1,3,4)} A^{(1,2)} M \\
& =M B^{(1,3,4)} A^{(1)} M=M B^{(1,2,3)} A^{(1,2,3)} M=M B^{(1,2,3)} A^{(1,3)} M \\
& =M B^{(1,2,3)} A^{(1,2)} M=M B^{(1,2,3)} A^{(1)} M=M B^{(1,3)} A^{(1,2,3)} M \\
& =M B^{(1,3)} A^{(1,3)} M=M B^{(1,3)} A^{(1,2)} M=M B^{(1,3)} A^{(1)} M \\
& =M B^{\dagger} A^{\dagger} M+M B^{\dagger} F_{A} U M \\
& M B^{(1,2,4)} A^{\dagger} M=M B^{(1,2,4)} A^{(1,3,4)} M=M B^{(1,2,4)} A^{(1,2,4)} M \\
& =M B^{(1,2,4)} A^{(1,4)} M=M B^{(1,4)} A^{\dagger} M=M B^{(1,4)} A^{(1,3,4)} M \\
& =M B^{(1,4)} A^{(1,2,4)} M=M B^{(1,4)} A^{(1,4)} M=M B^{(1,2)} A^{\dagger} M \\
& =M B^{(1,2)} A^{(1,3,4)} M=M B^{(1,2)} A^{(1,2,4)} M=M B^{(1,2)} A^{(1,4)} M \\
& =M B^{(1)} A^{\dagger} M=M B^{(1)} A^{(1,3,4)} M=M B^{(1)} A^{(1,2,4)} M \\
& =M B^{(1)} A^{(1,4)} M=M B^{\dagger} A^{\dagger} M+M V E_{B} A^{\dagger} M, \\
& M B^{(1,2,4)} A^{(1,2,3)} M=M B^{(1,2,4)} A^{(1,3)} M=M B^{(1,2,4)} A^{(1,2)} M \\
& =M B^{(1,2,4)} A^{(1)} M=M B^{(1,4)} A^{(1,2,3)} M=M B^{(1,4)} A^{(1,3)} M \\
& =M B^{(1,4)} A^{(1,2)} M=M B^{(1,4)} A^{(1)} M=M B^{(1,2)} A^{(1,2,3)} M \\
& =M B^{(1,2)} A^{(1,3)} M=M B^{(1,2)} A^{(1,2)} M=M B^{(1,2)} A^{(1)} M \\
& =M B^{(1)} A^{(1,2,3)} M=M B^{(1)} A^{(1,3)} M=M B^{(1)} A^{(1,2)} M \\
& \left.=M B^{\dagger}+M V E_{B}\right)\left(A^{\dagger} M+F_{A} U M^{(1)} M\right. \\
& =
\end{aligned}
$$

where $V$ and $U$ are arbitrary matrices of appropriate sizes; the 64 products $M^{*} M B^{\left(s_{2}, \ldots, t_{2}\right)} A^{(s, \ldots, t)}$ for the eight commonly-used types of generalized inverses of $A$ and $B$ are divided into the following groups

$$
\begin{aligned}
M^{*} M B^{(1,3,4)} A^{\dagger} & =M^{*} M B^{(1,2,3)} A^{\dagger}=M^{*} M B^{(1,3)} A^{\dagger}=M^{*} M B^{\dagger} A^{\dagger}, \\
M^{*} M B^{\dagger} A^{(1,3,4)} & =M^{*} M B^{(1,3,4)} A^{(1,3,4)}=M^{*} M B^{(1,2,3)} A^{(1,3,4)} \\
& =M^{*} M B^{(1,3)} A^{(1,3,4)}=M^{*} M B^{\dagger} A^{\dagger}+M^{*} M B^{\dagger} F_{A} U E_{A},
\end{aligned}
$$




$$
\begin{aligned}
& M^{*} M B^{\dagger} A^{(1,2,4)}=M^{*} M B^{(1,3,4)} A^{(1,2,4)}=M^{*} M B^{(1,2,3)} A^{(1,2,4)} \\
& =M^{*} M B^{(1,3)} A^{(1,2,4)}=M^{*} M B^{\dagger} A^{\dagger}+M^{*} M B^{\dagger} A^{\dagger} A U E_{A}, \\
& M^{*} M B^{\dagger} A^{(1,2,3)}=M^{*} M B^{(1,3,4)} A^{(1,2,3)}=M^{*} M B^{(1,2,3)} A^{(1,2,3)} \\
& =M^{*} M B^{(1,3)} A^{(1,2,3)}=M^{*} M B^{\dagger} A^{\dagger}+M^{*} M B^{\dagger} F_{A} U A A^{\dagger}, \\
& M^{*} M B^{\dagger} A^{(1,4)}=M^{*} M B^{(1,3,4)} A^{(1,4)}=M^{*} M B^{(1,2,3)} A^{(1,4)} \\
& =M^{*} M B^{(1,3)} A^{(1,4)}=M^{*} M B^{\dagger} A^{\dagger}+M^{*} M B^{\dagger} U E_{A}, \\
& M^{*} M B^{\dagger} A^{(1,3)}=M^{*} M B^{(1,3,4)} A^{(1,3)}=M^{*} M B^{(1,2,3)} A^{(1,3)} \\
& =M^{*} M B^{(1,3)} A^{(1,3)}=M^{*} M B^{\dagger} A^{\dagger}+M^{*} M B^{\dagger} F_{A} U, \\
& M^{*} M B^{\dagger} A^{(1,2)}=M^{*} M B^{(1,3,4)} A^{(1,2)}=M^{*} M B^{(1,2,3)} A^{(1,2)} \\
& =M^{*} M B^{(1,3)} A^{(1,2)}=M^{*} M B^{\dagger}\left(A^{\dagger}+F_{A} U_{1}\right) A\left(A^{\dagger}+U_{2} E_{A}\right), \\
& M^{*} M B^{\dagger} A^{(1)}=M^{*} M B^{(1,3,4)} A^{(1)}=M^{*} M B^{(1,2,3)} A^{(1)} \\
& =M^{*} M B^{(1,3)} A^{(1)}=M^{*} M B^{\dagger}\left(A^{\dagger}+F_{A} U_{1}+U_{2} E_{A}\right) \\
& M^{*} M B^{(1,2,4)} A^{\dagger}=M^{*} M B^{(1,4)} A^{\dagger}=M^{*} M B^{(1,2)} A^{\dagger} \\
& =M^{*} M B^{(1)} A^{\dagger}=M^{*} M B^{\dagger} A^{\dagger}+M^{*} M V E_{B} A^{\dagger}, \\
& M^{*} M B^{(1,2,4)} A^{(1,3,4)}=M^{*} M B^{(1,4)} A^{(1,3,4)}=M^{*} M B^{(1,2)} A^{(1,3,4)} \\
& =M^{*} M B^{(1)} A^{(1,3,4)}=M^{*} M\left(B^{\dagger}+V E_{B}\right)\left(A^{\dagger}+F_{A} U E_{A}\right), \\
& M^{*} M B^{(1,2,4)} A^{(1,2,4)}=M^{*} M B^{(1,4)} A^{(1,2,4)}=M^{*} M B^{(1,2)} A^{(1,2,4)} \\
& =M^{*} M B^{(1)} A^{(1,2,4)}=M^{*} M\left(B^{\dagger}+V E_{B}\right)\left(A^{\dagger}+A^{\dagger} A U E_{A}\right), \\
& M^{*} M B^{(1,2,4)} A^{(1,2,3)}=M^{*} M B^{(1,4)} A^{(1,2,3)}=M^{*} M B^{(1,2)} A^{(1,2,3)} \\
& =M^{*} M B^{(1)} A^{(1,2,3)}=M^{*} M\left(B^{\dagger}+V E_{B}\right)\left(A^{\dagger}+F_{A} U A A^{\dagger}\right), \\
& M^{*} M B^{(1,2,4)} A^{(1,4)}=M^{*} M B^{(1,4)} A^{(1,4)}=M^{*} M B^{(1,2)} A^{(1,4)} \\
& =M^{*} M B^{(1)} A^{(1,4)}=M^{*} M\left(B^{\dagger}+V E_{B}\right)\left(A^{\dagger}+U E_{A}\right), \\
& M^{*} M B^{(1,2,4)} A^{(1,3)}=M^{*} M B^{(1,4)} A^{(1,3)}=M^{*} M B^{(1,2)} A^{(1,3)} \\
& =M^{*} M B^{(1)} A^{(1,3)}=M^{*} M\left(B^{\dagger}+V E_{B}\right)\left(A^{\dagger}+F_{A} U\right), \\
& M^{*} M B^{(1,2,4)} A^{(1,2)}=M^{*} M B^{(1,4)} A^{(1,2)}=M^{*} M B^{(1,2)} A^{(1,2)}=M^{*} M B^{(1)} A^{(1,2)} \\
& =M^{*} M\left(B^{\dagger}+V E_{B}\right)\left(A^{\dagger}+F_{A} U_{1}\right) A\left(A^{\dagger}+U_{2} E_{A}\right), \\
& M^{*} M B^{(1,2,4)} A^{(1)}=M^{*} M B^{(1,4)} A^{(1)}=M^{*} M B^{(1,2)} A^{(1)}=M^{*} M B^{(1)} A^{(1)} \\
& =M^{*} M\left(B^{\dagger}+V E_{B}\right)\left(A^{\dagger}+F_{A} U_{1}+U_{2} E_{A}\right),
\end{aligned}
$$

where $V, U, U_{1}$, and $U_{2}$ are arbitrary matrices of appropriate sizes; the 64 products $B^{\left(s_{2}, \ldots, t_{2}\right)} A^{(s, \ldots, t)} M M^{*}$ are classified as the following groups:

$$
\begin{aligned}
B^{\dagger} A^{(1,3,4)} M M^{*} & =B^{\dagger} A^{(1,2,4)} M M^{*}=B^{\dagger} A^{(1,4)} M M^{*}=B^{\dagger} A^{\dagger} M M^{*}, \\
B^{(1,3,4)} A^{\dagger} M M^{*} & =B^{(1,3,4)} A^{(1,3,4)} M M^{*}=B^{(1,3,4)} A^{(1,2,4)} M M^{*} \\
& =B^{(1,3,4)} A^{(1,4)} M M^{*}=B^{\dagger} A^{\dagger} M M^{*}+F_{B} V E_{B} A^{\dagger} M M^{*}, \\
B^{(1,2,4)} A^{\dagger} M M^{*} & =B^{(1,2,4)} A^{(1,3,4)} M M^{*}=B^{(1,2,4)} A^{(1,2,4)} M M^{*} \\
& =B^{(1,2,4)} A^{(1,4)} M M^{*}=B^{\dagger} A^{\dagger} M M^{*}+B^{\dagger} B V E_{B} A^{\dagger} M M^{*}, \\
B^{(1,2,3)} A^{\dagger} M M^{*} & =B^{(1,2,3)} A^{(1,3,4)} M M^{*}=B^{(1,2,3)} A^{(1,2,4)} M M^{*} \\
& =B^{(1,2,3)} A^{(1,4)} M M^{*}=B^{\dagger} A^{\dagger} M M^{*}+F_{B} V B B^{\dagger} A^{\dagger} M M^{*}, \\
B^{(1,4)} A^{(1,3,4)} M M^{*} & =B^{(1,4)} A^{(1,2,4)} M M^{*}=B^{(1,4)} A^{(1,4)} M M^{*} \\
& =B^{(1,4)} A^{\dagger} M M^{*}=B^{\dagger} A^{\dagger} M M^{*}+V E_{B} A^{\dagger} M M^{*}, \\
B^{(1,3)} A^{(1,3,4)} M M^{*} & =B^{(1,3)} A^{(1,2,4)} M M^{*}=B^{(1,3)} A^{(1,4)} M M^{*} \\
& =B^{(1,3)} A^{\dagger} M M^{*}=B^{\dagger} A^{\dagger} M M^{*}+F_{B} V A^{\dagger} M M^{*},
\end{aligned}
$$




$$
\begin{aligned}
B^{(1,2)} A^{(1,3,4)} M M^{*} & =B^{(1,2)} A^{(1,2,4)} M M^{*}=B^{(1,2)} A^{(1,4)} M M^{*}=B^{(1,2)} A^{\dagger} M M^{*} \\
& =\left(B^{\dagger}+F_{B} V_{1}\right) B\left(B^{\dagger}+V_{2} E_{B}\right) A^{\dagger} M M^{*}, \\
B^{(1)} A^{(1,3,4)} M M^{*} & =B^{(1)} A^{(1,2,4)} M M^{*}=B^{(1)} A^{(1,4)} M M^{*}=B^{(1)} A^{\dagger} M M^{*} \\
& =\left(B^{\dagger}+F_{B} V_{1}+V_{2} E_{B}\right) A^{\dagger} M M^{*}, \\
B^{\dagger} A^{(1,2,3)} M M^{*} & =B^{\dagger} A^{(1,3)} M M^{*}=B^{\dagger} A^{(1,2)} M M^{*}=B^{\dagger} A^{(1)} M M^{*} \\
& =B^{\dagger}\left(A^{\dagger}+F_{A} U\right) M M^{*}, \\
B^{(1,3,4)} A^{(1,2,3)} M M^{*} & =B^{(1,3,4)} A^{(1,3)} M M^{*}=B^{(1,3,4)} A^{(1,2)} M M^{*}=B^{(1,3,4)} A^{(1)} M M^{*} \\
& =\left(B^{\dagger}+F_{B} V E_{B}\right)\left(A^{\dagger}+F_{A} U\right) M M^{*}, \\
B^{(1,2,4)} A^{(1,2,3)} M M^{*} & =B^{(1,2,4)} A^{(1,3)} M M^{*}=B^{(1,2,4)} A^{(1,2)} M M^{*}=B^{(1,2,4)} A^{(1)} M M^{*} \\
& =\left(B^{\dagger}+B^{\dagger} B V E_{B}\right)\left(A^{\dagger}+F_{A} U\right) M M^{*}, \\
B^{(1,2,3)} A^{(1,2,3)} M M^{*} & =B^{(1,2,3)} A^{(1,3)} M M^{*}=B^{(1,2,3)} A^{(1,2)} M M^{*}=B^{(1,2,3)} A^{(1)} M M^{*} \\
& =\left(B^{\dagger}+F_{B} V B B^{\dagger}\right)\left(A^{\dagger}+F_{A} U\right) M M^{*}, \\
B^{(1,4)} A^{(1,2,3)} M M^{*} & =B^{(1,4)} A^{(1,3)} M M^{*}=B^{(1,4)} A^{(1,2)} M M^{*}=B^{(1,4)} A^{(1)} M M^{*} \\
& =\left(B^{\dagger}+V E_{B}\right)\left(A^{\dagger}+F_{A} U\right) M M^{*}, \\
B^{(1,3)} A^{(1,2,3)} M M^{*} & =B^{(1,3)} A^{(1,3)} M M^{*}=B^{(1,2,3)} A^{(1,2)} M M^{*}=B^{(1,3)} A^{(1)} M M^{*} \\
& =\left(B^{\dagger}+F_{B} V\right)\left(A^{\dagger}+F_{A} U\right) M M^{*}, \\
B^{(1,2)} A^{(1,2,3)} M M^{*} & =B^{(1,2)} A^{(1,3)} M M^{*}=B^{(1,2)} A^{(1,2)} M M^{*}=B^{(1,2)} A^{(1)} M M^{*} \\
& =\left(B^{\dagger}+F_{B} V_{1}\right) B\left(B^{\dagger}+V_{2} E_{B}\right)\left(A^{\dagger}+F_{A} U\right) M M^{*}, \\
B^{(1)} A^{(1,2,3)} M M^{*} & =B^{(1)} A^{(1,3)} M M^{*}=B^{(1)} A^{(1,2)} M M^{*}=B^{(1)} A^{(1)} M M^{*} \\
& =\left(B^{\dagger}+F_{B} V_{1}+V_{2} E_{B}\right)\left(A^{\dagger}+F_{A} U\right) M M^{*},
\end{aligned}
$$

where $U, V, V_{1}$, and $V_{2}$ are variable matrices of appropriate sizes. It is helpful to display all these matrix-valued functions together, while various fundamental algebraic characteristics of these products can be derived from the above matrix-valued functions, including the uniqueness and ranks of these matrix products, which we shall presented in Sections 6 and 8.

\section{Miscellaneous formulas for calculating ranks of matrices and their generalized inverses}

We next present a collection of known and new results concerning ranks of matrices and their generalized inverses to make the paper self-contained when establishing and simplifying various complicated matrix equalities.

Lemma 4.1. Let $P_{1} \in \mathbb{C}^{m \times p_{1}}, P_{2} \in \mathbb{C}^{m \times p_{2}}, Q_{1} \in \mathbb{C}^{m \times q_{1}}$, and $Q_{2} \in \mathbb{C}^{m \times q_{2}}$. Then

$$
\text { both } \mathscr{R}\left(P_{1}\right)=\mathscr{R}\left(Q_{1}\right) \text { and } \mathscr{R}\left(P_{2}\right)=\mathscr{R}\left(Q_{2}\right) \Rightarrow r\left[P_{1}, P_{2}\right]=r\left[Q_{1}, Q_{2}\right] \text {. }
$$

Lemma 4.2 ( [51]). Let $A \in \mathbb{C}^{m \times n}, B \in \mathbb{C}^{m \times k}, C \in \mathbb{C}^{l \times n}$, and $D \in \mathbb{C}^{l \times k}$. Then

$$
\begin{aligned}
r[A, B] & =r(A)+r\left(E_{A} B\right)=r(B)+r\left(E_{B} A\right), \\
r\left[\begin{array}{l}
A \\
C
\end{array}\right] & =r(A)+r\left(C F_{A}\right)=r(C)+r\left(A F_{C}\right), \\
r\left[\begin{array}{cc}
A & B \\
C & 0
\end{array}\right] & =r(B)+r(C)+r\left(E_{B} A F_{C}\right), \\
r\left[\begin{array}{cc}
A & B \\
C & D
\end{array}\right] & =r(A)+r\left[\begin{array}{cc}
0 & E_{A} B \\
C F_{A} & D-C A^{\dagger} B
\end{array}\right] .
\end{aligned}
$$

In particular, if $\mathscr{R}(B) \subseteq \mathscr{R}(A)$ and $\mathscr{R}\left(C^{*}\right) \subseteq \mathscr{R}\left(A^{*}\right)$, then

$$
r\left[\begin{array}{ll}
A & B \\
C & D
\end{array}\right]=r(A)+r\left(D-C A^{\dagger} B\right) .
$$

Furthermore, the following results hold. 
(a) $r[A, B]=r(A) \Leftrightarrow \mathscr{R}(B) \subseteq \mathscr{R}(A) \Leftrightarrow A A^{\dagger} B=B \Leftrightarrow E_{A} B=0$.

(b) $r\left[\begin{array}{l}A \\ C\end{array}\right]=r(A) \Leftrightarrow \mathscr{R}\left(C^{*}\right) \subseteq \mathscr{R}\left(A^{*}\right) \Leftrightarrow C A^{\dagger} A=C \Leftrightarrow C F_{A}=0$.

(c) $r[A, B]=r(A)+r(B) \Leftrightarrow \mathscr{R}(A) \cap \mathscr{R}(B)=\{0\} \Leftrightarrow \mathscr{R}\left[\left(E_{A} B\right)^{*}\right]=\mathscr{R}\left(B^{*}\right) \Leftrightarrow \mathscr{R}\left[\left(E_{B} A\right)^{*}\right]=\mathscr{R}\left(A^{*}\right)$.

(d) $r\left[\begin{array}{l}A \\ C\end{array}\right]=r(A)+r(C) \Leftrightarrow \mathscr{R}\left(A^{*}\right) \cap \mathscr{R}\left(C^{*}\right)=\{0\} \Leftrightarrow \mathscr{R}\left(C F_{A}\right)=\mathscr{R}(C) \Leftrightarrow \mathscr{R}\left(A F_{C}\right)=\mathscr{R}(A)$.

(e) $r\left[\begin{array}{ll}A & B \\ C & D\end{array}\right]=r(A) \Leftrightarrow \mathscr{R}(B) \subseteq \mathscr{R}(A), \mathscr{R}\left(C^{*}\right) \subseteq \mathscr{R}\left(A^{*}\right)$, and $C A^{\dagger} B=D$.

Lemma 4.3 ( $[89])$. Let $A \in \mathbb{C}^{m \times n}$ and $B \in \mathbb{C}^{m \times k}$, and denote $P_{A}=A A^{\dagger}$ and $P_{B}=B B^{\dagger}$. Then the following range equality

$$
\mathscr{R}(A) \cap \mathscr{R}(B)=\mathscr{R}\left(P_{A} P_{B}\right) \cap \mathscr{R}\left(P_{B} P_{A}\right)
$$

holds. Consequently, the following rank equalities

$$
\begin{aligned}
& r[A, B]=r(A)+r(B)-\operatorname{dim}[\mathscr{R}(A) \cap \mathscr{R}(B)]=r(A)+r(B)-\operatorname{dim}\left[\mathscr{R}\left(P_{A} P_{B}\right) \cap \mathscr{R}\left(P_{B} P_{A}\right)\right], \\
& r[A, B]=r(A)+r(B)-r\left(P_{A} P_{B}\right)-r\left(P_{B} P_{A}\right)+r\left[P_{A} P_{B}, P_{B} P_{A}\right]
\end{aligned}
$$

hold. In particular, the following results hold.

(a) $r[A, B]=r(A)+r(B) \Leftrightarrow r\left[P_{A} P_{B}, P_{B} P_{A}\right]=r\left(P_{A} P_{B}\right)+r\left(P_{B} P_{A}\right) \Leftrightarrow \mathscr{R}(A) \cap \mathscr{R}(B)=\{0\} \Leftrightarrow \mathscr{R}\left(P_{A} P_{B}\right) \cap$ $\mathscr{R}\left(P_{B} P_{A}\right)=\{0\}$.

(b) $r[A, B]=r(A)+r(B)-r\left(P_{A} P_{B}\right) \Leftrightarrow r\left[P_{A} P_{B}, P_{B} P_{A}\right]=r\left(P_{A} P_{B}\right)=r\left(P_{B} P_{A}\right) \Leftrightarrow \mathscr{R}\left(P_{A} P_{B}\right)=\mathscr{R}\left(P_{B} P_{A}\right) \Leftrightarrow$ $P_{A} P_{B}=P_{B} P_{A}$.

(c) $r[A, B]=r\left[P_{A} P_{B}, P_{B} P_{A}\right] \Leftrightarrow r\left(A^{*} B\right)=r(A)=r(B)$.

Lemma 4.4 ( $[72,73])$. Let $A \in \mathbb{C}^{m \times n}, B \in \mathbb{C}^{m \times k}, C \in \mathbb{C}^{l \times n}$, and $D \in \mathbb{C}^{l \times k}$. Then

$$
r\left(D-C A^{\dagger} B\right)=r\left[\begin{array}{cc}
A^{*} A A^{*} & A^{*} B \\
C A^{*} & D
\end{array}\right]-r(A) .
$$

In particular,

$$
\begin{aligned}
& r\left(D-C A A^{\dagger} B\right)=r\left[\begin{array}{cc}
A^{*} A & A^{*} B \\
C A & D
\end{array}\right]-r(A), \\
& r\left(D-C A^{\dagger} A B\right)=r\left[\begin{array}{cc}
A A^{*} & A B \\
C A^{*} & D
\end{array}\right]-r(A), \\
& r\left(A^{*}-A^{\dagger}\right)=r\left(A A^{*} A-A\right) .
\end{aligned}
$$

Lemma 4.5 ( [51]). Let $A \in \mathbb{C}^{m \times n}, B \in \mathbb{C}^{n \times p}$, and $C \in \mathbb{C}^{p \times q}$. Then

$$
r(A B)=r(A)+r(B)-n+r\left[\left(I_{n}-B B^{-}\right)\left(I_{n}-A^{-} A\right)\right]
$$

holds for all $A^{-}$and $B^{-}$. In particular, the following inequalities

$$
\max \{0, \quad r(A)+r(B)-n\} \leq r(A B) \leq \min \{r(A), \quad r(B)\}
$$

hold, and the following two groups of equivalent facts hold

$$
\begin{gathered}
r(A B)=r(A)+r(B)-n \Leftrightarrow\left(I_{n}-B B^{-}\right)\left(I_{n}-A^{-} A\right)=0, \\
r(A B)=n \Leftrightarrow r(A)=r(B)=n .
\end{gathered}
$$

The two formulas in following lemma are best known in elementary linear algebra.

Lemma 4.6. Let $A \in \mathbb{C}^{m \times m}$. Then

$$
\begin{aligned}
& r\left(A-A^{2}\right)=r\left(I_{m}-A\right)+r(A)-m, \\
& r\left(A-A^{3}\right)=r\left(I_{m}+A\right)+r\left(I_{m}-A\right)+r(A)-2 m .
\end{aligned}
$$


Lemma 4.7 ( 74$])$. Let $A \in \mathbb{C}^{m \times n}$ and assume that $X_{1}, X_{2} \in\left\{A^{(2)}\right\}$. Then

$$
r\left(X_{1}-X_{2}\right)=r\left[\begin{array}{c}
X_{1} \\
X_{2}
\end{array}\right]+r\left[X_{1}, X_{2}\right]-r\left(X_{1}\right)-r\left(X_{2}\right) .
$$

Lemma $4.8([89,100])$. Let $P, Q \in \mathbb{C}^{m \times m}$ be a pair of orthogonal projectors. Then

$$
\begin{aligned}
r(P+Q) & =r[P, Q], \\
r(P-Q) & =2 r[P, Q]-r(P)-r(Q), \\
r(P Q-Q P) & =r(P-Q)+r\left(P+Q-I_{m}\right)-m, \\
r(P Q-Q P) & =2 r[P, Q]+2 r(P Q)-2 r(P)-2 r(Q), \\
r(P Q-Q P) & =2 r[P Q, Q P]-2 r(P Q) .
\end{aligned}
$$

Lemma 4.9 ( $[100])$. Let $A \in \mathbb{C}^{m \times n}$ be given, and let $P \in \mathbb{C}^{m \times m}$ and $Q \in \mathbb{C}^{n \times n}$ be a pair of idempotent matrices.

$$
r(P A-A Q)=r\left[\begin{array}{c}
P A \\
Q
\end{array}\right]+r[A Q, P]-r(P)-r(Q) .
$$

Analytical formulas for calculating the maximum and minimum ranks of matrix expressions that involve variable matrices are highlights in the development of matrix calculus and applications over the past several decades. In the following, we present some basic formulas for calculating the maximum and minimum ranks of $A-B X C$.

Lemma 4.10 ( $[75,94])$. Let $A \in \mathbb{C}^{m \times n}, B \in \mathbb{C}^{m \times k}$, and $C \in \mathbb{C}^{l \times n}$. Then

$$
\begin{aligned}
& \max _{X \in \mathbb{C}^{k \times l}} r(A-B X C)=\min \left\{r[A, B], \quad r\left[\begin{array}{l}
A \\
C
\end{array}\right]\right\}, \\
& \min _{X \in \mathbb{C}^{k \times l}} r(A-B X C)=r[A, B]+r\left[\begin{array}{l}
A \\
C
\end{array}\right]-r\left[\begin{array}{ll}
A & B \\
C & 0
\end{array}\right] .
\end{aligned}
$$

Lemma 4.11. Let $A \in \mathbb{C}^{m \times n}$ and $B \in \mathbb{C}^{n \times p}$, and denote

$$
\begin{aligned}
& t_{1}=\min \{r(A)+r(B), \quad n\}, \quad t_{2}=r\left[A^{*}, B\right], \quad t_{3}=r\left[A^{*} A B, B\right], \\
& t_{4}=r\left[A A^{*} A B, A B\right], \quad t_{5}=r(A)+r\left[A^{*} A B, B\right]-r\left[A^{*}, B\right], \\
& t_{6}=r(A B), \quad t_{7}=r(A)+r(B)-r\left[A^{*}, B\right], \quad t_{8}=\max \{0, \quad r(A)+r(B)-n\} .
\end{aligned}
$$

Then the following inequalities hold

$$
t_{1} \geq t_{2} \geq t_{3} \geq t_{4} \geq t_{5} \geq t_{6} \geq t_{7} \geq t_{8}
$$

Proof. The first inequality in 4.29 follows from the two well-known inequalities $r\left[A^{*}, B\right] \leq r(A)+r(B)$ and $r\left[A^{*}, B\right] \leq n$. The second inequality in (4.29) follows directly the matrix product $\left[A^{*} A B, B\right]=\left[A^{*}, B\right]\left[\begin{array}{cc}A B & 0 \\ 0 & I_{p}\end{array}\right]$. The third inequality in (4.29) follows directly the matrix product $\left[A A^{*} A B, A B\right]=A\left[A^{*} A B, B\right]$. Furthermore, rewrite $\left[A A^{*} A B, A B\right]$ as a triple matrix product $\left[A A^{*} A B, A B\right]=A\left[A^{*} A, B\right]\left[\begin{array}{cc}B & 0 \\ 0 & I_{p}\end{array}\right]$, and applying the wellknown Frobenius' inequality $r(X Y Z) \geq r(X Y)+r(Y Z)-r(Y)$ to this triple product yields

$$
\begin{aligned}
r\left[A A^{*} A B, A B\right] & \geq r\left(A\left[A^{*} A, B\right]\right)+r\left(\left[A^{*} A, B\right]\left[\begin{array}{cc}
B & 0 \\
0 & I_{p}
\end{array}\right]\right)-r\left[A^{*} A, B\right] \\
& =r(A)+r\left[A^{*} A B, B\right]-r\left[A^{*}, B\right],
\end{aligned}
$$

establishing the fourth inequality in (4.29). Applying the Frobenius' inequality to $\left[A^{*} A B, B\right]=$ $\left[A^{*}, I_{n}\right]\left[\begin{array}{cc}A & 0 \\ 0 & B\end{array}\right]\left[\begin{array}{cc}B & 0 \\ 0 & I_{p}\end{array}\right]$ yields

$$
\begin{aligned}
r\left[A^{*} A B, B\right] & \geq r\left(\left[A^{*}, I_{n}\right]\left[\begin{array}{cc}
A & 0 \\
0 & B
\end{array}\right]\right)+r\left(\left[\begin{array}{cc}
A & 0 \\
0 & B
\end{array}\right]\left[\begin{array}{cc}
B & 0 \\
0 & I_{p}
\end{array}\right]\right)-r\left[\begin{array}{cc}
A & 0 \\
0 & B
\end{array}\right] \\
& =r\left[A^{*}, B\right]+r(A B)-r(A),
\end{aligned}
$$

establishing the fifth inequality in (4.29). Applying (4.2) and inequality $r(X-Y) \geq r(X)-r(Y)$ to $\left[A^{*}, B\right]$ yields

$$
r\left[A^{*}, B\right]=r\left(A^{*}\right)+r\left(B-A^{\dagger} A B\right) \geq r(A)+r(B)-r\left(A^{\dagger} A B\right)=r(A)+r(B)-r(A B),
$$

thus establishing the sixth inequality in (4.29). The last inequality in (4.29) is equivalent to the first inequality. 
Lemma 4.12. Let $A \in \mathbb{C}^{m \times n}, B \in \mathbb{C}^{n \times p}$, and denote

$$
\begin{aligned}
J & =\left[\begin{array}{cc}
A B(A B)^{*} A B & A B B^{*} B \\
A A^{*} A B & A B
\end{array}\right]=\left[\begin{array}{c}
A B B^{*} \\
A
\end{array}\right]\left[A^{*} A B, B\right], \\
s_{1} & =r\left[\begin{array}{c}
A B B^{*} \\
A
\end{array}\right]+r\left[A^{*} A B, B\right]-r\left[A^{*}, B\right], \\
s_{2} & =r\left[A^{*}, B\right]+2 r(A B)-r(A)-r(B), \\
s_{3} & =r\left[\begin{array}{c}
A B B^{*} B \\
A B
\end{array}\right]+r\left[A A^{*} A B, A B\right]-r(A B), \\
s_{4} & =r\left[\begin{array}{c}
A B B^{*} \\
A
\end{array}\right]+r\left[A^{*} A B, B\right]+r(A)+r(B)-2 r\left[A^{*}, B\right]-r(A B) .
\end{aligned}
$$

Then

$$
\begin{aligned}
& r(J) \geq s_{1} \geq s_{2} \geq r(A B), \\
& r(J) \geq s_{3} \geq s_{4} \geq r(A B) .
\end{aligned}
$$

Proof. Follows from Lemma 4.11

The fact in the following lemma is obvious.

Lemma 4.13. Let $\mathcal{S}$ and $\mathcal{T}$ be two matrix sets consisting of matrices of the same size, and let $P$ and $Q$ be two matrices of appropriate sizes. Then

$$
\mathcal{S} \supseteq \mathcal{T} \Rightarrow P \mathcal{S} Q \supseteq P \mathcal{T} Q
$$

Lemma 4.14 ( $[88])$. Let $\mathcal{M}$ and $\mathcal{N}$ be a pair of linear subspaces of $\mathbb{C}^{m}$, and let $P_{\mathcal{M}}$ and $P_{\mathcal{N}}$ be the orthogonal projectors onto $\mathcal{M}$ and $\mathcal{N}$, respectively. Then the following two dimension formulas

$$
\begin{aligned}
& \operatorname{dim}\left[(\mathcal{M}+\mathcal{N}) \cap\left(\mathcal{M}+\mathcal{N}^{\perp}\right) \cap\left(\mathcal{M}^{\perp}+\mathcal{N}\right) \cap\left(\mathcal{M}^{\perp}+\mathcal{N}^{\perp}\right)\right]=2 r\left(P_{\mathcal{M}} P_{\mathcal{N}}\right)-2 \operatorname{dim}(\mathcal{M} \cap \mathcal{N}), \\
& \operatorname{dim}\left[(\mathcal{M} \cap \mathcal{N}) \oplus\left(\mathcal{M} \cap \mathcal{N}^{\perp}\right) \oplus\left(\mathcal{M}^{\perp} \cap \mathcal{N}\right) \oplus\left(\mathcal{M}^{\perp} \cap \mathcal{N}^{\perp}\right)\right]=m-2 r\left(P_{\mathcal{M}} P_{\mathcal{N}}\right)+2 \operatorname{dim}(\mathcal{M} \cap \mathcal{N})
\end{aligned}
$$

hold. In particular, the following statements are equivalent:

(a) $(\mathcal{M}+\mathcal{N}) \cap\left(\mathcal{M}+\mathcal{N}^{\perp}\right) \cap\left(\mathcal{M}^{\perp}+\mathcal{N}\right) \cap\left(\mathcal{M}^{\perp}+\mathcal{N}^{\perp}\right)=\{0\}$.

(b) $(\mathcal{M} \cap \mathcal{N}) \oplus\left(\mathcal{M} \cap \mathcal{N}^{\perp}\right) \oplus\left(\mathcal{M}^{\perp} \cap \mathcal{N}\right) \oplus\left(\mathcal{M}^{\perp} \cap \mathcal{N}^{\perp}\right)=\mathbb{C}^{m}$.

(c) $r\left(P_{\mathcal{M}} P_{\mathcal{N}}\right)=\operatorname{dim}(\mathcal{M} \cap \mathcal{N})$ and/or $r\left(P_{\mathcal{N}} P_{\mathcal{M}}\right)=\operatorname{dim}(\mathcal{N} \cap \mathcal{M})$.

(d) $P_{\mathcal{M}} P_{\mathcal{N}}=P_{\mathcal{M} \cap \mathcal{N}}$ and $/$ or $P_{\mathcal{N}} P_{\mathcal{M}}=P_{\mathcal{N} \cap \mathcal{M}}$.

(e) $P_{\mathcal{M}} P_{\mathcal{N}}=P_{\mathcal{N}} P_{\mathcal{M}}$

\section{Characterizations of linear and multilinear matrix identities}

Matrix equations occupy a central place in the development of matrix calculus. Because generalized inverses of a matrix are defined to be solutions of some/all of the four Penrose equations, (1.7) can be regarded as a nonlinear matrix equation of the form $X=Y Z$ subject to the restrictions $X \in\left\{(A B)^{(i, \ldots, j)}\right\}, Y \in\left\{B^{\left(s_{2}, \ldots, t_{2}\right)}\right\}$, and $Z \in\left\{A^{\left(s_{1}, \ldots, t_{1}\right)}\right\}$. For a general matrix equation $f\left(X_{1}, \ldots, X_{k}\right)=0$, it is a fundamental and challenging problem to establish necessary and sufficient conditions for the equality to hold for all the variable matrices $X_{1}, \ldots, X_{k}$ due to the noncommutativity of matrix algebra. For the two simplest matrix equations $A X=0$ and $A X B=0$, it is old news that $A X=0$ holds for all $X$ if and only if $A=0 ; A X B=0$ holds for all $X$ if and only if either $A=0$ or $B=0$; see e.g., [7]. As matrix equations are given in general forms, the derivations and representations of identifying conditions become increasingly difficult for the matrix equations to always hold for all variable matrices in them. In a recent paper [41, Jiang and Tian recently have reconsidered the uniqueness (invariance property) of some general linear and multilinear matrix identities and have provided a short and readily comprehensible procedure of establishing various types of linear and multilinear matrix identities that involve separated variable matrices by means of the block matrix method (BMM). In this section, we present a group of known results concerning linear and multilinear matrix equations to hold for all the unknown matrices in them, which we shall use in the characterization of the invariance properties of products $B^{\left(s_{2}, \ldots, t_{2}\right)} A^{\left(s_{1}, \ldots, t_{1}\right)}$ with respect to the choices of $A^{\left(s_{1}, \ldots, t_{1}\right)}$ and $B^{\left(s_{2}, \ldots, t_{2}\right)}$. 
Lemma 5.1 ( 54$])$. Let

$$
B X=A
$$

be a given linear matrix equation, where $A \in \mathbb{C}^{m \times n}$ and $B \in \mathbb{C}^{m \times p}$ are known matrices, and $X \in \mathbb{C}^{p \times n}$ is an unknown matrix. Then

$$
\text { (5.1) is solvable for } X \Leftrightarrow \mathscr{R}(A) \subseteq \mathscr{R}(B) \Leftrightarrow B B^{\dagger} A=A \text {. }
$$

In this situation, the general solution of (5.1) can be written in the following parametric form

$$
X=B^{\dagger} A+F_{B} V
$$

where $V \in \mathbb{C}^{p \times n}$ is arbitrary. In particular, (5.1) holds for all matrices $X \in \mathbb{C}^{p \times n}$ if and only if both $A=0$ and $B=0$, or equivalently, $[A, B]=0$.

Lemma $5.2([54])$. Let

$$
B X C=A
$$

be a given linear matrix equation, where $A \in \mathbb{C}^{m \times n}, B \in \mathbb{C}^{m \times p}$, and $C \in \mathbb{C}^{q \times n}$ are known matrices. Then the following statements are equivalent:

(a) Eq. (5.4) is solvable for $X \in \mathbb{C}^{p \times q}$.

(b) Both $\mathscr{R}(A) \subseteq \mathscr{R}(B)$ and $\mathscr{R}\left(A^{*}\right) \subseteq \mathscr{R}\left(C^{*}\right)$.

(c) $r[A, B]=r(B)$ and $r\left[\begin{array}{l}A \\ C\end{array}\right]=r(C)$.

(d) $B B^{\dagger} A=A$ and $A C^{\dagger} C=A$.

(e) $B B^{\dagger} A C^{\dagger} C=A$.

In this situation, the general solution of (5.4) can be written in the following parametric form

$$
X=A^{\dagger} C B^{\dagger}+F_{A} V+W E_{B}
$$

where $V, W \in \mathbb{C}^{p \times q}$ are arbitrary. In particular, (5.4) holds for all matrices $X \in \mathbb{C}^{p \times q}$ if and only if

$$
\text { either }[A, B]=0 \text { or }\left[\begin{array}{l}
A \\
C
\end{array}\right]=0 \text {. }
$$

Lemma $5.3([41])$. Let

$$
B_{1} X_{1} C_{1}+B_{2} X_{2} C_{2}=A
$$

be a given linear matrix equation, where $A \in \mathbb{C}^{m \times n}, B_{1} \in \mathbb{C}^{m \times p_{1}}, B_{2} \in \mathbb{C}^{m \times p_{2}}, C_{1} \in \mathbb{C}^{q_{1} \times n}$, and $C_{2} \in \mathbb{C}^{q_{2} \times n}$ are known matrices. Then the following results hold.

(a) Eq. 5.7) holds for all matrices $X_{1} \in \mathbb{C}^{p_{1} \times q_{1}}$ and $X_{2} \in \mathbb{C}^{p_{2} \times q_{2}}$ if and only if the 5 given matrices satisfy one of the following 4 block matrix equalities:

$$
\text { (i) }\left[A, B_{1}, B_{2}\right]=0 . \quad \text { (ii) }\left[\begin{array}{cc}
A & B_{1} \\
C_{2} & 0
\end{array}\right]=0 . \quad \text { (iii) }\left[\begin{array}{cc}
A & B_{2} \\
C_{1} & 0
\end{array}\right]=0 . \quad \text { (iv) }\left[\begin{array}{c}
A \\
C_{1} \\
C_{2}
\end{array}\right]=0 \text {. }
$$

(b) Under the assumptions that $\mathscr{R}\left(B_{1}\right) \subseteq \mathscr{R}\left(B_{2}\right)$ and $\mathscr{R}\left(C_{1}^{*}\right) \supseteq \mathscr{R}\left(C_{2}^{*}\right)$, (5.7) holds for all matrices $X_{1}$ and $X_{2}$ if and only if one of the following 2 block matrix equalities holds:

$$
\text { (i) }\left[A, B_{2}\right]=0 . \quad \text { (ii) }\left[\begin{array}{c}
A \\
C_{1}
\end{array}\right]=0 . \quad \text { (iii) }\left[\begin{array}{cc}
A & B_{1} \\
C_{2} & 0
\end{array}\right]=0
$$

Several multilinear versions of matrix identities were also established in [41] as follows.

Lemma 5.4. Let

$$
\left(A_{1}+B_{1} X_{1} C_{1}\right)\left(A_{2}+B_{2} X_{2} C_{2}\right)=A
$$

be a given multilinear matrix equation, where $A \in \mathbb{C}^{m \times n}, A_{1} \in \mathbb{C}^{m \times s}, B_{1} \in \mathbb{C}^{m \times p_{1}}, C_{1} \in \mathbb{C}^{q_{1} \times s}, A_{2} \in \mathbb{C}^{s \times n}$, $B_{2} \in \mathbb{C}^{s \times p_{2}}$, and $C_{2} \in \mathbb{C}^{q_{2} \times n}$ are known matrices. Then the following results hold. 
(a) Eq. 5.8 holds for all matrices $X_{1} \in \mathbb{C}^{p_{1} \times q_{1}}$ and $X_{2} \in \mathbb{C}^{p_{2} \times q_{2}}$ if and only if one of the following 4 block matrix equalities holds:
(i) $\left[A_{1} A_{2}-A, A_{1} B_{2}, B_{1}\right]=0$.
(ii) $\left[\begin{array}{cc}A_{1} A_{2}-A & B_{1} \\ C_{2} & 0\end{array}\right]=0$.
(iii) $\left[\begin{array}{cc}A_{1} A_{2}-A & A_{1} B_{2} \\ C_{1} A_{2} & C_{1} B_{2}\end{array}\right]=0$.
(iv) $\left[\begin{array}{c}A_{1} A_{2}-A \\ C_{1} A_{2} \\ C_{2}\end{array}\right]=0$.

(b) Under the assumption $\mathscr{R}\left(A_{1}\right) \subseteq \mathscr{R}\left(B_{1}\right)$ and $\mathscr{R}\left(A_{2}\right) \subseteq \mathscr{R}\left(B_{2}\right),(5.8)$ holds for all $X_{1} \in \mathbb{C}^{p_{1} \times q_{1}}$ and $X_{2} \in \mathbb{C}^{p_{2} \times q_{2}}$ if and only if one of the following 3 block matrix equalities holds:
(i) $\left[A, B_{1}\right]=0$
(ii) $\left[\begin{array}{cc}A & A_{1} B_{2} \\ 0 & C_{1} B_{2}\end{array}\right]=0$.
(iii) $\left[\begin{array}{c}A_{1} A_{2}-A \\ C_{1} A_{2} \\ C_{2}\end{array}\right]=0$.

(c) Under the assumption $\mathscr{R}\left(A_{1}^{*}\right) \subseteq \mathscr{R}\left(C_{1}^{*}\right)$ and $\mathscr{R}\left(A_{2}^{*}\right) \subseteq \mathscr{R}\left(C_{2}^{*}\right)$, (5.8) holds for all $X_{1} \in \mathbb{C}^{p_{1} \times q_{1}}$ and $X_{2} \in \mathbb{C}^{p_{2} \times q_{2}}$ if and only if one of the following 3 block matrix equalities holds:
(i) $\left[A_{1} A_{2}-A, A_{1} B_{2}, B_{1}\right]=0$.
(ii) $\left[\begin{array}{cc}A & 0 \\ C_{1} A_{2} & C_{1} B_{2}\end{array}\right]=0$.
(iii) $\left[\begin{array}{c}A \\ C_{2}\end{array}\right]=0$

(d) Under the assumption $\mathscr{R}\left(A_{1}\right) \subseteq \mathscr{R}\left(B_{1}\right)$ and $\mathscr{R}\left(A_{2}^{*}\right) \subseteq \mathscr{R}\left(C_{2}^{*}\right)$, (5.8) holds for all $X_{1} \in \mathbb{C}^{p_{1} \times q_{1}}$ and $X_{2} \in \mathbb{C}^{p_{2} \times q_{2}}$ if and only if one of the following 3 block matrix equalities holds:
(i) $\left[A, B_{1}\right]=0$.
(ii) $\left[\begin{array}{c}A \\ C_{2}\end{array}\right]=0$.
(iii) $\left[\begin{array}{cc}A_{1} A_{2}-A & A_{1} B_{2} \\ C_{1} A_{2} & C_{1} B_{2}\end{array}\right]=0$.

(e) Under the assumption $\mathscr{R}\left(A_{1}^{*}\right) \subseteq \mathscr{R}\left(C_{1}^{*}\right)$ and $\mathscr{R}\left(A_{2}\right) \subseteq \mathscr{R}\left(B_{2}\right),(5.8)$ holds for all $X_{1} \in \mathbb{C}^{p_{1} \times q_{1}}$ and $X_{2} \in \mathbb{C}^{p_{2} \times q_{2}}$ if and only if one of the following 4 block matrix equalities holds:
(i) $\left[A, A_{1} B_{2}, B_{1}\right]=0$.
(ii) $\left[\begin{array}{cc}A_{1} A_{2}-A & B_{1} \\ C_{2} & 0\end{array}\right]=0$.
(iv) $\left[\begin{array}{c}A \\ C_{1} A_{2} \\ C_{2}\end{array}\right]=0$.

Lemma 5.5. Let

$$
\left(A_{1}+B_{1} X_{1} C_{1}\right)\left(A_{2}+B_{2} X_{2} C_{2}\right)\left(A_{3}+B_{3} X_{3} C_{3}\right)=A
$$

be a given multilinear matrix equation, where $A, A_{i}, B_{i}$, and $C_{i}$ are known matrices of appropriate sizes, $i=1,2,3$. Then the following results hold.

(a) Eq. 5.9 holds for all matrices $X_{1}, X_{2}$, and $X_{3}$ if and only if one of the following 8 block matrix equalities holds:
(i) $\left[A_{1} A_{2} A_{3}-A, A_{1} A_{2} B_{3}, A_{1} B_{2}, B_{1}\right]=0$.
(iii) $\left[\begin{array}{ccc}A_{1} A_{2} A_{3}-A & A_{1} A_{2} B_{3} & B_{1} \\ C_{2} A_{3} & C_{2} B_{3} & 0\end{array}\right]=$
(vii) $\left[\begin{array}{c}A_{1} A_{2} A_{3}-A \\ C_{2} A_{3} \\ C_{3}\end{array}\right.$

$$
\left.\begin{array}{c}
B_{1} \\
0 \\
0
\end{array}\right]=0
$$

(ii)

(iv) 
(b) Under the assumption $\mathscr{R}\left(A_{1}\right) \subseteq \mathscr{R}\left(B_{1}\right)$ and $\mathscr{R}\left(A_{3}\right) \subseteq \mathscr{R}\left(B_{3}\right)$, (5.9) holds for all matrices $X_{1}, X_{2}$, and $X_{3}$ if and only if one of the following 5 block matrix equalities holds

$$
\begin{aligned}
& \text { (i) }\left[A, B_{1}\right]=0 . \quad \text { (ii) }\left[\begin{array}{ccc}
A & A_{1} A_{2} B_{3} & A_{1} B_{2} \\
0 & C_{1} A_{2} B_{3} & C_{1} B_{2}
\end{array}\right]=0 . \quad \text { (iii) }\left[\begin{array}{cc}
A & A_{1} A_{2} B_{3} \\
0 & C_{1} A_{2} B_{3} \\
0 & C_{2} B_{3}
\end{array}\right]=0 . \\
& \text { (iv) }\left[\begin{array}{cc}
A_{1} A_{2} A_{3}-A & A_{1} B_{2} \\
C_{1} A_{2} A_{3} & C_{1} B_{2} \\
C_{3} & 0
\end{array}\right]=0 . \quad(\mathrm{v})\left[\begin{array}{c}
A_{1} A_{2} A_{3}-A \\
C_{1} A_{2} A_{3} \\
C_{2} A_{3} \\
C_{3}
\end{array}\right]=0 .
\end{aligned}
$$

(c) Under the assumption $\mathscr{R}\left(A_{1}^{*}\right) \subseteq \mathscr{R}\left(C_{1}^{*}\right)$ and $\mathscr{R}\left(A_{3}^{*}\right) \subseteq \mathscr{R}\left(C_{3}^{*}\right)$, (5.9) holds for all matrices $X_{1}, X_{2}$, and $X_{3}$ if and only if one of the following 5 block matrix equalities holds

$$
\begin{aligned}
& \text { (i) }\left[A_{1} A_{2} A_{3}-A, A_{1} A_{2} B_{3}, A_{1} B_{2}, B_{1}\right]=0 \text {. } \\
& \text { (ii) }\left[\begin{array}{ccc}
A & 0 & 0 \\
C_{1} A_{2} A_{3} & C_{1} A_{2} B_{3} & C_{1} B_{2}
\end{array}\right]=0 . \quad \text { (iii) } \quad\left[\begin{array}{ccc}
A_{1} A_{2} A_{3}-A & A_{1} A_{2} B_{3} & B_{1} \\
C_{2} A_{3} & C_{2} B_{3} & 0
\end{array}\right]=0 \text {. } \\
& \text { (iv) }\left[\begin{array}{cc}
A & 0 \\
C_{1} A_{2} A_{3} & C_{1} A_{2} B_{3} \\
C_{2} A_{3} & C_{2} B_{3}
\end{array}\right]=0 . \quad \text { (v) }\left[\begin{array}{c}
A \\
C_{3}
\end{array}\right]=0 \text {. }
\end{aligned}
$$

(d) Under the assumption $\mathscr{R}\left(A_{1}\right) \subseteq \mathscr{R}\left(B_{1}\right)$ and $\mathscr{R}\left(A_{3}^{*}\right) \subseteq \mathscr{R}\left(C_{3}^{*}\right)$, (5.9) holds for all matrices $X_{1}, X_{2}$, and $X_{3}$ if and only if one of the following 4 block matrix equalities holds

$$
\begin{array}{cc}
\text { (i) }\left[A, B_{1}\right]=0 . & \text { (ii) }\left[\begin{array}{c}
A \\
C_{3}
\end{array}\right]=0 . \\
\text { (iii) }\left[\begin{array}{ccc}
A_{1} A_{2} A_{3}-A & A_{1} A_{2} B_{3} & A_{1} B_{2} \\
C_{1} A_{2} A_{3} & C_{1} A_{2} B_{3} & C_{1} B_{2}
\end{array}\right]=0 . \quad \text { (vi) }\left[\begin{array}{cc}
A_{1} A_{2} A_{3}-A & A_{1} A_{2} B_{3} \\
C_{1} A_{2} A_{3} & C_{1} A_{2} B_{3} \\
C_{2} A_{3} & C_{2} B_{3}
\end{array}\right]=0 .
\end{array}
$$

(e) Under the assumption $\mathscr{R}\left(A_{1}^{*}\right) \subseteq \mathscr{R}\left(C_{1}^{*}\right)$ and $\mathscr{R}\left(A_{3}\right) \subseteq \mathscr{R}\left(B_{3}\right),(5.9)$ holds fo all matrices $X_{1}, X_{2}$, and $X_{3}$ if and only if one of the following 8 block matrix equalities holds

$$
\begin{aligned}
& \text { (i) }\left[A, A_{1} A_{2} B_{3}, A_{1} B_{2}, B_{1}\right]=0 \text {. } \\
& \text { (ii) }\left[\begin{array}{ccc}
A & 0 & 0 \\
0 & C_{1} A_{2} B_{3} & C_{1} B_{2}
\end{array}\right]=0 \text {. } \\
& \text { (iii) }\left[\begin{array}{ccc}
A & A_{1} A_{2} B_{3} & B_{1} \\
0 & C_{2} B_{3} & 0
\end{array}\right]=0 \text {. } \\
& \text { (v) }\left[\begin{array}{cc}
A & 0 \\
0 & C_{1} A_{2} B_{3} \\
0 & C_{2} B_{3}
\end{array}\right]=0 \text {. } \\
& \text { (vii) }\left[\begin{array}{cc}
A_{1} A_{2} A_{3}-A & B_{1} \\
C_{2} A_{3} & 0 \\
C_{3} & 0
\end{array}\right]=0 \text {. }
\end{aligned}
$$

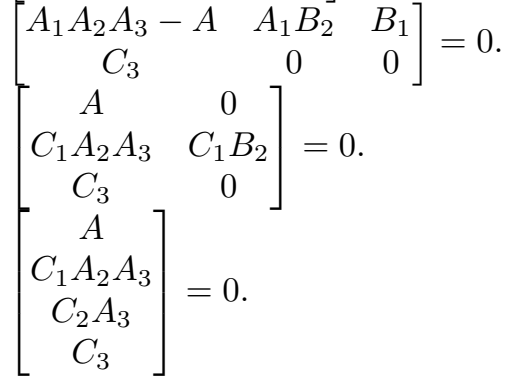

Lemma 5.6. The matrix equation

$$
\left(A_{1}+B_{1} X_{1} C_{1}\right)\left(A_{2}+B_{2} X_{2}+Y_{2} C_{2}\right)\left(A_{3}+B_{3} X_{3} C_{3}\right)=A
$$

holds for all matrices $X_{1}, X_{2}, X_{3}$, and $Y_{2}$ if and only if one of the following 8 block matrix equalities holds:
(i) $\left[A, A_{1}, B_{1}\right]=0$.
(iii) $\left[\begin{array}{cc}A & 0 \\ A_{3} & B_{3}\end{array}\right]=0$.
(ii) $\left[\begin{array}{cc}A & A_{1} \\ 0 & C_{1}\end{array}\right]=0$.
(iv) $\left[\begin{array}{c}A \\ A_{3} \\ C_{3}\end{array}\right]=0$.
(v) $\left[\begin{array}{cccc}A_{1} A_{2} A_{3}-A & A_{1} A_{2} B_{3} & A_{1} B_{2} & B_{1} \\ C_{2} A_{3} & C_{2} B_{3} & 0 & 0\end{array}\right]=0$. $A_{1} A_{2} A_{3}-A$ $\left.\begin{array}{ccc}C_{3} & 0 & 0 \\ C_{1} & 0 & 0\end{array}\right]=0$
(vii) $\left[\begin{array}{ccc}A_{1} A_{2} A_{3}-A & A_{1} A_{2} B_{3} & A_{1} B_{2} \\ C_{1} A_{2} A_{3} & C_{1} A_{2} B_{3} & C_{1} B_{2} \\ C_{2} A_{3} & C_{2} B_{3} & 0\end{array}\right]=0$.

$$
\left[\begin{array}{cc}
A_{1} A_{2} A_{3}-A & A_{1} B_{2} \\
C_{1} A_{2} A_{3} & C_{1} B_{2} \\
C_{2} A_{3} & 0 \\
C_{3} & 0
\end{array}\right]=0
$$


Under the assumption $\mathscr{R}\left(A_{1}\right) \subseteq \mathscr{R}\left(B_{1}\right)$ and $\mathscr{R}\left(A_{3}^{*}\right) \subseteq \mathscr{R}\left(C_{3}^{*}\right)$, 5.10 holds for all matrices $X_{1}, X_{2}, X_{3}$, and $Y_{2}$ if and only if one of the following 5 block matrix equalities holds:

$$
\begin{aligned}
& \text { (i) }\left[A, B_{1}\right]=0 . \quad \text { (ii) }\left[\begin{array}{c}
A \\
C_{3}
\end{array}\right]=0 . \quad \text { (iii) }\left[\begin{array}{cc}
A & A_{1} \\
0 & C_{1}
\end{array}\right]=0 . \quad \text { (iv) }\left[\begin{array}{cc}
A & 0 \\
A_{3} & B_{3}
\end{array}\right]=0 \text {. } \\
& \text { (v) }\left[\begin{array}{ccc}
A_{1} A_{2} A_{3}-A & A_{1} A_{2} B_{3} & A_{1} B_{2} \\
C_{1} A_{2} A_{3} & C_{1} A_{2} B_{3} & C_{1} B_{2} \\
C_{2} A_{3} & C_{2} B_{3} & 0
\end{array}\right]=0 .
\end{aligned}
$$

Lemma 5.7. Let

$$
\left(A_{1}+B_{1} X_{1} C_{1}+D_{1} Y_{1} E_{1}\right)\left(A_{2}+B_{2} X_{2} C_{2}+D_{2} Y_{2} E_{2}\right)=A
$$

be a given multilinear matrix equation, where $A, A_{i}, B_{i}, C_{i}, D_{i}$, and $E_{i}$ are known matrices of appropriate sizes, $i=1,2$. Then the following results hold.

(a) Eq. 5.11) holds for all matrices $X_{1}, X_{2}, Y_{1}$, and $Y_{2}$ if and only if one of the following 16 block matrix equalities holds:

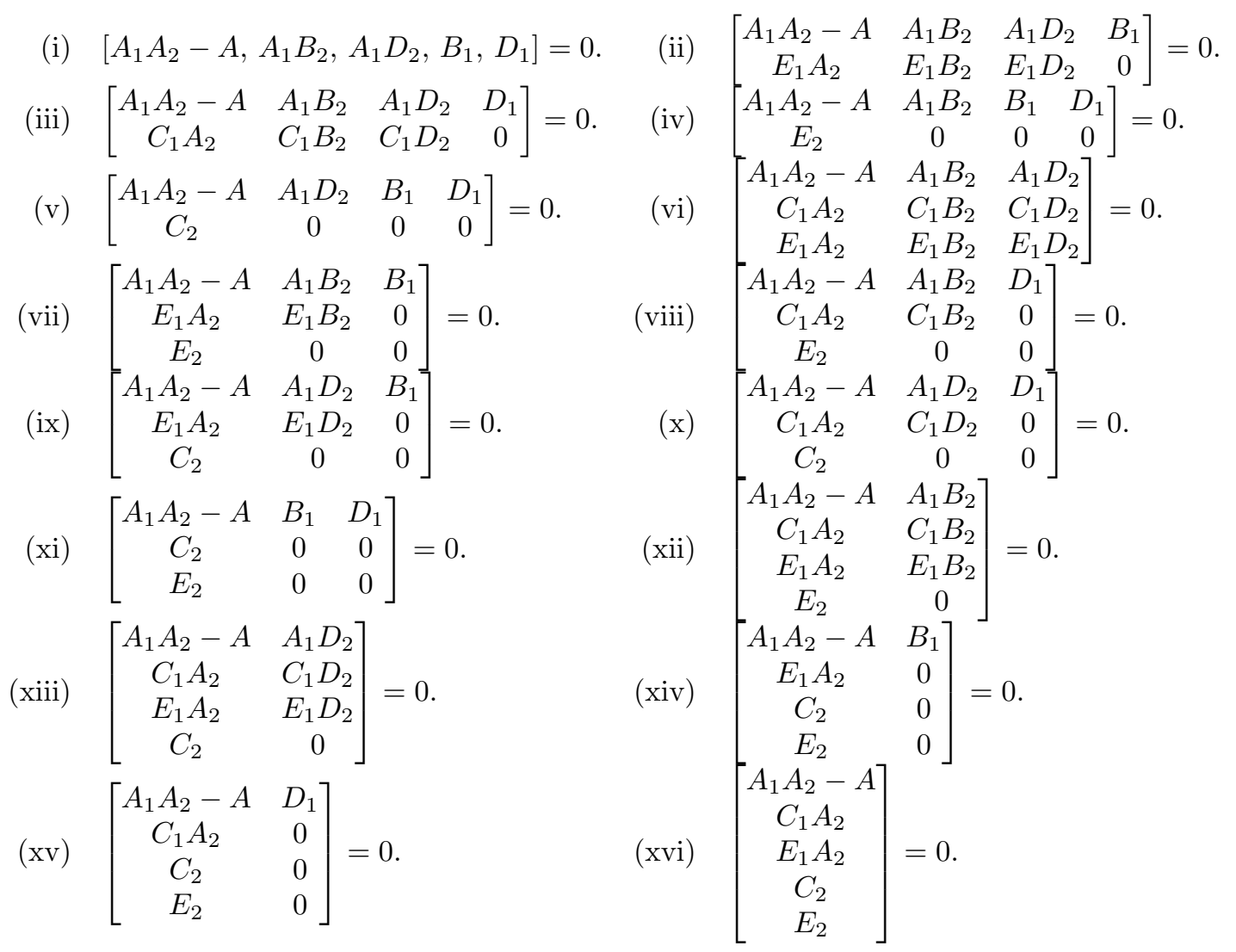

(b) The matrix equation

$$
\left(A_{1}+B_{1} X_{1}+Y_{1} E_{1}\right)\left(A_{2}+B_{2} X_{2}+Y_{2} E_{2}\right)=A
$$

holds for all matrices $X_{1}, X_{2}, Y_{1}$, and $Y_{2}$ if and only if one of the following 3 block matrix equalities holds:

$$
\text { (i) }\left[\begin{array}{ccc}
A & A_{1} & B_{1} \\
0 & E_{1} & 0
\end{array}\right]=0 \text {. (ii) }\left[\begin{array}{cc}
A & 0 \\
A_{2} & B_{2} \\
E_{2} & 0
\end{array}\right]=0 . \quad \text { (iii) }\left[\begin{array}{ccc}
A_{1} A_{2}-A & A_{1} B_{2} & B_{1} \\
E_{1} A_{2} & E_{1} B_{2} & 0 \\
E_{2} & 0 & 0
\end{array}\right]=0 \text {. }
$$

(c) The matrix equation

$$
\left(A_{1}+B_{1} X_{1} C_{1}\right)\left(A_{2}+B_{2} X_{2} C_{2}+D_{2} Y_{2} E_{2}\right)=A
$$


holds for all matrices $X_{1}, X_{2}$, and $Y_{2}$ if and only if one of the following 8 block matrix equalities holds:

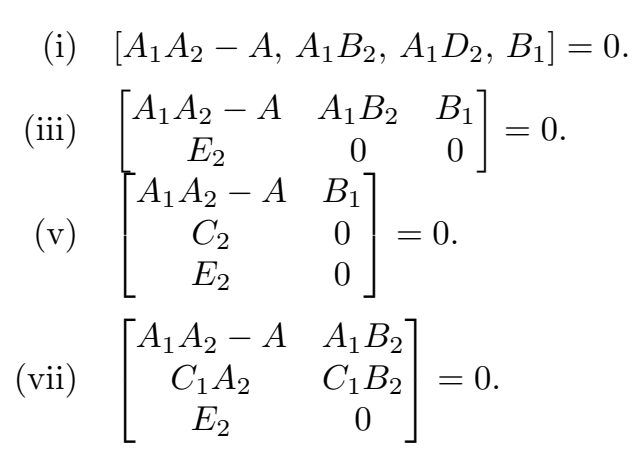

(ii) $\left[\begin{array}{ccc}A_{1} A_{2}-A & A_{1} D_{2} & B_{1} \\ C_{2} & 0 & 0\end{array}\right]=0$.

(iv) $\left[\begin{array}{ccc}A_{1} A_{2}-A & A_{1} B_{2} & A_{1} D_{2} \\ C_{1} A_{2} & C_{1} B_{2} & C_{1} D_{2}\end{array}\right]=0$.

$\left[A_{1} A_{2}-A\right.$

$A_{1} D_{2}$

$$
\left[\begin{array}{cc}
C_{1} A_{2} & C_{1} D_{2} \\
C_{2} & 0
\end{array}\right]=0 .
$$

(d) The matrix equation

$$
\left(A_{1}+B_{1} X_{1} C_{1}+D_{1} Y_{1} E_{1}\right)\left(A_{2}+B_{2} X_{2} C_{2}\right)=A
$$

holds for all matrices $X_{1}, Y_{1}$, and $X_{2}$ if and only if one of the following 8 block matrix equalities holds:
(i) $\left[A_{1} A_{2}-A, A_{1} B_{2}, B_{1}, D_{1}\right]=0$.
(ii) $\left[\begin{array}{ccc}A_{1} A_{2}-A & B_{1} & D_{1} \\ C_{2} & 0 & 0\end{array}\right]=0$.
(iii) $\left[\begin{array}{ccc}A_{1} A_{2}-A & A_{1} B_{2} & B_{1} \\ E_{1} A_{2} & E_{1} B_{2} & 0\end{array}\right]=0$.
(iv) $\left[\begin{array}{ccc}A_{1} A_{2}-A & A_{1} B_{2} & D_{1} \\ C_{1} A_{2} & C_{1} B_{2} & 0\end{array}\right]=0$.
(v) $\left[\begin{array}{cc}A_{1} A_{2}-A & B_{1} \\ E_{1} A_{2} & 0 \\ C_{2} & 0\end{array}\right]=0$.
(vii) $\left[\begin{array}{cc}A_{1} A_{2}-A & A_{1} B_{2} \\ C_{1} A_{2} & C_{1} B_{2} \\ E_{1} A_{2} & E_{1} B_{2}\end{array}\right]=0$.
(viii) $\left[\begin{array}{c}A_{1} A_{2}-A \\ C_{1} A_{2} \\ E_{1} A_{2} \\ C_{2}\end{array}\right]=0$

(e) The bilinear matrix equation

$$
M_{1}\left(A_{1}+B_{1} X_{1}+Y_{1} C_{1}\right) M_{2}\left(A_{2}+B_{2} X_{2}+Y_{2} C_{2}\right) M_{3}=M
$$

holds for all matrices $X_{1}, X_{2}, Y_{1}$, and $Y_{2}$ if and only if one of the following six block matrix equalities holds:
(i) $\left[M, M_{1}\right]=0$,
(ii) $\left[\begin{array}{l}M \\ M_{3}\end{array}\right]=0$
(iii) $\left[\begin{array}{cc}M & 0 \\ 0 & M_{2}\end{array}\right]=0$,
(iv) $\left[\begin{array}{ccc}M & M_{1} A_{1} M_{2} & M_{1} B_{1} \\ 0 & C_{1} M_{2} & 0\end{array}\right]=0$,
(v) $\left[\begin{array}{cc}M & 0 \\ M_{2} A_{2} M_{3} & M_{2} B_{2} \\ C_{2} M_{3} & 0\end{array}\right]=0$,
(vi)

$$
\left[\begin{array}{ccc}
M_{1} A_{1} M_{2} A_{2} M_{3}-M & M_{1} A_{1} M_{2} B_{2} & M_{1} B_{1} \\
C_{1} M_{2} A_{2} M_{3} & C_{1} M_{2} B_{2} & 0 \\
C_{2} M_{3} & 0 & 0
\end{array}\right]=0 .
$$

(f) The matrix equation

$$
\left(A_{1}+B_{1} X_{1} C_{1}\right)\left(A_{2}+B_{2} X_{2}+Y_{2} E_{2}\right)=A
$$

holds for all matrices $X_{1}, X_{2}$, and $Y_{2}$ if and only if one of the following four block matrix equalities holds:
(i) $\left[A, A_{1}, B_{1}\right]=0$.
(ii) $\left[\begin{array}{ccc}A_{1} A_{2}-A & A_{1} B_{2} & B_{1} \\ E_{2} & 0 & 0\end{array}\right]=0$.
(iii) $\left[\begin{array}{cc}A & A_{1} \\ 0 & C_{1}\end{array}\right]=0$.
(iv) $\left[\begin{array}{cc}A_{1} A_{2}-A & A_{1} B_{2} \\ C_{1} A_{2} & C_{1} B_{2} \\ E_{2} & 0\end{array}\right]=0$.

(g) The matrix equation

$$
\left(A_{1}+B_{1} X_{1}+Y_{1} E_{1}\right)\left(A_{2}+B_{2} X_{2} C_{2}\right)=A
$$


holds for all matrices $X_{1}, Y_{1}$, and $X_{2}$ if and only if one of the following four block matrix equalities holds:
(i) $\left[\begin{array}{ccc}A_{1} A_{2}-A & A_{1} B_{2} & B_{1} \\ E_{1} A_{2} & E_{1} B_{2} & 0\end{array}\right]=0$.
(ii) $\left[\begin{array}{cc}A_{1} A_{2}-A & B_{1} \\ E_{1} A_{2} & 0 \\ C_{2} & 0\end{array}\right]=0$.
(iii) $\left[\begin{array}{cc}A & 0 \\ A_{2} & B_{2}\end{array}\right]=0$.
(iv) $\left[\begin{array}{c}A \\ A_{2} \\ C_{2}\end{array}\right]=0$

Lemma 5.8. Let

$$
\left(A_{1}+B_{1} X_{1} C_{1}\right)\left(A_{2}+B_{2} X_{2} C_{2}\right)\left(A_{3}+B_{3} X_{3} C_{3}\right)\left(A_{4}+B_{4} X_{4} C_{4}\right)=A
$$

be a given multilinear matrix equation, where $A, A_{i}, B_{i}$, and $C_{i}$ are known matrices of appropriate sizes, $i=1,2,3,4$. Then the following results hold.

(a) Eq. (5.18) holds for all matrices $X_{1}, X_{2}, X_{3}$, and $X_{4}$ if and only if one of the following 16 block matrix equalities holds:

$$
\begin{aligned}
& \text { (i) }\left[A_{1} A_{2} A_{3} A_{4}-A, A_{1} A_{2} A_{3} B_{4}, A_{1} A_{2} B_{3}, A_{1} B_{2}, B_{1}\right]=0 \text {. } \\
& \text { (ii) }\left[\begin{array}{cccc}
A_{1} A_{2} A_{3} A_{4}-A & A_{1} A_{2} B_{3} & A_{1} B_{2} & B_{1} \\
C_{4} & 0 & 0 & 0
\end{array}\right]=0 \text {. } \\
& \text { (iii) }\left[\begin{array}{cccc}
A_{1} A_{2} A_{3} A_{4}-A & A_{1} A_{2} A_{3} B_{4} & A_{1} B_{2} & B_{1} \\
C_{3} A_{4} & C_{3} B_{4} & 0 & 0
\end{array}\right]=0 \text {. } \\
& \text { (iv) }\left[\begin{array}{cccc}
A_{1} A_{2} A_{3} A_{4}-A & A_{1} A_{2} A_{3} B_{4} & A_{1} A_{2} B_{3} & B_{1} \\
C_{2} A_{3} A_{4} & C_{2} A_{3} B_{4} & C_{2} B_{3} & 0
\end{array}\right]=0 \text {. } \\
& \text { (v) }\left[\begin{array}{cccc}
A_{1} A_{2} A_{3} A_{4}-A & A_{1} A_{2} A_{3} B_{4} & A_{1} A_{2} B_{3} & A_{1} B_{2} \\
C_{1} A_{2} A_{3} A_{4} & C_{1} A_{2} A_{3} B_{4} & C_{1} A_{2} B_{3} & C_{1} B_{2}
\end{array}\right]=0 \text {. } \\
& \text { (vi) }\left[\begin{array}{ccc}
A_{1} A_{2} A_{3} A_{4}-A & A_{1} B_{2} & B_{1} \\
C_{3} A_{4} & 0 & 0 \\
C_{4} & 0 & 0
\end{array}\right]=0 \text {. }
\end{aligned}
$$

(vii) $\quad\left[\begin{array}{ccc}A_{1} A_{2} A_{3} A_{4}-A & A_{1} A_{2} B_{3} & B_{1} \\ C_{2} A_{3} A_{4} & C_{2} B_{3} & 0 \\ C_{4} & 0 & 0\end{array}\right]=0 . \quad$ (viii) $\quad\left[\begin{array}{ccc}A_{1} A_{2} A_{3} A_{4}-A & A_{1} A_{2} B_{3} & A_{1} B_{2} \\ C_{1} A_{2} A_{3} A_{4} & C_{1} A_{2} B_{3} & C_{1} B_{2} \\ C_{4} & 0 & 0\end{array}\right]=0$.

(xi) $\left[\begin{array}{ccc}A_{1} A_{2} A_{3} A_{4}-A & A_{1} A_{2} A_{3} B_{4} & A_{1} A_{2} B_{3} \\ C_{1} A_{2} A_{3} A_{4} & C_{1} A_{2} A_{3} B_{4} & C_{1} A_{2} B_{3} \\ C_{2} A_{3} A_{4} & C_{2} A_{3} B_{4} & C_{2} B_{3}\end{array}\right]=0$.

(xii) $\quad\left[\begin{array}{cc}A_{1} A_{2} A_{3} A_{4}-A & B_{1} \\ C_{2} A_{3} A_{4} & 0 \\ C_{3} A_{4} & 0 \\ C_{4} & 0\end{array}\right]=0$.

(b) Under the assumptions $\mathscr{R}\left(A_{1}\right) \subseteq \mathscr{R}\left(B_{1}\right)$ and $\mathscr{R}\left(A_{4}^{*}\right) \subseteq \mathscr{R}\left(C_{4}^{*}\right)$, (5.18) holds for all matrices $X_{1}, X_{2}, X_{3}$, and 
$X_{4}$ if and only if one of the following 6 block matrix equalities holds:

$$
\begin{aligned}
& \text { (i) }\left[A, B_{1}\right]=0 . \quad \text { (ii) }\left[\begin{array}{c}
A \\
C_{4}
\end{array}\right]=0 \text {. } \\
& \text { (iii) }\left[\begin{array}{cccc}
A_{1} A_{2} A_{3} A_{4}-A & A_{1} A_{2} A_{3} B_{4} & A_{1} A_{2} B_{3} & A_{1} B_{2} \\
C_{1} A_{2} A_{3} A_{4} & C_{1} A_{2} A_{3} B_{4} & C_{1} A_{2} B_{3} & C_{1} B_{2}
\end{array}\right]=0 \text {. } \\
& \text { (iv) } \quad\left[\begin{array}{ccc}
A_{1} A_{2} A_{3} A_{4}-A & A_{1} A_{2} A_{3} B_{4} & A_{1} B_{2} \\
C_{1} A_{2} A_{3} A_{4} & C_{1} A_{2} A_{3} B_{4} & C_{1} B_{2}
\end{array}\right]=0 \text {. } \\
& \text { (v) }\left[\begin{array}{ccc}
A_{1} A_{2} A_{3} A_{4}-A & A_{1} A_{2} A_{3} B_{4} & A_{1} A_{2} B_{3} \\
C_{1} A_{2} A_{3} A_{4} & C_{1} A_{2} A_{3} B_{4} & C_{1} A_{2} B_{3} \\
C_{2} A_{3} A_{4} & C_{2} A_{3} B_{4} & C_{2} B_{3}
\end{array}\right]=0 . \\
& \text { (vi) }\left[\begin{array}{cc}
C_{1} A_{2} A_{3} A_{4} & C_{1} A_{2} A_{3} B_{4} \\
C_{2} A_{3} A_{4} & C_{2} A_{3} B_{4} \\
C_{3} A_{4} & C_{3} B_{4}
\end{array}\right]=0
\end{aligned}
$$

(c) Under the assumption $\mathscr{R}\left(A_{1}\right) \subseteq \mathscr{R}\left(B_{1}\right), \mathscr{R}\left(A_{2}^{*}\right) \subseteq \mathscr{R}\left(C_{2}^{*}\right), \mathscr{R}\left(A_{3}\right) \subseteq \mathscr{R}\left(B_{3}\right)$, and $\mathscr{R}\left(A_{4}^{*}\right) \subseteq \mathscr{R}\left(C_{4}^{*}\right),(5.18)$ holds for all matrices $X_{1}, X_{2}, X_{3}$, and $X_{4}$ if and only if one of the following 6 block matrix equalities holds:

$$
\begin{aligned}
& \text { (i) }\left[A, B_{1}\right]=0 . \quad \text { (ii) }\left[\begin{array}{c}
A \\
C_{4}
\end{array}\right]=0 . \quad \text { (iii) }\left[\begin{array}{cc}
A & 0 \\
0 & C_{2} B_{3}
\end{array}\right]=0 . \\
& \text { (iv) }\left[\begin{array}{ccc}
A & A_{1} A_{2} B_{3} & A_{1} B_{2} \\
0 & C_{1} A_{2} B_{3} & C_{1} B_{2}
\end{array}\right]=0 . \quad \text { (v) }\left[\begin{array}{cc}
A & 0 \\
C_{2} A_{3} A_{4} & C_{2} A_{3} B_{4} \\
C_{3} A_{4} & C_{3} B_{4}
\end{array}\right]=0 \text {. } \\
& \text { (vi) }\left[\begin{array}{ccc}
A_{1} A_{2} A_{3} A_{4}-A & A_{1} A_{2} A_{3} B_{4} & A_{1} B_{2} \\
C_{1} A_{2} A_{3} A_{4} & C_{1} A_{2} A_{3} B_{4} & C_{1} B_{2} \\
C_{3} A_{4} & C_{3} B_{4} & 0
\end{array}\right]=0
\end{aligned}
$$

(d) The multilinear matrix equation

$$
M_{1}\left(A_{1}+B_{1} X_{1}\right) M_{2}\left(A_{2}+X_{2} C_{2}\right) M_{3}\left(A_{3}+B_{3} X_{3}\right) M_{4}\left(A_{4}+X_{4} C_{4}\right) M_{5}=A
$$

holds for all variable matrices $X_{1}, X_{2}, X_{3}$, and $X_{4}$ if and only if one of the following eight block matrix equalities holds:

$$
\begin{aligned}
& \text { (i) }\left[A, M_{1} A_{1} M_{2}, M_{1} B_{1}\right]=0 \text {, } \\
& \text { (ii) }\left[\begin{array}{cc}
A & 0 \\
0 & M_{2}
\end{array}\right]=0, \quad \text { (iii) }\left[\begin{array}{cc}
A & 0 \\
0 & M_{4}
\end{array}\right]=0 \text {, } \\
& \text { (v) }\left[\begin{array}{cccc}
A & M_{1} A_{1} M_{2} A_{2} M_{3} A_{3} M_{4} & M_{1} A_{1} M_{2} A_{2} M_{3} B_{3} & M_{1} B_{1} \\
0 & C_{2} M_{3} A_{3} M_{4} & C_{2} M_{3} B_{3} & 0
\end{array}\right]=0 \text {, }
\end{aligned}
$$

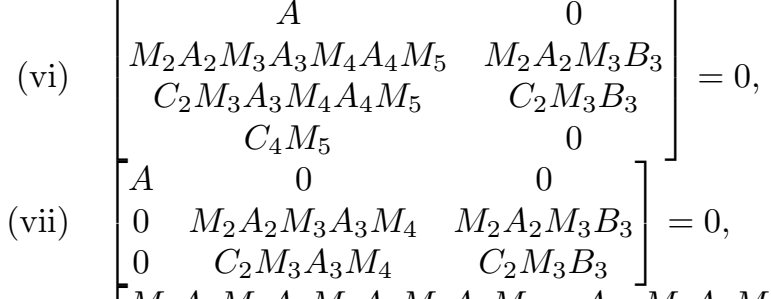

$$
\begin{aligned}
& \text { (viii) }\left[\begin{array}{ccc}
M_{1} A_{1} M_{2} A_{2} M_{3} A_{3} M_{4} A_{4} M_{5}-A & M_{1} A_{1} M_{2} A_{2} M_{3} B_{3} & M_{1} B_{1} \\
C_{2} M_{3} A_{3} M_{4} A_{4} M_{5} & C_{2} M_{3} B_{3} & 0 \\
C_{4} M_{5} & 0 & 0
\end{array}\right]=0 \text {. }
\end{aligned}
$$

Obviously, the block matrix equalities given in Lemmas $5.2,5.8$ reveal some essential connections among the products of the given matrices in the linear and multilinear matrix equations, which therefore show many beautiful facets of linear and multilinear matrix identities that involve variable matrices. These lemmas in fact provide a set of highly efficient methods to establish and verify many types of matrix equality, and can be used to characterize the relationships between matrix sets composed by matrix-valued functions. 


\section{Invariance property of matrix products that involve two generalized inverses}

The hundreds of matrix-valued functions in 3.89-3.187) show in a striking manner what are the difficulties in the classification and characterization of the reverse order law problems formulated in (1.7)-(1.11). In order words, $\mathrm{w}$ need to make considerable extra efforts from the very beginning in order to obtain a complete set of solutions to all these reverse order law problems. For a matrix expression that involves generalized inverses, one of the most fundamental fact people like to know is concerned with the invariance property of the matrix expression with respect to the choice of the generalized inverses; see e.g., $[7,37,55,58$ for expositions and some previous results. We have seen from Lemma $3.3(\mathrm{~h})$ that the invariance property (uniqueness) of the product $B^{\left(s_{2}, \ldots, t_{2}\right)} A^{\left(s_{1}, \ldots, t_{1}\right)}$ occurs in the study of the ROLs $(A B)^{\dagger}=B^{\left(s_{2}, \ldots, t_{2}\right)} A^{\left(s_{1}, \ldots, t_{1}\right)}$. So that we need to know necessary and sufficient conditions for the product $B^{\left(s_{2}, \ldots, t_{2}\right)} A^{\left(s_{1}, \ldots, t_{1}\right)}$ to be invariant with respect to choice of $A^{\left(s_{1}, \ldots, t_{1}\right)}$ and $B^{\left(s_{2}, \ldots, t_{2}\right)}$. Because $B^{\dagger} A^{\dagger}$ is unique once $A$ and $B$ are given, we can readily see from the definition of the Moore-Penrose inverse that the product $B^{\left(s_{2}, \ldots, t_{2}\right)} A^{\left(s_{1}, \ldots, t_{1}\right)}$ is invariant with respect to the choice of the eight commonly-used types of generalized inverses $A^{\left(s_{1}, \ldots, t_{1}\right)}$ and $B^{\left(s_{2}, \ldots, t_{2}\right)}$ if and only if the equality

$$
B^{\left(s_{2}, \ldots, t_{2}\right)} A^{\left(s_{1}, \ldots, t_{1}\right)}=B^{\dagger} A^{\dagger}
$$

holds for all $A^{\left(s_{1}, \ldots, t_{1}\right)}$ and $B^{\left(s_{2}, \ldots, t_{2}\right)}$. Substituting the 63 matrix-valued functions in (3.89)- 3.151) into 6.1), respectively, will result in 63 linear or multilinear matrix equations. In this section, we shall only present the detailed results concerning the invariance property of 63 matrix equations in (6.1). The complete characterizations of these invariance property problems can be done by means of Lemmas 5.2-5.8, but the details are rather technical and tedious, and are therefore omitted.

Theorem 6.1. Given $A \in \mathbb{C}^{m \times n}$ and $B \in \mathbb{C}^{n \times p}$, we have the following results:

$\langle 1\rangle B^{\dagger} A^{(1,3,4)}$ is invariant $\Leftrightarrow r(A)=m$ or $\mathscr{R}\left(A^{*}\right) \supseteq \mathscr{R}(B)$.

$\langle 2\rangle B^{\dagger} A^{(1,2,4)}$ is invariant $\Leftrightarrow A B=0$ or $r(A)=m$.

$\langle 3\rangle B^{\dagger} A^{(1,2,3)}$ is invariant $\Leftrightarrow A=0$ or $\mathscr{R}\left(A^{*}\right) \supseteq \mathscr{R}(B)$.

$\langle 4\rangle B^{\dagger} A^{(1,4)}$ is invariant $\Leftrightarrow r(A)=m$ or $B=0$.

$\langle 5\rangle B^{\dagger} A^{(1,3)}$ is invariant $\Leftrightarrow \mathscr{R}\left(A^{*}\right) \supseteq \mathscr{R}(B)$.

$\langle 6\rangle B^{\dagger} A^{(1,2)}$ is invariant $\Leftrightarrow A=0$ or $B=0$ or $\left\{r(A)=m\right.$ and $\left.\mathscr{R}\left(A^{*}\right) \supseteq \mathscr{R}(B)\right\}$.

$\langle 7\rangle B^{\dagger} A^{(1)}$ is invariant $\Leftrightarrow B=0$ or $\left\{r(A)=m\right.$ and $\left.\mathscr{R}\left(A^{*}\right) \supseteq \mathscr{R}(B)\right\}$.

$\langle 8\rangle B^{(1,3,4)} A^{\dagger}$ is invariant $\Leftrightarrow$ either $r(B)=$ p or $\mathscr{R}\left(A^{*}\right) \subseteq \mathscr{R}(B)$.

$\langle 9\rangle B^{(1,3,4)} A^{(1,3,4)}$ is invariant $\Leftrightarrow r(A)=r(B)=n$ or $\{r(A)=m$ and $r(B)=p\}$ or $\left\{r(A)=m\right.$ and $\mathscr{R}\left(A^{*}\right) \subseteq$ $\mathscr{R}(B)\}$ or $\left\{r(B)=p\right.$ and $\left.\mathscr{R}\left(A^{*}\right) \supseteq \mathscr{R}(B)\right\}$.

$\langle 10\rangle B^{(1,3,4)} A^{(1,2,4)}$ is invariant $\Leftrightarrow\{A B=0$ and $r(B)=p\}$ or $\{r(A)=m$ and $r(B)=p\}$ or $\{A B=0$ and $\left.\mathscr{R}\left(A^{*}\right) \subseteq \mathscr{R}(B)\right\}$ or $\left\{r(A)=m\right.$ and $\left.\mathscr{R}\left(A^{*}\right) \subseteq \mathscr{R}(B)\right\}$

$\langle 11\rangle B^{(1,3,4)} A^{(1,2,3)}$ is invariant $\Leftrightarrow A=0$ or $\left\{r(B)=p\right.$ and $\left.\mathscr{R}\left(A^{*}\right) \supseteq \mathscr{R}(B)\right\}$ or $r(A)=r(B)=n$.

$\langle 12\rangle B^{(1,3,4)} A^{(1,4)}$ is invariant $\Leftrightarrow\{r(A)=m$ and $r(B)=p\}$ or $\left\{r(A)=m\right.$ and $\left.\mathscr{R}\left(A^{*}\right) \subseteq \mathscr{R}(B)\right\}$.

$\langle 13\rangle B^{(1,3,4)} A^{(1,3)}$ is invariant $\Leftrightarrow r(A)=r(B)=n$ or $\left\{r(B)=p\right.$ and $\left.\mathscr{R}\left(A^{*}\right) \supseteq \mathscr{R}(B)\right\}$.

$\langle 14\rangle B^{(1,3,4)} A^{(1,2)}$ is invariant $\Leftrightarrow A=0$ or $r(A)=r(B)=m=n$ or $\{A B=0, r(A)=m$, and $r(B)=p\}$.

$\langle 15\rangle B^{(1,3,4)} A^{(1)}$ is invariant $\Leftrightarrow r(A)=r(B)=m=n$ or $\left\{r(A)=m, r(B)=p\right.$, and $\left.\mathscr{R}\left(A^{*}\right) \supseteq \mathscr{R}(B)\right\}$.

$\langle 16\rangle B^{(1,2,4)} A^{\dagger}$ is invariant $\Leftrightarrow B=0$ or $\mathscr{R}\left(A^{*}\right) \subseteq \mathscr{R}(B)$.

$\langle 17\rangle B^{(1,2,4)} A^{(1,3,4)}$ is invariant $\Leftrightarrow B=0$ or $\left\{r(A)=m\right.$ and $\left.\mathscr{R}\left(A^{*}\right) \subseteq \mathscr{R}(B)\right\}$ or $r(A)=r(B)=n$.

$\langle 18\rangle B^{(1,2,4)} A^{(1,2,4)}$ is invariant $\Leftrightarrow A=0$ or $B=0$ or $\left\{r(A)=m\right.$ and $\left.\mathscr{R}\left(A^{*}\right) \subseteq \mathscr{R}(B)\right\}$.

$\langle 19\rangle B^{(1,2,4)} A^{(1,2,3)}$ is invariant $\Leftrightarrow A=0$ or $B=0$ or $r(A)=r(B)=n$.

$\langle 20\rangle B^{(1,2,4)} A^{(1,4)}$ is invariant $\Leftrightarrow B=0$ or $\left\{r(A)=m\right.$ and $\left.\mathscr{R}\left(A^{*}\right) \subseteq \mathscr{R}(B)\right\}$. 
$\langle 21\rangle B^{(1,2,4)} A^{(1,3)}$ is invariant $\Leftrightarrow B=0$ or $r(A)=r(B)=n$.

$\langle 22\rangle B^{(1,2,4)} A^{(1,2)}$ is invariant $\Leftrightarrow A=0$ or $B=0$ or $r(A)=r(B)=m=n$.

$\langle 23\rangle B^{(1,2,4)} A^{(1)}$ is invariant $\Leftrightarrow B=0$ or $r(A)=r(B)=m=n$.

$\langle 24\rangle B^{(1,2,3)} A^{\dagger}$ is invariant $\Leftrightarrow A=0$ or $B=0$ or $(B)=p$.

$\langle 25\rangle B^{(1,2,3)} A^{(1,3,4)}$ is invariant $\Leftrightarrow\{A B=0$ and $r(A)=m\}$ or $\{r(A)=m$ and $r(B)=p\}$ or $\{A B=0$ and $\left.\mathscr{R}\left(A^{*}\right) \supseteq \mathscr{R}(B)\right\}$ or $\left\{r(B)=p\right.$ and $\left.\mathscr{R}\left(A^{*}\right) \supseteq \mathscr{R}(B)\right\}$.

$\langle 26\rangle B^{(1,2,3)} A^{(1,2,4)}$ is invariant $\Leftrightarrow A B=0$ or $\{r(A)=m$ and $r(B)=p\}$.

$\langle 27\rangle B^{(1,2,3)} A^{(1,2,3)}$ is invariant $\Leftrightarrow A=0$ or $B=0$ or $\left\{r(B)=p\right.$ and $\left.\mathscr{R}\left(A^{*}\right) \supseteq \mathscr{R}(B)\right\}$.

$\langle 28\rangle B^{(1,2,3)} A^{(1,4)}$ is invariant $\Leftrightarrow B=0$ or $\{A B=0$ and $r(A)=m\}$ or $\{r(A)=m$ and $r(B)=p\}$.

$\langle 29\rangle B^{(1,2,3)} A^{(1,3)}$ is invariant $\Leftrightarrow B=0$ or $\left\{r(B)=p\right.$ and $\left.\mathscr{R}\left(A^{*}\right) \supseteq \mathscr{R}(B)\right\}$.

$\langle 30\rangle B^{(1,2,3)} A^{(1,2)}$ is invariant $\Leftrightarrow A=0$ or $B=0$ or $\left\{r(A)=m, r(B)=p\right.$, and $\left.\mathscr{R}\left(A^{*}\right) \supseteq \mathscr{R}(B)\right\}$.

$\langle 31\rangle B^{(1,2,3)} A^{(1)}$ is invariant $\Leftrightarrow B=0$ or $\left\{r(A)=m, r(B)=p\right.$, and $\left.\mathscr{R}\left(A^{*}\right) \supseteq \mathscr{R}(B)\right\}$.

$\langle 32\rangle B^{(1,4)} A^{\dagger}$ is invariant $\Leftrightarrow \mathscr{R}\left(A^{*}\right) \subseteq \mathscr{R}(B)$.

$\langle 33\rangle B^{(1,4)} A^{(1,3,4)}$ is invariant $\Leftrightarrow r(A)=r(B)=n$ or $\left\{r(A)=m\right.$ and $\left.\mathscr{R}\left(A^{*}\right) \subseteq \mathscr{R}(B)\right\}$.

$\langle 34\rangle B^{(1,4)} A^{(1,2,4)}$ is invariant $\Leftrightarrow A=0$ or $\left\{r(A)=m\right.$ and $\left.\mathscr{R}\left(A^{*}\right) \subseteq \mathscr{R}(B)\right\}$.

$\langle 35\rangle B^{(1,4)} A^{(1,2,3)}$ is invariant $\Leftrightarrow A=0$ or $r(A)=r(B)=n$.

$\langle 36\rangle B^{(1,4)} A^{(1,4)}$ is invariant $\Leftrightarrow r(A)=m$ and $\mathscr{R}\left(A^{*}\right) \subseteq \mathscr{R}(B)$.

$\langle 37\rangle B^{(1,4)} A^{(1,3)}$ is invariant $\Leftrightarrow r(A)=r(B)=n$.

$\langle 38\rangle B^{(1,4)} A^{(1,2)}$ is invariant $\Leftrightarrow A=0$ or $r(A)=r(B)=m=n$.

$\langle 39\rangle B^{(1,4)} A^{(1)}$ is invariant $\Leftrightarrow r(A)=r(B)=m=n$.

$\langle 40\rangle B^{(1,3)} A^{\dagger}$ is invariant $\Leftrightarrow r(B)=p$.

$\langle 41\rangle B^{(1,3)} A^{(1,3,4)}$ is invariant $\Leftrightarrow\{r(A)=m$ and $r(B)=p\}$ or $\left\{r(B)=p\right.$ and $\left.\mathscr{R}\left(A^{*}\right) \supseteq \mathscr{R}(B)\right\}$.

$\langle 42\rangle B^{(1,3)} A^{(1,2,4)}$ is invariant $\Leftrightarrow A=0$ or $\{A B=0$ and $r(B)=p\}$ or $\{r(A)=m$ and $r(B)=p\}$.

$\langle 43\rangle B^{(1,3)} A^{(1,2,3)}$ is invariant $\Leftrightarrow A=0$ or $\left\{r(B)=p\right.$ and $\left.\mathscr{R}\left(A^{*}\right) \supseteq \mathscr{R}(B)\right\}$.

$\langle 44\rangle B^{(1,3)} A^{(1,4)}$ is invariant $\Leftrightarrow r(A)=m$ and $r(B)=p$.

$\langle 45\rangle B^{(1,3)} A^{(1,3)}$ is invariant $\Leftrightarrow r(B)=p$ and $\mathscr{R}\left(A^{*}\right) \supseteq \mathscr{R}(B)$.

$\langle 46\rangle B^{(1,3)} A^{(1,2)}$ is invariant $\Leftrightarrow A=0$ or $\left\{r(A)=m, r(B)=p\right.$, and $\left.\mathscr{R}\left(A^{*}\right) \supseteq \mathscr{R}(B)\right\}$.

$\langle 47\rangle B^{(1,3)} A^{(1)}$ is invariant $\Leftrightarrow r(A)=m, r(B)=p$, and $\mathscr{R}\left(A^{*}\right) \supseteq \mathscr{R}(B)$.

$\langle 48\rangle B^{(1,2)} A^{\dagger}$ is invariant $\Leftrightarrow A=0$ or $B=0$ or $\left\{r(B)=p\right.$ and $\left.\mathscr{R}\left(A^{*}\right) \subseteq \mathscr{R}(B)\right\}$.

$\langle 49\rangle B^{(1,2)} A^{(1,3,4)}$ is invariant $\Leftrightarrow B=0$ or $r(A)=r(B)=n=p$ or $\{A B=0, r(A)=m$, and $r(B)=p\}$.

$\langle 50\rangle B^{(1,2)} A^{(1,2,4)}$ is invariant $\Leftrightarrow A=0$ or $B=0$ or $\left\{r(A)=m, r(B)=p\right.$, and $\left.\mathscr{R}\left(A^{*}\right) \subseteq \mathscr{R}(B)\right\}$.

$\langle 51\rangle B^{(1,2)} A^{(1,2,3)}$ is invariant $\Leftrightarrow A=0$ or $B=0$ or $r(A)=r(B)=n=p$.

$\langle 52\rangle B^{(1,2)} A^{(1,4)}$ is invariant $\Leftrightarrow B=0$ or $\left\{r(A)=m, r(B)=p\right.$, and $\left.\mathscr{R}\left(A^{*}\right) \subseteq \mathscr{R}(B)\right\}$.

$\langle 53\rangle B^{(1,2)} A^{(1,3)}$ is invariant $\Leftrightarrow B=0$ or $r(A)=r(B)=n=p$.

$\langle 54\rangle B^{(1,2)} A^{(1,2)}$ is invariant $\Leftrightarrow A=0$ or $B=0$ or $r(A)=r(B)=m=n=p$.

$\langle 55\rangle B^{(1,2)} A^{(1)}$ is invariant $\Leftrightarrow B=0$ or $r(A)=r(B)=m=n=p$.

$\langle 56\rangle B^{(1)} A^{\dagger}$ is invariant $\Leftrightarrow r(B)=p$ and $\mathscr{R}\left(A^{*}\right) \subseteq \mathscr{R}(B)$. 
$\langle 57\rangle B^{(1)} A^{(1,3,4)}$ is invariant $\Leftrightarrow r(A)=r(B)=n=p$ or $\left\{r(A)=m, r(B)=p\right.$, and $\left.\mathscr{R}\left(A^{*}\right) \subseteq \mathscr{R}(B)\right\}$.

$\langle 58\rangle B^{(1)} A^{(1,2,4)}$ is invariant $\Leftrightarrow A=0$ or $\left\{r(A)=m, r(B)=p\right.$, and $\left.\mathscr{R}\left(A^{*}\right) \subseteq \mathscr{R}(B)\right\}$.

$\langle 59\rangle B^{(1)} A^{(1,2,3)}$ is invariant $\Leftrightarrow r(A)=r(B)=n=p$.

$\langle 60\rangle B^{(1)} A^{(1,4)}$ is invariant $\Leftrightarrow r(A)=m, r(B)=p$, and $\mathscr{R}\left(A^{*}\right) \subseteq \mathscr{R}(B)$.

$\langle 61\rangle B^{(1)} A^{(1,3)}$ is invariant $\Leftrightarrow r(A)=r(B)=n=p$.

$\langle 62\rangle B^{(1)} A^{(1,2)}$ is invariant $\Leftrightarrow A=0$ or $r(A)=r(B)=m=n=p$.

$\langle 63\rangle B^{(1)} A^{(1)}$ is invariant $\Leftrightarrow r(A)=r(B)=m=n=p$.

Theorem 6.2. Given $A \in \mathbb{C}^{m \times n}$ and $B \in \mathbb{C}^{n \times p}$, and denote $N=\left[A^{*}, B\right]$, we have the following results:

$\langle 1\rangle$ The products in 3.153 are invariant $\Leftrightarrow r(A B)=r(A)+r(B)-r(N)$.

$\langle 2\rangle$ The products in 3.154 are invariant $\Leftrightarrow r(A B)=r(A)+r(B)-r(N)$.

$\langle 3\rangle$ The products in 3.155 are invariant $\Leftrightarrow r(A B)=r(A)+r(B)-n$.

$\langle 4\rangle$ The products in 3.157 are invariant $\Leftrightarrow r(A)=m$ or $r(A B)=r(A)+r(B)-r(N)$.

$\langle 5\rangle$ The products in 3.158 are invariant $\Leftrightarrow A B=0$ or $r(A)=m$.

$\langle 6\rangle$ The products in 3.159 are invariant $\Leftrightarrow r(A B)=r(A)+r(B)-r(N)$.

$\langle 7\rangle$ The products in 3.160 are invariant $\Leftrightarrow A B=0$ or $r(A)=m$.

$\langle 8\rangle$ The products in 3.161 are invariant $\Leftrightarrow r(A B)=r(A)+r(B)-r(N)$.

$\langle 9\rangle$ The products in 3.162 are invariant $\Leftrightarrow r(A)=m$ or $r(A B)=r(A)+r(B)-r(N)$.

$\langle 10\rangle$ The products in 3.163 are invariant $\Leftrightarrow r(A)=m$ or $r(A B)=r(A)+r(B)-r(N)$.

$\langle 11\rangle$ The products in 3.164 are invariant $\Leftrightarrow A B=0$ or $\mathscr{R}\left(A^{*}\right) \subseteq \mathscr{R}(B)$.

$\langle 12\rangle$ The products in 3.165 are invariant $\Leftrightarrow A B=0$ or $\left\{r(A B)=n\right.$ or $r(A)=m$ and $\left.\mathscr{R}\left(A^{*}\right) \subseteq \mathscr{R}(B)\right\}$.

$\langle 13\rangle$ The products in 3.166 are invariant $\Leftrightarrow A B=0$ or $\left\{r(A)=m\right.$ and $\left.\mathscr{R}\left(A^{*}\right) \subseteq \mathscr{R}(B)\right\}$.

$\langle 14\rangle$ The products in 3.167 are invariant $\Leftrightarrow r(A)=r(B)=n$.

$\langle 15\rangle$ The products in 3.168 are invariant $\Leftrightarrow A B=0$ or $\left\{r(A)=m\right.$ and $\left.\mathscr{R}\left(A^{*}\right) \subseteq \mathscr{R}(B)\right\}$.

$\langle 16\rangle$ The products in 3.169 are invariant $\Leftrightarrow A B=0$ or $r(A)=r(B)=n$.

$\langle 17\rangle$ The products in 3.170 are invariant $\Leftrightarrow A B=0$ or $r(A)=r(B)=m=n$.

$\langle 18\rangle$ The products in 3.171 are invariant $\Leftrightarrow A B=0$ or $r(A)=r(B)=m=n$.

$\langle 20\rangle$ The products in 3.173 are invariant $\Leftrightarrow$ either $r(B)=p$ or $r(A B)=r(A)+r(B)-r(N)$.

$\langle 21\rangle$ The products in 3.174 are invariant $\Leftrightarrow r(N)=r(A)+r(B)-r(A B)$.

$\langle 22\rangle$ The products in 3.175 are invariant $\Leftrightarrow A B=0$ or $r(B)=p$.

$\langle 23\rangle$ The products in 3.176 are invariant $\Leftrightarrow r(A B)=r(A)+r(B)-r(N)$.

$\langle 24\rangle$ The products in 3.177 are invariant $\Leftrightarrow A B=0$ or $r(B)=p$.

$\langle 25\rangle$ The products in 3.178 are invariant $\Leftrightarrow r(B)=p$ or $r(A B)=r(A)+r(B)-r(N)$.

$\langle 26\rangle$ The products in 3.179 are invariant $\Leftrightarrow r(B)=p$ or $r(A B)=r(A)+r(B)-r(N)$.

$\langle 27\rangle$ The products in 3.180 are invariant $\Leftrightarrow A B=0$ or $\mathscr{R}\left(A^{*}\right) \supseteq \mathscr{R}(B)$.

$\langle 28\rangle$ The products in 3.181 are invariant $\Leftrightarrow A B=0$ or $r(A)=r(B)=n$ or $\left\{r(B)=p\right.$ and $\left.\mathscr{R}\left(A^{*}\right) \supseteq \mathscr{R}(B)\right\}$.

$\langle 29\rangle$ The products in 3.182 are invariant $\Leftrightarrow A B=0$ or $r(A)=r(B)=n$.

$\langle 30\rangle$ The products in 3.183 are invariant $\Leftrightarrow A B=0$ or $\left\{r(B)=p\right.$ and $\left.\mathscr{R}\left(A^{*}\right) \supseteq \mathscr{R}(B)\right\}$. 
$\langle 31\rangle$ The products in 3.184 are invariant $\Leftrightarrow A B=0$ or $r(A)=r(B)=n$.

$\langle 32\rangle$ The products in 3.185 are invariant $\Leftrightarrow A B=0$ or $\left\{r(B)=p\right.$ and $\left.\mathscr{R}\left(A^{*}\right) \supseteq \mathscr{R}(B)\right\}$.

$\langle 33\rangle$ The products in (3.186) are invariant $\Leftrightarrow A B=0$ or $r(A)=r(B)=n=p$.

$\langle 34\rangle$ The products in (3.187) are invariant $\Leftrightarrow A B=0$ or $r(A)=r(B)=n=p$.

\section{Fundamental equalities between generalized inverses of two matrices}

The simplest matrix equalities for generalized inverses in $(1.4)$ are given by $A^{(i, \ldots, j)}=B^{\left(s_{2}, \ldots, t_{2}\right)}$. Necessary and sufficient conditions for these equalities to hold are currently being worked out by the present author in [90] as follows.

Lemma 7.1. Given $A, B \in \mathbb{C}^{m \times n}$, we have the following 24 results:

$\langle 1\rangle A^{\dagger}=B^{\dagger} \Leftrightarrow A=B$.

$\langle 2\rangle A^{\dagger} \in\left\{B^{(1,3,4)}\right\} \Leftrightarrow B^{*} A=B^{*} B$ and $A B^{*}=B B^{*}$.

$\langle 3\rangle A^{\dagger} \in\left\{B^{(1,2,4)}\right\} \Leftrightarrow \mathscr{R}\left(A^{*}\right)=\mathscr{R}\left(B^{*}\right)$ and $A^{*} A=A^{*} B$.

$\langle 4\rangle A^{\dagger} \in\left\{B^{(1,4)}\right\} \Leftrightarrow \mathscr{R}\left(A^{*}\right) \supseteq \mathscr{R}\left(B^{*}\right)$ and $A^{*} A B^{*}=A^{*} B B^{*}$.

$\langle 5\rangle A^{\dagger} \in\left\{B^{(1,2)}\right\} \Leftrightarrow r(A)=r(B)$ and $A^{*} A A^{*}=A^{*} B A^{*}$.

$\langle 6\rangle A^{\dagger} \in\left\{B^{(1)}\right\} \Leftrightarrow r\left(A^{*} A A^{*}-A^{*} B A^{*}\right)=r(A)-r(B)$.

$\langle 7\rangle\left\{A^{(1,3,4)}\right\} \cap\left\{B^{(1,3,4)}\right\} \neq \emptyset \Leftrightarrow \mathscr{R}\left[\begin{array}{l}A \\ B\end{array}\right] \subseteq \mathscr{R}\left[\begin{array}{l}A A^{*} \\ B B^{*}\end{array}\right], \mathscr{R}\left[\begin{array}{l}A^{*} \\ B^{*}\end{array}\right] \subseteq \mathscr{R}\left[\begin{array}{l}A^{*} A \\ B^{*} B\end{array}\right], A^{*} A B^{*}=A^{*} B B^{*}$, and $B^{*} A A^{*}=$ $B^{*} B A^{*}$.

$\langle 8\rangle\left\{A^{(1,3,4)}\right\} \cap\left\{B^{(1,2,4)}\right\} \neq \emptyset \Leftrightarrow \mathscr{R}\left[\begin{array}{l}A \\ B\end{array}\right] \subseteq \mathscr{R}\left[\begin{array}{l}A A^{*} \\ B B^{*}\end{array}\right]$ and $A^{*} A=A^{*} B$.

$\langle 9\rangle\left\{A^{(1,3,4)}\right\} \cap\left\{B^{(1,4)}\right\} \neq \emptyset \Leftrightarrow \mathscr{R}\left[\begin{array}{l}A \\ B\end{array}\right] \subseteq \mathscr{R}\left[\begin{array}{l}A A^{*} \\ B B^{*}\end{array}\right]$ and $A^{*} A B^{*}=A^{*} B B^{*}$.

$\langle 10\rangle\left\{A^{(1,3,4)}\right\} \cap\left\{B^{(1,2)}\right\} \neq \emptyset \Leftrightarrow \mathscr{R}\left[\begin{array}{c}A^{*} B \\ B\end{array}\right] \subseteq \mathscr{R}\left[\begin{array}{c}A^{*} A \\ B\end{array}\right], \mathscr{R}\left[\begin{array}{c}A B^{*} \\ B^{*}\end{array}\right] \subseteq \mathscr{R}\left[\begin{array}{c}A A^{*} \\ B^{*}\end{array}\right]$, and $A^{*} A A^{*}=A^{*} B A^{*}$

$\langle 11\rangle\left\{A^{(1,3,4)}\right\} \cap\left\{B^{(1)}\right\} \neq \emptyset \Leftrightarrow \mathscr{R}\left[\begin{array}{c}A^{*} B \\ B\end{array}\right] \subseteq \mathscr{R}\left[\begin{array}{c}A^{*} A \\ B\end{array}\right]$ and $\mathscr{R}\left[\begin{array}{c}A B^{*} \\ B^{*}\end{array}\right] \subseteq \mathscr{R}\left[\begin{array}{c}A A^{*} \\ B^{*}\end{array}\right]$.

$\langle 12\rangle\left\{A^{(1,2,4)}\right\} \cap\left\{B^{(1,2,4)}\right\} \neq \emptyset \Leftrightarrow \mathscr{R}\left(A^{*}\right)=\mathscr{R}\left(B^{*}\right)$ and $\mathscr{R}\left[\begin{array}{l}A \\ B\end{array}\right] \subseteq \mathscr{R}\left[\begin{array}{l}A A^{*} \\ B B^{*}\end{array}\right]$.

$\langle 13\rangle\left\{A^{(1,2,4)}\right\} \cap\left\{B^{(1,2,3)}\right\} \neq \emptyset \Leftrightarrow r\left(A B^{*}\right)=r\left(B^{*} A\right)=r(A)=r(B)$ and $B^{*} A A^{*}=B^{*} B A^{*}$.

$\langle 14\rangle\left\{A^{(1,2,4)}\right\} \cap\left\{B^{(1,4)}\right\} \neq \emptyset \Leftrightarrow \mathscr{R}\left(A^{*}\right) \supseteq \mathscr{R}\left(B^{*}\right)$ and $\mathscr{R}\left[\begin{array}{l}A \\ B\end{array}\right] \subseteq \mathscr{R}\left[\begin{array}{l}A A^{*} \\ B B^{*}\end{array}\right]$.

$\langle 15\rangle\left\{A^{(1,2,4)}\right\} \cap\left\{B^{(1,3)}\right\} \neq \emptyset \Leftrightarrow \mathscr{R}\left(B A^{*}\right)=\mathscr{R}(B)$ and $B^{*} A A^{*}=B^{*} B A^{*}$.

$\langle 16\rangle\left\{A^{(1,2,4)}\right\} \cap\left\{B^{(1,2)}\right\} \neq \emptyset \Leftrightarrow \mathscr{R}\left(A B^{*}\right)=\mathscr{R}(A), \mathscr{R}\left(B A^{*}\right)=\mathscr{R}(B)$, and $\mathscr{R}\left[\begin{array}{c}A B^{*} \\ B^{*}\end{array}\right] \subseteq \mathscr{R}\left[\begin{array}{c}A A^{*} \\ B^{*}\end{array}\right]$.

$\langle 17\rangle\left\{A^{(1,2,4)}\right\} \cap\left\{B^{(1)}\right\} \neq \emptyset \Leftrightarrow \mathscr{R}\left(B A^{*}\right)=\mathscr{R}(B)$ and $\mathscr{R}\left[\begin{array}{c}A B^{*} \\ B^{*}\end{array}\right] \subseteq \mathscr{R}\left[\begin{array}{c}A A^{*} \\ B^{*}\end{array}\right]$.

$\langle 18\rangle\left\{A^{(1,4)}\right\} \cap\left\{B^{(1,4)}\right\} \neq \emptyset \Leftrightarrow \mathscr{R}\left[\begin{array}{l}A \\ B\end{array}\right] \subseteq \mathscr{R}\left[\begin{array}{l}A A^{*} \\ B B^{*}\end{array}\right]$.

$\langle 19\rangle\left\{A^{(1,4)}\right\} \cap\left\{B^{(1,3)}\right\} \neq \emptyset \Leftrightarrow B^{*} A A^{*}=B^{*} B A^{*}$.

$\langle 20\rangle\left\{A^{(1,4)}\right\} \cap\left\{B^{(1,2)}\right\} \neq \emptyset \Leftrightarrow \mathscr{R}\left(A B^{*}\right)=\mathscr{R}(A)$ and $\mathscr{R}\left[\begin{array}{c}A B^{*} \\ B^{*}\end{array}\right] \subseteq \mathscr{R}\left[\begin{array}{c}A A^{*} \\ B^{*}\end{array}\right]$. 
$\langle 21\rangle\left\{A^{(1,4)}\right\} \cap\left\{B^{(1)}\right\} \neq \emptyset \Leftrightarrow \mathscr{R}\left[\begin{array}{c}A B^{*} \\ B^{*}\end{array}\right] \subseteq \mathscr{R}\left[\begin{array}{c}A A^{*} \\ B^{*}\end{array}\right]$.

$\langle 22\rangle\left\{A^{(1,2)}\right\} \cap\left\{B^{(1,2)}\right\} \neq \emptyset \Leftrightarrow r(A-B)=r\left[\begin{array}{l}A \\ B\end{array}\right]+r[A, B]-r(A)-r(B)$ and $r(A)=r(B)$.

$\langle 23\rangle\left\{A^{(1,2)}\right\} \cap\left\{B^{(1)}\right\} \neq \emptyset \Leftrightarrow r(A-B)=r\left[\begin{array}{l}A \\ B\end{array}\right]+r[A, B]-r(A)-r(B)$ and $r(A) \geq r(B)$.

$\langle 24\rangle\left\{A^{(1)}\right\} \cap\left\{B^{(1)}\right\} \neq \emptyset \Leftrightarrow r(A-B)=r\left[\begin{array}{l}A \\ B\end{array}\right]+r[A, B]-r(A)-r(B)$.

Lemma 7.2. Given $A, B \in \mathbb{C}^{m \times n}$, we have the following results:

$\langle 1 \mathrm{a}\rangle\left\{A^{(1,3,4)}\right\} \subseteq\left\{B^{(1,3,4)}\right\} \Leftrightarrow B^{*} A=B^{*} B$ and $A B^{*}=B B^{*}$.

$\langle 1 \mathrm{~b}\rangle\left\{A^{(1,3,4)}\right\} \supseteq\left\{B^{(1,3,4)}\right\} \Leftrightarrow A^{*} A=A^{*} B$ and $A A^{*}=B A^{*}$.

$\langle 1 \mathrm{c}\rangle\left\{A^{(1,3,4)}\right\}=\left\{B^{(1,3,4)}\right\} \Leftrightarrow A=B$.

$\langle 2 \mathrm{a}\rangle\left\{A^{(1,3,4)}\right\} \subseteq\left\{B^{(1,2,4)}\right\} \Leftrightarrow\{r(A)=m$ and $A=B\}$ or $\left\{r(A)=r(B)=n\right.$ and $\left.A^{*} A=A^{*} B\right\}$.

$\langle 2 \mathrm{~b}\rangle\left\{A^{(1,3,4)}\right\} \supseteq\left\{B^{(1,2,4)}\right\} \Leftrightarrow A=0$ or $\left\{r(B)=m, A^{*} A=A^{*} B\right.$, and $\left.A A^{*}=B A^{*}\right\}$.

$\langle 2 \mathrm{c}\rangle\left\{A^{(1,3,4)}\right\}=\left\{B^{(1,2,4)}\right\} \Leftrightarrow r(A)=m$ and $A=B$.

$\langle 3 \mathrm{a}\rangle\left\{A^{(1,3,4)}\right\} \subseteq\left\{B^{(1,4)}\right\} \Leftrightarrow\left\{\mathscr{R}\left(A^{*}\right) \supseteq \mathscr{R}\left(B^{*}\right)\right.$ and $\left.A B^{*}=B B^{*}\right\}$ or $\left\{r(A)=n\right.$ and $\left.A^{*} A B^{*}=A^{*} B B^{*}\right\}$.

$\langle 3 \mathrm{~b}\rangle\left\{A^{(1,3,4)}\right\} \supseteq\left\{B^{(1,4)}\right\} \Leftrightarrow A=0$ or $\left\{r(B)=m, A^{*} A=A^{*} B\right.$, and $\left.A A^{*}=B A^{*}\right\}$.

$\langle 3 \mathrm{c}\rangle\left\{A^{(1,3,4)}\right\}=\left\{B^{(1,4)}\right\} \Leftrightarrow A=B=0$ or $\{r(A)=m$ and $A=B\}$.

$\langle 4 \mathrm{a}\rangle\left\{A^{(1,3,4)}\right\} \subseteq\left\{B^{(1,2)}\right\} \Leftrightarrow\left\{r(A)=r(B)=m\right.$ and $\left.A A^{*}=B A^{*}\right\}$ or $\left\{r(A)=r(B)=n\right.$ and $\left.A^{*} A=A^{*} B\right\}$.

$\langle 4 \mathrm{~b}\rangle\left\{A^{(1,3,4)}\right\} \supseteq\left\{B^{(1,2)}\right\} \Leftrightarrow A=0$ or $\left\{r(B)=m=n, A^{*} A=A^{*} B\right.$, and $\left.A A^{*}=B A^{*}\right\}$.

$\langle 4 \mathrm{c}\rangle\left\{A^{(1,3,4)}\right\}=\left\{B^{(1,2)}\right\} \Leftrightarrow r(A)=m=n$ and $A=B$.

$\langle 5 \mathrm{a}\rangle\left\{A^{(1,3,4)}\right\} \subseteq\left\{B^{(1)}\right\} \Leftrightarrow \mathscr{R}\left[\begin{array}{l}A A^{*} \\ B A^{*}\end{array}\right] \supseteq \mathscr{R}\left[\begin{array}{l}B \\ B\end{array}\right]$ or $\mathscr{R}\left[\begin{array}{l}A^{*} A \\ B^{*} A\end{array}\right] \supseteq \mathscr{R}\left[\begin{array}{l}B^{*} \\ B^{*}\end{array}\right]$.

$\langle 5 \mathrm{~b}\rangle\left\{A^{(1,3,4)}\right\} \supseteq\left\{B^{(1)}\right\} \Leftrightarrow A=0$ or $\left\{r(B)=m=n, A^{*} A=A^{*} B\right.$, and $\left.A A^{*}=B A^{*}\right\}$.

$\langle 5 c\rangle\left\{A^{(1,3,4)}\right\}=\left\{B^{(1)}\right\} \Leftrightarrow A=B=0$ or $\{r(A)=m=n$ and $A=B\}$.

$\langle 6\rangle\left\{A^{(1,2,4)}\right\} \subseteq\left\{B^{(1,2,4)}\right\} \Leftrightarrow\left\{A^{(1,2,4)}\right\} \supseteq\left\{B^{(1,2,4)}\right\} \Leftrightarrow\left\{A^{(1,2,4)}\right\}=\left\{B^{(1,2,4)}\right\} \Leftrightarrow A=B$.

$\langle 7 \mathrm{a}\rangle\left\{A^{(1,2,4)}\right\} \subseteq\left\{B^{(1,2,3)}\right\} \Leftrightarrow A=B=0$ or $\left\{r(A)=r(B)=m\right.$ and $\left.A A^{*}=B A^{*}\right\}$.

$\langle 7 \mathrm{~b}\rangle\left\{A^{(1,2,4)}\right\} \supseteq\left\{B^{(1,2,3)}\right\} \Leftrightarrow A=B=0$ or $\left\{r(A)=r(B)=n\right.$ and $\left.B^{*} A=B^{*} B\right\}$.

$\langle 7 \mathrm{c}\rangle\left\{A^{(1,2,4)}\right\}=\left\{B^{(1,2,3)}\right\} \Leftrightarrow A=B=0$ or $\{r(A)=m=n$ and $A=B\}$.

$\langle 8 \mathrm{a}\rangle\left\{A^{(1,2,4)}\right\} \subseteq\left\{B^{(1,4)}\right\} \Leftrightarrow \mathscr{R}\left(A^{*}\right) \supseteq \mathscr{R}\left(B^{*}\right)$ and $A B^{*}=B B^{*}$.

$\langle 8 \mathrm{~b}\rangle\left\{A^{(1,2,4)}\right\} \supseteq\left\{B^{(1,4)}\right\} \Leftrightarrow r(A)=\min \{m, n\}$ and $A=B$.

$\langle 8 \mathrm{c}\rangle\left\{A^{(1,2,4)}\right\}=\left\{B^{(1,4)}\right\} \Leftrightarrow r(A)=\min \{m, n\}$ and $A=B$.

$\langle 9 \mathrm{a}\rangle\left\{A^{(1,2,4)}\right\} \subseteq\left\{B^{(1,3)}\right\} \Leftrightarrow B=0$ or $\left\{r(A)=m\right.$ and $\left.B^{*} A A^{*}=B^{*} B A^{*}\right\}$.

$\langle 9 \mathrm{~b}\rangle\left\{A^{(1,2,4)}\right\} \supseteq\left\{B^{(1,3)}\right\} \Leftrightarrow r(A)=r(B)=n$ and $B^{*} A=B^{*} B$.

$\langle 9 \mathrm{c}\rangle\left\{A^{(1,2,4)}\right\}=\left\{B^{(1,3)}\right\} \Leftrightarrow r(A)=m=n$ and $A=B$.

$\langle 10 \mathrm{a}\rangle\left\{A^{(1,2,4)}\right\} \subseteq\left\{B^{(1,2)}\right\} \Leftrightarrow \mathscr{R}(A)=\mathscr{R}(B)$ and $A A^{*}=B A^{*}$.

$\langle 10 \mathrm{~b}\rangle\left\{A^{(1,2,4)}\right\} \supseteq\left\{B^{(1,2)}\right\} \Leftrightarrow A=B=0$ or $\{r(A)=n$ and $A=B\}$.

$\langle 10 \mathrm{c}\rangle\left\{A^{(1,2,4)}\right\}=\left\{B^{(1,2)}\right\} \Leftrightarrow A=B=0$ or $\{r(A)=n$ and $A=B\}$. 
$\langle 11 \mathrm{a}\rangle\left\{A^{(1,2,4)}\right\} \subseteq\left\{B^{(1)}\right\} \Leftrightarrow \mathscr{R}\left[\begin{array}{l}A A^{*} \\ B A^{*}\end{array}\right] \supseteq \mathscr{R}\left[\begin{array}{l}B \\ B\end{array}\right]$.

$\langle 1 \mathrm{~b}\rangle\left\{A^{(1,2,4)}\right\} \supseteq\left\{B^{(1)}\right\} \Leftrightarrow r(A)=n$ and $A=B$.

$\langle 11 \mathrm{c}\rangle\left\{A^{(1,2,4)}\right\}=\left\{B^{(1)}\right\} \Leftrightarrow r(A)=n$ and $A=B$.

$\langle 12 \mathrm{a}\rangle\left\{A^{(1,4)}\right\} \subseteq\left\{B^{(1,4)}\right\} \Leftrightarrow \mathscr{R}\left(A^{*}\right) \supseteq \mathscr{R}\left(B^{*}\right)$ and $A B^{*}=B B^{*}$.

$\langle 12 \mathrm{~b}\rangle\left\{A^{(1,4)}\right\} \supseteq\left\{B^{(1,4)}\right\} \Leftrightarrow \mathscr{R}\left(A^{*}\right) \subseteq \mathscr{R}\left(B^{*}\right)$ and $A A^{*}=B A^{*}$.

$\langle 12 \mathrm{c}\rangle\left\{A^{(1,4)}\right\}=\left\{B^{(1,4)}\right\} \Leftrightarrow A=B$.

$\langle 13 \mathrm{a}\rangle\left\{A^{(1,4)}\right\} \subseteq\left\{B^{(1,3)}\right\} \Leftrightarrow B=0$ or $\left\{r(A)=m\right.$ and $\left.B^{*} A A^{*}=B^{*} B A^{*}\right\}$.

$\langle 13 \mathrm{~b}\rangle\left\{A^{(1,4)}\right\} \supseteq\left\{B^{(1,3)}\right\} \Leftrightarrow A=0$ or $\left\{r(B)=n\right.$ and $\left.B^{*} A A^{*}=B^{*} B A^{*}\right\}$.

$\langle 13 \mathrm{c}\rangle\left\{A^{(1,4)}\right\}=\left\{B^{(1,3)}\right\} \Leftrightarrow A=B=0$ or $\left\{r(A)=m, r(B)=n\right.$, and $\left.B^{*} A A^{*}=B^{*} B A^{*}\right\}$.

$\langle 14 \mathrm{a}\rangle\left\{A^{(1,4)}\right\} \subseteq\left\{B^{(1,2)}\right\} \Leftrightarrow r(A)=r(B)=\min \{m, \quad n\}$ and $A A^{*}=B A^{*}$.

$\langle 14 \mathrm{~b}\rangle\left\{A^{(1,4)}\right\} \supseteq\left\{B^{(1,2)}\right\} \Leftrightarrow A=0$ or $\left\{r(B)=n\right.$ and $\left.A A^{*}=B A^{*}\right\}$.

$\langle 14 \mathrm{c}\rangle\left\{A^{(1,4)}\right\}=\left\{B^{(1,2)}\right\} \Leftrightarrow r(A)=r(B)=n$ and $A=B$.

$\langle 15 \mathrm{a}\rangle\left\{A^{(1,4)}\right\} \subseteq\left\{B^{(1)}\right\} \Leftrightarrow \mathscr{R}\left[\begin{array}{l}A A^{*} \\ B A^{*}\end{array}\right] \supseteq \mathscr{R}\left[\begin{array}{l}B \\ B\end{array}\right]$.

$\langle 15 \mathrm{~b}\rangle\left\{A^{(1,4)}\right\} \supseteq\left\{B^{(1)}\right\} \Leftrightarrow A=0$ or $\left\{r(B)=n\right.$ and $\left.A A^{*}=B A^{*}\right\}$.

$\langle 15 \mathrm{c}\rangle\left\{A^{(1,4)}\right\}=\left\{B^{(1)}\right\} \Leftrightarrow A=B=0$ or $\{r(A)=n$ and $A=B\}$.

$\langle 16\rangle\left\{A^{(1,2)}\right\} \subseteq\left\{B^{(1,2)}\right\} \Leftrightarrow\left\{A^{(1,2)}\right\} \supseteq\left\{B^{(1,2)}\right\} \Leftrightarrow\left\{A^{(1,2)}\right\}=\left\{B^{(1,2)}\right\} \Leftrightarrow A=B$.

$\langle 17 \mathrm{a}\rangle\left\{A^{(1,2)}\right\} \subseteq\left\{B^{(1)}\right\} \Leftrightarrow r(A-B)=r(A)-r(B)$.

$\langle 17 \mathrm{~b}\rangle\left\{A^{(1,2)}\right\} \supseteq\left\{B^{(1)}\right\} \Leftrightarrow r(A)=\min \{m, n\}$ and $A=B$.

$\langle 17 \mathrm{c}\rangle\left\{A^{(1,2)}\right\}=\left\{B^{(1)}\right\} \Leftrightarrow r(A)=\min \{m, n\}$ and $A=B$.

$\langle 18 \mathrm{a}\rangle\left\{A^{(1)}\right\} \subseteq\left\{B^{(1)}\right\} \Leftrightarrow r(A-B)=r(A)-r(B)$.

$\langle 18 \mathrm{~b}\rangle\left\{A^{(1)}\right\} \supseteq\left\{B^{(1)}\right\} \Leftrightarrow r(B-A)=r(B)-r(A)$.

$\langle 18 \mathrm{c}\rangle\left\{A^{(1)}\right\}=\left\{B^{(1)}\right\} \Leftrightarrow A=B$.

The results in Lemmas 7.1 and 7.2 can be simplified further for specified matrices $A$ and $B$. For example, applying Lemmas 7.1 and 7.2 to two orthogonal projectors of the same size yields the following consequences.

Corollary 7.3. Given two orthogonal projectors $A, B \in \mathbb{C}^{m \times m}$, namely, $A^{2}=A=A^{*}$ and $B^{2}=B=B^{*}$, we have the following 23 results:

$\langle 1\rangle A \in\left\{B^{(1,3,4)}\right\} \Leftrightarrow \mathscr{R}(A) \supseteq \mathscr{R}(B)$.

$\langle 2\rangle A \in\left\{B^{(1,2,4)}\right\} \Leftrightarrow A=B$.

$\langle 3\rangle A \in\left\{B^{(1,4)}\right\} \Leftrightarrow \mathscr{R}(A) \supseteq \mathscr{R}(B)$.

$\langle 4\rangle A \in\left\{B^{(1,2)}\right\} \Leftrightarrow r(A)=r(B)$ and $A=A B A$.

$\langle 5\rangle A \in\left\{B^{(1)}\right\} \Leftrightarrow r(A-A B A)=r(A)-r(B)$.

$\langle 6\rangle\left\{A^{(1,3,4)}\right\} \cap\left\{B^{(1,3,4)}\right\} \neq \emptyset$ holds.

$\langle 7\rangle\left\{A^{(1,3,4)}\right\} \cap\left\{B^{(1,2,4)}\right\} \neq \emptyset \Leftrightarrow \mathscr{R}(A) \subseteq \mathscr{R}(B)$.

$\langle 8\rangle\left\{A^{(1,3,4)}\right\} \cap\left\{B^{(1,4)}\right\} \neq \emptyset$ holds.

$\langle 9\rangle\left\{A^{(1,3,4)}\right\} \cap\left\{B^{(1,2)}\right\} \neq \emptyset \Leftrightarrow A=A B A$.

$\langle 10\rangle\left\{A^{(1,3,4)}\right\} \cap\left\{B^{(1)}\right\} \neq \emptyset$ holds. 
$\langle 11\rangle\left\{A^{(1,2,4)}\right\} \cap\left\{B^{(1,2,4)}\right\} \neq \emptyset \Leftrightarrow A=B$.

$\langle 12\rangle\left\{A^{(1,2,4)}\right\} \cap\left\{B^{(1,2,3)}\right\} \neq \emptyset \Leftrightarrow r(A B)=r(B A)=r(A)=r(B)$.

$\langle 13\rangle\left\{A^{(1,2,4)}\right\} \cap\left\{B^{(1,4)}\right\} \neq \emptyset \Leftrightarrow \mathscr{R}(A) \supseteq \mathscr{R}(B)$.

$\langle 14\rangle\left\{A^{(1,2,4)}\right\} \cap\left\{B^{(1,3)}\right\} \neq \emptyset \Leftrightarrow \mathscr{R}(B A)=\mathscr{R}(B)$.

$\langle 15\rangle\left\{A^{(1,2,4)}\right\} \cap\left\{B^{(1,2)}\right\} \neq \emptyset \Leftrightarrow \mathscr{R}(A B)=\mathscr{R}(A)$ and $\mathscr{R}(B A)=\mathscr{R}(B)$.

$\langle 16\rangle\left\{A^{(1,2,4)}\right\} \cap\left\{B^{(1)}\right\} \neq \emptyset \Leftrightarrow \mathscr{R}(B A)=\mathscr{R}(B)$.

$\langle 17\rangle\left\{A^{(1,4)}\right\} \cap\left\{B^{(1,4)}\right\} \neq \emptyset$ holds.

$\langle 18\rangle\left\{A^{(1,4)}\right\} \cap\left\{B^{(1,3)}\right\} \neq \emptyset$ holds.

$\langle 19\rangle\left\{A^{(1,4)}\right\} \cap\left\{B^{(1,2)}\right\} \neq \emptyset \Leftrightarrow \mathscr{R}(A B)=\mathscr{R}(A)$.

$\langle 20\rangle\left\{A^{(1,4)}\right\} \cap\left\{B^{(1)}\right\} \neq \emptyset$ holds.

$\langle 21\rangle\left\{A^{(1,2)}\right\} \cap\left\{B^{(1,2)}\right\} \neq \emptyset \Leftrightarrow r(A-B)=2 r[A, B]-2 r(A)$ and $r(A)=r(B)$.

$\langle 22\rangle\left\{A^{(1,2)}\right\} \cap\left\{B^{(1)}\right\} \neq \emptyset \Leftrightarrow r(A-B)=2 r[A, B]-r(A)-r(B)$ and $r(A) \geq r(B)$.

$\langle 23\rangle\left\{A^{(1)}\right\} \cap\left\{B^{(1)}\right\} \neq \emptyset \Leftrightarrow r(A-B)=2 r[A, B]-r(A)-r(B)$.

Corollary 7.4. Given two orthogonal projectors $A, B \in \mathbb{C}^{m \times m}$, we have the following results:

$\langle 1 \mathrm{a}\rangle\left\{A^{(1,3,4)}\right\} \subseteq\left\{B^{(1,3,4)}\right\} \Leftrightarrow \mathscr{R}(A) \supseteq \mathscr{R}(B)$.

$\langle 1 \mathrm{~b}\rangle\left\{A^{(1,3,4)}\right\} \supseteq\left\{B^{(1,3,4)}\right\} \Leftrightarrow \mathscr{R}(A) \subseteq \mathscr{R}(B)$.

$\langle 1 \mathrm{c}\rangle\left\{A^{(1,3,4)}\right\}=\left\{B^{(1,3,4)}\right\} \Leftrightarrow A=B$.

$\langle 2 \mathrm{a}\rangle\left\{A^{(1,3,4)}\right\} \subseteq\left\{B^{(1,2,4)}\right\} \Leftrightarrow A=B=I_{m}$.

$\langle 2 \mathrm{~b}\rangle\left\{A^{(1,3,4)}\right\} \supseteq\left\{B^{(1,2,4)}\right\} \Leftrightarrow A=0$ or $B=I_{m}$.

$\langle 2 \mathrm{c}\rangle\left\{A^{(1,3,4)}\right\}=\left\{B^{(1,2,4)}\right\} \Leftrightarrow A=B=I_{m}$.

$\langle 3 \mathrm{a}\rangle\left\{A^{(1,3,4)}\right\} \subseteq\left\{B^{(1,4)}\right\} \Leftrightarrow \mathscr{R}(A) \supseteq \mathscr{R}(B)$.

$\langle 3 \mathrm{~b}\rangle\left\{A^{(1,3,4)}\right\} \supseteq\left\{B^{(1,4)}\right\} \Leftrightarrow A=0$ or $B=I_{m}$.

$\langle 3 \mathrm{c}\rangle\left\{A^{(1,3,4)}\right\}=\left\{B^{(1,4)}\right\} \Leftrightarrow A=B=0$ or $A=B=I_{m}$.

$\langle 4 \mathrm{a}\rangle\left\{A^{(1,3,4)}\right\} \supseteq\left\{B^{(1,2)}\right\} \Leftrightarrow A=0$ or $B=I_{m}$.

$\langle 4 \mathrm{~b}\rangle\left\{A^{(1,3,4)}\right\} \subseteq\left\{B^{(1,2)}\right\} \Leftrightarrow\left\{A^{(1,3,4)}\right\}=\left\{B^{(1,2)}\right\} \Leftrightarrow A=B=I_{m}$.

$\langle 5 \mathrm{a}\rangle\left\{A^{(1,3,4)}\right\} \subseteq\left\{B^{(1)}\right\} \Leftrightarrow \mathscr{R}(A) \supseteq \mathscr{R}(B)$.

$\langle 5 \mathrm{~b}\rangle\left\{A^{(1,3,4)}\right\} \supseteq\left\{B^{(1)}\right\} \Leftrightarrow A=0$ or $B=I_{m}$.

$\langle 5 \mathrm{c}\rangle\left\{A^{(1,3,4)}\right\}=\left\{B^{(1)}\right\} \Leftrightarrow A=B=0$ or $A=B=I_{m}$.

$\langle 6\rangle\left\{A^{(1,2,4)}\right\} \subseteq\left\{B^{(1,2,4)}\right\} \Leftrightarrow\left\{A^{(1,2,4)}\right\} \supseteq\left\{B^{(1,2,4)}\right\} \Leftrightarrow\left\{A^{(1,2,4)}\right\}=\left\{B^{(1,2,4)}\right\} \Leftrightarrow A=B$.

$\langle 7\rangle\left\{A^{(1,2,4)}\right\} \subseteq\left\{B^{(1,2,3)}\right\} \Leftrightarrow\left\{A^{(1,2,4)}\right\} \supseteq\left\{B^{(1,2,3)}\right\} \Leftrightarrow\left\{A^{(1,2,4)}\right\}=\left\{B^{(1,2,3)}\right\} \Leftrightarrow A=B=0$ or $A=B=I_{m}$.

$\langle 8 \mathrm{a}\rangle\left\{A^{(1,2,4)}\right\} \subseteq\left\{B^{(1,4)}\right\} \Leftrightarrow \mathscr{R}(A) \supseteq \mathscr{R}(B)$.

$\langle 8 \mathrm{~b}\rangle\left\{A^{(1,2,4)}\right\} \supseteq\left\{B^{(1,4)}\right\} \Leftrightarrow\left\{A^{(1,2,4)}\right\}=\left\{B^{(1,4)}\right\} \Leftrightarrow A=B=I_{m}$.

$\langle 9 \mathrm{a}\rangle\left\{A^{(1,2,4)}\right\} \subseteq\left\{B^{(1,3)}\right\} \Leftrightarrow B=0$ or $A=I_{m}$.

$\langle 9 \mathrm{~b}\rangle\left\{A^{(1,2,4)}\right\} \supseteq\left\{B^{(1,3)}\right\} \Leftrightarrow\left\{A^{(1,2,4)}\right\}=\left\{B^{(1,3)}\right\} \Leftrightarrow A=B=I_{m}$.

$\langle 10 \mathrm{a}\rangle\left\{A^{(1,2,4)}\right\} \subseteq\left\{B^{(1,2)}\right\} \Leftrightarrow A=B$.

$\langle 10 \mathrm{~b}\rangle\left\{A^{(1,2,4)}\right\} \supseteq\left\{B^{(1,2)}\right\} \Leftrightarrow\left\{A^{(1,2,4)}\right\}=\left\{B^{(1,2)}\right\} \Leftrightarrow A=B=0$ or $A=B=I_{m}$.

$\langle 11 \mathrm{a}\rangle\left\{A^{(1,2,4)}\right\} \subseteq\left\{B^{(1)}\right\} \Leftrightarrow \mathscr{R}(A) \supseteq \mathscr{R}(B)$. 
$\langle 11 \mathrm{~b}\rangle\left\{A^{(1,2,4)}\right\} \supseteq\left\{B^{(1)}\right\} \Leftrightarrow\left\{A^{(1,2,4)}\right\}=\left\{B^{(1)}\right\} \Leftrightarrow A=B=I_{m}$.

$\langle 12 \mathrm{a}\rangle\left\{A^{(1,4)}\right\} \subseteq\left\{B^{(1,4)}\right\} \Leftrightarrow \mathscr{R}(A) \supseteq \mathscr{R}(B)$.

$\langle 12 \mathrm{~b}\rangle\left\{A^{(1,4)}\right\} \supseteq\left\{B^{(1,4)}\right\} \Leftrightarrow \mathscr{R}(A) \subseteq \mathscr{R}(B)$.

$\langle 12 \mathrm{c}\rangle\left\{A^{(1,4)}\right\}=\left\{B^{(1,4)}\right\} \Leftrightarrow A=B$.

$\langle 13 \mathrm{a}\rangle\left\{A^{(1,4)}\right\} \subseteq\left\{B^{(1,3)}\right\} \Leftrightarrow B=0$ or $A=I_{m}$.

$\langle 13 \mathrm{~b}\rangle\left\{A^{(1,4)}\right\} \supseteq\left\{B^{(1,3)}\right\} \Leftrightarrow A=0$ or $B=I_{m}$.

$\langle 13 \mathrm{c}\rangle\left\{A^{(1,4)}\right\}=\left\{B^{(1,3)}\right\} \Leftrightarrow A=B=0$ or $A=B=I_{m}$.

$\langle 14 \mathrm{a}\rangle\left\{A^{(1,4)}\right\} \supseteq\left\{B^{(1,2)}\right\} \Leftrightarrow A=0$ or $B=I_{m}$.

$\langle 14 \mathrm{~b}\rangle\left\{A^{(1,4)}\right\} \subseteq\left\{B^{(1,2)}\right\} \Leftrightarrow\left\{A^{(1,4)}\right\}=\left\{B^{(1,2)}\right\} \Leftrightarrow A=B=I_{m}$.

$\langle 15 \mathrm{a}\rangle\left\{A^{(1,4)}\right\} \subseteq\left\{B^{(1)}\right\} \Leftrightarrow \mathscr{R}(A) \supseteq \mathscr{R}(B)$.

$\langle 15 \mathrm{~b}\rangle\left\{A^{(1,4)}\right\} \supseteq\left\{B^{(1)}\right\} \Leftrightarrow A=0$ or $B=I_{m}$.

$\langle 15 \mathrm{c}\rangle\left\{A^{(1,4)}\right\}=\left\{B^{(1)}\right\} \Leftrightarrow A=B=0$ or $A=B=I_{m}$.

$\langle 16\rangle\left\{A^{(1,2)}\right\} \subseteq\left\{B^{(1,2)}\right\} \Leftrightarrow\left\{A^{(1,2)}\right\} \supseteq\left\{B^{(1,2)}\right\} \Leftrightarrow\left\{A^{(1,2)}\right\}=\left\{B^{(1,2)}\right\} \Leftrightarrow A=B$.

$\langle 17 \mathrm{a}\rangle\left\{A^{(1,2)}\right\} \subseteq\left\{B^{(1)}\right\} \Leftrightarrow r(A-B)=r(A)-r(B)$.

$\langle 17 \mathrm{~b}\rangle\left\{A^{(1,2)}\right\} \supseteq\left\{B^{(1)}\right\} \Leftrightarrow\left\{A^{(1,2)}\right\}=\left\{B^{(1)}\right\} \Leftrightarrow A=B=I_{m}$.

$\langle 18 \mathrm{a}\rangle\left\{A^{(1)}\right\} \subseteq\left\{B^{(1)}\right\} \Leftrightarrow r(A-B)=r(A)-r(B)$.

$\langle 18 \mathrm{~b}\rangle\left\{A^{(1)}\right\} \supseteq\left\{B^{(1)}\right\} \Leftrightarrow r(B-A)=r(B)-r(A)$.

$\langle 18 \mathrm{c}\rangle\left\{A^{(1)}\right\}=\left\{B^{(1)}\right\} \Leftrightarrow A=B$.

The preceding results show that much work is involved in the establishments of the simplest matrix equalities for two generalized inverses, so that the approaches on general matrix equalities for generalized inverses will become quite complicated.

\section{Ranks of matrix expressions composed by two generalized inverses}

In order to characterize the set inclusions in (1.9), we first need to know some fundamental properties of the products of generalized inverses. In this section, we present a variety of known results concerning the ranks and uniqueness of the product $B^{\left(s_{2}, \ldots, t_{2}\right)} A^{\left(s_{1}, \ldots, t_{1}\right)}$ and their variations, which will be used to characterize (1.7) under assumptions.

It is obvious that (1.7) includes a large variety of situations with symmetric pattern. So the following lemma can be used to characterize many set inclusions by symmetry.

Lemma 8.1. Let $A \in \mathbb{C}^{m \times n}$ and $B \in \mathbb{C}^{n \times p}$. Then the following 64 matrix set identities

$$
\left\{\left(B^{\left(s_{2}, \ldots, t_{2}\right)} A^{\left(s_{1}, \ldots, t_{1}\right)}\right)^{*}\right\}=\left\{\left(A^{*}\right)^{(i, \ldots, j)}\left(B^{*}\right)^{(g, \ldots, h)}\right\}
$$

hold according to Lemma 3.1(c) for the eight commonly-used types of generalized inverses of $A$ and $B$.

Lemma 8.2 ( [73]). Let $A \in \mathbb{C}^{m \times n}$ and $B \in \mathbb{C}^{n \times p}$ be given. Then the product $B^{\dagger} A^{\dagger}$ can be written as

$$
B^{\dagger} A^{\dagger}=-\left[B^{*}, 0\right]\left[\begin{array}{cc}
0 & A^{*} A A^{*} \\
B^{*} B B^{*} & B^{*} A^{*}
\end{array}\right]^{\dagger}\left[\begin{array}{c}
A^{*} \\
0
\end{array}\right] \triangleq-P J^{\dagger} Q
$$

where the block matrices $P, J$, and $Q$ satisfy $r(J)=r(A)+r(B), \mathscr{R}(Q) \subseteq \mathscr{R}(J)$, and $\mathscr{R}\left(P^{*}\right) \subseteq \mathscr{R}\left(J^{*}\right)$. 
Note that the rank of the product $B^{\left(s_{2}, \ldots, t_{2}\right)} A^{\left(s_{1}, \ldots, t_{1}\right)}$ may vary with respect to the choice of $A^{\left(s_{1}, \ldots, t_{1}\right)}$ and $B^{\left(s_{2}, \ldots, t_{2}\right)}$ (the variable matrices in the analytical expressions of $A^{\left(s_{1}, \ldots, t_{1}\right)}$ and $B^{\left(s_{2}, \ldots, t_{2}\right)}$ ). Also note from Lemma 3.2 that the ranks of the 64 products $B^{\left(s_{2}, \ldots, t_{2}\right)} A^{\left(s_{1}, \ldots, t_{1}\right)}$ are involved in the set inclusions for the $\{1,2\}-,\{1,2,3\}-$, and $\{1,2,4\}$-generalized inverses of $A B$. Thus it is imperative to determine the maximum and minimum ranks of $B^{\left(s_{2}, \ldots, t_{2}\right)} A^{\left(s_{1}, \ldots, t_{1}\right)}$ with respect to the choice of the generalized inverses. In the past several decades, a great achievement in linear algebra is the sufficient development of the matrix rank theory. Thousands of analytical formulas for calculating (maximum and minimum) ranks of matrix expressions have been established, and numerous consequences and applications of these matrix rank formulas have been obtained. In recent two papers [86, 87], the present author provided a comprehensive study of the rank problems of matrix expressions composed a pair of matrices and their generalized inverses, including the following analytical formulas for calculating the maximum and minimum ranks of $B^{\left(s_{2}, \ldots, t_{2}\right)} A^{\left(s_{1}, \ldots, t_{1}\right)}$ with respect to the choice of $A^{\left(s_{1}, \ldots, t_{1}\right)}$ and $B^{\left(s_{2}, \ldots, t_{2}\right)}$.

Lemma 8.3 ( [86]). Let $A \in \mathbb{C}^{m \times n}$ and $B \in \mathbb{C}^{n \times p}$ be given, and denote

$$
\begin{aligned}
& M=A B, \quad N=\left[A^{*}, B\right], \quad t=m+p+r(M)-r(A)-r(B), \\
& t_{1}=m+r(M)-r(A), \quad t_{2}=p+r(M)-r(B) .
\end{aligned}
$$

We also use the notation $u\left(B^{\left(s_{2}, \ldots, t_{2}\right)} A^{\left(s_{1}, \ldots, t_{1}\right)}\right)$ and $v\left(B^{\left(s_{2}, \ldots, t_{2}\right)} A^{\left(s_{1}, \ldots, t_{1}\right)}\right)$ to denote the maximum and minimum ranks of the product $B^{\left(s_{2}, \ldots, t_{2}\right)} A^{\left(s_{1}, \ldots, t_{1}\right)}$ with respect to the choice of $A^{\left(s_{1}, \ldots, t_{1}\right)}$ and $B^{\left(s_{2}, \ldots, t_{2}\right)}$, respectively. Then

$$
\begin{aligned}
& u\left(B^{\dagger} A^{(1,3,4)}\right)=\min \left\{r(B), t_{1}\right\}, \\
& u\left(B^{\dagger} A^{(1,2,4)}\right)=r(M) \text {, } \\
& u\left(B^{\dagger} A^{(1,2,3)}\right)=\min \{r(A), r(B)\}, \\
& u\left(B^{\dagger} A^{(1,4)}\right)=\min \left\{r(B), t_{1}\right\}, \\
& u\left(B^{\dagger} A^{(1,3)}\right)=\min \{m, r(B)\}, \\
& u\left(B^{\dagger} A^{(1,2)}\right)=\min \{r(A), r(B)\}, \\
& u\left(B^{\dagger} A^{(1)}\right)=\min \{m, r(B)\} \text {, } \\
& u\left(B^{(1,3,4)} A^{\dagger}\right)=\min \left\{r(A), t_{2}\right\}, \\
& u\left(B^{(1,3,4)} A^{(1,3,4)}\right)=\min \{m, n, p, t\}, \\
& u\left(B^{(1,3,4)} A^{(1,2,4)}\right)=\min \left\{r(A), t_{2}\right\} \text {, } \\
& u\left(B^{(1,3,4)} A^{(1,2,3)}\right)=\min \{p, r(A)\}, \\
& u\left(B^{(1,3,4)} A^{(1,4)}\right)=\min \{m, n, p, t\} \text {, } \\
& u\left(B^{(1,3,4)} A^{(1,3)}\right)=\min \{m, n, p\}, \\
& u\left(B^{(1,3,4)} A^{(1,2)}\right)=\min \{p, r(A)\}, \\
& u\left(B^{(1,3,4)} A^{(1)}\right)=\min \{m, n, p\}, \\
& u\left(B^{(1,2,4)} A^{\dagger}\right)=\min \{r(A), r(B)\}, \\
& u\left(B^{(1,2,4)} A^{(1,3,4)}\right)=\min \{m, r(B)\} \text {, } \\
& u\left(B^{(1,2,4)} A^{(1,2,4)}\right)=\min \{r(A), r(B)\}, \\
& u\left(B^{(1,2,4)} A^{(1,2,3)}\right)=\min \{r(A), r(B)\}, \\
& u\left(B^{(1,2,4)} A^{(1,4)}\right)=\min \{m, r(B)\}, \\
& u\left(B^{(1,2,4)} A^{(1,3)}\right)=\min \{m, r(B)\} \text {, } \\
& u\left(B^{(1,2,4)} A^{(1,2)}\right)=\min \{r(A), r(B)\}, \\
& u\left(B^{(1,2,4)} A^{(1)}\right)=\min \{m, r(B)\} \text {, } \\
& u\left(B^{(1,2,3)} A^{\dagger}\right)=r(M), \\
& u\left(B^{(1,2,3)} A^{(1,3,4)}\right)=\min \left\{r(B), t_{1}\right\}, \\
& u\left(B^{(1,2,3)} A^{(1,2,4)}\right)=r(M), \\
& u\left(B^{(1,2,3)} A^{(1,2,3)}\right)=\min \{r(A), r(B)\}, \\
& u\left(B^{(1,2,3)} A^{(1,4)}\right)=\min \left\{r(B), t_{1}\right\}, \\
& u\left(B^{(1,2,3)} A^{(1,3)}\right)=\min \{m, r(B)\} \text {, } \\
& u\left(B^{(1,2,3)} A^{(1,2)}\right)=\min \{r(A), r(B)\} \text {, } \\
& u\left(B^{(1,2,3)} A^{(1)}\right)=\min \{m, r(B)\} \text {, } \\
& u\left(B^{(1,4)} A^{\dagger}\right)=\min \{p, r(A)\} \text {, } \\
& u\left(B^{(1,4)} A^{(1,3,4)}\right)=\min \{m, n, p\}, \\
& u\left(B^{(1,4)} A^{(1,2,4)}\right)=\min \{p, r(A)\} \text {, } \\
& u\left(B^{(1,4)} A^{(1,2,3)}\right)=\min \{p, r(A)\}, \\
& u\left(B^{(1,4)} A^{(1,4)}\right)=\min \{m, n, p\}, \\
& u\left(B^{(1,4)} A^{(1,3)}\right)=\min \{m, n, p\},
\end{aligned}
$$

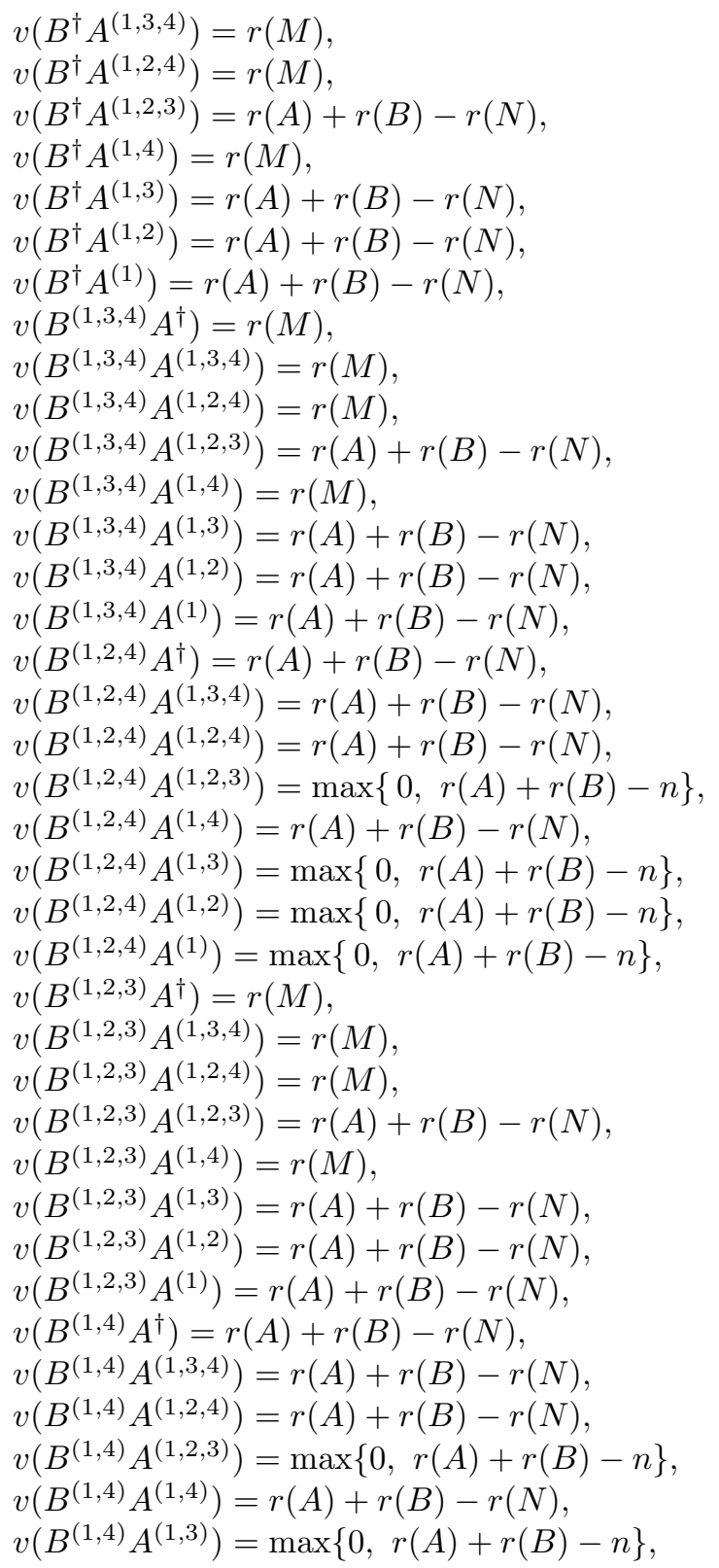


$u\left(B^{(1,4)} A^{(1,2)}\right)=\min \{p, r(A)\}$, $u\left(B^{(1,4)} A^{(1)}\right)=\min \{m, n, p\}$, $u\left(B^{(1,3)} A^{\dagger}\right)=\min \left\{r(A), t_{2}\right\}$, $u\left(B^{(1,3)} A^{(1,3,4)}\right)=\min \{m, n, p, t\}$, $u\left(B^{(1,3)} A^{(1,2,4)}\right)=\min \left\{r(A), t_{2}\right\}$, $u\left(B^{(1,3)} A^{(1,2,3)}\right)=\min \{p, r(A)\}$, $u\left(B^{(1,3)} A^{(1,4)}\right)=\min \{m, n, p, t\}$, $u\left(B^{(1,3)} A^{(1,3)}\right)=\min \{m, n, p\}$, $u\left(B^{(1,3)} A^{(1,2)}\right)=\min \{p, r(A)\}$, $u\left(B^{(1,3)} A^{(1)}\right)=\min \{m, n, p\}$, $u\left(B^{(1,2)} A^{\dagger}\right)=\min \{r(A), r(B)\}$, $u\left(B^{(1,2)} A^{(1,3,4)}\right)=\min \{m, r(B)\}$, $u\left(B^{(1,2)} A^{(1,2,4)}\right)=\min \{r(A), r(B)\}$, $u\left(B^{(1,2)} A^{(1,2,3)}\right)=\min \{r(A), r(B)\}$, $u\left(B^{(1,2)} A^{(1,4)}\right)=\min \{m, r(B)\}$, $u\left(B^{(1,2)} A^{(1,3)}\right)=\min \{m, r(B)\}$, $u\left(B^{(1,2)} A^{(1,2)}\right)=\min \{r(A), r(B)\}$, $u\left(B^{(1,2)} A^{(1)}\right)=\min \{m, r(B)\}$, $u\left(B^{(1)} A^{\dagger}\right)=\min \{p, r(A)\}$, $u\left(B^{(1)} A^{(1,3,4)}\right)=\min \{m, n, p\}$, $u\left(B^{(1)} A^{(1,2,4)}\right)=\min \{p, r(A)\}$, $u\left(B^{(1)} A^{(1,2,3)}\right)=\min \{p, r(A)\}$, $u\left(B^{(1)} A^{(1,4)}\right)=\min \{m, n, p\}$, $u\left(B^{(1)} A^{(1,3)}\right)=\min \{m, n, p\}$, $u\left(B^{(1)} A^{(1,2)}\right)=\min \{p, r(A)\}$, $u\left(B^{(1)} A^{(1)}\right)=\min \{m, n, p\}$,
$v\left(B^{(1,4)} A^{(1,2)}\right)=\max \{0, r(A)+r(B)-n\}$,
$v\left(B^{(1,4)} A^{(1)}\right)=\max \{0, r(A)+r(B)-n\}$,
$v\left(B^{(1,3)} A^{\dagger}\right)=r(M)$,
$v\left(B^{(1,3)} A^{(1,3,4)}\right)=r(M)$,
$v\left(B^{(1,3)} A^{(1,2,4)}\right)=r(M)$,
$v\left(B^{(1,3)} A^{(1,2,3)}\right)=r(A)+r(B)-r(N)$,
$v\left(B^{(1,3)} A^{(1,4)}\right)=r(M)$,
$v\left(B^{(1,3)} A^{(1,3)}\right)=r(A)+r(B)-r(N)$,
$v\left(B^{(1,3)} A^{(1,2)}\right)=r(A)+r(B)-r(N)$,
$v\left(B^{(1,3)} A^{(1)}\right)=r(A)+r(B)-r(N)$,
$v\left(B^{(1,2)} A^{\dagger}\right)=r(A)+r(B)-r(N)$,
$v\left(B^{(1,2)} A^{(1,3,4)}\right)=r(A)+r(B)-r(N)$,
$v\left(B^{(1,2)} A^{(1,2,4)}\right)=r(A)+r(B)-r(N)$,
$v\left(B^{(1,2)} A^{(1,2,3)}\right)=\max \{0, r(A)+r(B)-n\}$,
$v\left(B^{(1,2)} A^{(1,4)}\right)=r(A)+r(B)-r(N)$,
$v\left(B^{(1,2)} A^{(1,3)}\right)=\max \{0, r(A)+r(B)-n\}$,
$v\left(B^{(1,2)} A^{(1,2)}\right)=\max \{0, r(A)+r(B)-n\}$,
$v\left(B^{(1,2)} A^{(1)}\right)=\max \{0, r(A)+r(B)-n\}$,
$v\left(B^{(1)} A^{\dagger}\right)=r(A)+r(B)-r(N)$,
$v\left(B^{(1)} A^{(1,3,4)}\right)=r(A)+r(B)-r(N)$,
$v\left(B^{(1)} A^{(1,2,4)}\right)=r(A)+r(B)-r(N)$,
$v\left(B^{(1)} A^{(1,2,3)}\right)=\max \{0, r(A)+r(B)-n\}$,
$v\left(B^{(1)} A^{(1,4)}\right)=r(A)+r(B)-r(N)$,
$v\left(B^{(1)} A^{(1,3)}\right)=\max \{0, r(A)+r(B)-n\}$,
$v\left(B^{(1)} A^{(1,2)}\right)=\max \{0, r(A)+r(B)-n\}$
$v\left(B^{(1)} A^{(1)}\right)=\max \{0, r(A)+r(B)-n\}$.

Notice that the rank formulas in Lemma 8.3 are all given in simple and analytical forms. Thus, we can directly use them to describe algebraic performance of the products of generalized inverses in many situations. In particular, they can be used to characterize the rank invariance of $B^{\left(s_{2}, \ldots, t_{2}\right)} A^{\left(s_{1}, \ldots, t_{1}\right)}$, which will be used in Theorem 9.3 below.

Lemma 8.4 ( [87]). Let $A \in \mathbb{C}^{m \times n}$ and $B \in \mathbb{C}^{n \times p}$ be given, and denote $N=\left[A^{*}, B\right]$.

(a) The following 16 rank equalities always hold for all the generalized inverses

$$
\begin{aligned}
& r\left(B B^{\dagger} A^{\dagger} A\right)=r\left(B B^{\dagger} A^{(1,3,4)} A\right)=r\left(B B^{\dagger} A^{(1,2,4)} A\right)=r\left(B B^{\dagger} A^{(1,4)} A\right)=r\left(B B^{(1,3,4)} A^{\dagger} A\right) \\
& =r\left(B B^{(1,3,4)} A^{(1,3,4)} A\right)=r\left(B B^{(1,3,4)} A^{(1,2,4)} A\right)=r\left(B B^{(1,3,4)} A^{(1,4)} A\right)=r\left(B B^{(1,2,3)} A^{\dagger} A\right) \\
& =r\left(B B^{(1,2,3)} A^{(1,3,4)} A\right)=r\left(B B^{(1,2,3)} A^{(1,2,4)} A\right)=r\left(B B^{(1,2,3)} A^{(1,4)} A\right)=r\left(B B^{(1,3)} A^{\dagger} A\right) \\
& =r\left(B B^{(1,3)} A^{(1,3,4)} A\right)=r\left(B B^{(1,3)} A^{(1,2,4)} A\right)=r\left(B B^{(1,3)} A^{(1,4)} A\right)=r(A B) .
\end{aligned}
$$

(b) The following 48 maximum rank formulas hold

$$
\begin{aligned}
u & \left(B B^{\dagger} A^{(1,2,3)} A\right)=u\left(B B^{\dagger} A^{(1,3)} A\right)=u\left(B B^{\dagger} A^{(1,2)} A\right)=u\left(B B^{\dagger} A^{(1)} A\right) \\
= & u\left(B B^{(1,3,4)} A^{(1,2,3)} A\right)=u\left(B B^{(1,3,4)} A^{(1,3)} A\right)=u\left(B B^{(1,3,4)} A^{(1,2)} A\right)=u\left(B B^{(1,3,4)} A^{(1)} A\right) \\
= & u\left(B B^{(1,2,3)} A^{(1,2,3)} A\right)=u\left(B B^{(1,2,3)} A^{(1,3)} A\right)=u\left(B B^{(1,2,3)} A^{(1,2)} A\right)=u\left(B B^{(1,2,3)} A^{(1)} A\right) \\
= & u\left(B B^{(1,3)} A^{(1,2,3)} A\right)=u\left(B B^{(1,3)} A^{(1,3)} A\right)=u\left(B B^{(1,3)} A^{(1,2)} A\right)=u\left(B B^{(1,3)} A^{(1)} A\right) \\
= & u\left(B B^{(1,2,4)} A^{\dagger} A\right)=u\left(B B^{(1,2,4)} A^{(1,3,4)} A\right)=u\left(B B^{(1,2,4)} A^{(1,2,4)} A\right)=u\left(B B^{(1,2,4)} A^{(1,4)} A\right) \\
= & u\left(B B^{(1,4)} A^{\dagger} A\right)=u\left(B B^{(1,4)} A^{(1,3,4)} A\right)=u\left(B B^{(1,4)} A^{(1,2,4)} A\right)=u\left(B B^{(1,4)} A^{(1,4)} A\right) \\
= & u\left(B B^{(1,2)} A^{\dagger} A\right)=u\left(B B^{(1,2)} A^{(1,3,4)} A\right)=u\left(B B^{(1,2)} A^{(1,2,4)} A\right)=u\left(B B^{(1,2)} A^{(1,4)} A\right) \\
= & u\left(B B^{(1)} A^{\dagger} A\right)=u\left(B B^{(1)} A^{(1,3,4)} A\right)=u\left(B B^{(1)} A^{(1,2,4)} A\right)=u\left(B B^{(1)} A^{(1,4)} A\right) \\
= & u\left(B B^{(1,2,4)} A^{(1,2,3)} A\right)=u\left(B B^{(1,2,4)} A^{(1,3)} A\right)=u\left(B B^{(1,2,4)} A^{(1,2)} A\right)=u\left(B B^{(1,2,4)} A^{(1)} A\right) \\
= & u\left(B B^{(1,4)} A^{(1,2,3)} A\right)=u\left(B B^{(1,4)} A^{(1,3)} A\right)=u\left(B B^{(1,4)} A^{(1,2)} A\right)=u\left(B B^{(1,4)} A^{(1)} A\right) \\
& =u\left(B B^{(1,2)} A^{(1,2,3)} A\right)=u\left(B B^{(1,2)} A^{(1,3)} A\right)=u\left(B B^{(1,2)} A^{(1,2)} A\right)=u\left(B B^{(1,2)} A^{(1)} A\right) \\
& =u\left(B B^{(1)} A^{(1,2,3)} A\right)=u\left(B B^{(1)} A^{(1,3)} A\right)=u\left(B B^{(1)} A^{(1,2)} A\right)=u\left(B B^{(1)} A^{(1)} A\right) \\
& =\min \{r(A), \quad r(B)\} .
\end{aligned}
$$


(c) The following 32 minimum rank formulas hold

$$
\begin{aligned}
& v\left(B B^{\dagger} A^{(1,2,3)} A\right)=v\left(B B^{\dagger} A^{(1,3)} A\right)=v\left(B B^{\dagger} A^{(1,2)} A\right)=v\left(B B^{\dagger} A^{(1)} A\right) \\
& =v\left(B B^{(1,3,4)} A^{(1,2,3)} A\right)=v\left(B B^{(1,3,4)} A^{(1,3)} A\right)=v\left(B B^{(1,3,4)} A^{(1,2)} A\right)=v\left(B B^{(1,3,4)} A^{(1)} A\right) \\
& =v\left(B B^{(1,2,3)} A^{(1,2,3)} A\right)=v\left(B B^{(1,2,3)} A^{(1,3)} A\right)=v\left(B B^{(1,2,3)} A^{(1,2)} A\right)=v\left(B B^{(1,2,3)} A^{(1)} A\right) \\
& =v\left(B B^{(1,3)} A^{(1,2,3)} A\right)=v\left(B B^{(1,3)} A^{(1,3)} A\right)=v\left(B B^{(1,3)} A^{(1,2)} A\right)=v\left(B B^{(1,3)} A^{(1)} A\right) \\
& =v\left(B B^{(1,2,4)} A^{\dagger} A\right)=v\left(B B^{(1,2,4)} A^{(1,3,4)} A\right)=v\left(B B^{(1,2,4)} A^{(1,2,4)} A\right)=v\left(B B^{(1,2,4)} A^{(1,4)} A\right) \\
& =v\left(B B^{(1,4)} A^{\dagger} A\right)=v\left(B B^{(1,4)} A^{(1,3,4)} A\right)=v\left(B B^{(1,4)} A^{(1,2,4)} A\right)=v\left(B B^{(1,4)} A^{(1,4)} A\right) \\
& =v\left(B B^{(1,2)} A^{\dagger} A\right)=v\left(B B^{(1,2)} A^{(1,3,4)} A\right)=v\left(B B^{(1,2)} A^{(1,2,4)} A\right)=v\left(B B^{(1,2)} A^{(1,4)} A\right) \\
& =v\left(B B^{(1)} A^{\dagger} A\right)=v\left(B B^{(1)} A^{(1,3,4)} A\right)=v\left(B B^{(1)} A^{(1,2,4)} A\right)=v\left(B B^{(1)} A^{(1,4)} A\right) \\
& =r(A)+r(B)-r(N) ;
\end{aligned}
$$

the following 16 minimum rank formulas hold

$$
\begin{aligned}
& v\left(B B^{(1,2,4)} A^{(1,2,3)} A\right)=v\left(B B^{(1,2,4)} A^{(1,3)} A\right)=v\left(B B^{(1,2,4)} A^{(1,2)} A\right)=v\left(B B^{(1,2,4)} A^{(1)} A\right) \\
& =v\left(B B^{(1,4)} A^{(1,2,3)} A\right)=v\left(B B^{(1,4)} A^{(1,3)} A\right)=v\left(B B^{(1,4)} A^{(1,2)} A\right)=v\left(B B^{(1,4)} A^{(1)} A\right) \\
& =v\left(B B^{(1,2)} A^{(1,2,3)} A\right)=v\left(B B^{(1,2)} A^{(1,3)} A\right)=v\left(B B^{(1,2)} A^{(1,2)} A\right)=v\left(B B^{(1,2)} A^{(1)} A\right) \\
& =v\left(B B^{(1)} A^{(1,2,3)} A\right)=v\left(B B^{(1)} A^{(1,3)} A\right)=v\left(B B^{(1)} A^{(1,2)} A\right)=v\left(B B^{(1)} A^{(1)} A\right) \\
& =\max \{0, \quad r(A)+r(B)-n\} .
\end{aligned}
$$

(d) The following 16 formulas hold for all the generalized inverses

$$
\begin{aligned}
& r\left(I_{n}-B B^{\dagger} A^{\dagger} A\right)=r\left(I_{n}-B B^{\dagger} A^{(1,3,4)} A\right)=r\left(I_{n}-B B^{\dagger} A^{(1,2,4)} A\right) \\
& =r\left(I_{n}-B B^{\dagger} A^{(1,4)} A\right)=r\left(I_{n}-B B^{(1,3,4)} A^{\dagger} A\right)=r\left(I_{n}-B B^{(1,3,4)} A^{(1,3,4)} A\right) \\
& =r\left(I_{n}-B B^{(1,3,4)} A^{(1,2,4)} A\right)=r\left(I_{n}-B B^{(1,3,4)} A^{(1,4)} A\right)=r\left(I_{n}-B B^{(1,2,3)} A^{\dagger} A\right) \\
& =r\left(I_{n}-B B^{(1,2,3)} A^{(1,3,4)} A\right)=r\left(I_{n}-B B^{(1,2,3)} A^{(1,2,4)} A\right)=r\left(I_{n}-B B^{(1,2,3)} A^{(1,4)} A\right) \\
& =r\left(I_{n}-B B^{(1,3)} A^{\dagger} A\right)=r\left(I_{n}-B B^{(1,3)} A^{(1,3,4)} A\right)=r\left(I_{n}-B B^{(1,3)} A^{(1,2,4)} A\right) \\
& =r\left(I_{n}-B B^{(1,3)} A^{(1,4)} A\right)=r(N)-r(A)-r(B)+n .
\end{aligned}
$$

(e) The following 32 maximum rank formulas hold

$$
\begin{aligned}
& u\left(I_{n}-B B^{\dagger} A^{(1,2,3)} A\right)=u\left(I_{n}-B B^{\dagger} A^{(1,3)} A\right)=u\left(I_{n}-B B^{\dagger} A^{(1,2)} A\right) \\
& =u\left(I_{n}-B B^{\dagger} A^{(1)} A\right)=u\left(I_{n}-B B^{(1,3,4)} A^{(1,2,3)} A\right)=u\left(I_{n}-B B^{(1,3,4)} A^{(1,3)} A\right) \\
& =u\left(I_{n}-B B^{(1,3,4)} A^{(1,2)} A\right)=u\left(I_{n}-B B^{(1,3,4)} A^{(1)} A\right)=u\left(I_{n}-B B^{(1,2,3)} A^{(1,2,3)} A\right) \\
& =u\left(I_{n}-B B^{(1,2,3)} A^{(1,3)} A\right)=u\left(I_{n}-B B^{(1,2,3)} A^{(1,2)} A\right)=u\left(I_{n}-B B^{(1,2,3)} A^{(1)} A\right) \\
& =u\left(I_{n}-B B^{(1,3)} A^{(1,2,3)} A\right)=u\left(I_{n}-B B^{(1,3)} A^{(1,3)} A\right)=u\left(I_{n}-B B^{(1,3)} A^{(1,2)} A\right) \\
& =u\left(I_{n}-B B^{(1,3)} A^{(1)} A\right)=u\left(I_{n}-B B^{(1,2,4)} A^{\dagger} A\right)=u\left(I_{n}-B B^{(1,2,4)} A^{(1,3,4)} A\right) \\
& =u\left(I_{n}-B B^{(1,2,4)} A^{(1,2,4)} A\right)=u\left(I_{n}-B B^{(1,2,4)} A^{(1,4)} A\right)=u\left(I_{n}-B B^{(1,4)} A^{\dagger} A\right) \\
& =u\left(I_{n}-B B^{(1,4)} A^{(1,3,4)} A\right)=u\left(I_{n}-B B^{(1,4)} A^{(1,2,4)} A\right)=u\left(I_{n}-B B^{(1,4)} A^{(1,4)} A\right) \\
& =u\left(I_{n}-B B^{(1,2)} A^{\dagger} A\right)=u\left(I_{n}-B B^{(1,2)} A^{(1,3,4)} A\right)=u\left(I_{n}-B B^{(1,2)} A^{(1,2,4)} A\right) \\
& =u\left(I_{n}-B B^{(1,2)} A^{(1,4)} A\right)=u\left(I_{n}-B B^{(1)} A^{\dagger} A\right)=u\left(I_{n}-B B^{(1)} A^{(1,3,4)} A\right) \\
& =u\left(I_{n}-B B^{(1)} A^{(1,2,4)} A\right)=u\left(I_{n}-B B^{(1)} A^{(1,4)} A\right) \\
& =r(N)-r(A)-r(B)+n ;
\end{aligned}
$$


the following 16 maximum rank formulas hold

$$
\begin{aligned}
& u\left(I_{n}-B B^{(1,2,4)} A^{(1,2,3)} A\right)=u\left(I_{n}-B B^{(1,2,4)} A^{(1,3)} A\right)=u\left(I_{n}-B B^{(1,2,4)} A^{(1,2)} A\right) \\
& =u\left(I_{n}-B B^{(1,2,4)} A^{(1)} A\right)=u\left(I_{n}-B B^{(1,4)} A^{(1,2,3)} A\right)=u\left(I_{n}-B B^{(1,4)} A^{(1,3)} A\right) \\
& =u\left(I_{n}-B B^{(1,4)} A^{(1,2)} A\right)=u\left(I_{n}-B B^{(1,4)} A^{(1)} A\right)=u\left(I_{n}-B B^{(1,2)} A^{(1,2,3)} A\right) \\
& =u\left(I_{n}-B B^{(1,2)} A^{(1,3)} A\right)=u\left(I_{n}-B B^{(1,2)} A^{(1,2)} A\right)=u\left(I_{n}-B B^{(1,2)} A^{(1)} A\right) \\
& =u\left(I_{n}-B B^{(1)} A^{(1,2,3)} A\right)=u\left(I_{n}-B B^{(1)} A^{(1,3)} A\right)=u\left(I_{n}-B B^{(1)} A^{(1,2)} A\right) \\
& =u\left(I_{n}-B B^{(1)} A^{(1)} A\right)=\min \{n, \quad 2 n-r(A)-r(B)\} .
\end{aligned}
$$

(f) The following 48 minimum rank formulas hold

$$
\begin{aligned}
& v\left(I_{n}-B B^{\dagger} A^{(1,2,3)} A\right)=v\left(I_{n}-B B^{\dagger} A^{(1,3)} A\right)=v\left(I_{n}-B B^{\dagger} A^{(1,2)} A\right) \\
& =v\left(I_{n}-B B^{\dagger} A^{(1)} A\right)=v\left(I_{n}-B B^{(1,3,4)} A^{(1,2,3)} A\right)=v\left(I_{n}-B B^{(1,3,4)} A^{(1,3)} A\right) \\
& =v\left(I_{n}-B B^{(1,3,4)} A^{(1,2)} A\right)=v\left(I_{n}-B B^{(1,3,4)} A^{(1)} A\right)=v\left(I_{n}-B B^{(1,2,3)} A^{(1,2,3)} A\right) \\
& =v\left(I_{n}-B B^{(1,2,3)} A^{(1,3)} A\right)=v\left(I_{n}-B B^{(1,2,3)} A^{(1,2)} A\right)=v\left(I_{n}-B B^{(1,2,3)} A^{(1)} A\right) \\
& =v\left(I_{n}-B B^{(1,3)} A^{(1,2,3)} A\right)=v\left(I_{n}-B B^{(1,3)} A^{(1,3)} A\right)=v\left(I_{n}-B B^{(1,3)} A^{(1,2)} A\right) \\
& =v\left(I_{n}-B B^{(1,3)} A^{(1)} A\right)=v\left(I_{n}-B B^{(1,2,4)} A^{\dagger} A\right)=v\left(I_{n}-B B^{(1,2,4)} A^{(1,3,4)} A\right) \\
& =v\left(I_{n}-B B^{(1,2,4)} A^{(1,2,4)} A\right)=v\left(I_{n}-B B^{(1,2,4)} A^{(1,4)} A\right)=v\left(I_{n}-B B^{(1,4)} A^{\dagger} A\right) \\
& =v\left(I_{n}-B B^{(1,4)} A^{(1,3,4)} A\right)=v\left(I_{n}-B B^{(1,4)} A^{(1,2,4)} A\right)=v\left(I_{n}-B B^{(1,4)} A^{(1,4)} A\right) \\
& =v\left(I_{n}-B B^{(1,2)} A^{\dagger} A\right)=v\left(I_{n}-B B^{(1,2)} A^{(1,3,4)} A\right)=v\left(I_{n}-B B^{(1,2)} A^{(1,2,4)} A\right) \\
& =v\left(I_{n}-B B^{(1,2)} A^{(1,4)} A\right)=v\left(I_{n}-B B^{(1)} A^{\dagger} A\right)=v\left(I_{n}-B B^{(1)} A^{(1,3,4)} A\right) \\
& =v\left(I_{n}-B B^{(1)} A^{(1,2,4)} A\right)=v\left(I_{n}-B B^{(1)} A^{(1,4)} A\right)=v\left(I_{n}-B B^{(1,2,4)} A^{(1,2,3)} A\right) \\
& =v\left(I_{n}-B B^{(1,2,4)} A^{(1,3)} A\right)=v\left(I_{n}-B B^{(1,2,4)} A^{(1,2)} A\right)=v\left(I_{n}-B B^{(1,2,4)} A^{(1)} A\right) \\
& =v\left(I_{n}-B B^{(1,4)} A^{(1,2,3)} A\right)=v\left(I_{n}-B B^{(1,4)} A^{(1,3)} A\right)=v\left(I_{n}-B B^{(1,4)} A^{(1,2)} A\right) \\
& =v\left(I_{n}-B B^{(1,4)} A^{(1)} A\right)=v\left(I_{n}-B B^{(1,2)} A^{(1,2,3)} A\right)=v\left(I_{n}-B B^{(1,2)} A^{(1,3)} A\right) \\
& =v\left(I_{n}-B B^{(1,2)} A^{(1,2)} A\right)=v\left(I_{n}-B B^{(1,2)} A^{(1)} A\right)=v\left(I_{n}-B B^{(1)} A^{(1,2,3)} A\right) \\
& =v\left(I_{n}-B B^{(1)} A^{(1,3)} A\right)=v\left(I_{n}-B B^{(1)} A^{(1,2)} A\right)=v\left(I_{n}-B B^{(1)} A^{(1)} A\right) \\
& =n-r(A B) .
\end{aligned}
$$

(g) The ranks of the 64 products $A^{(i, \ldots, j)} A B B^{\left(s_{2}, \ldots, t_{2}\right)}$ are fixed for all the eight commonly-used types of $A^{(i, \ldots, j)}$ and $B^{\left(s_{2}, \ldots, t_{2}\right)}$, and satisfy

$$
r\left(A^{(i, \ldots, j)} A B B^{\left(s_{2}, \ldots, t_{2}\right)}\right)=r(A B)
$$

(h) The following 16 rank equalities hold for all the generalized inverses

$$
\begin{aligned}
& r\left(I_{n}-A^{\dagger} A B B^{\dagger}\right)=r\left(I_{n}-A^{(1,3,4)} A B B^{\dagger}\right)=r\left(I_{n}-A^{(1,2,4)} A B B^{\dagger}\right) \\
& =r\left(I_{n}-A^{(1,4)} A B B^{\dagger}\right)=r\left(I_{n}-B B^{(1,3,4)} A^{\dagger} A\right)=r\left(I_{n}-A^{(1,3,4)} A B B^{(1,3,4)}\right) \\
& =r\left(I_{n}-A^{(1,2,4)} A B B^{(1,3,4)}\right)=r\left(I_{n}-A^{(1,4)} A B B^{(1,3,4)}=r\left(I_{n}-A^{\dagger} A B B^{(1,2,3)}\right)\right. \\
& =r\left(I_{n}-A^{(1,3,4)} A B B^{(1,2,3)}\right)=r\left(I_{n}-A^{(1,2,4)} A B B^{(1,2,3)}\right)=r\left(I_{n}-A^{(1,4)} A B B^{(1,2,3)}\right) \\
& =r\left(I_{n}-A^{\dagger} A B B^{(1,3)}\right)=r\left(I_{n}-A^{(1,3)} A B B^{(1,3)}\right)=r\left(I_{n}-A^{(1,2,4)} A B B^{(1,3)}\right) \\
& =r\left(I_{n}-A^{(1,4)} A B B^{(1,3)}\right)=r(N)-r(A)-r(B)+n .
\end{aligned}
$$

(i) The following 32 maximum rank formulas hold

$$
\begin{aligned}
& u\left(I_{n}-A^{(1,2,3)} A B B^{\dagger}\right)=u\left(I_{n}-A^{(1,3)} A B B^{\dagger}\right)=u\left(I_{n}-A^{(1,2)} A B B^{\dagger}\right) \\
& =u\left(I_{n}-A^{(1)} A B B^{\dagger}\right)=u\left(I_{n}-A^{(1,2,3)} A B B^{(1,3,4)}\right)=u\left(I_{n}-A^{(1,3)} A B B^{(1,3,4)}\right)
\end{aligned}
$$




$$
\begin{aligned}
& =u\left(I_{n}-A^{(1,2)} A B B^{(1,3,4)}\right)=u\left(I_{n}-A^{(1)} A B B^{(1,3,4)}\right)=u\left(I_{n}-A^{(1,2,3)} A B B^{(1,2,3)}\right) \\
& =u\left(I_{n}-A^{(1,3)} A B B^{(1,2,3)}\right)=u\left(I_{n}-A^{(1,2)} A B B^{(1,2,3)}\right)=u\left(I_{n}-A^{(1)} A B B^{(1,2,3)}\right) \\
& =u\left(I_{n}-A^{(1,2,3)} A B B^{(1,3)}\right)=u\left(I_{n}-A^{(1,3)} A B B^{(1,3)}\right)=u\left(I_{n}-A^{(1,2)} A B B^{(1,3)}\right) \\
& =u\left(I_{n}-A^{(1)} A B B^{(1,3)}\right)=u\left(I_{n}-A^{\dagger} A B B^{(1,2,4)}\right)=u\left(I_{n}-A^{(1,3,4)} A B B^{(1,2,4)}\right) \\
& =u\left(I_{n}-A^{(1,2,4)} A B B^{(1,2,4)}\right)=u\left(I_{n}-A^{(1,4)} A B B^{(1,2,4)}\right)=u\left(I_{n}-A^{\dagger} A B B^{(1,4)}\right) \\
& =u\left(I_{n}-A^{(1,3,4)} A B B^{(1,4)}\right)=u\left(I_{n}-A^{(1,2,4)} A B B^{(1,4)}\right)=u\left(I_{n}-A^{(1,4)} A B B^{(1,4)}\right) \\
& =u\left(I_{n}-A^{\dagger} A B B^{(1,2)}\right)=u\left(I_{n}-A^{(1,3,4)} A B B^{(1,2)}\right)=u\left(I_{n}-A^{(1,2,4)} A B B^{(1,2)}\right) \\
& =u\left(I_{n}-A^{(1,4)} A B B^{(1,2)}\right)=u\left(I_{n}-A^{\dagger} A B B^{(1)}\right)=u\left(I_{n}-A^{(1,3,4)} A B B^{(1)}\right) \\
& =u\left(I_{n}-A^{(1,2,4)} A B B^{(1)}\right)=u\left(I_{n}-A^{(1,4)} A B B^{(1)}\right)=r(N)-r(A)-r(B)+n
\end{aligned}
$$

the following 16 maximum rank formulas hold

$$
\begin{aligned}
& u\left(I_{n}-A^{(1,2,3)} A B B^{(1,2,4)}\right)=u\left(I_{n}-A^{(1,3)} A B B^{(1,2,4)}\right)=u\left(I_{n}-A^{(1,2)} A B B^{(1,2,4)}\right) \\
& =u\left(I_{n}-A^{(1)} A B B^{(1,2,4)}\right)=u\left(I_{n}-A^{(1,2,3)} A B B^{(1,4)}\right)=u\left(I_{n}-A^{(1,3)} A B B^{(1,4)}\right) \\
& =u\left(I_{n}-A^{(1,2)} A B B^{(1,4)}\right)=u\left(I_{n}-A^{(1)} A B B^{(1,4)}\right)=u\left(I_{n}-A^{(1,2,3)} A B B^{(1,2)}\right) \\
& =u\left(I_{n}-A^{(1,3)} A B B^{(1,2)}\right)=u\left(I_{n}-A^{(1,2)} A B B^{(1,2)}\right)=u\left(I_{n}-A^{(1)} A B B^{(1,2)}\right) \\
& =u\left(I_{n}-A^{(1,2,3)} A B B^{(1)}\right)=u\left(I_{n}-A^{(1,3)} A B B^{(1)}\right)=u\left(I_{n}-A^{(1,2)} A B B^{(1)}\right) \\
& =u\left(I_{n}-A^{(1)} A B B^{(1)}\right)=\min \{n, \quad 2 n-r(A)-r(B)\} .
\end{aligned}
$$

(j) The following 48 minimum rank formulas hold

$$
\begin{aligned}
& v\left(I_{n}-A^{(1,2,3)} A B B^{\dagger}\right)=v\left(I_{n}-A^{(1,3)} A B B^{\dagger}\right)=v\left(I_{n}-A^{(1,2)} A B B^{\dagger}\right) \\
& =v\left(I_{n}-A^{(1)} A B B^{\dagger}\right)=v\left(I_{n}-A^{(1,2,3)} A B B^{(1,3,4)}\right)=v\left(I_{n}-A^{(1,3)} A B B^{(1,3,4)}\right) \\
& =v\left(I_{n}-A^{(1,2)} A B B^{(1,3,4)}\right)=v\left(I_{n}-A^{(1)} A B B^{(1,3,4)}\right)=v\left(I_{n}-A^{(1,2,3)} A B B^{(1,2,3)}\right) \\
& =v\left(I_{n}-A^{(1,3)} A B B^{(1,2,3)}\right)=v\left(I_{n}-A^{(1,2)} A B B^{(1,2,3)}\right)=v\left(I_{n}-A^{(1)} A B B^{(1,2,3)}\right) \\
& =v\left(I_{n}-A^{(1,2,3)} A B B^{(1,3)}\right)=v\left(I_{n}-A^{(1,3)} A B B^{(1,3)}\right)=v\left(I_{n}-A^{(1,2)} A B B^{(1,3)}\right) \\
& =v\left(I_{n}-A^{(1)} A B B^{(1,3)}\right)=v\left(I_{n}-A^{\dagger} A B B^{(1,2,4)}\right)=v\left(I_{n}-A^{(1,3,4)} A B B^{(1,2,4)}\right) \\
& =v\left(I_{n}-A^{(1,2,4)} A B B^{(1,2,4)}\right)=v\left(I_{n}-A^{(1,4)} A B B^{(1,2,4)}\right)=v\left(I_{n}-A^{\dagger} A B B^{(1,4)}\right) \\
& =v\left(I_{n}-A^{(1,3,4)} A B B^{(1,4)}\right)=v\left(I_{n}-A^{(1,2,4)} A B B^{(1,4)}\right)=v\left(I_{n}-A^{(1,4)} A B B^{(1,4)}\right) \\
& =v\left(I_{n}-A^{\dagger} A B B^{(1,2)}\right)=v\left(I_{n}-A^{(1,3,4)} A B B^{(1,2)}\right)=v\left(I_{n}-A^{(1,2,4)} A B B^{(1,2)}\right)= \\
& =v\left(I_{n}-A^{(1,4)} A B B^{(1,2)}\right)=v\left(I_{n}-A^{\dagger} A B B^{(1)}\right)=v\left(I_{n}-A^{(1,3,4)} A B B^{(1)}\right) \\
& =v\left(I_{n}-A^{(1,2,4)} A B B^{(1)}\right)=v\left(I_{n}-A^{(1,4)} A B B^{(1)}\right)=v\left(I_{n}-A^{(1,2,3)} A B B^{(1,2,4)}\right) \\
& =v\left(I_{n}-A^{(1,3)} A B B^{(1,2,4)}\right)=v\left(I_{n}-A^{(1,2)} A B B^{(1,2,4)}\right)=v\left(I_{n}-A^{(1)} A B B^{(1,2,4)}\right) \\
& =v\left(I_{n}-A^{(1,2,3)} A B B^{(1,4)}\right)=v\left(I_{n}-A^{(1,3)} A B B^{(1,4)}\right)=v\left(I_{n}-A^{(1,2)} A B B^{(1,4)}\right) \\
& =v\left(I_{n}-A^{(1)} A B B^{(1,4)}\right)=v\left(I_{n}-A^{(1,2,3)} A B B^{(1,2)}\right)=v\left(I_{n}-A^{(1,3)} A B B^{(1,2)}\right) \\
& =v\left(I_{n}-A^{(1,2)} A B B^{(1,2)}\right)=v\left(I_{n}-A^{(1)} A B B^{(1,2)}\right)=v\left(I_{n}-A^{(1,2,3)} A B B^{(1)}\right) \\
& =v\left(I_{n}-A^{(1,3)} A B B^{(1)}\right)=v\left(I_{n}-A^{(1,2)} A B B^{(1)}\right)=v\left(I_{n}-A^{(1)} A B B^{(1)}\right)=n-r(A B) .
\end{aligned}
$$

(k) The following 64 rank equalities hold for the eight commonly-used types of generalized inverses $A^{(i, . ., j)}$ and $B^{\left(s_{2}, \ldots, t_{2}\right)}$

$$
u\left(A B B^{\left(s_{2}, \ldots, t_{2}\right)} A^{(i, \ldots, j)}\right)=r(A B) .
$$

(1) The following 16 minimum rank formulas hold

$$
\begin{aligned}
& v\left(A B B^{(1,3,4)} A^{\dagger}\right)=v\left(A B B^{(1,2,3)} A^{\dagger}\right)=v\left(A B B^{(1,3)} A^{\dagger}\right)=v\left(A B B^{\dagger} A^{(1,3,4)}\right) \\
& =v\left(A B B^{(1,3,4)} A^{(1,3,4)}\right)=v\left(A B B^{(1,2,3)} A^{(1,3,4)}\right)=v\left(A B B^{(1,3)} A^{(1,3,4)}\right)=v\left(A B B^{\dagger} A^{(1,2,4)}\right) \\
& =v\left(A B B^{(1,3,4)} A^{(1,2,4)}\right)=v\left(A B B^{(1,2,3)} A^{(1,2,4)}\right)=v\left(A B B^{(1,3)} A^{(1,2,4)}\right)=v\left(A B B^{\dagger} A^{(1,4)}\right) \\
& =v\left(A B B^{(1,3,4)} A^{(1,4)}\right)=v\left(A B B^{(1,2,3)} A^{(1,4)}\right)=v\left(A B B^{(1,3)} A^{(1,4)}\right)=r(A B) ;
\end{aligned}
$$


the following 32 minimum rank formulas hold

$$
\begin{aligned}
& v\left(A B B^{(1,2,4)} A^{\dagger}\right)=v\left(A B B^{(1,4)} A^{\dagger}\right)=v\left(A B B^{(1,2)} A^{\dagger}\right)=v\left(A B B^{(1)} A^{\dagger}\right) \\
& =v\left(A B B^{(1,2,4)} A^{(1,3,4)}\right)=v\left(A B B^{(1,4)} A^{(1,3,4)}\right)=v\left(A B B^{(1,2)} A^{(1,3,4)}\right)=v\left(A B B^{(1)} A^{(1,3,4)}\right) \\
& =v\left(A B B^{(1,2,4)} A^{(1,2,4)}\right)=v\left(A B B^{(1,4)} A^{(1,2,4)}\right)=v\left(A B B^{(1,2)} A^{(1,2,4)}\right)=v\left(A B B^{(1)} A^{(1,2,4)}\right) \\
& =v\left(A B B^{(1,2,4)} A^{(1,4)}\right)=v\left(A B B^{(1,4)} A^{(1,4)}\right)=v\left(A B B^{(1,2)} A^{(1,4)}\right)=v\left(A B B^{(1)} A^{(1,4)}\right) \\
& =v\left(A B B^{\dagger} A^{(1,2,3)}\right)=v\left(A B B^{(1,3,4)} A^{(1,2,3)}\right)=v\left(A B B^{(1,2,3)} A^{(1,2,3)}\right)=v\left(A B B^{(1,3)} A^{(1,2,3)}\right) \\
& =v\left(A B B^{\dagger} A^{(1,3)}\right)=v\left(A B B^{(1,3,4)} A^{(1,3)}\right)=v\left(A B B^{(1,2,3)} A^{(1,3)}\right)=v\left(A B B^{(1,3)} A^{(1,3)}\right) \\
& =v\left(A B B^{\dagger} A^{(1,2)}\right)=v\left(A B B^{(1,3,4)} A^{(1,2)}\right)=v\left(A B B^{(1,2,3)} A^{(1,2)}\right)=v\left(A B B^{(1,3)} A^{(1,2)}\right) \\
& =v\left(A B B^{\dagger} A^{(1)}\right)=v\left(A B B^{(1,3,4)} A^{(1)}\right)=v\left(A B B^{(1,2,3)} A^{(1)}\right)=v\left(A B B^{(1,3)} A^{(1)}\right) \\
& =r(A)+r(B)-r(N)
\end{aligned}
$$

the following 16 maximum rank formulas hold

$$
\begin{aligned}
& v\left(A B B^{(1,2,4)} A^{(1,2,3)}\right)=v\left(A B B^{(1,4)} A^{(1,2,3)}\right)=v\left(A B B^{(1,2)} A^{(1,2,3)}\right)=v\left(A B B^{(1)} A^{(1,2,3)}\right) \\
& =v\left(A B B^{(1,2,4)} A^{(1,3)}\right)=v\left(A B B^{(1,4)} A^{(1,3)}\right)=v\left(A B B^{(1,2)} A^{(1,3)}\right)=v\left(A B B^{(1)} A^{(1,3)}\right) \\
& =v\left(A B B^{(1,2,4)} A^{(1,2)}\right)=v\left(A B B^{(1,4)} A^{(1,2)}\right)=v\left(A B B^{(1,2)} A^{(1,2)}\right)=v\left(A B B^{(1)} A^{(1,2)}\right) \\
& =v\left(A B B^{(1,2,4)} A^{(1)}\right)=v\left(A B B^{(1,4)} A^{(1)}\right)=v\left(A B B^{(1,2)} A^{(1)}\right)=v\left(A B B^{(1)} A^{(1)}\right) \\
& =\max \{0, \quad r(A)+r(B)-n\} .
\end{aligned}
$$

(m) The following 16 rank equalities hold for all the generalized inverses

$$
\begin{aligned}
& r\left(I_{m}-A B B^{\dagger} A^{\dagger}\right)=r\left(I_{m}-A B B^{(1,3,4)} A^{\dagger}\right)=r\left(I_{m}-A B B^{(1,2,3)} A^{\dagger}\right) \\
& =r\left(I_{m}-A B B^{(1,3)} A^{\dagger}\right)=r\left(I_{m}-A B B^{\dagger} A^{(1,3,4)}\right)=r\left(I_{m}-A B B^{(1,3,4)} A^{(1,3,4)}\right) \\
& =r\left(I_{m}-A B B^{(1,2,3)} A^{(1,3,4)}\right)=r\left(I_{m}-A B B^{(1,3)} A^{(1,3,4)}\right)=r\left(I_{m}-A B B^{\dagger} A^{(1,2,4)}\right) \\
& =r\left(I_{m}-A B B^{(1,3,4)} A^{(1,2,4)}\right)=r\left(I_{m}-A B B^{(1,2,3)} A^{(1,2,4)}\right)=r\left(I_{m}-A B B^{(1,3)} A^{(1,2,4)}\right) \\
& =r\left(I_{m}-A B B^{\dagger} A^{(1,4)}\right)=r\left(I_{m}-A B B^{(1,3,4)} A^{(1,4)}\right)=r\left(I_{m}-A B B^{(1,2,3)} A^{(1,4)}\right) \\
& =r\left(I_{m}-A B B^{(1,3)} A^{(1,4)}\right)=r(N)-r(A)-r(B)+m .
\end{aligned}
$$

(n) The following 32 maximum rank formulas hold

$$
\begin{aligned}
& u\left(I_{m}-A B B^{\dagger} A^{(1,2,3)}\right)=u\left(I_{m}-A B B^{(1,3,4)} A^{(1,2,3)}\right)=u\left(I_{m}-A B B^{(1,2,3)} A^{(1,2,3)}\right) \\
& =u\left(I_{m}-A B B^{(1,3)} A^{(1,2,3)}\right)=u\left(I_{m}-A B B^{\dagger} A^{(1,3)}\right)=u\left(I_{m}-A B B^{(1,3,4)} A^{(1,3)}\right) \\
& =u\left(I_{m}-A B B^{(1,2,3)} A^{(1,3)}\right)=u\left(I_{m}-A B B^{(1,3)} A^{(1,3)}\right)=u\left(I_{m}-A B B^{\dagger} A^{(1,2)}\right) \\
& =u\left(I_{m}-A B B^{(1,3,4)} A^{(1,2)}\right)=u\left(I_{m}-A B B^{(1,2,3)} A^{(1,2)}\right)=u\left(I_{m}-A B B^{(1,3)} A^{(1,2)}\right) \\
& =u\left(I_{m}-A B B^{\dagger} A^{(1)}\right)=u\left(I_{m}-A B B^{(1,3,4)} A^{(1)}\right)=u\left(I_{m}-A B B^{(1,2,3)} A^{(1)}\right) \\
& =u\left(I_{m}-A B B^{(1,3)} A^{(1)}\right)=u\left(I_{m}-A B B^{(1,2,4)} A^{\dagger}\right)=u\left(I_{m}-A B B^{(1,4)} A^{\dagger}\right) \\
& =u\left(I_{m}-A B B^{(1,2)} A^{\dagger}\right)=u\left(I_{m}-A B B^{(1)} A^{\dagger}\right)=u\left(I_{m}-A B B^{(1,2,4)} A^{(1,3,4)}\right) \\
& =u\left(I_{m}-A B B^{(1,4)} A^{(1,3,4)}\right)=u\left(I_{m}-A B B^{(1,2)} A^{(1,3,4)}\right)=u\left(I_{m}-A B B^{(1)} A^{(1,3,4)}\right) \\
& =u\left(I_{m}-A B B^{(1,2,4)} A^{(1,2,4)}\right)=u\left(I_{m}-A B B^{(1,4)} A^{(1,2,4)}\right)=u\left(I_{m}-A B B^{(1,2)} A^{(1,2,4)}\right) \\
& =u\left(I_{m}-A B B^{(1)} A^{(1,2,4)}\right)=u\left(I_{m}-A B B^{(1,2,4)} A^{(1,4)}\right)=u\left(I_{m}-A B B^{(1,4)} A^{(1,4)}\right) \\
& =u\left(I_{m}-A B B^{(1,2)} A^{(1,4)}\right)=u\left(I_{m}-A B B^{(1)} A^{(1,4)}\right)=r(N)-r(A)-r(B)+m ;
\end{aligned}
$$

the following 16 maximum rank formulas hold

$$
\begin{aligned}
& u\left(I_{m}-A B B^{(1,2,4)} A^{(1,2,3)}\right)=u\left(I_{m}-A B B^{(1,4)} A^{(1,2,3)}\right)=u\left(I_{m}-A B B^{(1,2)} A^{(1,2,3)}\right) \\
& =u\left(I_{m}-A B B^{(1)} A^{(1,2,3)}\right)=u\left(I_{m}-A B B^{(1,2,4)} A^{(1,3)}\right)=u\left(I_{m}-A B B^{(1,4)} A^{(1,3)}\right) \\
& =u\left(I_{m}-A B B^{(1,2)} A^{(1,3)}\right)=u\left(I_{m}-A B B^{(1)} A^{(1,3)}\right)=u\left(I_{m}-A B B^{(1,2,4)} A^{(1,2)}\right) \\
& =u\left(I_{m}-A B B^{(1,4)} A^{(1,2)}\right)=u\left(I_{m}-A B B^{(1,2)} A^{(1,2)}\right)=u\left(I_{m}-A B B^{(1)} A^{(1,2)}\right)
\end{aligned}
$$




$$
\begin{aligned}
& =u\left(I_{m}-A B B^{(1,2,4)} A^{(1)}\right)=u\left(I_{m}-A B B^{(1,4)} A^{(1)}\right)=u\left(I_{m}-A B B^{(1,2)} A^{(1)}\right) \\
& =u\left(I_{m}-A B B^{(1)} A^{(1)}\right)=\min \{m, \quad m+n-r(A)-r(B)\} .
\end{aligned}
$$

(o) The following 48 minimum rank formulas hold

$$
\begin{aligned}
& v\left(I_{m}-A B B^{\dagger} A^{(1,2,3)}\right)=v\left(I_{m}-A B B^{(1,3,4)} A^{(1,2,3)}\right)=v\left(I_{m}-A B B^{(1,2,3)} A^{(1,2,3)}\right) \\
& =v\left(I_{m}-A B B^{(1,3)} A^{(1,2,3)}\right)=v\left(I_{m}-A B B^{\dagger} A^{(1,3)}\right)=v\left(I_{m}-A B B^{(1,3,4)} A^{(1,3)}\right) \\
& =v\left(I_{m}-A B B^{(1,2,3)} A^{(1,3)}\right)=v\left(I_{m}-A B B^{(1,3)} A^{(1,3)}\right)=v\left(I_{m}-A B B^{\dagger} A^{(1,2)}\right) \\
& =v\left(I_{m}-A B B^{(1,3,4)} A^{(1,2)}\right)=v\left(I_{m}-A B B^{(1,2,3)} A^{(1,2)}\right)=v\left(I_{m}-A B B^{(1,3)} A^{(1,2)}\right) \\
& =v\left(I_{m}-A B B^{\dagger} A^{(1)}\right)=v\left(I_{m}-A B B^{(1,3,4)} A^{(1)}\right)=v\left(I_{m}-A B B^{(1,2,3)} A^{(1)}\right) \\
& =v\left(I_{m}-A B B^{(1,3)} A^{(1)}\right)=v\left(I_{m}-A B B^{(1,2,4)} A^{\dagger}\right)=v\left(I_{m}-A B B^{(1,4)} A^{\dagger}\right) \\
& =v\left(I_{m}-A B B^{(1,2)} A^{\dagger}\right)=v\left(I_{m}-A B B^{(1)} A^{\dagger}\right)=v\left(I_{m}-A B B^{(1,2,4)} A^{(1,3,4)}\right) \\
& =v\left(I_{m}-A B B^{(1,4)} A^{(1,3,4)}\right)=v\left(I_{m}-A B B^{(1,2)} A^{(1,3,4)}\right)=v\left(I_{m}-A B B^{(1)} A^{(1,3,4)}\right) \\
& =v\left(I_{m}-A B B^{(1,2,4)} A^{(1,2,4)}\right)=b e t a\left(I_{m}-A B B^{(1,4)} A^{(1,2,4)}\right)=v\left(I_{m}-A B B^{(1,2)} A^{(1,2,4)}\right) \\
& =v\left(I_{m}-A B B^{(1)} A^{(1,2,4)}\right)=v\left(I_{m}-A B B^{(1,2,4)} A^{(1,4)}\right)=v\left(I_{m}-A B B^{(1,4)} A^{(1,4)}\right) \\
& =v\left(I_{m}-A B B^{(1,2)} A^{(1,4)}\right)=v\left(I_{m}-A B B^{(1)} A^{(1,4)}\right)=v\left(I_{m}-A B B^{(1,2,4)} A^{(1,2,3)}\right) \\
& =v\left(I_{m}-A B B^{(1,4)} A^{(1,2,3)}\right)=v\left(I_{m}-A B B^{(1,2)} A^{(1,2,3)}\right)=v\left(I_{m}-A B B^{(1)} A^{(1,2,3)}\right) \\
& =v\left(I_{m}-A B B^{(1,2,4)} A^{(1,3)}\right)=v\left(I_{m}-A B B^{(1,4)} A^{(1,3)}\right)=v\left(I_{m}-A B B^{(1,2)} A^{(1,3)}\right) \\
& =v\left(I_{m}-A B B^{(1)} A^{(1,3)}\right)=v\left(I_{m}-A B B^{(1,2,4)} A^{(1,2)}\right)=v\left(I_{m}-A B B^{(1,4)} A^{(1,2)}\right) \\
& =v\left(I_{m}-A B B^{(1,2)} A^{(1,2)}\right)=v\left(I_{m}-A B B^{(1)} A^{(1,2)}\right)=v\left(I_{m}-A B B^{(1,2,4)} A^{(1)}\right) \\
& =v\left(I_{m}-A B B^{(1,4)} A^{(1)}\right)=v\left(I_{m}-A B B^{(1,2)} A^{(1)}\right)=v\left(I_{m}-A B B^{(1)} A^{(1)}\right) \\
& =m-r(A B) .
\end{aligned}
$$

(p) The following 64 rank equalities hold for the eight commonly-used types of generalized inverses $A^{(i, \ldots, j)}$ and $B^{\left(s_{2}, \ldots, t_{2}\right)}$

$$
u\left(B^{\left(s_{2}, \ldots, t_{2}\right)} A^{(i, \ldots, j)} A B\right)=r(A B) .
$$

(q) The following 16 minimum rank formulas hold

$$
\begin{aligned}
& v\left(B^{\dagger} A^{(1,3,4)} A B\right)=v\left(B^{\dagger} A^{(1,2,4)} A B\right)=v\left(B^{\dagger} A^{(1,4)} A B\right)=v\left(B^{(1,3,4)} A^{\dagger} A B\right) \\
& =v\left(B^{(1,3,4)} A^{(1,3,4)} A B\right)=v\left(B^{(1,3,4)} A^{(1,2,4)} A B\right)=v\left(B^{(1,3,4)} A^{(1,4)} A B\right)=v\left(B^{(1,2,3)} A^{\dagger} A B\right) \\
& =v\left(B^{(1,2,3)} A^{(1,3,4)} A B\right)=v\left(B^{(1,2,3)} A^{(1,2,4)} A B\right)=v\left(B^{(1,2,3)} A^{(1,4)} A B\right)=v\left(B^{(1,3)} A^{\dagger} A B\right) \\
& =v\left(B^{(1,3)} A^{(1,3,4)} A B\right)=v\left(B^{(1,3)} A^{(1,2,4)} A B\right)=v\left(B^{(1,3)} A^{(1,4)} A B\right)=r(A B) ;
\end{aligned}
$$

the following 32 maximum rank formulas hold

$$
\begin{aligned}
& v\left(B^{\dagger} A^{(1,2,3)} A B\right)=v\left(B^{\dagger} A^{(1,3)} A B\right)=v\left(B^{\dagger} A^{(1,2)} A B\right)=v\left(B^{\dagger} A^{(1)} A B\right) \\
& =v\left(B^{(1,3,4)} A^{(1,2,3)} A B\right)=v\left(B^{(1,3,4)} A^{(1,3)} A B\right)=v\left(B^{(1,3,4)} A^{(1,2)} A B\right)=v\left(B^{(1,3,4)} A^{(1)} A B\right) \\
& =v\left(B^{(1,2,3)} A^{(1,2,3)} A B\right)=v\left(B^{(1,2,3)} A^{(1,3)} A B\right)=v\left(B^{(1,2,3)} A^{(1,2)} A B\right)=v\left(B^{(1,2,3)} A^{(1)} A B\right) \\
& =v\left(B^{(1,3)} A^{(1,2,3)} A B\right)=v\left(B^{(1,3)} A^{(1,3)} A B\right)=v\left(B^{(1,3)} A^{(1,2)} A B\right)=v\left(B^{(1,3)} A^{(1)} A B\right) \\
& =v\left(B^{(1,2,4)} A^{\dagger} A B\right)=v\left(B^{(1,2,4)} A^{(1,3,4)} A B\right)=v\left(B^{(1,2,4)} A^{(1,2,4)} A B\right)=v\left(B^{(1,2,4)} A^{(1,4)} A B\right) \\
& =v\left(B^{(1,4)} A^{\dagger} A B\right)=v\left(B^{(1,4)} A^{(1,3,4)} A B\right)=v\left(B^{(1,4)} A^{(1,2,4)} A B\right)=v\left(B^{(1,4)} A^{(1,4)} A B\right) \\
& =v\left(B^{(1,2)} A^{\dagger} A B\right)=v\left(B^{(1,2)} A^{(1,3,4)} A B\right)=v\left(B^{(1,2)} A^{(1,2,4)} A B\right)=v\left(B^{(1,2)} A^{(1,4)} A B\right) \\
& =v\left(B^{(1)} A^{\dagger} A B\right)=v\left(B^{(1)} A^{(1,3,4)} A B\right)=v\left(B^{(1)} A^{(1,2,4)} A B\right)=v\left(B^{(1)} A^{(1,4)} A B\right) \\
& =r(A)+r(B)-r(N) ;
\end{aligned}
$$


the following 16 maximum rank formulas hold

$$
\begin{aligned}
& v\left(B^{(1,2,4)} A^{(1,2,3)} A B\right)=v\left(B^{(1,2,4)} A^{(1,3)} A B\right)=v\left(B^{(1,2,4)} A^{(1,2)} A B\right)=v\left(B^{(1,2,4)} A^{(1)} A B\right) \\
& =v\left(B^{(1,4)} A^{(1,2,3)} A B\right)=v\left(B^{(1,4)} A^{(1,3)} A B\right)=v\left(B^{(1,4)} A^{(1,2)} A B\right)=v\left(B^{(1,4)} A^{(1)} A B\right) \\
& =v\left(B^{(1,2)} A^{(1,2,3)} A B\right)=v\left(B^{(1,2)} A^{(1,3)} A B\right)=v\left(B^{(1,2)} A^{(1,2)} A B\right)=v\left(B^{(1,2)} A^{(1)} A B\right) \\
& =v\left(B^{(1)} A^{(1,2,3)} A B\right)=v\left(B^{(1)} A^{(1,3)} A B\right)=v\left(B^{(1)} A^{(1,2)} A B\right)=v\left(B^{(1)} A^{(1)} A B\right) \\
& =\max \{0, \quad r(A)+r(B)-n\} .
\end{aligned}
$$

(r) The following 16 rank equalities hold for all the generalized inverses

$$
\begin{aligned}
& r\left(I_{p}-B^{\dagger} A^{\dagger} A B\right)=r\left(I_{p}-B^{\dagger} A^{(1,3,4)} A B\right)=r\left(I_{p}-B^{\dagger} A^{(1,2,4)} A B\right) \\
& =r\left(I_{p}-B^{\dagger} A^{(1,4)} A B\right)=r\left(I_{p}-B^{(1,3,4)} A^{\dagger} A B\right)=r\left(I_{p}-B^{(1,3,4)} A^{(1,3,4)} A B\right) \\
& =r\left(I_{p}-B^{(1,3,4)} A^{(1,2,4)} A B\right)=r\left(I_{p}-B^{(1,3,4)} A^{(1,4)} A B\right)=r\left(I_{p}-B^{(1,2,3)} A^{\dagger} A B\right) \\
& =r\left(I_{p}-B^{(1,2,3)} A^{(1,3,4)} A B\right)=r\left(I_{p}-B^{(1,2,3)} A^{(1,2,4)} A B\right)=r\left(I_{p}-B^{(1,2,3)} A^{(1,4)} A B\right) \\
& =r\left(I_{p}-B^{(1,3)} A^{(\dagger)} A B\right)=r\left(I_{p}-B^{(1,3)} A^{(1,3,4)} A B\right)=r\left(I_{p}-B^{(1,3)} A^{(1,2,4)} A B\right) \\
& =r\left(I_{p}-B^{(1,3)} A^{(1,4)} A B\right)=r(N)-r(A)-r(B)+p .
\end{aligned}
$$

(s) The following 32 maximum rank formulas hold

$$
\begin{aligned}
& u\left(I_{p}-B^{(1,2,4)} A^{(\dagger)} A B\right)=u\left(I_{p}-B^{(1,2,4)} A^{(1,3,4)} A B\right)=u\left(I_{p}-B^{(1,2,4)} A^{(1,2,4)} A B\right) \\
& =u\left(I_{p}-B^{(1,2,4)} A^{(1,4)} A B\right)=u\left(I_{p}-B^{(1,4)} A^{\dagger} A B\right)=u\left(I_{p}-B^{(1,4)} A^{(1,3,4)} A B\right) \\
& =u\left(I_{p}-B^{(1,4)} A^{(1,2,4)} A B\right)=u\left(I_{p}-B^{(1,4)} A^{(1,4)} A B\right)=u\left(I_{p}-B^{(1,2)} A^{\dagger} A B\right) \\
& =u\left(I_{p}-B^{(1,2)} A^{(1,3,4)} A B\right)=u\left(I_{p}-B^{(1,2)} A^{(1,2,4)} A B\right)=u\left(I_{p}-B^{(1,2)} A^{(1,4)} A B\right) \\
& =u\left(I_{p}-B^{(1)} A^{\dagger} A B\right)=u\left(I_{p}-B^{(1)} A^{(1,3,4)} A B\right)=u\left(I_{p}-B^{(1)} A^{(1,2,4)} A B\right) \\
& =u\left(I_{p}-B^{(1)} A^{(1,4)} A B\right)=u\left(I_{p}-B^{\dagger} A^{(1,2,3)} A B\right)=u\left(I_{p}-B^{\dagger} A^{(1,3)} A B\right) \\
& =u\left(I_{p}-B^{\dagger} A^{(1,2)} A B\right)=u\left(I_{p}-B^{\dagger} A^{(1)} A B\right)=u\left(I_{p}-B^{(1,3,4)} A^{(1,2,3)} A B\right) \\
& =u\left(I_{p}-B^{(1,3,4)} A^{(1,3)} A B\right)=u\left(I_{p}-B^{(1,3,4)} A^{(1,2)} A B\right)=u\left(I_{p}-B^{(1,3,4)} A^{(1)} A B\right) \\
& =u\left(I_{p}-B^{(1,2,3)} A^{(1,2,3)} A B\right)=u\left(I_{p}-B^{(1,2,3)} A^{(1,3)} A B\right)=u\left(I_{p}-B^{(1,2,3)} A^{(1,2)} A B\right) \\
& =u\left(I_{p}-B^{(1,2,3)} A^{(1)} A B\right)=u\left(I_{p}-B^{(1,3)} A^{(1,2,3)} A B\right)=u\left(I_{p}-B^{(1,3)} A^{(1,3)} A B\right) \\
& =u\left(I_{p}-B^{(1,3)} A^{(1,2)} A B\right)=u\left(I_{p}-B^{(1,3)} A^{(1)} A B\right)=r(N)-r(A)-r(B)+p ;
\end{aligned}
$$

the following 16 maximum rank formulas hold

$$
\begin{aligned}
& u\left(I_{p}-B^{(1,2,4)} A^{(1,2,3)} A B\right)=u\left(I_{p}-B^{(1,2,4)} A^{(1,3)} A B\right)=u\left(I_{p}-B^{(1,2,4)} A^{(1,2)} A B\right) \\
& =u\left(I_{p}-B^{(1,2,4)} A^{(1)} A B\right)=u\left(I_{p}-B^{(1,4)} A^{(1,2,3)} A B\right)=u\left(I_{p}-B^{(1,4)} A^{(1,3)} A B\right) \\
& =u\left(I_{p}-B^{(1,4)} A^{(1,2)} A B\right)=u\left(I_{p}-B^{(1,4)} A^{(1)} A B\right)=u\left(I_{p}-B^{(1,2)} A^{(1,2,3)} A B\right) \\
& =u\left(I_{p}-B^{(1,2)} A^{(1,3)} A B\right)=u\left(I_{p}-B^{(1,2)} A^{(1,2)} A B\right)=u\left(I_{p}-B^{(1,2)} A^{(1)} A B\right) \\
& =u\left(I_{p}-B^{(1)} A^{(1,2,3)} A B\right)=u\left(I_{p}-B^{(1)} A^{(1,3)} A B\right)=u\left(I_{p}-B^{(1)} A^{(1,2)} A B\right) \\
& =u\left(I_{p}-B^{(1)} A^{(1)} A B\right)=\min \{p, \quad n+p-r(A)-r(B)\} .
\end{aligned}
$$

(t) The following 48 minimum rank formulas hold

$$
\begin{aligned}
& v\left(I_{p}-B^{(1,2,4)} A^{\dagger} A B\right)=v\left(I_{p}-B^{(1,2,4)} A^{(1,3,4)} A B\right)=v\left(I_{p}-B^{(1,2,4)} A^{(1,2,4)} A B\right) \\
& =v\left(I_{p}-B^{(1,2,4)} A^{(1,4)} A B\right)=v\left(I_{p}-B^{(1,4)} A^{\dagger} A B\right)=v\left(I_{p}-B^{(1,4)} A^{(1,3,4)} A B\right) \\
& =v\left(I_{p}-B^{(1,4)} A^{(1,2,4)} A B\right)=v\left(I_{p}-B^{(1,4)} A^{(1,4)} A B\right)=v\left(I_{p}-B^{(1,2)} A^{\dagger} A B\right) \\
& =v\left(I_{p}-B^{(1,2)} A^{(1,3,4)} A B\right)=v\left(I_{p}-B^{(1,2)} A^{(1,2,4)} A B\right)=v\left(I_{p}-B^{(1,2)} A^{(1,4)} A B\right) \\
& =v\left(I_{p}-B^{(1)} A^{\dagger} A B\right)=v\left(I_{p}-B^{(1)} A^{(1,3,4)} A B\right)=v\left(I_{p}-B^{(1)} A^{(1,2,4)} A B\right) \\
& =v\left(I_{p}-B^{(1)} A^{(1,4)} A B\right)=v\left(I_{p}-B^{\dagger} A^{(1,2,3)} A B\right)=v\left(I_{p}-B^{\dagger} A^{(1,3)} A B\right)
\end{aligned}
$$




$$
\begin{aligned}
& =v\left(I_{p}-B^{\dagger} A^{(1,2)} A B\right)=v\left(I_{p}-B^{\dagger} A^{(1)} A B\right)=v\left(I_{p}-B^{(1,3,4)} A^{(1,2,3)} A B\right) \\
& =v\left(I_{p}-B^{(1,3,4)} A^{(1,3)} A B\right)=v\left(I_{p}-B^{(1,3,4)} A^{(1,2)} A B\right)=v\left(I_{p}-B^{(1,3,4)} A^{(1)} A B\right) \\
& =v\left(I_{p}-B^{(1,2,3)} A^{(1,2,3)} A B\right)=v\left(I_{p}-B^{(1,2,3)} A^{(1,3)} A B\right)=v\left(I_{p}-B^{(1,2,3)} A^{(1,2)} A B\right) \\
& =v\left(I_{p}-B^{(1,2,3)} A^{(1)} A B\right)=v\left(I_{p}-B^{(1,3)} A^{(1,2,3)} A B\right)=v\left(I_{p}-B^{(1,3)} A^{(1,3)} A B\right) \\
& =v\left(I_{p}-B^{(1,3)} A^{(1,2)} A B\right)=v\left(I_{p}-B^{(1,3)} A^{(1)} A B\right)=v\left(I_{p}-B^{(1,2,4)} A^{(1,2,3)} A B\right) \\
& =v\left(I_{p}-B^{(1,2,4)} A^{(1,3)} A B\right)=v\left(I_{p}-B^{(1,2,4)} A^{(1,2)} A B\right)=v\left(I_{p}-B^{(1,2,4)} A^{(1)} A B\right) \\
& =v\left(I_{p}-B^{(1,4)} A^{(1,2,3)} A B\right)=v\left(I_{p}-B^{(1,4)} A^{(1,3)} A B\right)=v\left(I_{p}-B^{(1,4)} A^{(1,2)} A B\right) \\
& =v\left(I_{p}-B^{(1,4)} A^{(1)} A B\right)=v\left(I_{p}-B^{(1,2)} A^{(1,2,3)} A B\right)=v\left(I_{p}-B^{(1,2)} A^{(1,3)} A B\right) \\
& =v\left(I_{p}-B^{(1,2)} A^{(1,2)} A B\right)=v\left(I_{p}-B^{(1,2)} A^{(1)} A B\right)=v\left(I_{p}-B^{(1)} A^{(1,2,3)} A B\right) \\
& =v\left(I_{p}-B^{(1)} A^{(1,3)} A B\right)=v\left(I_{p}-B^{(1)} A^{(1,2)} A B\right)=v\left(I_{p}-B^{(1)} A^{(1)} A B\right) \\
& =p-r(A B) .
\end{aligned}
$$

(u) The following 64 rank equalities hold for the eight commonly-used types of generalized inverses $A^{(i, . ., j)}$ and $B^{\left(s_{2}, \ldots, t_{2}\right)}$

$$
u\left(A B B^{\left(s_{2}, \ldots, t_{2}\right)} A^{(i, \ldots, j)} A B\right)=r(A B)
$$

(v) The following 32 minimum rank formulas hold

$$
\begin{aligned}
& v\left(A B B^{\dagger} A^{(1,2,3)} A B\right)=v\left(A B B^{\dagger} A^{(1,3)} A B\right)=v\left(A B B^{\dagger} A^{(1,2)} A B\right) \\
& =v\left(B B^{\dagger} A^{(1)} A B\right)=v\left(A B B^{(1,3,4)} A^{(1,2,3)} A B\right)=v\left(A B B^{(1,3,4)} A^{(1,3)} A B\right) \\
& =v\left(A B B^{(1,3,4)} A^{(1,2)} A B\right)=v\left(A B B^{(1,3,4)} A^{(1)} A B\right)=v\left(A B B^{(1,2,3)} A^{(1,2,3)} A B\right) \\
& =v\left(A B B^{(1,2,3)} A^{(1,3)} A B\right)=v\left(A B B^{(1,2,3)} A^{(1,2)} A B\right)=v\left(A B B^{(1,2,3)} A^{(1)} A B\right) \\
& =v\left(A B B^{(1,3)} A^{(1,2,3)} A B\right)=v\left(A B B^{(1,3)} A^{(1,3)} A B\right)=v\left(A B B^{(1,3)} A^{(1,2)} A B\right) \\
& =v\left(A B B^{(1,3)} A^{(1)} A B\right)=v\left(A B B^{(1,2,4)} A^{\dagger} A B\right)=v\left(A B B^{(1,4)} A^{\dagger} A B\right) \\
& =v\left(A B B^{(1,2)} A^{\dagger} A B\right)=v\left(A B B^{(1)} A^{\dagger} A B\right)=v\left(A B B^{(1,2,4)} A^{(1,3,4)} A B\right) \\
& =v\left(A B B^{(1,4)} A^{(1,3,4)} A B\right)=v\left(A B B^{(1,2)} A^{(1,3,4)} A B\right)=v\left(A B B^{(1)} A^{(1,3,4)} A B\right) \\
& =v\left(A B B^{(1,2,4)} A^{(1,2,4)} A B\right)=v\left(A B B^{(1,4)} A^{(1,2,4)} A B\right)=v\left(A B B^{(1,2)} A^{(1,2,4)} A B\right) \\
& =v\left(A B B^{(1)} A^{(1,2,4)} A B\right)=v\left(A B B^{(1,2,4)} A^{(1,4)} A B\right)=v\left(A B B^{(1,4)} A^{(1,4)} A B\right) \\
& =v\left(A B B^{(1,2)} A^{(1,4)} A B\right)=v\left(A B B^{(1)} A^{(1,4)} A B\right)=r(A)+r(B)-r(N) ;
\end{aligned}
$$

the following 16 minimum rank formulas hold

$$
\begin{aligned}
& v\left(A B B^{(1,2,4)} A^{(1,2,3)} A B\right)=v\left(A B B^{(1,2,4)} A^{(1,3)} A B\right)=v\left(A B B^{(1,2,4)} A^{(1,2)} A B\right) \\
& =v\left(A B B^{(1,2,4)} A^{(1)} A B\right)=v\left(A B B^{(1,4)} A^{(1,2,3)} A B\right)=v\left(A B B^{(1,4)} A^{(1,3)} A B\right) \\
& =v\left(A B B^{(1,4)} A^{(1,2)} A B\right)=v\left(A B B^{(1,4)} A^{(1)} A B\right)=v\left(A B B^{(1,2)} A^{(1,2,3)} A B\right) \\
& =v\left(A B B^{(1,2)} A^{(1,3)} A B\right)=v\left(A B B^{(1,2)} A^{(1,2)} A B\right)=v\left(A B B^{(1,2)} A^{(1)} A B\right) \\
& =v\left(A B B^{(1)} A^{(1,2,3)} A B\right)=v\left(A B B^{(1)} A^{(1,3)} A B\right)=v\left(A B B^{(1)} A^{(1,2)} A B\right) \\
& =v\left(A B B^{(1)} A^{(1)} A B\right)=\max \{0, \quad r(A)+r(B)-n\} .
\end{aligned}
$$

In addition, we shall use the following rank and range formulas for various products of two matrices.

Lemma 8.5 ( $[86,87])$. Let $A \in \mathbb{C}^{m \times n}$ and $B \in \mathbb{C}^{m \times p}$, and $P \in \mathbb{C}^{p \times m}$. Then the following results hold

$$
\begin{aligned}
& r\left(A A^{*} A B B^{*} B\right)=r\left(A^{*} A B B^{*}\right)=r\left(A B B^{*} A^{*}\right)=r\left(B^{*} A^{*} A B\right)=r(A B), \\
& r\left[\left(A^{*} A\right)^{1 / 2}\left(B B^{*}\right)^{1 / 2}\right]=r\left[\left(B B^{*}\right)^{1 / 2}\left(A^{*} A\right)^{1 / 2}\right]=r(A B), \\
& r\left(B^{\dagger} A^{\dagger}\right)=r\left(B^{*} A^{\dagger}\right)=r\left(B^{\dagger} A^{*}\right)=r(A B),
\end{aligned}
$$




$$
\begin{aligned}
& r\left(A^{\dagger} A B B^{\dagger}\right)=r\left(A^{\dagger} A B B^{*}\right)=r\left(A^{*} A B B^{\dagger}\right)=r(A B), \\
& r\left(B B^{\dagger} A^{\dagger} A\right)=r\left(B B^{\dagger} A^{*} A\right)=r\left(B B^{*} A^{\dagger} A\right)=r(A B), \\
& r\left(A B B^{\dagger} A^{\dagger}\right)=r\left(A B B^{\dagger} A^{*}\right)=r\left(A B B^{*} A^{\dagger}\right)=r(A B), \\
& r\left(B^{\dagger} A^{\dagger} A B\right)=r\left(B^{\dagger} A^{*} A B\right)=r\left(B^{*} A^{\dagger} A B\right)=r(A B), \\
& r\left(A B B^{\dagger} A^{\dagger} A B\right)=r\left(A B B^{\dagger} A^{*} A B\right)=r\left(A B B^{*} A^{\dagger} A B\right)=r(A B), \\
& r\left(B^{\dagger} A^{\dagger} A B B^{\dagger} A^{\dagger}\right)=r\left(B^{\dagger} A^{*} A B B^{\dagger} A^{\dagger}\right)=r\left(B^{\dagger} A^{\dagger} A B B^{*} A^{\dagger}\right)=r(A B), \\
& \left.\left.r\left[\left(B B^{*}\right)^{\dagger}\left(A^{*} A\right)^{\dagger}\right)\right]=r\left[\left(B B^{*}\right)^{\dagger}\left(A^{*} A\right)\right]=r\left[\left(B B^{*}\right)\left(A^{*} A\right)^{\dagger}\right)\right]=r(A B), \\
& \left.\left.r\left[B^{\dagger}\left(A^{*} A\right)^{\dagger}\right)\right]=r\left(B^{\dagger} A^{*} A\right)=r\left[B^{*}\left(A^{*} A\right)^{\dagger}\right)\right]=r(A B), \\
& r\left[\left(B B^{*}\right)^{\dagger} A^{\dagger}\right]=r\left[\left(B B^{*}\right)^{\dagger} A^{*}\right]=r\left(B B^{*} A^{\dagger}\right)=r(A B), \\
& r\left[\left(A^{\dagger}\right)^{*}\left(B^{\dagger}\right)^{*}\right]=r\left[\left(A^{\dagger}\right)^{*} B\right]=r\left[A\left(B^{\dagger}\right)^{*}\right]=r(A B),
\end{aligned}
$$

and

$$
\begin{aligned}
& \text { both } \mathscr{R}(A) \subseteq \mathscr{R}(B) \text { and } r(A)=r(B) \Rightarrow \mathscr{R}(A)=\mathscr{R}(B), \\
& \mathscr{R}(A) \subseteq \mathscr{R}(B) \Rightarrow \mathscr{R}(P A) \subseteq \mathscr{R}(P B), \\
& \mathscr{R}(A)=\mathscr{R}(B) \Rightarrow \mathscr{R}(P A)=\mathscr{R}(P B), \\
& \mathscr{R}\left(A B B^{*} A^{*}\right)=\mathscr{R}\left(A B B^{*}\right)=\mathscr{R}(A B), \\
& \mathscr{R}\left(B^{*} A^{*} A B\right)=\mathscr{R}\left(B^{*} A^{*} A\right)=\mathscr{R}\left(B^{*} A^{*}\right), \\
& \mathscr{R}\left(A B B^{\dagger} A^{\dagger} A B\right)=\mathscr{R}\left(A B B^{\dagger} A^{\dagger}\right)=\mathscr{R}(A B), \\
& \mathscr{R}\left(B^{\dagger} A^{\dagger} A B B^{\dagger} A^{\dagger}\right)=\mathscr{R}\left(B^{\dagger} A^{\dagger} A B\right)=\mathscr{R}\left(B^{\dagger} A^{\dagger}\right)=\mathscr{R}\left(B^{\dagger} A^{*}\right) .
\end{aligned}
$$

Eqs. 8.5- 8.24 can be used to establish various formulas for calculating the ranks of matrix expressions or matrix-valued functions composed by products of two matrices with their conjugates and Moore-Penrose inverses, which we can use, as demonstrated below, to describe performance and reveal fundamental properties of the matrix expressions and matrix-valued functions. Applying the formulas in Section 4 to these matrix-valued functions, we obtain the following rank formulas.

Theorem 8.6. Let $A \in \mathbb{C}^{m \times n}$ and $B \in \mathbb{C}^{n \times p}$, and denote $M=A B, H=A B B^{\dagger} A^{\dagger} A B$, and $N=\left[A^{*}, B\right]$.

(a) The following rank formulas

$$
\begin{aligned}
& r\left(M-M B^{\dagger} A^{(1,3,4)} M\right)=r\left(M-M B^{\dagger} A^{(1,2,4)} M\right)=r\left(M-M B^{\dagger} A^{(1,4)} M\right) \\
& =r\left(M-M B^{(1,3,4)} A^{\dagger} M=r\left(M-M B^{(1,3,4)} A^{(1,3,4)} M\right)=r\left(M-M B^{(1,3,4)} A^{(1,2,4)} M\right)\right. \\
& =r\left(M-M B^{(1,3,4)} A^{(1,4)} M\right)=r\left(M-M B^{(1,2,3)} A^{\dagger} M\right)=r\left(M-M B^{(1,2,3)} A^{(1,3,4)} M\right) \\
& =r\left(M-M B^{(1,2,3)} A^{(1,2,4)} M\right)=r\left(M-M B^{(1,2,3)} A^{(1,4)} M\right)=r\left(M-M B^{(1,3)} A^{\dagger} M\right) \\
& =r\left(M-M B^{(1,3)} A^{(1,3,4)} M\right)=r\left(M-M B^{(1,3)} A^{(1,2,4)} M\right)=r\left(M-M B^{(1,3)} A^{(1,4)} M\right) \\
& =r\left(M-M B^{\dagger} A^{\dagger} M\right)=r\left(A E_{B} F_{A} B\right)=r(N)+r(A B)-r(A)-r(B)
\end{aligned}
$$

hold for all $A^{\left(s_{1}, \ldots, t_{1}\right)}$ and $B^{\left(s_{2}, \ldots, t_{2}\right)}$ in them.

(b) The following rank formulas hold

$$
\begin{aligned}
& \max _{A^{(1,2,3)}} r\left(M-M B^{\dagger} A^{(1,2,3)} M\right)=\max _{A^{(1,3)}} r\left(M-M B^{\dagger} A^{(1,3)} M\right) \\
& =\max _{A^{(1,2)}} r\left(M-M B^{\dagger} A^{(1,2)} M\right)=\max _{B^{(1)}} r\left(M-M B^{\dagger} A^{(1)} M\right) \\
& =\max _{B^{(1,3,4)}, A^{(1,2,3)}} r\left(M-M B^{(1,3,4)} A^{(1,2,3)} M\right)=\max _{B^{(1,3,4)}, A^{(1,3)}} r\left(M-M B^{(1,3,4)} A^{(1,3)} M\right) \\
& =\max _{B^{(1,3,4)}, A^{(1,2)}} r\left(M-M B^{(1,3,4)} A^{(1,2)} M\right)=\max _{B^{(1,3,4)}, A^{(1)}} r\left(M-M B^{(1,3,4)} A^{(1)} M\right) \\
& =\max _{B^{(1,2,3)}, A^{(1,2,3)}} r\left(M-M B^{(1,2,3)} A^{(1,2,3)} M\right)=\max _{B^{(1,2,3)}, A^{(1,3)}} r\left(M-M B^{(1,2,3)} A^{(1,3)} M\right) \\
& =\max _{B^{(1,2,3)}, A^{(1,2)}} r\left(M-M B^{(1,2,3)} A^{(1,2)} M\right)=\max _{B^{(1,2,3)}, A^{(1)}} r\left(M-M B^{(1,2,3)} A^{(1)} M\right)
\end{aligned}
$$




$$
\begin{aligned}
& =\max _{B^{(1,3)}, A^{(1,2,3)}} r\left(M-M B^{(1,3)} A^{(1,2,3)} M\right)=\max _{B^{(1,3)}, A^{(1,3)}} r\left(M-M B^{(1,3)} A^{(1,3)} M\right) \\
& =\max _{B^{(1,3)}, A^{(1,2)}} r\left(M-M B^{(1,3)} A^{(1,2)} M\right)=\max _{B^{(1,3)}, A^{(1)}} r\left(M-M B^{(1,3)} A^{(1)} M\right) \\
& =r(N)+r(M)-r(A)-r(B) .
\end{aligned}
$$

(c) The following rank formulas hold

$$
\begin{aligned}
& \max _{B^{(1,2,4)}} r\left(M-M B^{(1,2,4)} A^{\dagger} M\right)=\max _{B^{(1,2,4)}, A^{(1,3,4)}} r\left(M-M B^{(1,2,4)} A^{(1,3,4)} M\right) \\
= & \max _{B^{(1,2,4)}, A^{(1,2,4)}} r\left(M-M B^{(1,2,4)} A^{(1,2,4)} M=\max _{B^{(1,2,3)}, A^{(1,4)}} r\left(M-M B^{(1,2,4)} A^{(1,4)} M\right)\right. \\
= & \max _{B^{(1,4)}} r\left(M-M B^{(1,4)} A^{\dagger} M\right)=\max _{B^{(1,4)}, A^{(1,3,4)}} r\left(M-M B^{(1,4)} A^{(1,3,4)} M\right) \\
= & \max _{B^{(1,4)}, A^{(1,2,4)}} r\left(M-M B^{(1,4)} A^{(1,2,4)} M\right)=\max _{B^{(1,4)}, A^{(1,4)}} r\left(M-M B^{(1,4)} A^{(1,4)} M\right) \\
= & \max _{B^{(1,2)}} r\left(M-M B^{(1,2)} A^{\dagger} M\right)=\max _{B^{(1,4)}, A^{(1,2,4)}} r\left(M-M B^{(1,2)} A^{(1,3,4)} M\right) \\
= & \max _{B^{(1,2)}, A^{(1,2,4)}} r\left(M-M B^{(1,2)} A^{(1,2,4)} M\right)=\max _{B^{(1,2)}, A^{(1,4)}} r\left(M-M B^{(1,2)} A^{(1,4)} M\right) \\
= & \max _{B^{(1)}} r\left(M-M B^{(1)} A^{\dagger} M\right)=\max _{B^{(1)}, A^{(1,3,4)}} r\left(M-M B^{(1)} A^{(1,3,4)} M\right) \\
= & \max _{B^{(1)}, A^{(1,2,4)}} r\left(M-M B^{(1)} A^{(1,2,4)} M=\max _{B^{(1)}, A^{(1,4)}} r\left(M-M B^{(1)} A^{(1,4)} M\right)\right. \\
= & r(N)+r(M)-r(A)-r(B) .
\end{aligned}
$$

(d) The following rank formulas hold

$$
\begin{aligned}
& \max _{A^{(1,2,3)}} r\left(H-M B^{\dagger} A^{(1,2,3)} M\right)=\max _{A^{(1,3)}} r\left(H-M B^{\dagger} A^{(1,3)} M\right) \\
= & \max _{A^{(1,2)}} r\left(H-M B^{\dagger} A^{(1,2)} M\right)=\max _{B^{(1)}} r\left(H-M B^{\dagger} A^{(1)} M\right) \\
= & \max _{B^{(1,3,4)}, A^{(1,2,3)}} r\left(H-M B^{(1,3,4)} A^{(1,2,3)} M\right)=\max _{B^{(1,3,4)}, A^{(1,3)}} r\left(H-M B^{(1,3,4)} A^{(1,3)} M\right) \\
= & \max _{B^{(1,3,4)}, A^{(1,2)}} r\left(H-M B^{(1,3,4)} A^{(1,2)} M\right)=\max _{B^{(1,3,4)}, A^{(1)}} r\left(M B^{\dagger} A^{\dagger} M y-M B^{(1,3,4)} A^{(1)} M\right) \\
= & \max _{B^{(1,2,3)}, A^{(1,2,3)}} r\left(H-M B^{(1,2,3)} A^{(1,2,3)} M\right)=\max _{B^{(1,2,3)}, A^{(1,3)}} r\left(H-M B^{(1,2,3)} A^{(1,3)} M\right) \\
= & \max _{B^{(1,2,3)}, A^{(1,2)}} r\left(H-M B^{(1,2,3)} A^{(1,2)} M\right)=\max _{B^{(1,2,3)}, A^{(1)}}^{r}\left(H-M B^{(1,2,3)} A^{(1)} M\right. \\
= & \max _{B^{(1,3)}, A^{(1,2,3)}} r\left(H-M B^{(1,3)} A^{(1,2,3)} M\right)=\max _{B^{(1,3)}, A^{(1,3)}}^{r}\left(M B^{\dagger} A^{\dagger} M y-M B^{(1,3)} A^{(1,3)} M\right) \\
= & \max _{B^{(1,3)}, A^{(1,2)}} r\left(H-M B^{(1,3)} A^{(1,2)} M\right)=\max _{B^{(1,3)}, A^{(1)}}^{r}\left(H-M B^{(1,3)} A^{(1)} M\right) \\
= & r(N)+r(M)-r(A)-r(B) .
\end{aligned}
$$

(e) The following rank formulas hold

$$
\begin{aligned}
& \max _{B^{(1,2,4)}}\left(H-M B^{(1,2,4)} A^{\dagger} M\right)=\max _{B^{(1,2,4)}, A^{(1,3,4)}} r\left(H-M B^{(1,2,4)} A^{(1,3,4)} M\right) \\
= & \max _{B^{(1,2,4)}, A^{(1,2,4)}} r\left(H-M B^{(1,2,4)} A^{(1,2,4)} M=\max _{B^{(1,2,3)}, A^{(1,4)}} r\left(H-M B^{(1,2,4)} A^{(1,4)} M\right)\right. \\
= & \max _{B^{(1,4)}} r\left(H-M B^{(1,4)} A^{\dagger} M\right)=\max _{B^{(1,4)}, A^{(1,3,4)}}^{r}\left(H-M B^{(1,4)} A^{(1,3,4)} M\right) \\
= & \max _{B^{(1,4)}, A(1,2,4)} r\left(H-M B^{(1,4)} A^{(1,2,4)} M\right)=\max _{B^{(1,4)}, A^{(1,4)}} r\left(H-M B^{(1,4)} A^{(1,4)} M\right) \\
= & \max _{B^{(1,2)}}\left(H-M B^{(1,2)} A^{\dagger} M\right)=\max _{B^{(1,4)}, A^{(1,2,4)}}^{r}\left(H-M B^{(1,2)} A^{(1,3,4)} M\right) \\
= & \max _{B^{(1,2)}, A^{(1,2,4)}}\left(H-M B^{(1,2)} A^{(1,2,4)} M\right)=\underset{B^{(1,2)}, A^{(1,4)}}{r}\left(H-M B^{(1,2)} A^{(1,4)} M\right) \\
= & \max _{B^{(1)}} r\left(H-M B^{(1)} A^{\dagger} M\right)=\max _{B^{(1)}, A^{(1,3,4)}}\left(H-M B^{(1)} A^{(1,3,4)} M\right)
\end{aligned}
$$




$$
\begin{aligned}
& =\max _{B^{(1)}, A^{(1,2,4)}} r\left(H-M B^{(1)} A^{(1,2,4)} M=\max _{B^{(1)}, A^{(1,4)}} r\left(H-M B^{(1)} A^{(1,4)} M\right)\right. \\
& =r(N)+r(M)-r(A)-r(B) .
\end{aligned}
$$

(f) The following rank formulas hold

$$
\begin{aligned}
& \max _{A^{(1,2,3)}, B^{(1,2,4)}} r\left(M-M B^{(1,2,4)} A^{(1,2,3)} M\right)=\max _{A^{(1,3)}, B^{(1,2,4)}} r\left(M-M B^{(1,2,4)} A^{(1,3)} M\right) \\
= & \max _{A^{(1,2)}, B^{(1,2,4)}} r\left(M-M B^{(1,2,4)} A^{(1,2)} M\right)=\max _{A^{(1)}, B^{(1,2,4)}} r\left(M-M B^{(1,2,4)} A^{(1)} M\right) \\
= & \max _{A^{(1,2,3)}, B^{(1,4)}} r\left(M-M B^{(1,4)} A^{(1,2,3)} M\right)=\max _{A^{(1,3)}, B^{(1,4)}} r\left(M-M B^{(1,4)} A^{(1,3)} M\right) \\
= & \max _{A^{(1,2)}, B^{(1,4)}} r\left(M-M B^{(1,4)} A^{(1,2)} M\right)=\max _{A^{(1)}, B^{(1,4)}} r\left(M-M B^{(1,4)} A^{(1)} M\right) \\
= & \max _{A^{(1,2,3)}, B^{(1,2)}} r\left(M-M B^{(1,2)} A^{(1,2,3)} M\right)=\max _{A^{(1,3)}, B^{(1,2)}} r\left(M-M B^{(1,2)} A^{(1,3)} M\right) \\
= & \max _{A^{(1,2)}, B^{(1,2)}} r\left(M-M B^{(1,2)} A^{(1,2)} M\right)=\max _{A^{(1)}, B^{(1,2)}} r\left(M-M B^{(1,2)} A^{(1)} M\right) \\
= & \max _{A^{(1,2,3)}, B^{(1)}} r\left(M-M B^{(1)} A^{(1,2,3)} M\right)=\max _{A^{(1,3)}, B^{(1)}} r\left(M-M B^{(1)} A^{(1,3)} M\right) \\
= & \max _{A^{(1,2)}, B^{(1)}} r\left(M-M B^{(1)} A^{(1,2)} M\right)=\max _{A^{(1)}, B^{(1)}} r\left(M-M B^{(1)} A^{(1)} M\right) \\
= & \min \{r(M), r(M)-r(A)-r(B)+n\}=\min _{n}\left\{r(M), r\left(F_{A} E_{B}\right)\right\} .
\end{aligned}
$$

(g) The following rank formulas hold

$$
\begin{aligned}
& \max _{A^{(1,2,3)}, B^{(1,2,4)}} r\left(H-M B^{(1,2,4)} A^{(1,2,3)} M\right)=\max _{A^{(1,3)}, B^{(1,2,4)}} r\left(H-M B^{(1,2,4)} A^{(1,3)} M\right) \\
= & \max _{A^{(1,2)}, B^{(1,2,4)}} r\left(H-M B^{(1,2,4)} A^{(1,2)} M\right)=\max _{A^{(1)}, B^{(1,2,4)}} r\left(H-M B^{(1,2,4)} A^{(1)} M\right) \\
= & \max _{A^{(1,2,3)}, B^{(1,4)}} r\left(H-M B^{(1,4)} A^{(1,2,3)} M\right)=\max _{A^{(1,3)}, B^{(1,4)}} r\left(H-M B^{(1,4)} A^{(1,3)} M\right) \\
= & \max _{A^{(1,2)}, B^{(1,4)}} r\left(H-M B^{(1,4)} A^{(1,2)} M\right)=\max _{A^{(1)}, B^{(1,4)}} r\left(H-M B^{(1,4)} A^{(1)} M\right) \\
= & \max _{A^{(1,2,3)}, B^{(1,2)}} r\left(H-M B^{(1,2)} A^{(1,2,3)} M\right)=\max _{A^{(1,3)}, B^{(1,2)}} r\left(H-M B^{(1,2)} A^{(1,3)} M\right) \\
= & \max _{A^{(1,2)}, B^{(1,2)}} r\left(H-M B^{(1,2)} A^{(1,2)} M\right)=\max _{A^{(1)}, B^{(1,2)}} r\left(H-M B^{(1,2)} A^{(1)} M\right) \\
= & \max _{A^{(1,2,3)}, B^{(1)}} r\left(H-M B^{(1)} A^{(1,2,3)} M\right)=\max _{A^{(1,3)}, B^{(1)}} r\left(H-M B^{(1)} A^{(1,3)} M\right) \\
= & \max _{A^{(1,2)}, B^{(1)}} r\left(H-M B^{(1)} A^{(1,2)} M\right)=\max _{A^{(1)}, B^{(1)}} r\left(H-M B^{(1)} A^{(1)} M\right) \\
= & \left.\min _{\min } r(M), r(M)-r(A)-r(B)+n\right\}=\min \left\{r(M), r\left(F_{A} E_{B}\right)\right\} . ?
\end{aligned}
$$

Proof. It is easy to verify that $A E_{B} F_{A} B=A\left(I_{n}-B B^{\dagger}-A^{\dagger} A+B B^{\dagger} A^{\dagger} A\right) B=M B^{\dagger} A^{\dagger} M-M$. The last rank formula in 8.25 was established in 8]. We next give a direct proof of the last rank formula in (8.25). Applying (4.6) and 8.2 to $M-M B^{\dagger} A^{\dagger} M$ gives

$$
\begin{aligned}
r\left(M-M B^{\dagger} A^{\dagger} M\right) & =r\left(A B+A B P J^{\dagger} Q A B\right) \\
& =r\left[\begin{array}{ccc}
B^{*} A^{*} & B^{*} B B^{*} & 0 \\
A^{*} A A^{*} & 0 & A^{*} A B \\
0 & A B B^{*} & -A B
\end{array}\right]-r(A)-r(B) \\
& =r\left[\begin{array}{ccc}
B^{*} A^{*} & B^{*} B & 0 \\
A A^{*} & 0 & A B \\
0 & A B & -A B
\end{array}\right]-r(A)-r(B) \\
& =r\left[\begin{array}{ccc}
B^{*} A^{*} & B^{*} B & 0 \\
A A^{*} & A B & 0 \\
0 & 0 & -A B
\end{array}\right]-r(A)-r(B) \\
& =r\left(\left[A^{*}, B\right]^{*}\left[A^{*}, B\right]\right)+r(A B)-r(A)-r(B) \\
& =r(N)+r(A B)-r(A)-r(B),
\end{aligned}
$$


thus establishing the last two rank formulas in (8.25). The first 16 rank equalities in (8.25) follow directly from 3.152 .

Applying (4.27) to the difference of $A B$ and (3.153) gives

$$
\max _{U} r\left(A B-H-A B B^{\dagger} F_{A} U A B\right)=\min \left\{r\left[A B-H, A B B^{\dagger} F_{A}\right], \quad r(A B)\right\},
$$

where by (4.2) and 4.3,

$$
\begin{aligned}
r\left[A B-H, A B B^{\dagger} F_{A}\right] & =r\left[\begin{array}{cc}
A B-H & A B B^{\dagger} \\
0 & A
\end{array}\right]-r(A) \\
& =r\left[\begin{array}{cc}
A B & A B B^{\dagger} \\
A B & A
\end{array}\right]-r(A) \\
& =r\left[\begin{array}{cc}
A B & 0 \\
0 & A E_{B}
\end{array}\right]-r(A)=r(A B)+r\left(E_{B} A^{*}\right)-r(A) \\
& =r(N)+r(A B)-r(A)-r(B) .
\end{aligned}
$$

Substituting 8.34 into 8.33 and noticing that $r(N)+r(A B)-r(A)-r(B) \leqslant r(A B)$, we obtain 8.26). Applying (4.27) to the difference of $A B$ and (3.154), we are also able to obtain

$$
\max _{V} r\left(A B-H-A B V E_{B} A^{\dagger} A B\right)=r(N)+r(A B)-r(A)-r(B),
$$

as required for 8.27). Applying (4.27) to $A B B^{\dagger} F_{A} U A B$ and $A B V E_{B} A^{\dagger} A B \mathrm{~m}$ and simplifying by (4.2) and (4.3) we obtain the following two rank formulas

$$
\begin{aligned}
& \max _{U} r\left(A B B^{\dagger} F_{A} U A B\right)=r\left(A B B^{\dagger} F_{A}\right)=r(N)+r(A B)-r(A)-r(B), \\
& \max _{V} r\left(A B V E_{B} A^{\dagger} A B\right)=r\left(E_{B} A^{\dagger} A B\right)=r(N)+r(A B)-r(A)-r(B),
\end{aligned}
$$

thus establishing 8.28 and 8.29 .

A common feature of (8.25)-(8.29) is in that all the terms on the right-hand sides of these formulas are identical. Thus, setting all sides of these formulas equal to zero will produce many equivalent facts concerning the matrix operations on the left-hand sides, which will be presented in the following sections in the classification study of ROLs.

\section{Set inclusions for $\{1\}-,\{1,2\}-,\{1,3\}-$, and $\{1,4\}$-generalized inverses of $A B$}

Several cases of the set inclusions in (1.9) were considered, such as,

$$
\begin{gathered}
\left\{(A B)^{(1)}\right\} \supseteq\left\{B^{1)} A^{(1)}\right\}, \quad\left\{(A B)^{(1,2)}\right\} \supseteq\left\{B^{1,2)} A^{(1,2)}\right\}, \quad\left\{(A B)^{(1,3)}\right\} \supseteq\left\{B^{1,3)} A^{(1,3)}\right\}, \\
\left\{(A B)^{(1,4)}\right\} \supseteq\left\{B^{1,4)} A^{(1,4)}\right\}, \quad\left\{(A B)^{(1,2,3)}\right\} \supseteq\left\{B^{1,2,3)} A^{(1,2,3)}\right\}, \quad\left\{(A B)^{(1,2,4)}\right\} \supseteq\left\{B^{1,2,4)} A^{(1,2,4)}\right\},
\end{gathered}
$$

in the literature in the past decades, see e,g., $21,22,44,47,48,56,71,75,85,106,108,110,114,115]$. Despite the fact that ROLs have been around for a long time, there are still many open problems regarding ROLs under various assumptions. In this and next sections, we present various known and novel results on the 512 set inclusions in (1.9).

For the first case $(A B)^{(1)}$, we see by definition that

$$
\left\{(A B)^{(1)}\right\} \supseteq\left\{B^{\left(s_{2}, \ldots, t_{2}\right)} A^{\left(s_{1}, \ldots, t_{1}\right)}\right\} \Leftrightarrow A B B^{\left(s_{2}, \ldots, t_{2}\right)} A^{\left(s_{1}, \ldots, t_{1}\right)} A B=A B
$$

hold for the eight commonly-used types of generalized inverses $A^{\left(s_{1}, \ldots, t_{1}\right)}$ and $B^{\left(s_{2}, \ldots, t_{2}\right)}$, respectively. In this situation, substituting (3.152)-(3.155) into (9.1), we see that the equalities in (9.1) are converted to 63 linear or multilinear matrix equations with some unknown matrices for the eight commonly-used types of generalized inverses of $A$ and $B$ except $A B B^{\dagger} A^{\dagger} A B=A B$. Our first group of results describe various equivalent statements for the 64 ROLs in (9.1) to hold, which correspond in turn to the statistical fact in (2.27). 
Theorem 9.1. Let $A \in \mathbb{C}^{m \times n}$ and $B \in \mathbb{C}^{n \times p}$. Then the following 165 statements are equivalent:

\begin{tabular}{|c|c|c|c|}
\hline$\langle 1\rangle$ & $\left\{(A B)^{(1)}\right\} \supseteq\left\{B^{\dagger} A^{(1)}\right\}$. & $\langle 2\rangle$ & $\left\{(A B)^{(1)}\right\} \supseteq\left\{B^{\dagger} A^{(1,2)}\right\}$. \\
\hline$\langle 3\rangle$ & $\left\{(A B)^{(1)}\right\} \supseteq\left\{B^{\dagger} A^{(1,3)}\right\}$. & $\langle 4\rangle$ & $\left\{(A B)^{(1)}\right\} \supseteq\left\{B^{\dagger} A^{(1,4)}\right\}$. \\
\hline$\langle 5\rangle$ & $\left\{(A B)^{(1)}\right\} \supseteq\left\{B^{\dagger} A^{(1,2,3)}\right\}$. & $\langle 6\rangle$ & $\left\{(A B)^{(1)}\right\} \supseteq\left\{B^{\dagger} A^{(1,2,4)}\right\}$. \\
\hline$\langle 7\rangle$ & $\left\{(A B)^{(1)}\right\} \supseteq\left\{B^{\dagger} A^{(1,3,4)}\right\}$. & $\langle 8\rangle$ & $\left\{(A B)^{(1)}\right\} \supseteq\left\{B^{(1,3,4)} A^{(1)}\right\}$ \\
\hline$\langle 9\rangle$ & $\left\{(A B)^{(1)}\right\} \supseteq\left\{B^{(1,3,4)} A^{(1,2)}\right\}$. & $\langle 10\rangle$ & $\left\{(A B)^{(1)}\right\} \supseteq\left\{B^{(1,3,4)} A^{(1,3)}\right\}$. \\
\hline$\langle 11\rangle$ & $\left\{(A B)^{(1)}\right\} \supseteq\left\{B^{(1,3,4)} A^{(1,4)}\right\}$. & $\langle 12\rangle$ & $\left\{(A B)^{(1)}\right\} \supseteq\left\{B^{(1,3,4)} A^{(1,2,3)}\right\}$. \\
\hline$\langle 13\rangle$ & $\left\{(A B)^{(1)}\right\} \supseteq\left\{B^{(1,3,4)} A^{(1,2,4)}\right\}$. & $\langle 14\rangle$ & $\left\{(A B)^{(1)}\right\} \supseteq\left\{B^{(1,3,4)} A^{(1,3,4)}\right\}$. \\
\hline$\langle 15\rangle$ & $\left\{(A B)^{(1)}\right\} \supseteq\left\{B^{(1,3,4)} A^{\dagger}\right\}$ & $\langle 16\rangle$ & $\left\{(A B)^{(1)}\right\} \supseteq\left\{B^{(1,2,4)} A^{(1,4)}\right\}$ \\
\hline$\langle 17\rangle$ & $\left\{(A B)^{(1)}\right\} \supseteq\left\{B^{(1,2,4)} A^{(1,2,4)}\right\}$. & $\langle 18\rangle$ & $\left\{(A B)^{(1)}\right\} \supseteq\left\{B^{(1,2,4)} A^{(1,3,4)}\right\}$. \\
\hline$\langle 19\rangle$ & $\left\{(A B)^{(1)}\right\} \supseteq\left\{B^{(1,2,4)} A^{\dagger}\right\}$ & $\langle 20\rangle$ & $\left\{(A B)^{(1)}\right\} \supseteq\left\{B^{(1,2,3)} A^{(1)}\right\}$ \\
\hline$\langle 21\rangle$ & $\left\{(A B)^{(1)}\right\} \supseteq\left\{B^{(1,2,3)} A^{(1,2)}\right\}$. & $\langle 22\rangle$ & $\left\{(A B)^{(1)}\right\} \supseteq\left\{B^{(1,2,3)} A^{(1,3)}\right\}$. \\
\hline$\langle 23\rangle$ & $\left\{(A B)^{(1)}\right\} \supseteq\left\{B^{(1,2,3)} A^{(1,4)}\right\}$ & $\langle 24\rangle$ & $\left\{(A B)^{(1)}\right\} \supseteq\left\{B^{(1,2,3)} A^{(1,2,3)}\right\}$. \\
\hline$\langle 25\rangle$ & $\left\{(A B)^{(1)}\right\} \supseteq\left\{B^{(1,2,3)} A^{(1,2,4)}\right\}$. & $\langle 26\rangle$ & $\left\{(A B)^{(1)}\right\} \supseteq\left\{B^{(1,2,3)} A^{(1,3,4)}\right\}$. \\
\hline$\langle 27\rangle$ & $\left\{(A B)^{(1)}\right\} \supseteq\left\{B^{(1,2,3)} A^{\dagger}\right\}$ & $\langle 28\rangle$ & $\left\{(A B)^{(1)}\right\} \supseteq\left\{B^{(1,4)} A^{(1,4)}\right\}$ \\
\hline$\langle 29\rangle$ & $\left\{(A B)^{(1)}\right\} \supseteq\left\{B^{(1,4)} A^{(1,2,4)}\right\}$. & $\langle 30\rangle$ & $\left\{(A B)^{(1)}\right\} \supseteq\left\{B^{(1,4)} A^{(1,3,4)}\right\}$. \\
\hline$\langle 31\rangle$ & $\left\{(A B)^{(1)}\right\} \supseteq\left\{B^{(1,4)} A^{\dagger}\right\}$ & $\langle 32\rangle$ & $\left\{(A B)^{(1)}\right\} \supseteq\left\{B^{(1,3)} A^{(1)}\right\}$. \\
\hline$\langle 33\rangle$ & $\left\{(A B)^{(1)}\right\} \supseteq\left\{B^{(1,3)} A^{(1,2)}\right\}$. & $\langle 34\rangle$ & $\left\{(A B)^{(1)}\right\} \supseteq\left\{B^{(1,3)} A^{(1,3)}\right\}$. \\
\hline$\langle 35\rangle$ & $\left\{(A B)^{(1)}\right\} \supseteq\left\{B^{(1,3)} A^{(1,4)}\right\}$. & $\langle 36\rangle$ & $\left\{(A B)^{(1)}\right\} \supseteq\left\{B^{(1,3)} A^{(1,2,3)}\right\}$. \\
\hline$\langle 37\rangle$ & $\left\{(A B)^{(1)}\right\} \supseteq\left\{B^{(1,3)} A^{(1,2,4)}\right\}$. & $\langle 38\rangle$ & $\left\{(A B)^{(1)}\right\} \supseteq\left\{B^{(1,3)} A^{(1,3,4)}\right\}$. \\
\hline$\langle 39\rangle$ & $\left\{(A B)^{(1)}\right\} \supseteq\left\{B^{(1,3)} A^{\dagger}\right\}$ & $\langle 40\rangle$ & $\left\{(A B)^{(1)}\right\} \supseteq\left\{B^{(1,2)} A^{(1,4)}\right\}$. \\
\hline$\langle 41\rangle$ & $\left\{(A B)^{(1)}\right\} \supseteq\left\{B^{(1,2)} A^{(1,2,4)}\right\}$. & $\langle 42\rangle$ & $\left\{(A B)^{(1)}\right\} \supseteq\left\{B^{(1,2)} A^{(1,3,4)}\right\}$. \\
\hline$\langle 43\rangle$ & $\left\{(A B)^{(1)}\right\} \supseteq\left\{B^{(1,2)} A^{\dagger}\right\}$ & $\langle 44\rangle$ & $\left\{(A B)^{(1)}\right\} \supseteq\left\{B^{(1)} A^{(1,4)}\right\}$ \\
\hline$\langle 45\rangle$ & $\left\{(A B)^{(1)}\right\} \supseteq\left\{B^{(1)} A^{(1,2,4)}\right\}$. & $\langle 46\rangle$ & $\left\{(A B)^{(1)}\right\} \supseteq\left\{B^{(1)} A^{(1,3,4)}\right\}$. \\
\hline$\langle 47\rangle$ & $\left\{(A B)^{(1)}\right\} \supseteq\left\{B^{(1)} A^{\dagger}\right\}$ & $\langle 48\rangle$ & $\left\{(A B)^{(1)}\right\} \ni B^{\dagger} A^{\dagger}$ \\
\hline$\langle 49\rangle$ & $\left\{A B(A B)^{(1)}\right\} \supseteq\left\{A B B^{\dagger} A^{(1)}\right\}$. & $\langle 50\rangle$ & $\left\{A B(A B)^{(1)}\right\} \supseteq\left\{A B B^{\dagger} A^{(1,2)}\right\}$. \\
\hline$\langle 51\rangle$ & $\left\{A B(A B)^{(1)}\right\} \supseteq\left\{A B B^{\dagger} A^{(1,3)}\right\}$. & $\langle 52\rangle$ & $\left\{A B(A B)^{(1)}\right\} \supseteq\left\{A B B^{\dagger} A^{(1,4)}\right\}$ \\
\hline$\langle 53\rangle$ & $\left\{A B(A B)^{(1)}\right\} \supseteq\left\{A B B^{\dagger} A^{(1,2,3)}\right\}$. & $\langle 54\rangle$ & $\left\{A B(A B)^{(1)}\right\} \supseteq\left\{A B B^{\dagger} A^{(1,2,4)}\right\}$. \\
\hline$\langle 55\rangle$ & $\left\{A B(A B)^{(1)}\right\} \supseteq\left\{A B B^{\dagger} A^{(1,3,4)}\right\}$. & $\langle 56\rangle$ & $\left\{A B(A B)^{(1)}\right\} \ni A B B^{\dagger} A^{\dagger}$ \\
\hline$\langle 57\rangle$ & $\left\{A B(A B)^{(1)}\right\} \supseteq\left\{A B B^{(1)} A^{(1,4)}\right\}$. & $\langle 58\rangle$ & $\left\{A B(A B)^{(1)}\right\} \supseteq\left\{A B B^{(1)} A^{(1,2,4)}\right\}$. \\
\hline$\langle 59\rangle$ & $\left\{A B(A B)^{(1)}\right\} \supseteq\left\{A B B^{(1)} A^{(1,3,4)}\right\}$. & $\langle 60\rangle$ & $\left\{A B(A B)^{(1)}\right\} \supseteq\left\{A B B^{(1)} A^{\dagger}\right\}$ \\
\hline$\langle 61\rangle$ & $\left\{(A B)^{(1)} A B\right\} \supseteq\left\{B^{(1)} A^{\dagger} A B\right\}$ & $\langle 62\rangle$ & $\left\{(A B)^{(1)} A B\right\} \supseteq\left\{B^{(1,2)} A^{\dagger} A B\right\}$. \\
\hline$\langle 63\rangle$ & $\left\{(A B)^{(1)} A B\right\} \supseteq\left\{B^{(1,3)} A^{\dagger} A B\right\}$ & $\langle 64\rangle$ & $\left\{(A B)^{(1)} A B\right\} \supseteq\left\{B^{(1,4)} A^{\dagger} A B\right\}$ \\
\hline$\langle 65\rangle$ & $\left\{(A B)^{(1)} A B\right\} \supseteq\left\{B^{(1,2,3)} A^{\dagger} A B\right\}$. & $\langle 66\rangle$ & $\left\{(A B)^{(1)} A B\right\} \supseteq\left\{B^{(1,2,4)} A^{\dagger} A B\right\}$. \\
\hline$\langle 67\rangle$ & $\left\{(A B)^{(1)} A B\right\} \supseteq\left\{B^{(1,3,4)} A^{\dagger} A B\right\}$. & $\langle 68\rangle$ & $\left\{(A B)^{(1)} A B\right\} \ni B^{\dagger} A^{\dagger} A B$ \\
\hline$\langle 69\rangle$ & $\left\{(A B)^{(1)} A B\right\} \supseteq\left\{B^{(1,3)} A^{(1)} A B\right\}$ & $\langle 70\rangle$ & $\left\{(A B)^{(1)} A B\right\} \supseteq\left\{B^{(1,4)} A^{(1)} A B\right\}$ \\
\hline$\langle 71\rangle$ & $\left\{(A B)^{(1)} A B\right\} \supseteq\left\{B^{(1,2,3)} A^{(1)} A B\right\}$. & $\langle 72\rangle$ & $\left\{(A B)^{(1)} A B\right\} \supseteq\left\{B^{(1,3,4)} A^{(1)} A B\right\}$. \\
\hline$\langle 73\rangle$ & $\left\{B(A B)^{(1)} A\right\} \supseteq\left\{B B^{(1)} A^{(1)} A\right\}$ & $\langle 74\rangle$ & $\left\{B(A B)^{(1)} A\right\} \supseteq\left\{B B^{\dagger} A^{(1)} A\right\}$ \\
\hline$\langle 75\rangle$ & $\left\{B(A B)^{(1)} A\right\} \supseteq\left\{B B^{(1)} A^{\dagger} A\right\}$. & $\langle 76\rangle$ & $\left\{B(A B)^{(1)} A\right\} \ni B B^{\dagger} A^{\dagger} A$ \\
\hline
\end{tabular}

$\langle 77\rangle\left\{\left[\left(A^{\dagger}\right)^{*} B\right]^{(1)}\right\} \ni B^{\dagger} A^{*}$ and $/$ or $\left\{\left[A\left(B^{\dagger}\right)^{*}\right]^{(1)}\right\} \ni B^{*} A^{\dagger}$

$\langle 78\rangle\left\{\left(A^{*} A B\right)^{(1)}\right\} \ni B^{\dagger}\left(A^{*} A\right)^{\dagger}$ and/or $\left\{\left(A B B^{*}\right)^{(1)}\right\} \ni\left(B B^{*}\right)^{\dagger} A^{\dagger}$.

$\langle 79\rangle\left\{\left(A^{*} A B B^{*}\right)^{(1)}\right\} \ni\left(B B^{*}\right)^{\dagger}\left(A^{*} A\right)^{\dagger}$ and/or $\left\{\left(B B^{*} A^{*} A\right)^{(1)}\right\} \ni\left(A^{*} A\right)^{\dagger}\left(B B^{*}\right)^{\dagger}$.

$\langle 80\rangle\left\{\left[\left(A^{*} A\right)^{1 / 2}\left(B B^{*}\right)^{1 / 2}\right]^{(1)}\right\} \quad \ni \quad\left[\left(B B^{*}\right)^{1 / 2}\right]^{\dagger}\left[\left(A^{*} A\right)^{1 / 2}\right]^{\dagger} \quad$ and $/$ or $\quad\left\{\left[\left(B B^{*}\right)^{1 / 2}\left(A^{*} A\right)^{1 / 2}\right]^{(1)}\right\} \quad \ni$ $\left.\left[\left(A^{*} A\right)^{1 / 2}\right]^{\dagger}\left(B B^{*}\right)^{1 / 2}\right]^{\dagger}$.

$\langle 81\rangle\left\{\left(A A^{*} A B B^{*} B\right)^{(1)}\right\} \ni\left(B B^{*} B\right)^{\dagger}\left(A A^{*} A\right)^{\dagger}$.

$\langle 82\rangle\left\{\left(A^{\dagger} A B\right)^{(1)}\right\} \ni B^{\dagger} A^{\dagger} A$ and/or $\left\{\left(A B B^{\dagger}\right)^{(1)}\right\} \ni B B^{\dagger} A^{\dagger}$.

$\langle 83\rangle\left\{\left(A^{\dagger} A B B^{\dagger}\right)^{(1)}\right\} \ni B B^{\dagger} A^{\dagger} A$ and $/$ or $\left\{\left(B B^{\dagger} A^{\dagger} A\right)^{(1)}\right\} \ni A^{\dagger} A B B^{\dagger}$.

$\langle 84\rangle\left\{\left(F_{A} B B^{\dagger}\right)^{(1)}\right\} \ni B B^{\dagger} F_{A}$ and $/$ or $\left\{\left(B B^{\dagger} F_{A}\right)^{(1)}\right\} \ni F_{A} B B^{\dagger}$.

$\langle 85\rangle\left\{\left(A^{\dagger} A E_{B}\right)^{(1)}\right\} \ni E_{B} A^{\dagger} A$ and $/$ or $\left\{\left(E_{B} A^{\dagger} A\right)^{(1)}\right\} \ni A^{\dagger} A E_{B}$.

$\langle 86\rangle\left\{\left(F_{A} E_{B}\right)^{(1)}\right\} \in E_{B} F_{A}$ and/or $\left\{\left(E_{B} F_{A}\right)^{(1)}\right\} \ni F_{A} E_{B}$. 
$\langle 87\rangle A B B^{\dagger} A^{\dagger} A B=A B$ and/or $A E_{B} F_{A} B=0$.

$\langle 88\rangle B^{\dagger} A^{\dagger} A B B^{\dagger} A^{\dagger}=B^{\dagger} A^{\dagger}$ and/or $B^{\dagger} F_{A} E_{B} A^{\dagger}=0$.

$\langle 89\rangle A B B^{\dagger} F_{A}=0$ and/or $E_{B} A^{\dagger} A B=0$.

$\langle 90\rangle A E_{B} F_{A}=0$ and/or $E_{B} F_{A} B=0$.

$\langle 91\rangle A^{\dagger} A B B^{\dagger}=B B^{\dagger} A^{\dagger} A$ and/or $F_{A} B B^{\dagger}=B B^{\dagger} F_{A}, A^{\dagger} A E_{B}=E_{B} A^{\dagger} A, F_{A} E_{B}=E_{B} F_{A}$.

$\langle 92\rangle\left(A^{\dagger} A B B^{\dagger}\right)^{2}=A^{\dagger} A B B^{\dagger}$ and $/$ or $\left(B B^{\dagger} A^{\dagger} A\right)^{2}=B B^{\dagger} A^{\dagger} A$.

$\langle 93\rangle\left(A B B^{\dagger} A^{\dagger}\right)^{2}=A B B^{\dagger} A^{\dagger}$ and $/$ or $\left(B^{\dagger} A^{\dagger} A B\right)^{2}=B^{\dagger} A^{\dagger} A B$.

$\langle 94\rangle\left(F_{A} B B^{\dagger}\right)^{2}=F_{A} B B^{\dagger}$ and $/$ or $\left(B B^{\dagger} F_{A}\right)^{2}=B B^{\dagger} F_{A}$.

$\langle 95\rangle\left(A^{\dagger} A E_{B}\right)^{2}=A^{\dagger} A E_{B}$ and/or $\left(E_{B} A^{\dagger} A\right)^{2}=E_{B} A^{\dagger} A$.

$\langle 96\rangle\left(F_{A} E_{B}\right)^{2}=F_{A} E_{B}$ and/or $\left(E_{B} F_{A}\right)^{2}=E_{B} F_{A}$.

$\langle 97\rangle\left(A E_{B} A^{\dagger}\right)^{2}=A E_{B} A^{\dagger}$ and $/$ or $\left(B^{\dagger} F_{A} B\right)^{2}=B^{\dagger} F_{A} B$.

$\langle 98\rangle\left(A^{\dagger} A B B^{\dagger}\right)^{\dagger}=B B^{\dagger} A^{\dagger} A$ and/or $\left(B B^{\dagger} A^{\dagger} A\right)^{\dagger}=A^{\dagger} A B B^{\dagger}$.

$\langle 99\rangle\left(B B^{\dagger} F_{A}\right)^{\dagger}=F_{A} B B^{\dagger}$ and $/$ or $\left(F_{A} B B^{\dagger}\right)^{\dagger}=B B^{\dagger} F_{A}$.

$\langle 100\rangle\left(A^{\dagger} A E_{B}\right)^{\dagger}=E_{B} A^{\dagger} A$ and $/$ or $\left(E_{B} A^{\dagger} A\right)^{\dagger}=A^{\dagger} A E_{B}$.

$\langle 101\rangle\left(F_{A} E_{B}\right)^{\dagger}=E_{B} F_{A}$ and/or $\left(E_{B} F_{A}\right)^{\dagger}=F_{A} E_{B}$.

$\langle 102\rangle\left(A^{\dagger} A-B B^{\dagger}\right)^{\dagger}=A^{\dagger} A-B B^{\dagger}$ and/or $\left(B B^{\dagger}-A^{\dagger} A\right)^{\dagger}=B B^{\dagger}-A^{\dagger} A$.

$\langle 103\rangle\left(I_{n}-A^{\dagger} A-B B^{\dagger}\right)^{\dagger}=I_{n}-A^{\dagger} A-B B^{\dagger}$.

$\langle 104\rangle\left(I_{m}-A B B^{\dagger} A^{\dagger}\right)^{\dagger}=I_{m}-A B B^{\dagger} A^{\dagger}$ and/or $\left(I_{p}-B^{\dagger} A^{\dagger} A B\right)^{\dagger}=I_{p}-B^{\dagger} A^{\dagger} A B$.

$\langle 105\rangle\left(I_{n}-A^{\dagger} A B B^{\dagger}\right)^{\dagger}=I_{n}-A^{\dagger} A B B^{\dagger}$ and/or $\left(I_{n}-B B^{\dagger} A^{\dagger} A\right)^{\dagger}=I_{n}-B B^{\dagger} A^{\dagger} A$.

$\langle 106\rangle\left(I_{m}-A E_{B} A^{\dagger}\right)^{\dagger}=I_{m}-A E_{B} A^{\dagger}$ and/or $\left(I_{p}-B^{\dagger} F_{A} B\right)^{\dagger}=I_{p}-B^{\dagger} F_{A} B$.

$\langle 107\rangle\left(I_{n}-F_{A} B B^{\dagger}\right)^{\dagger}=I_{n}-F_{A} B B^{\dagger}$ and/or $\left(I_{n}-B B^{\dagger} F_{A}\right)^{\dagger}=I_{n}-B B^{\dagger} F_{A}$.

$\langle 108\rangle\left(I_{n}-A^{\dagger} A E_{B}\right)^{\dagger}=I_{n}-A^{\dagger} A E_{B}$ and/or $\left(I_{n}-E_{B} A^{\dagger} A\right)^{\dagger}=I_{n}-E_{B} A^{\dagger} A$.

$\langle 109\rangle\left(I_{n}-F_{A} E_{B}\right)^{\dagger}=I_{n}-F_{A} E_{B}$ and/or $\left(I_{n}-E_{B} F_{A}\right)^{\dagger}=I_{n}-E_{B} F_{A}$.

$\langle 110\rangle\left[A^{*}, B\right]\left[A^{*}, B\right]^{\dagger}=A^{\dagger} A+B B^{\dagger}-A^{\dagger} A B B^{\dagger}$ and $/$ or $\left[A^{*}, B\right]\left[A^{*}, B\right]^{\dagger}=A^{\dagger} A+B B^{\dagger}-B B^{\dagger} A^{\dagger} A$.

$\langle 111\rangle\left[F_{A}, B\right]\left[F_{A}, B\right]^{\dagger}=F_{A}+B B^{\dagger}-F_{A} B B^{\dagger}$ and $/$ or $\left[F_{A}, B\right]\left[F_{A}, B\right]^{\dagger}=F_{A}+B B^{\dagger}-B B^{\dagger} F_{A}$.

$\langle 112\rangle\left[A^{*}, E_{B}\right]\left[A^{*}, E_{B}\right]^{\dagger}=A^{\dagger} A+E_{B}-A^{\dagger} A E_{B}$ and $/$ or $\left[A^{*}, E_{B}\right]\left[A^{*}, E_{B}\right]^{\dagger}=A^{\dagger} A+E_{B}-E_{B} A^{\dagger} A$.

$\langle 113\rangle\left[F_{A}, E_{B}\right]\left[F_{A}, E_{B}\right]^{\dagger}=F_{A}+E_{B}-F_{A} E_{B}$ and $/$ or $\left[F_{A}, E_{B}\right]\left[F_{A}, E_{B}\right]^{\dagger}=F_{A}+E_{B}-E_{B} F_{A}$.

$\langle 114\rangle A B B^{(1,3)} A^{(1)} A B$ is invariant with respect to the choice of $A^{(1)}$ and $B^{(1,3)}$, i.e., $A B B^{(1,3)} A^{(1)} A B=$ $A B B^{\dagger} A^{\dagger} A B$ holds for all $A^{(1)}$ and $B^{(1,3)}$.

$\langle 115\rangle A B B^{(1)} A^{(1,4)} A B$ is invariant with respect to the choice of $A^{(1,4)}$ and $B^{(1)}$, i.e., $A B B^{(1)} A^{(1,4)} A B=$ $A B B^{\dagger} A^{\dagger} A B$ holds for all $A^{(1,4)}$ and $B^{(1)}$.

$\langle 116\rangle r\left[A^{*}, B\right]=r(A)+r(B)-r(A B)$.

$\langle 117\rangle r\left[F_{A}, B\right]=r\left(F_{A}\right)+r(B)-r\left(F_{A} B\right)$.

$\langle 118\rangle r\left[A, E_{B}\right]=r(A)+r\left(E_{B}\right)-r\left(A E_{B}\right)$.

$\langle 119\rangle r\left[F_{A}, E_{B}\right]=r\left(F_{A}\right)+r\left(E_{B}\right)-r\left(F_{A} E_{B}\right)$.

$\langle 120\rangle r\left(I_{n}-A^{\dagger} A B B^{\dagger}\right)=n-r\left(A^{\dagger} A B B^{\dagger}\right)$ and/or $r\left(I_{n}-B B^{\dagger} A^{\dagger} A\right)=n-r\left(B B^{\dagger} A^{\dagger} A\right)$.

$\langle 121\rangle r\left(I_{m}-A B B^{\dagger} A^{\dagger}\right)=m-r\left(A B B^{\dagger} A^{\dagger}\right)$ and/or $r\left(I_{p}-B^{\dagger} A^{\dagger} A B\right)=p-r\left(B^{\dagger} A^{\dagger} A B\right)$. 
$\langle 122\rangle r\left(I_{n}-F_{A} B B^{\dagger}\right)=n-r\left(F_{A} B B^{\dagger}\right)$ and/or $r\left(I_{n}-B B^{\dagger} F_{A}\right)=n-r\left(B B^{\dagger} F_{A}\right)$.

$\langle 123\rangle r\left(I_{n}-A^{\dagger} A E_{B}\right)=n-r\left(A^{\dagger} A E_{B}\right)$ and/or $r\left(I_{n}-E_{B} A^{\dagger} A\right)=n-r\left(E_{B} A^{\dagger} A\right)$.

$\langle 124\rangle r\left(I_{n}-F_{A} E_{B}\right)=n-r\left(F_{A} E_{B}\right)$ and/or $r\left(I_{n}-E_{B} F_{A}\right)=n-r\left(E_{B} F_{A}\right)$.

$\langle 125\rangle r\left(I_{m}-A E_{B} A^{\dagger}\right)=m-r\left(A E_{B} A^{\dagger}\right)$ and/or $r\left(I_{p}-B^{\dagger} F_{A} B\right)=p-r\left(B^{\dagger} F_{A} B\right)$.

$\langle 126\rangle r\left(A^{\dagger} A-A^{\dagger} A B B^{\dagger}\right)=r\left(A^{\dagger} A\right)-r\left(A^{\dagger} A B B^{\dagger}\right)$ and/or $r\left(B B^{\dagger}-A^{\dagger} A B B^{\dagger}\right)=r\left(B B^{\dagger}\right)-r\left(A^{\dagger} A B B^{\dagger}\right)$.

$\langle 127\rangle r\left(A^{\dagger} A-A^{\dagger} A E_{B}\right)=r\left(A^{\dagger} A\right)-r\left(A^{\dagger} A E_{B}\right)$ and/or $r\left(B B^{\dagger}-F_{A} B B^{\dagger}\right)=r\left(B B^{\dagger}\right)-r\left(F_{A} B B^{\dagger}\right)$.

$\langle 128\rangle r\left(F_{A}-F_{A} B B^{\dagger}\right)=r\left(F_{A}\right)-r\left(F_{A} B B^{\dagger}\right)$ and/or $r\left(E_{B}-A^{\dagger} A E_{B}\right)=r\left(E_{B}\right)-r\left(A^{\dagger} A E_{B}\right)$.

$\langle 129\rangle r\left(F_{A}-F_{A} E_{B}\right)=r\left(F_{A}\right)-r\left(F_{A} E_{B}\right)$ and $r\left(E_{B}-F_{A} E_{B}\right)=r\left(E_{B}\right)-r\left(F_{A} E_{B}\right)$.

$\langle 130\rangle \mathscr{R}\left(I_{n}-A^{\dagger} A B B^{\dagger}\right) \cap \mathscr{R}\left(A^{\dagger} A B B^{\dagger}\right)=\{0\}$ and/or $\mathscr{R}\left(I_{n}-B B^{\dagger} A^{\dagger} A\right) \cap \mathscr{R}\left(B B^{\dagger} A^{\dagger} A\right)=\{0\}$.

$\langle 131\rangle \mathscr{R}\left(I_{n}-F_{A} B B^{\dagger}\right) \cap \mathscr{R}\left(F_{A} B B^{\dagger}\right)=\{0\}$ and $/$ or $\mathscr{R}\left(I_{n}-B B^{\dagger} F_{A}\right) \cap \mathscr{R}\left(B B^{\dagger} F_{A}\right)=\{0\}$.

$\langle 132\rangle \mathscr{R}\left(I_{n}-A^{\dagger} A E_{B}\right) \cap \mathscr{R}\left(A^{\dagger} A E_{B}\right)=\{0\}$ and/or $\mathscr{R}\left(I_{n}-E_{B} A^{\dagger} A\right) \cap \mathscr{R}\left(E_{B} A^{\dagger} A\right)=\{0\}$.

$\langle 133\rangle \mathscr{R}\left(I_{n}-F_{A} E_{B}\right) \cap \mathscr{R}\left(F_{A} E_{B}\right)=\{0\}$ and/or $\mathscr{R}\left(I_{n}-E_{B} F_{A}\right) \cap \mathscr{R}\left(E_{B} F_{A}\right)=\{0\}$.

$\langle 134\rangle \mathscr{R}\left(I_{m}-A B B^{\dagger} A^{\dagger}\right) \cap \mathscr{R}\left(A B B^{\dagger} A^{\dagger}\right)=\{0\}$ and $/$ or $\mathscr{R}\left[\left(I_{m}-A B B^{\dagger} A^{\dagger}\right)^{*}\right] \cap \mathscr{R}\left[\left(A B B^{\dagger} A^{\dagger}\right)^{*}\right]=\{0\}$.

$\langle 135\rangle \mathscr{R}\left(I_{p}-B^{\dagger} A^{\dagger} A B\right) \cap \mathscr{R}\left(B^{\dagger} A^{\dagger} A B\right)=\{0\}$ and/or $\mathscr{R}\left[\left(I_{p}-B^{\dagger} A^{\dagger} A B\right)^{*}\right] \cap \mathscr{R}\left[\left(B^{\dagger} A^{\dagger} A B\right)^{*}\right]=\{0\}$.

$\langle 136\rangle \mathscr{R}\left(I_{m}-A E_{B} A^{\dagger}\right) \cap \mathscr{R}\left(A E_{B} A^{\dagger}\right)=\{0\}$ and/or $\mathscr{R}\left[\left(I_{m}-A E_{B} A^{\dagger}\right)^{*}\right] \cap \mathscr{R}\left[\left(A E_{B} A^{\dagger}\right)^{*}\right]=\{0\}$.

$\langle 137\rangle \mathscr{R}\left(I_{p}-B^{\dagger} F_{A} B\right) \cap \mathscr{R}\left(B^{\dagger} F_{A} B\right)=\{0\}$ and $/$ or $\mathscr{R}\left[\left(I_{p}-B^{\dagger} F_{A} B\right)^{*}\right] \cap \mathscr{R}\left[\left(B^{\dagger} F_{A} B\right)^{*}\right]=\{0\}$.

$\langle 138\rangle \mathbb{C}^{n}=\mathscr{R}\left(I_{n}-A^{\dagger} A B B^{\dagger}\right) \oplus \mathscr{R}\left(A^{\dagger} A B B^{\dagger}\right)$ and $/$ or $\mathbb{C}^{n}=\mathscr{R}\left(I_{n}-B B^{\dagger} A^{\dagger} A\right) \oplus \mathscr{R}\left(B B^{\dagger} A^{\dagger} A\right)$.

$\langle 139\rangle \mathbb{C}^{n}=\mathscr{R}\left(I_{n}-F_{A} B B^{\dagger}\right) \oplus \mathscr{R}\left(F_{A} B B^{\dagger}\right)$ and $/$ or $\mathbb{C}^{n}=\mathscr{R}\left(I_{n}-B B^{\dagger} F_{A}\right) \oplus \mathscr{R}\left(B B^{\dagger} F_{A}\right)$.

$\langle 140\rangle \mathbb{C}^{n}=\mathscr{R}\left(I_{n}-A^{\dagger} A E_{B}\right) \oplus \mathscr{R}\left(A^{\dagger} A E_{B}\right)$ and $/$ or $\mathbb{C}^{n}=\mathscr{R}\left(I_{n}-E_{B} A^{\dagger} A\right) \oplus \mathscr{R}\left(E_{B} A^{\dagger} A\right)$.

$\langle 141\rangle \mathbb{C}^{n}=\mathscr{R}\left(I_{n}-F_{A} E_{B}\right) \oplus \mathscr{R}\left(F_{A} E_{B}\right)$ and/or $\mathbb{C}^{n}=\mathscr{R}\left(I_{n}-E_{B} F_{A}\right) \oplus \mathscr{R}\left(E_{B} F_{A}\right)$.

$\langle 142\rangle \mathbb{C}^{m}=\mathscr{R}\left(I_{m}-A B B^{\dagger} A^{\dagger}\right) \oplus \mathscr{R}\left(A B B^{\dagger} A^{\dagger}\right)$ and $/$ or $\mathbb{C}^{m}=\mathscr{R}\left[\left(I_{m}-A B B^{\dagger} A^{\dagger}\right)^{*}\right] \oplus \mathscr{R}\left[\left(A B B^{\dagger} A^{\dagger}\right)^{*}\right]$.

$\langle 143\rangle \mathbb{C}^{p}=\mathscr{R}\left(I_{p}-B^{\dagger} A^{\dagger} A B\right) \oplus \mathscr{R}\left(B^{\dagger} A^{\dagger} A B\right)$ and/or $\mathbb{C}^{p}=\mathscr{R}\left[\left(I_{p}-B^{\dagger} A^{\dagger} A B\right)^{*}\right] \oplus \mathscr{R}\left[\left(B^{\dagger} A^{\dagger} A B\right)^{*}\right]=\{0\}$.

$\langle 144\rangle \mathbb{C}^{m}=\mathscr{R}\left(I_{m}-A E_{B} A^{\dagger}\right) \oplus \mathscr{R}\left(A E_{B} A^{\dagger}\right)$ and/or $\mathbb{C}^{m}=\mathscr{R}\left[\left(I_{m}-A E_{B} A^{\dagger}\right)^{*}\right] \oplus \mathscr{R}\left[\left(A E_{B} A^{\dagger}\right)^{*}\right]$

$\langle 145\rangle \mathbb{C}^{p}=\mathscr{R}\left(I_{p}-B^{\dagger} F_{A} B\right) \oplus \mathscr{R}\left(B^{\dagger} F_{A} B\right)$ and $/$ or $\mathbb{C}^{p}=\mathscr{R}\left[\left(I_{p}-B^{\dagger} F_{A} B\right)^{*}\right] \oplus \mathscr{R}\left[\left(B^{\dagger} F_{A} B\right)^{*}\right]$.

$\langle 146\rangle \operatorname{dim}\left[\mathscr{R}\left(A^{*}\right) \cap \mathscr{R}(B)\right]=r(A B)$ and $/$ or $\operatorname{dim}\left[\mathscr{R}\left(F_{A}\right) \cap \mathscr{R}(B)\right]=r\left(F_{A} B\right), \operatorname{dim}\left[\mathscr{R}\left(A^{*}\right) \cap \mathscr{R}\left(E_{B}\right)\right]=r\left(A E_{B}\right)$, $\operatorname{dim}\left[\mathscr{R}\left(F_{A}\right) \cap \mathscr{R}\left(E_{B}\right)\right]=r\left(F_{A} E_{B}\right)$.

$\langle 147\rangle \mathscr{R}\left(B B^{\dagger} A^{\dagger} A\right) \subseteq \mathscr{R}\left(A^{\dagger} A\right)$ and/or $\mathscr{R}\left(A^{\dagger} A B B^{\dagger}\right) \subseteq \mathscr{R}\left(B B^{\dagger}\right)$.

$\langle 148\rangle \mathscr{R}\left(B B^{\dagger} F_{A}\right) \subseteq \mathscr{R}\left(F_{A}\right)$ and/or $\mathscr{R}\left(F_{A} B B^{\dagger}\right) \subseteq \mathscr{R}\left(B B^{\dagger}\right)$.

$\langle 149\rangle \mathscr{R}\left(E_{B} A^{\dagger} A\right) \subseteq \mathscr{R}\left(A^{\dagger} A\right)$ and/or $\mathscr{R}\left(A^{\dagger} A E_{B}\right) \subseteq \mathscr{R}\left(E_{B}\right)$.

$\langle 150\rangle \mathscr{R}\left(E_{B} F_{A}\right) \subseteq \mathscr{R}\left(F_{A}\right)$ and/or $\mathscr{R}\left(F_{A} E_{B}\right) \subseteq \mathscr{R}\left(E_{B}\right)$.

$\langle 151\rangle \mathscr{R}\left(A^{\dagger} A B B^{\dagger}\right)=\mathscr{R}\left(A^{\dagger} A\right) \cap \mathscr{R}\left(B B^{\dagger}\right)$ and/or $\mathscr{R}\left(B B^{\dagger} A^{\dagger} A\right)=\mathscr{R}\left(A^{\dagger} A\right) \cap \mathscr{R}\left(B B^{\dagger}\right)$.

$\langle 152\rangle \mathscr{R}\left(F_{A} B B^{\dagger}\right)=\mathscr{R}\left(F_{A}\right) \cap \mathscr{R}\left(B B^{\dagger}\right)$ and/or $\mathscr{R}\left(B B^{\dagger} F_{A}\right)=\mathscr{R}\left(F_{A}\right) \cap \mathscr{R}\left(B B^{\dagger}\right)$.

$\langle 153\rangle \mathscr{R}\left(A^{\dagger} A E_{B}\right)=\mathscr{R}\left(A^{\dagger} A\right) \cap \mathscr{R}\left(E_{B}\right)$ and $/$ or $\mathscr{R}\left(E_{B} A^{\dagger} A\right)=\mathscr{R}\left(A^{\dagger} A\right) \cap \mathscr{R}\left(E_{B}\right)$.

$\langle 154\rangle \mathscr{R}\left(F_{A} E_{B}\right)=\mathscr{R}\left(F_{A}\right) \cap \mathscr{R}\left(E_{B}\right)$ and/or $\mathscr{R}\left(E_{B} F_{A}\right)=\mathscr{R}\left(F_{A}\right) \cap \mathscr{R}\left(E_{B}\right)$.

$\langle 155\rangle \mathscr{R}\left(A^{\dagger} A B B^{\dagger}\right)=\mathscr{R}\left(B B^{\dagger} A^{\dagger} A\right)$ and/or $\mathscr{R}\left(A^{\dagger} A E_{B}\right)=\mathscr{R}\left(E_{B} A^{\dagger} A\right), \mathscr{R}\left(F_{A} B B^{\dagger}\right)=\mathscr{R}\left(B B^{\dagger} F_{A}\right), \mathscr{R}\left(F_{A} E_{B}\right)=$ $\mathscr{R}\left(E_{B} F_{A}\right)$.

$\langle 156\rangle \mathscr{R}(A B) \cap \mathscr{R}\left(A E_{B}\right)=\{0\}$ and/or $\mathscr{R}\left(F_{A} B\right) \cap \mathscr{R}\left(F_{A} E_{B}\right)=\{0\}, \mathscr{R}\left(B^{*} A^{*}\right) \cap \mathscr{R}\left(B^{*} F_{A}\right)=\{0\}, \mathscr{R}\left(E_{B} A^{*}\right) \cap$ $\mathscr{R}\left(E_{B} F_{A}\right)=\{0\}$. 
$\langle 157\rangle \mathscr{R}(A)=\mathscr{R}(A B) \oplus \mathscr{R}\left(A E_{B}\right)$ and/or $\mathscr{N}(A)=\mathscr{R}\left(F_{A} B\right) \oplus \mathscr{R}\left(F_{A} E_{B}\right), \mathscr{R}\left(B^{*}\right)=\mathscr{R}\left(B^{*} A^{*}\right) \oplus \mathscr{R}\left(B^{*} F_{A}\right), \mathscr{N}\left(B^{*}\right)=$ $\mathscr{R}\left(E_{B} A^{*}\right) \oplus \mathscr{R}\left(E_{B} F_{A}\right)$.

$\langle 158\rangle \mathscr{R}\left(A^{\dagger} A-B B^{\dagger}\right) \cap \mathscr{R}\left(A^{\dagger} A+B B^{\dagger}-I_{n}\right)=\{0\}$.

$\langle 159\rangle\left[\mathscr{R}\left(A^{*}\right)+\mathscr{R}(B)\right] \cap\left[\mathscr{R}\left(A^{*}\right)+\mathscr{R}(B)^{\perp}\right] \cap\left[\mathscr{R}\left(A^{*}\right)^{\perp}+\mathscr{R}(B)\right] \cap\left[\mathscr{R}\left(A^{*}\right)^{\perp}+\mathscr{R}(B)^{\perp}\right]=\{0\}$.

$\langle 160\rangle\left[\mathscr{R}\left(A^{*}\right) \cap \mathscr{R}(B)\right] \oplus\left[\mathscr{R}\left(A^{*}\right) \cap \mathscr{R}(B)^{\perp}\right] \oplus\left[\mathscr{R}\left(A^{*}\right)^{\perp} \cap \mathscr{R}(B)\right] \oplus\left[\mathscr{R}\left(A^{*}\right)^{\perp} \cap \mathscr{R}(B)^{\perp}\right]=\mathbb{C}^{n}$.

$\langle 161\rangle P_{\mathscr{R}\left(A^{*}\right) \cap \mathscr{R}(B)}=A^{\dagger} A B B^{\dagger}$ and/or $P_{\mathscr{R}(B) \cap \mathscr{R}\left(A^{*}\right)}=B B^{\dagger} A^{\dagger} A$.

$\langle 162\rangle$ The matrix equations $A^{*} X=B B^{\dagger} A^{*}$ and/or $B Y=A^{\dagger} A B$ are solvable.

$\langle 163\rangle$ The matrix equations $F_{A} X=B B^{\dagger} F_{A}$ and/or $B Y=F_{A} B$ are solvable.

$\langle 164\rangle$ The matrix equations $A^{*} X=E_{B} A^{*}$ and/or $E_{B} Y=A^{\dagger} A E_{B}$ are solvable.

$\langle 165\rangle$ The matrix equations $F_{A} X=E_{B} F_{A}$ and/or $E_{B} Y=F_{A} E_{B}$ are solvable.

Proof. Setting all sides of $(8.25)-(8.27)$ equal to zero lead to the equivalence of $\langle 1\rangle-\langle 48\rangle,\langle 87\rangle$, and $\langle 116\rangle$. Setting all sides of (8.28) and (8.29) equal to zero leads to the equivalence of $\langle 114\rangle,\langle 115\rangle$, and $\langle 116\rangle$.

By (4.2),

$$
\begin{aligned}
& r\left(F_{A}\right)=n-r(A), \quad r\left(F_{A} B\right)=r\left[A^{*}, B\right]-r\left(A^{*}\right), \\
& r\left(E_{B}\right)=n-r(B), \quad r\left(A E_{B}\right)=r\left[A^{*}, B\right]-r(B), \\
& r\left[F_{A}, B\right]=r\left(F_{A}\right)+r\left(A^{\dagger} A B\right)=n-r(A)+r(A B), \\
& r\left[A^{*}, E_{B}\right]=r\left(A B B^{\dagger}\right)+r\left(E_{B}\right)=n-r(B)+r(A B), \\
& r\left[F_{A}, E_{B}\right]=r\left(F_{A}\right)+r\left(A^{\dagger} A E_{B}\right)=n-r(A)-r(B)-r\left[A^{*}, B\right], \\
& r\left(F_{A} E_{B}\right)=r\left[A^{*}, E_{B}\right]-r\left(A^{*}\right)=n-r(A)-r(B)+r(A B) .
\end{aligned}
$$

Substituting (9.2)-9.7) into the three rank equalities in $\langle 117\rangle-\langle 119\rangle$ and simplifying, we obtain the equivalence of $\langle 116\rangle-\langle 119\rangle$.

Applying 4.10 to $I_{n}-A^{\dagger} A B B^{\dagger}, I_{m}-A B B^{\dagger} A^{\dagger}$, and $I_{p}-B^{\dagger} A^{\dagger} A B$, and simplifying by (4.2) and (4.3), we obtain

$$
\begin{aligned}
& r\left(I_{n}-A^{\dagger} A B B^{\dagger}\right)=r\left(I_{n}-B B^{\dagger} A^{\dagger} A\right)=r\left[\begin{array}{cc}
A A^{*} & A B B^{\dagger} \\
A^{*} & I_{n}
\end{array}\right]-r(A) \\
& =r\left[\begin{array}{cc}
A A^{*}-A B B^{\dagger} A^{*} & 0 \\
0 & I_{n}
\end{array}\right]-r(A)=n+r\left(A A^{*}-A B B^{\dagger} A^{*}\right)-r(A) \\
& =n+r\left[\begin{array}{cc}
B^{*} B & B^{*} A^{*} \\
A B & A A^{*}
\end{array}\right]-r(A)-r(B)=n+r\left(\left[A, B^{*}\right]^{*}\left[A, B^{*}\right]\right)-r(A)-r(B) \\
& =n+r(N)-r(A)-r(B) \text {, } \\
& r\left(I_{m}-A B B^{\dagger} A^{\dagger}\right)=r\left[\begin{array}{cc}
B^{*} B & B^{*} A^{\dagger} \\
A B & I_{m}
\end{array}\right]-r(B)=r\left[\begin{array}{cc}
B^{*} B-B^{*} A^{\dagger} A B & 0 \\
0 & I_{m}
\end{array}\right]-r(B) \\
& =r\left(B^{*} F_{A} B\right)-r(B)+m \\
& =r\left(F_{A} B\right)-r(B)+m=r(N)-r(A)-r(B)+m \text {, } \\
& r\left(I_{p}-B^{\dagger} A^{\dagger} A B\right)=r\left[\begin{array}{cc}
A A^{*} & A B \\
B^{\dagger} A^{*} & I_{p}
\end{array}\right]-r(A)=r\left[\begin{array}{cc}
A A^{*}-A B B^{\dagger} A^{*} & 0 \\
0 & I_{p}
\end{array}\right]-r(A) \\
& =r\left(A E_{B} A^{*}\right)-r(A)+p=r\left(E_{B} A^{*}\right)-r(A)+p \\
& =r(N)-r(A)-r(B)+p \text {. }
\end{aligned}
$$

Combining (9.8- -9.10 with $8.9-8.11$ yields

$$
\begin{aligned}
r\left(I_{n}-A^{\dagger} A B B^{\dagger}\right)-n+r\left(A^{\dagger} A B B^{\dagger}\right) & =r\left(I_{n}-B B^{\dagger} A^{\dagger} A\right)-n+r\left(B B^{\dagger} A^{\dagger} A\right) \\
& =r(N)-r(A)-r(B)+r(A B), \\
r\left(I_{m}-A B B^{\dagger} A^{\dagger}\right)-m+r\left(A B B^{\dagger} A^{\dagger}\right) & =r(N)-r(A)-r(B)+r(A B), \\
r\left(I_{p}-B^{\dagger} A^{\dagger} A B\right)-p+r\left(B^{\dagger} A^{\dagger} A B\right) & =r(N)-r(A)-r(B)+r(A B) .
\end{aligned}
$$


Setting all sides of 9.11 -9.13 equal to zero leads to the equivalence of $\langle 120\rangle,\langle 121\rangle$, and $\langle 116\rangle$. Replacing $A^{\dagger} A$ with $F_{A}$ and $B B^{\dagger}$ with $E_{B}$ respectively in $\langle 120\rangle$ and $\langle 121\rangle$ leads to the equivalence of $\langle 122\rangle-\langle 125\rangle$ with $\langle 116\rangle-\langle 118\rangle$.

Applying (8.25) to $B^{\dagger} F_{A} E_{B} A^{\dagger}=B^{\dagger} A^{\dagger}-B^{\dagger} A^{\dagger} A B B^{\dagger} A^{\dagger}$ and simplifying by Lemma 8.5, we obtain

$$
\begin{aligned}
r\left(B^{\dagger} F_{A} E_{B} A^{\dagger}\right) & =r\left(B^{\dagger} A^{\dagger}-B^{\dagger} A^{\dagger} A B B^{\dagger} A^{\dagger}\right) \\
& =r\left[\left(B^{\dagger}\right)^{*}, A^{\dagger}\right]+r\left(B^{\dagger} A^{\dagger}\right)-r\left(B^{\dagger}\right)-r\left(A^{\dagger}\right) \\
& =r(N)+r(A B)-r(B)-r(A) .
\end{aligned}
$$

Setting both sides of $(9.14)$ equal to zero leads to the equivalence of $\langle 88\rangle$ and $\langle 116\rangle$.

Replacing $A$ and $B$ in (9.14) with $\left(A^{\dagger}\right)^{*}, A^{*} A,\left(A^{*} A\right)^{1 / 2}, A A^{*} A, A^{\dagger} A,\left(B^{\dagger}\right)^{*}, B B^{*},\left(B B^{*}\right)^{1 / 2}, B B^{*} B$, and $B B^{\dagger}$, respectively, and simplifying by Lemma 8.5 , we also obtain the following rank formulas

$$
\begin{aligned}
& r\left[\left(A^{\dagger}\right)^{*} B-\left(A^{\dagger}\right)^{*} B B^{\dagger} A^{*}\left(A^{\dagger}\right)^{*} B\right] \\
& =r\left[A\left(B^{\dagger}\right)^{*}-A\left(B^{\dagger}\right)^{*} B^{*} A^{\dagger} A\left(B^{\dagger}\right)^{*}\right] \\
& =r\left[\left(A^{*} A\right) B-\left(A^{*} A\right) B B^{\dagger}\left(A^{*} A\right)^{\dagger}\left(A^{*} A\right) B\right] \\
& =r\left[A\left(B B^{*}\right)-A\left(B B^{*}\right)\left(B B^{*}\right)^{\dagger} A^{\dagger} A\left(B B^{*}\right)\right] \\
& =r\left[\left(A^{*} A\right)\left(B B^{*}\right)-\left(A^{*} A\right)\left(B B^{*}\right)\left(B B^{*}\right)^{\dagger}\left(A^{*} A\right)^{\dagger}\left(A^{*} A\right)\left(B B^{*}\right)\right] \\
& =r\left\{\left(A^{*} A\right)^{1 / 2}\left(B B^{*}\right)^{1 / 2}-\left(A^{*} A\right)^{1 / 2}\left(B B^{*}\right)^{1 / 2}\left[\left(B B^{*}\right)^{1 / 2}\right]^{\dagger}\left[\left(A^{*} A\right)^{1 / 2}\right]^{\dagger}\left(A^{*} A\right)^{1 / 2}\left(B B^{*}\right)^{1 / 2}\right\} \\
& =r\left[\left(A A^{*} A\right)\left(B B^{*} B\right)-\left(A A^{*} A\right)\left(B B^{*} B\right)\left(B B^{*} B\right)^{\dagger}\left(A A^{*} A\right)^{\dagger}\left(A A^{*} A\right)\left(B B^{*} B\right)\right] \\
& =r\left[A^{\dagger} A B-\left(A^{\dagger} A B\right) B^{\dagger} A^{\dagger} A\left(A^{\dagger} A B\right)\right] \\
& =r\left[A B B^{\dagger}-\left(A B B^{\dagger}\right) B B^{\dagger} A^{\dagger}\left(A B B^{\dagger}\right)\right] \\
& =r\left[A^{\dagger} A B B^{\dagger}-\left(A^{\dagger} A B B^{\dagger}\right) B B^{\dagger} A^{\dagger} A\left(A^{\dagger} A B B^{\dagger}\right)\right] \\
& =r(N)+r(A B)-r(A)-r(B) .
\end{aligned}
$$

Setting all sides of 9.15 equal to zero leads to the equivalence of $\langle 77\rangle-\langle 83\rangle$ and $\langle 116\rangle$.

Replacing $A$ and $B$ in (9.14) with $A A^{\dagger}, F_{A}, B B^{\dagger}$, and $E_{B}$, we further obtain

$$
\begin{aligned}
& r\left[F_{A} B B^{\dagger}-\left(F_{A} B B^{\dagger}\right) B B^{\dagger} F_{A}\left(F_{A} B B^{\dagger}\right)\right]=r\left[F_{A}, B\right]-r\left(F_{A}\right)-r(B)+r\left(F_{A} B\right), \\
& r\left[A^{\dagger} A E_{B}-\left(A^{\dagger} A E_{B}\right) E_{B} A^{\dagger} A\left(A^{\dagger} A E_{B}\right)\right]=r\left[A, E_{B}\right]-r(A)-r\left(E_{B}\right)+r\left(A E_{B}\right), \\
& r\left[F_{A} E_{B}-\left(F_{A} E_{B}\right) E_{B} F_{A}\left(F_{A} E_{B}\right)\right]=r\left[F_{A}, E_{B}\right]-r\left(F_{A}\right)-r\left(E_{B}\right)+r\left(F_{A} A E_{B}\right) .
\end{aligned}
$$

Setting all sides of $9.16-(9.18)$ equal to zero leads to the equivalence of $\langle 84\rangle-\langle 86\rangle$ and $\langle 117\rangle-\langle 119\rangle$.

Applying (4.2) to $A B B^{\dagger} F_{A}$ and $E_{B} A^{\dagger} A B$ and simplifying by Lemma 4.4 (c), we obtain

$$
\begin{aligned}
r\left(A B B^{\dagger} F_{A}\right) & =r\left[\begin{array}{c}
A B B^{\dagger} \\
A
\end{array}\right]-r(A)=r\left[\begin{array}{c}
A B B^{\dagger} \\
A-A B B^{\dagger}
\end{array}\right]-r(A) \\
& =r\left(A B B^{\dagger}\right)+r\left(A-A B B^{\dagger}\right)-r(A) \\
& =r(N)-r(A)-r(B)+r(A B), \\
r\left(E_{B} A^{\dagger} A B\right) & =r\left[B, A^{\dagger} A B\right]-r(B)=r\left[B-A^{\dagger} A B, A^{\dagger} A B\right]-r(B) \\
& =r\left(B-A^{\dagger} A B\right)+r\left(A^{\dagger} A B\right)-r(B) \\
& =r(N)-r(A)-r(B)+r(A B) .
\end{aligned}
$$

Setting all sides of 9.19 and 9.20 equal to zero leads to the equivalence of $\langle 89\rangle$ and $\langle 116\rangle$. Replacing both $A^{\dagger} A$ and $B B^{\dagger}$ with $F_{A}$ and $E_{B}$ in $\langle 89\rangle$ respectively leads to the equivalence of $\langle 90\rangle$ and $\langle 117\rangle$ and $\langle 118\rangle$.

Applying 4.24 to $A^{\dagger} A B B^{\dagger}-B B^{\dagger} A^{\dagger} A$ and simplifying, we obtain

$$
\begin{aligned}
& r\left(A^{\dagger} A B B^{\dagger}-B B^{\dagger} A^{\dagger} A\right)=r\left(F_{A} B B^{\dagger}-B B^{\dagger} F_{A}\right) \\
& =r\left(A^{\dagger} A E_{B}-E_{B} A^{\dagger} A\right)=r\left(F_{A} E_{B}-E_{B} F_{A}\right) \\
& =2 r\left[A^{\dagger} A, B B^{\dagger}\right]-2 r\left(A^{\dagger} A\right)-2 r\left(B B^{\dagger}\right)+2 r\left(A^{\dagger} A B B^{\dagger}\right) \\
& =2 r(N)-2 r(A)-2 r(B)+2 r(A B) .
\end{aligned}
$$

Setting both sides of 9.21 equal to zero leads to the equivalence of $\langle 91\rangle$ and $\langle 116\rangle$. 
Applying (4.18) to $\left(A^{\dagger} A B B^{\dagger}\right)^{2}-A^{\dagger} A B B^{\dagger},\left(B B^{\dagger} A^{\dagger} A\right)^{2}-B B^{\dagger} A^{\dagger} A,\left(A B B^{\dagger} A^{\dagger}\right)^{2}-A B B^{\dagger} A^{\dagger}$, and $\left(B^{\dagger} A^{\dagger} A B\right)^{2}-$ $B^{\dagger} A^{\dagger} A B$, and simplifying by (9.8)-(9.10) yield

$$
\begin{aligned}
r\left[\left(A^{\dagger} A B B^{\dagger}\right)^{2}-A^{\dagger} A B B^{\dagger}\right] & =r\left(I_{n}-A^{\dagger} A B B^{\dagger}\right)+r\left(A^{\dagger} A B B^{\dagger}\right)-n \\
& =r(N)-r(A)-r(B)+r(A B), \\
r\left[\left(B B^{\dagger} A^{\dagger} A\right)^{2}-B B^{\dagger} A^{\dagger} A\right] & =r\left(I_{n}-B B^{\dagger} A^{\dagger} A\right)+r\left(B B^{\dagger} A^{\dagger} A\right)-n \\
& =r(N)-r(A)-r(B)+r(A B), \\
r\left[\left(A B B^{\dagger} A^{\dagger}\right)^{2}-A B B^{\dagger} A^{\dagger}\right] & =r\left(I_{m}-A B B^{\dagger} A^{\dagger}\right)+r\left(A B B^{\dagger} A^{\dagger}\right)-m \\
& =r(N)-r(A)-r(B)+r(A B), \\
r\left[\left(B^{\dagger} A^{\dagger} A B\right)^{2}-B^{\dagger} A^{\dagger} A B\right] & =r\left(I_{p}-B^{\dagger} A^{\dagger} A B\right)+r\left(B^{\dagger} A^{\dagger} A B\right)-p \\
& =r(N)-r(A)-r(B)+r(A B) .
\end{aligned}
$$

Setting all sides of $9.22-9.25$ equal to zero leads to the equivalence of $\langle 92\rangle,\langle 93\rangle$, and $\langle 116\rangle$. Replacing $A^{\dagger} A$ with $F_{A}$ in $\langle 92\rangle$ leads to the equivalence of $\langle 94\rangle$ and $\langle 117\rangle$. Replacing $B B^{\dagger}$ with $E_{B}$ in $\langle 92\rangle$ leads to the equivalence of $\langle 95\rangle$ and $\langle 118\rangle$. Replacing both $A^{\dagger} A$ and and $B B^{\dagger}$ with $F_{A}$ and $E_{B}$ in $\langle 92\rangle$ leads to the equivalence of $\langle 96\rangle$ and $\langle 119\rangle$. Replacing $A^{\dagger} A$ and and $B B^{\dagger}$ with $F_{A}$ and $E_{B}$ in $\langle 93\rangle$ respectively leads to the equivalence of $\langle 97\rangle$ with $\langle 117\rangle$ and $\langle 118\rangle$.

Applying (4.13) to $\left(A^{\dagger} A B B^{\dagger}\right)^{\dagger}-B B^{\dagger} A^{\dagger} A$ and simplifying by (4.18) and (9.8), we obtain

$$
\begin{aligned}
& r\left[\left(A^{\dagger} A B B^{\dagger}\right)^{\dagger}-B B^{\dagger} A^{\dagger} A\right]=r\left[\left(A^{\dagger} A B B^{\dagger}\right)^{\dagger}-\left(A^{\dagger} A B B^{\dagger}\right)^{*}\right] \\
& =r\left[\left(A^{\dagger} A B B^{\dagger}\right)-\left(A^{\dagger} A B B^{\dagger}\right)\left(A^{\dagger} A B B^{\dagger}\right)^{*}\left(A^{\dagger} A B B^{\dagger}\right)\right] \\
& =r\left[\left(A^{\dagger} A B B^{\dagger}\right)-\left(A^{\dagger} A B B^{\dagger}\right)^{2}\right]=r\left(A^{\dagger} A B B^{\dagger}\right)+r\left(I_{n}-A^{\dagger} A B B^{\dagger}\right)-n \\
& =r(N)+r(A B)-r(A)-r(B) .
\end{aligned}
$$

Setting all sides of $(9.26)$ equal to zero leads to the equivalence of $\langle 98\rangle$ and $\langle 116\rangle$. Replacing $A^{\dagger} A$ with $F_{A}$ in $\langle 98\rangle$ leads to the equivalence of $\langle 99\rangle$ and $\langle 117\rangle$. Replacing $B B^{\dagger}$ with $E_{B}$ in $\langle 98\rangle$ leads to the equivalence of $\langle 100\rangle$ and $\langle 118\rangle$. Replacing both $A^{\dagger} A$ and $B B^{\dagger}$ with $F_{A}$ and $E_{B}$ in $\langle 98\rangle$ leads to the equivalence of $\langle 101\rangle$ and $\langle 119\rangle$.

Applying (4.13) to the difference $\left(A^{\dagger} A-B B^{\dagger}\right)^{\dagger}-\left(A^{\dagger} A-B B^{\dagger}\right)$ and simplifying by $4.19-4.22$ gives

$$
\begin{aligned}
& r\left[\left(A^{\dagger} A-B B^{\dagger}\right)^{\dagger}-\left(A^{\dagger} A-B B^{\dagger}\right)\right]=r\left[\left(A^{\dagger} A-B B^{\dagger}\right)-\left(A^{\dagger} A-B B^{\dagger}\right)^{3}\right] \\
& =r\left(A^{\dagger} A-B B^{\dagger}\right)+r\left(I_{n}-A^{\dagger} A+B B^{\dagger}\right)+r\left(I_{n}+A^{\dagger} A-B B^{\dagger}\right)-2 n \\
& =2 r\left[A^{\dagger} A, B B^{\dagger}\right]-r\left(A^{\dagger} A\right)-r\left(B B^{\dagger}\right)+r\left[I_{n}-A^{\dagger} A, B B^{\dagger}\right]+r\left[I_{n}-B B^{\dagger}, A^{\dagger} A\right]-2 n \\
& =2 r(N)-r(A)-r(B)+n-r(A)+r(A B)+n-r(B)+r(A B)-2 n \\
& =2 r(N)-2 r(A)-2 r(B)+2 r(A B) .
\end{aligned}
$$

Setting both sides of 9.27 equal to zero leads to the equivalence of $\langle 102\rangle$ and $\langle 116\rangle$. Replacing $A^{\dagger} A$ with $F_{A}$ in (9.27) yields

$$
r\left[\left(I_{n}-A^{\dagger} A-B B^{\dagger}\right)^{\dagger}-\left(I_{n}-A^{\dagger} A-B B^{\dagger}\right)\right]=r\left[F_{A}, B\right]-r\left(F_{A}\right)-r(B)+r\left(F_{A} B\right) .
$$

Setting both sides of 9.28 equal to zero leads to the equivalence of $\langle 103\rangle$ and $\langle 117\rangle$.

It can be deduced from (4.10) that

$$
\begin{aligned}
r\left(2 I_{m}-A B B^{\dagger} A^{\dagger}\right) & =r\left[\begin{array}{cc}
B^{*} B & B^{*} A^{\dagger} \\
A B & 2 I_{m}
\end{array}\right]-r(B)=r\left[\begin{array}{cc}
B^{*} B-B^{*} A^{\dagger} A B / 2 & 0 \\
0 & 2 I_{m}
\end{array}\right]-r(B) \\
& =m+r\left[B^{*}\left(I_{n}-A^{\dagger} A / 2\right) B\right]-r(B) \\
& =m+r(B)-r(B)=m, \\
r\left(2 I_{p}-B^{\dagger} A^{\dagger} A B\right) & =r\left[\begin{array}{cc}
A A^{*} & A B \\
B^{\dagger} A^{*} & 2 I_{p}
\end{array}\right]-r(A)=r\left[\begin{array}{cc}
A A^{*}-A B B^{\dagger} A^{*} / 2 & 0 \\
0 & 2 I_{p}
\end{array}\right]-r(A) \\
& =p+r\left[A\left(I_{p}-B B^{\dagger} / 2\right) A^{*}\right]-r(A) \\
& =p+r(A)-r(A)=p,
\end{aligned}
$$




$$
\begin{aligned}
r\left(2 I_{n}-A^{\dagger} A B B^{\dagger}\right) & =r\left(2 I_{n}-B B^{\dagger} A^{\dagger} A\right)=r\left[\begin{array}{cc}
B^{*} B & B^{*} A^{\dagger} A \\
B & 2 I_{n}
\end{array}\right]-r(B) \\
& =r\left[\begin{array}{cc}
B^{*} B-B^{*} A^{\dagger} A B / 2 & 0 \\
0 & 2 I_{n}
\end{array}\right]-r(B) \\
& =r\left[B^{*}\left(I_{n}-A^{\dagger} A / 2\right) B\right]+n-r(B) \\
& =r(B)+n-r(B)=n .
\end{aligned}
$$

We then derive from (4.13), (9.8)-(9.10), and 9.29- 9.31) the following formulas

$$
\begin{aligned}
& r\left[\left(I_{m}-A B B^{\dagger} A^{\dagger}\right)^{\dagger}-\left(I_{m}-A B B^{\dagger} A^{\dagger}\right)\right]=r\left[\left(I_{m}-A B B^{\dagger} A^{\dagger}\right)-\left(I_{m}-A B B^{\dagger} A^{\dagger}\right)^{3}\right] \\
& =r\left(I_{m}-A B B^{\dagger} A^{\dagger}\right)+r\left(2 I_{m}-A B B^{\dagger} A^{\dagger}\right)+r\left(A B B^{\dagger} A^{\dagger}\right)-2 m \\
& =r(N)-r(A)-r(B)+2 m+r(A B)-2 m \\
& =r(N)-r(A)-r(B)+r(A B), \\
& r\left[\left(I_{p}-B^{\dagger} A^{\dagger} A B\right)^{\dagger}-\left(I_{p}-B^{\dagger} A^{\dagger} A B\right)\right]=r\left[\left(I_{p}-B^{\dagger} A^{\dagger} A B\right)-\left(I_{p}-B^{\dagger} A^{\dagger} A B\right)^{3}\right] \\
& =r\left(I_{p}-B^{\dagger} A^{\dagger} A B\right)+r\left(2 I_{p}-B^{\dagger} A^{\dagger} A B\right)+r\left(B^{\dagger} A^{\dagger} A B\right)-2 p \\
& =r(N)-r(A)-r(B)+2 p+r(A B)-2 p \\
& =r(N)-r(A)-r(B)+r(A B), \\
& r\left[\left(I_{n}-A^{\dagger} A B B^{\dagger}\right)^{\dagger}-\left(I_{n}-A^{\dagger} A B B^{\dagger}\right)\right]=r\left[\left(I_{n}-A^{\dagger} A B B^{\dagger}\right)-\left(I_{n}-A^{\dagger} A B B^{\dagger}\right)^{3}\right] \\
& =r\left(I_{n}-A^{\dagger} A B B^{\dagger}\right)+r\left(2 I_{n}-A^{\dagger} A B B^{\dagger}\right)+r\left(A^{\dagger} A B B^{\dagger}\right)-2 n \\
& =r(N)-r(A)-r(B)+2 n+r(A B)-2 n \\
& =r(N)-r(A)-r(B)+r(A B) .
\end{aligned}
$$

Setting all sides of $\left(9.32-9.34\right.$ equal to zero leads to the equivalence of $\langle 104\rangle,\langle 105\rangle$, and $\langle 116\rangle$. Replacing $A^{\dagger} A$ with $F_{A}$ and $B B^{\dagger}$ with $E_{B}$ in $\langle 104\rangle$ and $\langle 105\rangle$ respectively leads to the equivalence of $\langle 106\rangle-\langle 109\rangle$ and $\langle 117\rangle-\langle 119\rangle$.

The following rank identities

$$
\begin{aligned}
r\left(N N^{\dagger}-A^{\dagger} A-B B^{\dagger}+A^{\dagger} A B B^{\dagger}\right) & =r\left(N N^{\dagger}-A^{\dagger} A-B B^{\dagger}+B B^{\dagger} A^{\dagger} A\right) \\
& =r(N)-r(A)-r(B)+r(A B)
\end{aligned}
$$

were proved in 102 . Setting the three sides of these two identities equal to zero leads to the equivalence of $\langle 110\rangle$ and $\langle 116\rangle$. Replacing $A^{*}$ and $B$ with $F_{A}$ and $E_{B}$ in $\langle 110\rangle$ respectively leads to the equivalence of $\langle 111\rangle-\langle 113\rangle$ and $\langle 117\rangle-\langle 119\rangle$.

It follows from (4.2) and (4.3) that

$$
\begin{aligned}
& r\left(A^{\dagger} A-A^{\dagger} A B B^{\dagger}\right)=r\left(E_{B} A^{*}\right)=r(N)-r(B), \\
& r\left(B B^{\dagger}-A^{\dagger} A B B^{\dagger}\right)=r\left(F_{A} B\right)=r(N)-r(A) .
\end{aligned}
$$

Thus the two rank equalities in $\langle 126\rangle$ are equivalent to $\langle 116\rangle$. Replacing $A^{\dagger} A$ and $B B^{\dagger}$ with $F_{A}$ and $E_{B}$ in $\langle 126\rangle$ respectively leads to the equivalence of $\langle 127\rangle-\langle 129\rangle$ and $\langle 117\rangle-\langle 119\rangle$.

The equivalence of $\langle 120\rangle-\langle 125\rangle$ and $\langle 130\rangle-\langle 137\rangle$ respectively follows from Lemma 4.4(f). The equivalence of $\langle 130\rangle-\langle 137\rangle$ and $\langle 138\rangle-\langle 145\rangle$ are obvious.

By (4.8),

$$
\begin{aligned}
& \operatorname{dim}\left[\mathscr{R}\left(A^{*}\right) \cap \mathscr{R}(B)\right]=r(A)+r(B)-r\left[A^{*}, B\right], \\
& \operatorname{dim}\left[\mathscr{R}\left(F_{A}\right) \cap \mathscr{R}(B)\right]=r\left(F_{A}\right)+r(B)-r\left[F_{A}, B\right], \\
& \operatorname{dim}\left[\mathscr{R}\left(A^{*}\right) \cap \mathscr{R}\left(E_{B}\right)\right]=r(A)+r\left(E_{B}\right)-r\left[A^{*}, E_{B}\right], \\
& \operatorname{dim}\left[\mathscr{R}\left(F_{A}\right) \cap \mathscr{R}\left(E_{B}\right)\right]=r\left(F_{A}\right)+r\left(E_{B}\right)-r\left[F_{A}, E_{B}\right] .
\end{aligned}
$$

Thus the equivalence of $\langle 146\rangle$ and $\langle 116\rangle-\langle 119\rangle$ follows.

Applying Lemma $4.4(\mathrm{a})$ and (b) to $\langle 89\rangle$ leads to the equivalence of $\langle 89\rangle$ and $\langle 149\rangle$. Replacing $A^{\dagger} A$ and $B B^{\dagger}$ with $F_{A}$ and $E_{B}$ in $\langle 149\rangle$ respectively leads to the equivalence of $\langle 148\rangle-\langle 150\rangle$ and $\langle 117\rangle-\langle 119\rangle$.

It follows first from $\langle 147\rangle$ that

$$
\mathscr{R}\left(A^{\dagger} A B B^{\dagger}\right) \subseteq \mathscr{R}\left(A^{\dagger} A\right) \cap \mathscr{R}\left(B B^{\dagger}\right)
$$

and/or

$$
\mathscr{R}\left(B B^{\dagger} A^{\dagger} A\right) \subseteq \mathscr{R}\left(A^{\dagger} A\right) \cap \mathscr{R}\left(B B^{\dagger}\right),
$$


and from $\langle 116\rangle$ that

$$
\begin{aligned}
\operatorname{dim}\left[\mathscr{R}\left(A^{\dagger} A\right) \cap \mathscr{R}\left(B B^{\dagger}\right)\right] & =r\left(A^{\dagger} A\right)+r\left(B B^{\dagger}\right)-r\left[A^{\dagger} A, B B^{\dagger}\right] \\
& =r\left(A^{\dagger} A B B^{\dagger}\right)=r\left(B B^{\dagger} A^{\dagger} A\right) .
\end{aligned}
$$

Applying (8.18) to (9.35), 9.36), and (9.37) leads to the range identities in $\langle 151\rangle$. Conversely, $\langle 151\rangle$ obviously implies $\langle 147\rangle$. Replacing $A^{\dagger} A$ and $B B^{\dagger}$ with $F_{A}$ and $E_{B}$ in $\langle 151\rangle$ respectively leads to the equivalence of $\langle 152\rangle-\langle 154\rangle$ and $\langle 117\rangle-\langle 119\rangle$.

By 4.25 ,

$$
r\left(A^{\dagger} A B B^{\dagger}-B B^{\dagger} A^{\dagger} A\right)=2 r\left[A^{\dagger} A B B^{\dagger}, B B^{\dagger} A^{\dagger} A\right]-2 r\left(B B^{\dagger} A^{\dagger} A\right) .
$$

Setting both sides of $(9.38)$ equal to zero leads to the equivalence of the first range equality in $\langle 91\rangle$ and $\langle 155\rangle$. Replacing $A^{\dagger} A$ and $B B^{\dagger}$ with $F_{A}$ and $E_{B}$ in the first range equality of $\langle 155\rangle$ respectively leads to the equivalences of the second, third, and fourth range equalities in $\langle 155\rangle$ and those in $\langle 117\rangle-\langle 119\rangle$.

By (4.8), the dimension of the intersection $\mathscr{R}(A B) \cap \mathscr{R}\left(A E_{B}\right)$ is reduced to

$$
\begin{aligned}
\operatorname{dim}\left[\mathscr{R}(A B) \cap \mathscr{R}\left(A E_{B}\right)\right] & =r(A B)+r\left(A E_{B}\right)-r\left[A B, A E_{B}\right] \\
& =r(A B)+r(N)-r(B)-r[A B, A] \\
& =r(N)-r(A)-r(B)+r(A B) .
\end{aligned}
$$

Setting both sides of $(9.39)$ equal to zero, we obtain the equivalence of the first range equality in $\langle 156\rangle$ and $\langle 116\rangle$. The equivalence of the other three range equalities in $\langle 128\rangle$ with $\langle 116\rangle-\langle 119\rangle$ can be shown similarly.

The equivalence of $\langle 156\rangle$ and $\langle 157\rangle$ follows from the definition of direct sum of linear subspaces.

By (4.8) and 4.23),

$$
\begin{aligned}
& \operatorname{dim}\left[\mathscr{R}\left(A^{\dagger} A-B B^{\dagger}\right) \cap \mathscr{R}\left(A^{\dagger} A+B B^{\dagger}-I_{n}\right)\right] \\
& =r\left(A^{\dagger} A-B B^{\dagger}\right)+r\left(A^{\dagger} A+B B^{\dagger}-I_{n}\right)-r\left[A^{\dagger} A-B B^{\dagger}, A^{\dagger} A+B B^{\dagger}-I_{n}\right] \\
& =r\left(A^{\dagger} A-B B^{\dagger}\right)+r\left(A^{\dagger} A+B B^{\dagger}-I_{n}\right)-r\left[A^{\dagger} A-B B^{\dagger}, 2 A^{\dagger} A-I_{n}\right] \\
& =r\left(A^{\dagger} A-B B^{\dagger}\right)+r\left(A^{\dagger} A+B B^{\dagger}-I_{n}\right)-r\left[A^{\dagger} A-B B^{\dagger}, I_{n}\right] \\
& =r\left(A^{\dagger} A-B B^{\dagger}\right)+r\left(A^{\dagger} A+B B^{\dagger}-I_{n}\right)-n \\
& =r\left(A^{\dagger} A B B^{\dagger}-B B^{\dagger} A^{\dagger} A\right) .
\end{aligned}
$$

Setting all sides of 9.40$\rangle$ equal to zero leads to the equivalence of the first equality in $\langle 91\rangle$ and $\langle 158\rangle$.

The equivalence of $\langle 91\rangle$ and $\langle 159\rangle-\langle 161\rangle$ follows from Lemma 4.14(a)-(e).

Applying 5.2 to $\langle 147\rangle-\langle 150\rangle$ leads to the equivalence of $\langle 147\rangle-\langle 150\rangle$ and $\langle 162\rangle-\langle 165\rangle$.

The commutativity of the product of two orthogonal projectors is a quite special topic that attracts much attention in matrix theory, and has certain important applications in mathematics. Many results on the commutativity problem were scattered in the literature; see e.g., $2,6,9,13,17,36,39,53,61,62,78,103$.

Theorem 9.2 ( [41]). Let $A \in \mathbb{C}^{m \times n}$ and $B \in \mathbb{C}^{n \times p}$, and denote $N=\left[A^{*}, B\right]$. Then the following 28 statements are equivalent:
$\langle 1\rangle \quad\left\{(A B)^{(1)}\right\} \supseteq\left\{B^{(1,2,4)} A^{(1,2,3)}\right\}$.
$\langle 3\rangle \quad\left\{(A B)^{(1)}\right\} \supseteq\left\{B^{(1,2,4)} A^{(1,2)}\right\}$.
$\langle 5\rangle \quad\left\{(A B)^{(1)}\right\} \supseteq\left\{B^{(1,4)} A^{(1,2,3)}\right\}$.
$\langle 7\rangle \quad\left\{(A B)^{(1)}\right\} \supseteq\left\{B^{(1,4)} A^{(1,2)}\right\}$.
$\langle 9\rangle \quad\left\{(A B)^{(1)}\right\} \supseteq\left\{B^{(1,2)} A^{(1,2,3)}\right\}$.
$\langle 11\rangle \quad\left\{(A B)^{(1)}\right\} \supseteq\left\{B^{(1,2)} A^{(1,2)}\right\}$.
$\langle 13\rangle \quad\left\{(A B)^{(1)}\right\} \supseteq\left\{B^{(1)} A^{(1,2,3)}\right\}$.
$\langle 15\rangle \quad\left\{(A B)^{(1)}\right\} \supseteq\left\{B^{(1)} A^{(1,2)}\right\}$.
$\langle 17\rangle \quad\left\{A B(A B)^{(1)}\right\} \supseteq\left\{A B B^{(1)} A^{(1,2,3)}\right\}$.
$\langle 19\rangle \quad\left\{A B(A B)^{(1)}\right\} \supseteq\left\{A B B^{(1)} A^{(1,2)}\right\}$.
$\langle 21\rangle \quad\left\{(A B)^{(1)} A B\right\} \supseteq\left\{B^{(1,2,4)} A^{(1)} A B\right\}$.
$\langle 23\rangle \quad\left\{(A B)^{(1)} A B\right\} \supseteq\left\{B^{(1,2)} A^{(1)} A B\right\}$.

$\langle 2\rangle \quad\left\{(A B)^{(1)}\right\} \supseteq\left\{B^{(1,2,4)} A^{(1,3)}\right\}$.

$\langle 4\rangle \quad\left\{(A B)^{(1)}\right\} \supseteq\left\{B^{(1,2,4)} A^{(1)}\right\}$.

$\langle 6\rangle \quad\left\{(A B)^{(1)}\right\} \supseteq\left\{B^{(1,4)} A^{(1,3)}\right\}$.

$\langle 8\rangle \quad\left\{(A B)^{(1)}\right\} \supseteq\left\{B^{(1,4)} A^{(1)}\right\}$.

$\langle 11\rangle \quad\left\{(A B)^{(1)}\right\} \supseteq\left\{B^{(1,2)} A^{(1,3)}\right\}$.

$\langle 12\rangle \quad\left\{(A B)^{(1)}\right\} \supseteq\left\{B^{(1,2)} A^{(1)}\right\}$.

$\langle 14\rangle \quad\left\{(A B)^{(1)}\right\} \supseteq\left\{B^{(1)} A^{(1,3)}\right\}$.

$\langle 16\rangle \quad\left\{(A B)^{(1)}\right\} \supseteq\left\{B^{(1)} A^{(1)}\right\}$.

$\langle 18\rangle \quad\left\{A B(A B)^{(1)}\right\} \supseteq\left\{A B B^{(1)} A^{(1,3)}\right\}$.

$\langle 20\rangle \quad\left\{A B(A B)^{(1)}\right\} \supseteq\left\{A B B^{(1)} A^{(1)}\right\}$.

$\langle 22\rangle \quad\left\{(A B)^{(1)} A B\right\} \supseteq\left\{B^{(1,4)} A^{(1)} A B\right\}$.

$\langle 24\rangle \quad\left\{(A B)^{(1)} A B\right\} \supseteq\left\{B^{(1)} A^{(1)} A B\right\}$.

$\langle 25\rangle \quad\left\{B(A B)^{(1)} A\right\} \supseteq\left\{B B^{(1)} A^{(1)} A\right\}$.

$\langle 26\rangle$ Either $A B=0$ or $r(A B)=r(A)+r(B)-n$.

$\langle 27\rangle$ Either $A B=0$ or $F_{A} E_{B}=0$.

$\langle 28\rangle$ Either $\mathscr{N}(A) \supseteq \mathscr{R}(B)$ or $\mathscr{R}\left(A^{*}\right) \subseteq \mathscr{N}\left(B^{*}\right)$. 
Proof. The equivalence of $\langle 1\rangle-\langle 16\rangle,\langle 26\rangle,\langle 27\rangle$, and $\langle 28\rangle$ follows from 8.30 and Lemma 8.6(f). The equivalence of $\langle 1\rangle$ and $\langle 17\rangle-\langle 25\rangle$ follows from definitions.

Some well-known results on the reverse order law $(A B)^{(1)}=B^{(1)} A^{(1)}$ can also be found in [65,66, 106]. We next characterize the set inclusions in (1.9) for $\{1,2\}-,\{1,3\}-$, and $\{1,4\}$-generalized inverses of $A B$.

Theorem 9.3. Let $A \in \mathbb{C}^{m \times n}$ and $B \in \mathbb{C}^{n \times p}$ be given, and denote $M=A B, N=\left[A^{*}, B\right]$ and $t=m+p+r(M)-$ $r(A)-r(B)$. Then the following results hold.

(a) The following 5 statements are equivalent:
$\langle 1\rangle \quad\left\{(A B)^{(1,2)}\right\} \ni B^{\dagger} A^{\dagger}$.
$\langle 3\rangle \quad\left\{(A B)^{(1,2)}\right\} \supseteq\left\{B^{(1,2,3)} A^{\dagger}\right\}$.
<5 $A B B^{\dagger} A^{\dagger} A B=A B$ and $/$ or $B^{\dagger} A^{\dagger} A B B^{\dagger} A^{\dagger}=B^{\dagger} A^{\dagger}$.
$\langle 2\rangle \quad\left\{(A B)^{(1,2)}\right\} \supseteq\left\{B^{\dagger} A^{(1,2,4)}\right\}$.
$\langle 6\rangle \quad r(M)=r(A)+r(B)-r(N)$.

(b) The following 9 statements are equivalent:
$\langle 1\rangle \quad\left\{(A B)^{(1,2)}\right\} \supseteq\left\{B^{\dagger} A^{(1,2,3)}\right\}$.
$\langle 3\rangle \quad\left\{(A B)^{(1,2)}\right\} \supseteq\left\{B^{(1,2,4)} A^{\dagger}\right\}$.
$\langle 2\rangle \quad\left\{(A B)^{(1,2)}\right\} \supseteq\left\{B^{\dagger} A^{(1,2)}\right\}$.
$\langle 5\rangle \quad\left\{(A B)^{(1,2)}\right\} \supseteq\left\{B^{(1,2,3)} A^{(1,2,3)}\right\}$.
$\langle 7\rangle \quad\left\{(A B)^{(1,2)}\right\} \supseteq\left\{B^{(1,2)} A^{\dagger}\right\}$.
$\langle 4\rangle \quad\left\{(A B)^{(1,2)}\right\} \supseteq\left\{B^{(1,2,4)} A^{(1,2,4)}\right\}$.
$\langle 6\rangle \quad\left\{(A B)^{(1,2)}\right\} \supseteq\left\{B^{(1,2,3)} A^{(1,2)}\right\}$.
$\langle 9\rangle \quad$ Either $\mathscr{R}\left(A^{*}\right) \subseteq \mathscr{R}(B)$ or $\mathscr{R}\left(A^{*}\right) \supseteq \mathscr{R}(B)$.

(c) The following 9 statements are equivalent:
$\langle 1\rangle \quad\left\{(A B)^{(1,2)}\right\} \supseteq\left\{B^{(1,3,4)} A^{(1,2,3)}\right\}$.
$\langle 2\rangle \quad\left\{(A B)^{(1,2)}\right\} \supseteq\left\{B^{(1,3,4)} A^{(1,2)}\right\}$
$\langle 3\rangle \quad\left\{(A B)^{(1,2)}\right\} \supseteq\left\{B^{(1,4)} A^{\dagger}\right\}$.
$\langle 4\rangle \quad\left\{(A B)^{(1,2)}\right\} \supseteq\left\{B^{(1,4)} A^{(1,2,4)}\right\}$.
$\langle 5\rangle \quad\left\{(A B)^{(1,2)}\right\} \supseteq\left\{B^{(1,3)} A^{(1,2,3)}\right\}$.
$\langle 6\rangle \quad\left\{(A B)^{(1,2)}\right\} \supseteq\left\{B^{(1,3)} A^{(1,2)}\right\}$.
$\langle 7\rangle \quad\left\{(A B)^{(1,2)}\right\} \supseteq\left\{B^{(1)} A^{\dagger}\right\}$.
$\langle 8\rangle \quad\left\{(A B)^{(1,2)}\right\} \supseteq\left\{B^{(1)} A^{(1,2,4)}\right\}$.
$\langle 9\rangle \quad$ Either $\mathscr{R}\left(A^{*}\right) \subseteq \mathscr{R}(B)$ or $\left\{\mathscr{R}\left(A^{*}\right) \supseteq \mathscr{R}(B)\right.$ and $\left.r(B)=p\right\}$.

(d) The following 9 statements are equivalent:
$\langle 1\rangle \quad\left\{(A B)^{(1,2)}\right\} \supseteq\left\{B^{\dagger} A^{(1,3)}\right\}$.
$\langle 3\rangle \quad\left\{(A B)^{(1,2)}\right\} \supseteq\left\{B^{(1,2,4)} A^{(1,3,4)}\right\}$.
$\langle 5\rangle \quad\left\{(A B)^{(1,2)}\right\} \supseteq\left\{B^{(1,2,3)} A^{(1,3)}\right\}$.
$\langle 2\rangle \quad\left\{(A B)^{(1,2)}\right\} \supseteq\left\{B^{\dagger} A^{(1)}\right\}$.
$\langle 7\rangle \quad\left\{(A B)^{(1,2)}\right\} \supseteq\left\{B^{(1,2)} A^{(1,3,4)}\right\}$.
$\langle 4\rangle \quad\left\{(A B)^{(1,2)}\right\} \supseteq\left\{B^{(1,2,4)} A^{(1,4)}\right\}$.
$\langle 9\rangle \quad$ Either $\mathscr{R}\left(A^{*}\right) \supseteq \mathscr{R}(B)$ or $\left\{r(A)=m\right.$ and $\left.\mathscr{R}\left(A^{*}\right) \subseteq \mathscr{R}(B)\right\}$.
$\langle 6\rangle \quad\left\{(A B)^{(1,2)}\right\} \supseteq\left\{B^{(1,2,3)} A^{(1)}\right\}$.

(e) The following 5 statements are equivalent:
$\langle 1\rangle \quad\left\{(A B)^{(1,2)}\right\} \supseteq\left\{B^{(1,3,4)} A^{\dagger}\right\}$.
$\langle 3\rangle \quad\left\{(A B)^{(1,2)}\right\} \supseteq\left\{B^{(1,3)} A^{\dagger}\right\}$.
$\langle 2\rangle \quad\left\{(A B)^{(1,2)}\right\} \supseteq\left\{B^{(1,3,4)} A^{(1,2,4)}\right\}$.
$\langle 5\rangle \quad$ Either $\mathscr{R}\left(A^{*}\right) \subseteq \mathscr{R}(B)$ or $\{r(B)=p$ and $r(M)=r(A)+r(B)-r(N)\}$.
$\langle 4\rangle \quad\left\{(A B)^{(1,2)}\right\} \supseteq\left\{B^{(1,3)} A^{(1,2,4)}\right\}$.

(f) The following 5 statements are equivalent:
$\langle 1\rangle \quad\left\{(A B)^{(1,2)}\right\} \supseteq\left\{B^{\dagger} A^{(1,3,4)}\right\}$.
$\langle 2\rangle \quad\left\{(A B)^{(1,2)}\right\} \supseteq\left\{B^{\dagger} A^{(1,4)}\right\}$.
$\langle 3\rangle \quad\left\{(A B)^{(1,2)}\right\} \supseteq\left\{B^{(1,2,3)} A^{(1,3,4)}\right\}$.
$\langle 4\rangle \quad\left\{(A B)^{(1,2)}\right\} \supseteq\left\{B^{(1,2,3)} A^{(1,4)}\right\}$.
$\langle 5\rangle \quad$ Either $\mathscr{R}\left(A^{*}\right) \supseteq \mathscr{R}(B)$ or $\{r(A)=m$ and $r(M)=r(A)+r(B)-r(N)\}$.

(g) The following 9 statements are equivalent:
$\langle 1\rangle \quad\left\{(A B)^{(1,2)}\right\} \supseteq\left\{B^{(1,3,4)} A^{(1,3)}\right\}$.
$\langle 3\rangle \quad\left\{(A B)^{(1,2)}\right\} \supseteq\left\{B^{(1,4)} A^{(1,3,4)}\right\}$.
$\langle 2\rangle \quad\left\{(A B)^{(1,2)}\right\} \supseteq\left\{B^{(1,3,4)} A^{(1)}\right\}$.
$\langle 5\rangle \quad\left\{(A B)^{(1,2)}\right\} \supseteq\left\{B^{(1,3)} A^{(1,3)}\right\}$.
$\langle 4\rangle \quad\left\{(A B)^{(1,2)}\right\} \supseteq\left\{B^{(1,4)} A^{(1,4)}\right\}$.
$\langle 7\rangle \quad\left\{(A B)^{(1,2)}\right\} \supseteq\left\{B^{(1)} A^{(1,3,4)}\right\}$.
$\langle 6\rangle \quad\left\{(A B)^{(1,2)}\right\} \supseteq\left\{B^{(1,3)} A^{(1)}\right\}$.
$\langle 9\rangle \quad r(M)=r(A)+r(B)-r(N)=\min \{m, n, p\}$. 
(h) The following 5 statements are equivalent:
$\langle 1\rangle \quad\left\{(A B)^{(1,2)}\right\} \supseteq\left\{B^{(1,3,4)} A^{(1,3,4)}\right\}$.
$\langle 3\rangle \quad\left\{(A B)^{(1,2)}\right\} \supseteq\left\{B^{(1,3)} A^{(1,3,4)}\right\}$.
$\langle 2\rangle \quad\left\{(A B)^{(1,2)}\right\} \supseteq\left\{B^{(1,3,4)} A^{(1,4)}\right\}$.
$\langle 5\rangle \quad r(M)=r(A)+r(B)-r(N)=\min \{m, n, p, t\}$.

(i) The following 5 statements are equivalent:
$\langle 1\rangle \quad\left\{(A B)^{(1,2)}\right\} \supseteq\left\{B^{(1,2,4)} A^{(1,2,3)}\right\}$.
$\langle 2\rangle \quad\left\{(A B)^{(1,2)}\right\} \supseteq\left\{B^{(1,2,4)} A^{(1,2)}\right\}$.
$\langle 3\rangle \quad\left\{(A B)^{(1,2)}\right\} \supseteq\left\{B^{(1,2)} A^{(1,2,3)}\right\}$.
$\langle 4\rangle \quad\left\{(A B)^{(1,2)}\right\} \supseteq\left\{B^{(1,2)} A^{(1,2)}\right\}$.
$\langle 5\rangle \quad A=0$ or $B=0$ or $r(A)=n$ or $r(B)=n$.

(j) The following 5 statements are equivalent:
$\langle 1\rangle \quad\left\{(A B)^{(1,2)}\right\} \supseteq\left\{B^{(1,2,4)} A^{(1,3)}\right\}$.
$\langle 2\rangle \quad\left\{(A B)^{(1,2)}\right\} \supseteq\left\{B^{(1,2,4)} A^{(1)}\right\}$.
$\langle 3\rangle \quad\left\{(A B)^{(1,2)}\right\} \supseteq\left\{B^{(1,2)} A^{(1,3)}\right\}$.
$\langle 4\rangle \quad\left\{(A B)^{(1,2)}\right\} \supseteq\left\{B^{(1,2)} A^{(1)}\right\}$.
$\langle 5\rangle \quad r(A)=n$ or $B=0$ or $\{r(A)=m$ and $r(B)=n\}$.

(k) The following 5 statements are equivalent:
$\langle 1\rangle \quad\left\{(A B)^{(1,2)}\right\} \supseteq\left\{B^{(1,4)} A^{(1,2,3)}\right\}$.
$\langle 2\rangle \quad\left\{(A B)^{(1,2)}\right\} \supseteq\left\{B^{(1,4)} A^{(1,2)}\right\}$.
$\langle 3\rangle \quad\left\{(A B)^{(1,2)}\right\} \supseteq\left\{B^{(1)} A^{(1,2,3)}\right\}$.
$\langle 4\rangle \quad\left\{(A B)^{(1,2)}\right\} \supseteq\left\{B^{(1)} A^{(1,2)}\right\}$.
$\langle 5\rangle \quad A=0$ or $r(B)=n$ or $\{r(A)=n$ and $r(B)=p\}$.

(1) The following 5 statements are equivalent:
$\langle 1\rangle \quad\left\{(A B)^{(1,2)}\right\} \supseteq\left\{B^{(1,4)} A^{(1,3)}\right\}$.
$\langle 3\rangle \quad\left\{(A B)^{(1,2)}\right\} \supseteq\left\{B^{(1)} A^{(1,3)}\right\}$.
$\langle 2\rangle \quad\left\{(A B)^{(1,2)}\right\} \supseteq\left\{B^{(1,4)} A^{(1)}\right\}$.
$\langle 4\rangle \quad\left\{(A B)^{(1,2)}\right\} \supseteq\left\{B^{(1)} A^{(1)}\right\}$.
$\langle 5\rangle \quad\{r(A)=m$ and $r(B)=n\}$ or $\{r(A)=r(B)=n\}$ or $\{r(A)=n$ and $r(B)=p\}$.

Proof. Applying (8.25) to $B^{\dagger} A^{\dagger} A B B^{\dagger} A^{\dagger}-B^{\dagger} A^{\dagger}$ and simplifying by Lemma 8.5, we obtain

$$
\begin{aligned}
r\left(B^{\dagger} A^{\dagger} A B B^{\dagger} A^{\dagger}-B^{\dagger} A^{\dagger}\right) & =r\left[\left(B^{\dagger}\right)^{*}, A^{\dagger}\right]-r\left(B^{\dagger} A^{\dagger}\right)-r\left(A^{\dagger}\right)-r\left(B^{\dagger}\right) \\
& =r(N)+r(M)-r(A)-r(B) .
\end{aligned}
$$

Setting both sides of 9.41 equal to zero leads to the equivalence of the second rank equality in $\langle 5\rangle$ and $\langle 6\rangle$ of (a). All the remaining equivalences in (a)-(l) follow from Lemma 3.3(b), Lemma 8.3, Theorem 9.1, as well as the simplification of the combined conditions.

Theorem 9.4. Let $A \in \mathbb{C}^{m \times n}$ and $B \in \mathbb{C}^{n \times p}$ be given, and denote $M=A B$ and $N=\left[A^{*}, B\right]$. Then the following results hold.

(a) The following 26 statements are equivalent:
$\langle 1\rangle \quad\left\{M^{(1,3)}\right\} \ni B^{\dagger} A^{\dagger}$.
$\langle 3\rangle \quad\left\{M^{(1,3)}\right\} \supseteq\left\{B^{(1,2,3)} A^{\dagger}\right\}$.
〈5) $M M^{\dagger}=M B^{\dagger} A^{\dagger}$.
$\langle 7\rangle \quad A B B^{\dagger} A^{*} M=A A^{*} M$.
$\langle 9\rangle \quad\left(A B B^{\dagger}\right)^{\dagger}=B B^{\dagger} A^{\dagger}$.
$\langle 11\rangle \quad\left(A E_{B}\right)^{\dagger}=E_{B} A^{\dagger}$.
$\langle 13\rangle \quad M M^{\dagger} A=M B^{\dagger}$.
$\langle 15\rangle \quad r\left[A^{*} M, B\right]=r(B)$.
$\langle 17\rangle \quad \mathscr{R}\left(A^{*} M\right) \subseteq \mathscr{R}(B)$.
$\langle 19\rangle \quad \mathscr{R}\left(A^{*} M\right)=\mathscr{R}\left(A^{*}\right) \cap \mathscr{R}(B)$.
$\langle 2\rangle \quad\left\{M^{(1,3)}\right\} \supseteq\left\{B^{(1,3,4)} A^{\dagger}\right\}$.
$\langle 4\rangle \quad\left\{M^{(1,3)}\right\} \supseteq\left\{B^{(1,3)} A^{\dagger}\right\}$.
$\langle 6\rangle \quad M^{*} M B^{\dagger} A^{\dagger}=M^{*}$.
〈8) $B B^{\dagger} A^{*} M=A^{*} M$.
$\langle 10\rangle \quad B^{*}\left(A B B^{\dagger}\right)^{\dagger} A A^{*}=M^{*}$.
$\langle 12\rangle \quad A\left(A E_{B}\right)^{\dagger}=A E_{B} A^{\dagger}$.
<14 $\quad A^{*} A B B^{\dagger}=B B^{\dagger} A^{*} A$.
$\langle 16\rangle \quad r\left[A^{*} A E_{B}, E_{B}\right]=r\left(E_{B}\right)$.
$\langle 18\rangle \quad \mathscr{R}\left(A^{*} A E_{B}\right) \subseteq \mathscr{N}\left(B^{*}\right)$.
$\langle 20\rangle \quad \mathscr{R}\left(A^{*} A E_{B}\right)=\mathscr{R}\left(A^{*}\right) \cap \mathscr{N}\left(B^{*}\right)$.
$\langle 21\rangle \quad \mathscr{R}\left(A^{*} A B B^{\dagger}\right)=\mathscr{R}\left(B B^{\dagger} A^{*} A\right)$.
$\langle 22\rangle \quad \mathscr{R}\left(A^{*} A E_{B}\right)=\mathscr{R}\left(E_{B} A^{*} A\right)$.
$\langle 23\rangle M B^{\dagger} A^{\dagger}$ is an orthogonal projector, i.e., $\left(M B^{\dagger} A^{\dagger}\right)^{2}=M B^{\dagger} A^{\dagger}=\left(M B^{\dagger} A^{\dagger}\right)^{*}$.
$\langle 24\rangle \quad A E_{B} A^{\dagger}$ is an orthogonal projector, i.e., $\left(A E_{B} A^{\dagger}\right)^{2}=A E_{B} A^{\dagger}=\left(A E_{B} A^{\dagger}\right)^{*}$.
$\langle 25\rangle$ Both $M B^{\dagger} A^{\dagger} M=M$ and $\left(M B^{\dagger} A^{\dagger}\right)^{*}=M B^{\dagger} A^{\dagger}$.
$\langle 26\rangle \quad$ Both $r(N)=r(A)+r(B)-r(M)$ and $r\left[A A^{*} M, M\right]=r(M)$. 
(b) The following 6 statements are equivalent:
〈1) $\quad\left\{M^{(1,3)}\right\} \supseteq\left\{B^{\dagger} A^{(1,3,4)}\right\}$.
$\langle 2\rangle \quad\left\{M^{(1,3)}\right\} \supseteq\left\{B^{(1,3,4)} A^{(1,3,4)}\right\}$.
$\langle 3\rangle \quad\left\{M^{(1,3)}\right\} \supseteq\left\{B^{(1,2,3)} A^{(1,3,4)}\right\}$.
$\langle 4\rangle \quad\left\{M^{(1,3)}\right\} \supseteq\left\{B^{(1,3)} A^{(1,3,4)}\right\}$.
〈5) $\quad M^{*} M B^{\dagger} F_{A} U E_{A} \equiv M^{*}-M^{*} M B^{\dagger} A^{\dagger}$ for all $U$.
$\langle 6\rangle \quad \mathscr{R}\left(A^{*} M\right) \subseteq \mathscr{R}(B)$.

(c) The following 6 statements are equivalent:
$\langle 1\rangle \quad\left\{M^{(1,3)}\right\} \supseteq\left\{B^{\dagger} A^{(1,2,4)}\right\}$.
$\langle 2\rangle \quad\left\{M^{(1,3)}\right\} \supseteq\left\{B^{(1,3,4)} A^{(1,2,4)}\right\}$.
$\langle 3\rangle \quad\left\{M^{(1,3)}\right\} \supseteq\left\{B^{(1,2,3)} A^{(1,2,4)}\right\}$.
$\langle 4\rangle \quad\left\{M^{(1,3)}\right\} \supseteq\left\{B^{(1,3)} A^{(1,2,4)}\right\}$.
〈5) $\quad M^{*} M B^{\dagger} A^{\dagger} A U E_{A} \equiv M^{*}-M^{*} M B^{\dagger} A^{\dagger}$ for all $U$.
$\langle 6\rangle \quad$ Both $r(A)=m$ and $\mathscr{R}\left(A^{*} M\right) \subseteq \mathscr{R}(B)$.

(d) The following 6 statements are equivalent:
$\langle 1\rangle \quad\left\{M^{(1,3)}\right\} \supseteq\left\{B^{\dagger} A^{(1,2,3)}\right\}$.
$\langle 2\rangle \quad\left\{M^{(1,3)}\right\} \supseteq\left\{B^{(1,3,4)} A^{(1,2,3)}\right\}$.
$\langle 3\rangle \quad\left\{M^{(1,3)}\right\} \supseteq\left\{B^{(1,2,3)} A^{(1,2,3)}\right\}$.
$\langle 4\rangle \quad\left\{M^{(1,3)}\right\} \supseteq\left\{B^{(1,3)} A^{(1,2,3)}\right\}$.
〈5) $\quad M^{*} M B^{\dagger} F_{A} U A A^{\dagger} \equiv M^{*}-M^{*} M B^{\dagger} A^{\dagger}$ for all $U$.
$\langle 6\rangle \quad \mathscr{R}\left(A^{*} M\right) \subseteq \mathscr{R}(B)$.

(e) The following 6 statements are equivalent:
$\langle 1\rangle \quad\left\{M^{(1,3)}\right\} \supseteq\left\{B^{\dagger} A^{(1,4)}\right\}$.
$\langle 2\rangle \quad\left\{M^{(1,3)}\right\} \supseteq\left\{B^{(1,3,4)} A^{(1,4)}\right\}$.
$\langle 3\rangle \quad\left\{M^{(1,3)}\right\} \supseteq\left\{B^{(1,2,3)} A^{(1,4)}\right\}$.
$\langle 4\rangle \quad\left\{M^{(1,3)}\right\} \supseteq\left\{B^{(1,3)} A^{(1,4)}\right\}$.
〈5) $\quad M^{*} M B^{\dagger} U E_{A} \equiv M^{*}-M^{*} M B^{\dagger} A^{\dagger}$ for all $U$.
$\langle 6\rangle \quad$ Both $r(A)=m$ and $\mathscr{R}\left(A^{*} M\right) \subseteq \mathscr{R}(B)$.

(f) The following 6 statements are equivalent:
$\langle 1\rangle \quad\left\{M^{(1,3)}\right\} \supseteq\left\{B^{\dagger} A^{(1,3)}\right\}$.
$\langle 3\rangle \quad\left\{M^{(1,3)}\right\} \supseteq\left\{B^{(1,2,3)} A^{(1,3)}\right\}$.
$\langle 2\rangle \quad\left\{M^{(1,3)}\right\} \supseteq\left\{B^{(1,3,4)} A^{(1,3)}\right\}$.
〈5) $M^{*} M B^{\dagger} F_{A} U \equiv M^{*}-M^{*} M B^{\dagger} A^{\dagger}$ for all $U$.
$\langle 6\rangle \quad \mathscr{R}\left(A^{*} M\right) \subseteq \mathscr{R}(B)$.

(g) The following 6 statements are equivalent:
$\langle 1\rangle \quad\left\{M^{(1,3)}\right\} \supseteq\left\{B^{\dagger} A^{(1,2)}\right\}$.
$\langle 2\rangle \quad\left\{M^{(1,3)}\right\} \supseteq\left\{B^{(1,3,4)} A^{(1,2)}\right\}$.
$\langle 3\rangle \quad\left\{M^{(1,3)}\right\} \supseteq\left\{B^{(1,2,3)} A^{(1,2)}\right\}$.
$\langle 4\rangle \quad\left\{M^{(1,3)}\right\} \supseteq\left\{B^{(1,3)} A^{(1,2)}\right\}$.
$\langle 5\rangle \quad\left(M^{*} M B^{\dagger} A^{\dagger}+M^{*} M B^{\dagger} F_{A} U_{1}\right)\left(A A^{\dagger}+A U_{2} E_{A}\right) \equiv M^{*}$ for all $U_{1}$ and $U_{2}$.
$\langle 6\rangle \quad$ Both $r(A)=m$ and $\mathscr{R}\left(A^{*} M\right) \subseteq \mathscr{R}(B)$.

(h) The following 6 statements are equivalent:
$\langle 1\rangle \quad\left\{M^{(1,3)}\right\} \supseteq\left\{B^{\dagger} A^{(1)}\right\}$.
$\langle 3\rangle \quad\left\{M^{(1,3)}\right\} \supseteq\left\{B^{(1,2,3)} A^{(1)}\right\}$.
$\langle 2\rangle \quad\left\{M^{(1,3)}\right\} \supseteq\left\{B^{(1,3,4)} A^{(1)}\right\}$.
$\langle 5\rangle \quad M^{*} M B^{\dagger} F_{A} U_{1}+M^{*} M B^{\dagger} U_{2} E_{A} \equiv M^{*}-M^{*} M B^{\dagger} A^{\dagger}$ for all $U_{1}$ and $U_{2}$.
$\langle 6\rangle \quad$ Both $r(A)=m$ and $\mathscr{R}\left(A^{*} M\right) \subseteq \mathscr{R}(B)$.

(i) The following 6 statements are equivalent:
$\langle 1\rangle \quad\left\{M^{(1,3)}\right\} \supseteq\left\{B^{(1,2,4)} A^{\dagger}\right\}$.
$\langle 2\rangle \quad\left\{M^{(1,3)}\right\} \supseteq\left\{B^{(1,4)} A^{\dagger}\right\}$.
$\langle 3\rangle \quad\left\{M^{(1,3)}\right\} \supseteq\left\{B^{(1,2)} A^{\dagger}\right\}$.
$\langle 4\rangle \quad\left\{M^{(1,3)}\right\} \supseteq\left\{B^{(1)} A^{\dagger}\right\}$.
〈5) $\quad M^{*} M V E_{B} A^{\dagger} \equiv M^{*}-M^{*} M B^{\dagger} A^{\dagger}$ for all $V$.
$\langle 6\rangle \quad \mathscr{R}\left(A^{*}\right) \subseteq \mathscr{R}(B)$.

(j) The following 6 statements are equivalent:
$\langle 1\rangle \quad\left\{M^{(1,3)}\right\} \supseteq\left\{B^{(1,2,4)} A^{(1,3,4)}\right\}$.
$\langle 2\rangle \quad\left\{M^{(1,3)}\right\} \supseteq\left\{B^{(1,4)} A^{(1,3,4)}\right\}$.
$\langle 3\rangle \quad\left\{M^{(1,3)}\right\} \supseteq\left\{B^{(1,2)} A^{(1,3,4)}\right\}$.
$\langle 4\rangle \quad\left\{M^{(1,3)}\right\} \supseteq\left\{B^{(1)} A^{(1,3,4)}\right\}$.
$\langle 5\rangle \quad\left(M^{*} M B^{\dagger}+M^{*} M V E_{B}\right)\left(A^{\dagger}+F_{A} U E_{A}\right) \equiv M^{*}$ for all $U$ and $V$.
$\langle 6\rangle$ Either $r(B)=n$ or $\left\{r(A)=m\right.$ and $\left.\mathscr{R}\left(A^{*}\right) \subseteq \mathscr{R}(B)\right\}$. 
(k) The following 6 statements are equivalent:
$\langle 1\rangle \quad\left\{M^{(1,3)}\right\} \supseteq\left\{B^{(1,2,4)} A^{(1,2,4)}\right\}$.
$\langle 3\rangle \quad\left\{M^{(1,3)}\right\} \supseteq\left\{B^{(1,2)} A^{(1,2,4)}\right\}$.
$\langle 2\rangle \quad\left\{M^{(1,3)}\right\} \supseteq\left\{B^{(1,4)} A^{(1,2,4)}\right\}$.
$\langle 5\rangle \quad\left(M^{*} M B^{\dagger}+M^{*} M V E_{B}\right)\left(A^{\dagger}+A^{\dagger} A U E_{A}\right) \equiv M^{*}$ for all $U$ and $V$.
$\langle 6\rangle \quad$ Both $r(A)=m$ and $\mathscr{R}\left(A^{*}\right) \subseteq \mathscr{R}(B)$.

(1) The following 6 statements are equivalent:
〈1) $\left\{M^{(1,3)}\right\} \supseteq\left\{B^{(1,2,4)} A^{(1,2,3)}\right\}$.
$\langle 2\rangle \quad\left\{M^{(1,3)}\right\} \supseteq\left\{B^{(1,4)} A^{(1,2,3)}\right\}$.
$\langle 3\rangle \quad\left\{M^{(1,3)}\right\} \supseteq\left\{B^{(1,2)} A^{(1,2,3)}\right\}$.
〈4) $\left\{M^{(1,3)}\right\} \supseteq\left\{B^{(1)} A^{(1,2,3)}\right\}$.
$\langle 5\rangle \quad\left(M^{*} M B^{\dagger}+M^{*} M V E_{B}\right)\left(A^{\dagger}+F_{A} U A A^{\dagger}\right) \equiv M^{*}$ for all $U$ and $\bar{V}$.
$\langle 6\rangle \quad r(B)=n$.

(m) The following 6 statements are equivalent:
$\langle 1\rangle \quad\left\{M^{(1,3)}\right\} \supseteq\left\{B^{(1,2,4)} A^{(1,4)}\right\}$.
$\langle 3\rangle \quad\left\{M^{(1,3)}\right\} \supseteq\left\{B^{(1,2)} A^{(1,4)}\right\}$.
$\langle 2\rangle \quad\left\{M^{(1,3)}\right\} \supseteq\left\{B^{(1,4)} A^{(1,4)}\right\}$.
$\langle 5\rangle \quad\left(M^{*} M B^{\dagger}+M^{*} M V E_{B}\right)\left(A^{\dagger}+U E_{A}\right) \equiv M^{*}$ for all $U$ and $V$.
$\langle 6\rangle \quad$ Both $r(A)=m$ and $\mathscr{R}\left(A^{*}\right) \subseteq \mathscr{R}(B)$.

(n) The following 6 statements are equivalent:
$\langle 1\rangle \quad\left\{M^{(1,3)}\right\} \supseteq\left\{B^{(1,2,4)} A^{(1,3)}\right\}$.
$\langle 2\rangle \quad\left\{M^{(1,3)}\right\} \supseteq\left\{B^{(1,4)} A^{(1,3)}\right\}$.
$\langle 3\rangle \quad\left\{M^{(1,3)}\right\} \supseteq\left\{B^{(1,2)} A^{(1,3)}\right\}$.
$\langle 4\rangle \quad\left\{M^{(1,3)}\right\} \supseteq\left\{B^{(1)} A^{(1,3)}\right\}$.
$\langle 5\rangle \quad\left(M^{*} M B^{\dagger}+M^{*} M V E_{B}\right)\left(A^{\dagger}+F_{A} U\right) \equiv M^{*}$ for all $U$ and $V$.
$\langle 6\rangle \quad r(B)=n$.

(o) The following 6 statements are equivalent:
$\langle 1\rangle \quad\left\{M^{(1,3)}\right\} \supseteq\left\{B^{(1,2,4)} A^{(1,2)}\right\}$.
$\langle 3\rangle \quad\left\{M^{(1,3)}\right\} \supseteq\left\{B^{(1,2)} A^{(1,2)}\right\}$.
$\langle 2\rangle \quad\left\{M^{(1,3)}\right\} \supseteq\left\{B^{(1,4)} A^{(1,2)}\right\}$.
$\langle 5\rangle \quad\left(M^{*} M B^{\dagger}+M^{*} M V E_{B}\right)\left(A^{\dagger}+F_{A} U_{1}\right)\left(A A^{\dagger}+A U_{2} E_{A}\right) \equiv M^{*}$ for all $U_{1}$, and $U_{2}$, and $V$.
$\langle 6\rangle$ Both $r(A)=m$ and $r(B)=n$.

(p) The following 6 statements are equivalent:
$\langle 1\rangle \quad\left\{M^{(1,3)}\right\} \supseteq\left\{B^{(1,2,4)} A^{(1)}\right\}$.
$\langle 2\rangle \quad\left\{M^{(1,3)}\right\} \supseteq\left\{B^{(1,4)} A^{(1)}\right\}$.
$\langle 3\rangle \quad\left\{M^{(1,3)}\right\} \supseteq\left\{B^{(1,2)} A^{(1)}\right\}$.
$\langle 4\rangle \quad\left\{M^{(1,3)}\right\} \supseteq\left\{B^{(1)} A^{(1)}\right\}$.
$\langle 5\rangle \quad\left(M^{*} M B^{\dagger}+M^{*} M V E_{B}\right)\left(A^{\dagger}+F_{A} U_{1}+U_{2} E_{A}\right) \equiv M^{*}$ for all $U_{1}$, and $U_{2}$, and $V$.
$\langle 6\rangle \quad$ Both $r(A)=m$ and $r(B)=n$.

Proof. The equivalence of $\langle 1\rangle$ and $\langle 25\rangle$ in (a) follows from the definition of $\{1,3\}$-generalized inverses of a matrix. The equivalence of $\langle 1\rangle-\langle 6\rangle$ in (a) follows from $(3.65)$ and $(3.156)$.

Applying 4.12 and simplifying gives

$$
\begin{aligned}
r\left(M M^{\dagger}-M B^{\dagger} A^{\dagger}\right) & =r\left(M^{*}-M^{*} M B^{\dagger} A^{\dagger}\right)=r\left[M^{*} A A^{*}-M^{*} M B^{\dagger} A^{*}\right) \\
& =r\left(A A^{*} A B-A B B^{\dagger} A^{*} A B\right)=r\left[\begin{array}{cc}
B^{*} B & B^{*} A^{*} A B \\
A B & A A^{*} A B
\end{array}\right]-r(B) \\
& =r\left(\left[\begin{array}{c}
B^{*} \\
A
\end{array}\right]\left[B, A^{*} A B\right]\right)-r(B)=r\left(\left[\begin{array}{c}
B^{*} \\
B^{*} A^{*} A
\end{array}\right]\left[B, A^{*} A B\right]\right)-r(B) \\
& =r\left[A^{*} A B, B\right]-r(B) .
\end{aligned}
$$

Setting all sides of $(9.42)$ equal to zero leads to the equivalence of $\langle 5\rangle-\langle 7\rangle,\langle 15\rangle$, and $\langle 17\rangle$.

By 4.26,

$$
r\left(B B^{\dagger} A^{*} M-A^{*} M\right)=r\left(E_{B} A^{*} M\right)=r\left[A^{*} A B, B\right]-r(B) .
$$


Setting all sides of 9.43 equal to zero leads to the equivalence of $\langle 8\rangle$ and $\langle 15\rangle$.

By (4.2),

$$
r\left[A^{*} A E_{B}, E_{B}\right]-r\left(E_{B}\right)=r\left(B B^{\dagger} A^{*} A E_{B}\right)=r\left(B^{*} A^{*} A E_{B}\right)=r\left[A^{*} A B, B^{*}\right]-r(B) .
$$

Setting all sides of 9.44 equal to zero leads to the equivalence of $\langle 15\rangle,\langle 16\rangle$, and $\langle 18\rangle$.

It follows from $t_{5} \geq t_{6}$ in (4.29) that

$$
r\left[A^{*} A B, B\right]-r(B) \geqslant r(M)+r(M)-r(A)-r(B) \geqslant 0 .
$$

Also by 8.19 ,

$$
\mathscr{R}\left(A^{*} A B\right) \subseteq \mathscr{R}(B) \Rightarrow \mathscr{R}\left(A A^{*} A B\right) \subseteq \mathscr{R}(A B)
$$

Combining (9.45) and $(9.46)$ yields

$$
r\left[A^{*} A B, B\right]=r(B) \Rightarrow \text { both } r(M)=r(A)+r(B)-r(M) \text { and } r\left[A A^{*} A B, A B\right]=r(M) .
$$

So that $\langle 28\rangle$ implies $\langle 15\rangle$. Conversely, substituting the two rank equalities in $\langle 28\rangle$ into both sides of $t_{4} \geq t_{5}$ in (4.29) yields $r\left[A^{*} A B, B\right] \leqslant r(B)$, which in fact implies $r\left[A^{*} A B, B\right]=r(B)$. So that $\langle 15\rangle$ is equivalent to $\langle 26\rangle$.

Applying (4.10) to $\left(A B B^{\dagger}\right)^{\dagger}-B B^{\dagger} A^{\dagger}$ and simplifying yields

$$
\begin{aligned}
r\left[\left(A B B^{\dagger}\right)^{\dagger}-B B^{\dagger} A^{\dagger}\right] & =r\left[B^{*}\left(A B B^{\dagger}\right)^{\dagger} A A^{*}-B^{*} A^{*}\right] \\
& =r\left[\begin{array}{cc}
\left(A B B^{\dagger}\right)^{*}\left(A B B^{\dagger}\right)\left(A B B^{\dagger}\right)^{*} & \left(A B B^{\dagger}\right)^{*} A A^{*} \\
B^{*}\left(A B B^{\dagger}\right)^{*} & B^{*} A^{*}
\end{array}\right]-r\left(A B B^{\dagger}\right) \\
& =r\left[\begin{array}{cc}
B^{*} A^{*} A B B^{\dagger} A^{*} & B^{*} A^{*} A A^{*} \\
B^{*} A^{*} & B^{*} A^{*}
\end{array}\right]-r(M) \\
& =r\left[\begin{array}{cc}
0 & B^{*} A^{*} A A^{*}-B^{*} A^{*} A B B^{\dagger} A^{*} \\
B^{*} A^{*} & 0
\end{array}\right]-r(M) \\
& =r\left(A A^{*} A B-A B B^{\dagger} A^{*} A B\right) \\
& =r\left[A^{*} A B, B\right]-r(B) \quad(\text { by }(9.42)) .
\end{aligned}
$$

Setting all sides of $(9.48)$ equal to zero leads to the equivalence of $\langle 9\rangle,\langle 10\rangle$, and $\langle 15\rangle$.

Replace $B B^{\dagger}$ with $E_{B}$ in (9.48) and applying (9.44) yields

$$
r\left[\left(A E_{B}\right)^{\dagger}-E_{B} A^{\dagger}\right]=r\left[A^{*} A E_{B}, E_{B}\right]-r\left(E_{B}\right)=r\left[A^{*} A B, B^{*}\right]-r(B) .
$$

Setting all sides of 9.49 equal to zero leads to the equivalence of $\langle 11\rangle$ and $\langle 15\rangle$.

By (9.42) and (9.44),

$$
\begin{aligned}
r\left[A\left(A E_{B}\right)^{\dagger}-A E_{B} A^{\dagger}\right] & =r\left[\left(A E_{B}\right)\left(A E_{B}\right)^{\dagger}-\left(A E_{B}\right)\left(E_{B}\right)^{\dagger} A^{\dagger}\right] \\
& =r\left[A^{*} A E_{B}, E_{B}\right]-r\left(E_{B}\right) \\
& =r\left[A^{*} A B, B^{*}\right]-r(B) .
\end{aligned}
$$

Setting all sides of 9.50 equal to zero leads to the equivalence of $\langle 12\rangle$ and $\langle 15\rangle$.

By (4.2),

$$
\begin{aligned}
r\left(M M^{\dagger} A-M B^{\dagger}\right) & =r\left[M^{*} A-M^{*} A B B^{\dagger}\right] \\
& =r\left(E_{B} A^{*} M\right)=r\left[A^{*} M, B\right]-r(B) .
\end{aligned}
$$

Setting both sides of 9.51 equal to zero leads to the equivalence of $\langle 13\rangle$ and $\langle 15\rangle$.

By (4.26),

$$
\begin{aligned}
r\left(A^{*} A B B^{\dagger}-B B^{\dagger} A^{*} A\right) & =2 r\left[A^{*} A B B^{\dagger}, B B^{\dagger}\right]-2 r\left(B B^{\dagger}\right) \\
& =2 r\left[A^{*} A B, B\right]-2 r(B)
\end{aligned}
$$

Setting all sides of 9.52 equal to zero leads to the equivalence of $\langle 14\rangle$ and $\langle 15\rangle$.

It follows from $\langle 17\rangle$ that

$$
\mathscr{R}\left(A^{*} A B\right) \subseteq \mathscr{R}\left(A^{*}\right) \cap \mathscr{R}(B)
$$


and from (4.8) and $\langle 28\rangle$ that

$$
\operatorname{dim}\left[\mathscr{R}\left(A^{*}\right) \cap \mathscr{R}(B)\right]=r(A)+r(B)-r\left[A^{*}, B\right]=r(M) .
$$

Applying (8.18) to 9.53 and 9.54 leads to the range identities in $\langle 19\rangle$. Conversely, $\langle 19\rangle$ obviously implies $\langle 17\rangle$. The equivalence of $\langle 18\rangle$ and $\langle 20\rangle$ can be shown similarly.

Replace $B B^{\dagger}$ with $E_{B}$ in 9.55 and applying (9.44) yields

$$
\mathscr{R}\left(A^{*} A E_{B}\right)=\mathscr{R}\left(E_{B} A^{*} A\right) \Leftrightarrow r\left[A^{*} A E_{B}, E_{B}\right]=r\left(E_{B}\right)
$$

establishing the equivalence of $\langle 16\rangle$ and $\langle 22\rangle$.

Since $M M^{\dagger}$ is both idempotent and Hermitian, $\langle 5\rangle$ implies $\langle 23\rangle$. Conversely, the first equality in $\langle 23\rangle$ is equivalent to $M B^{\dagger} A^{\dagger} M=M$ by Theorem $9.1\langle 48\rangle$ and $\langle 93\rangle$. This equality together with the second equality in $\langle 23\rangle$ implies $\langle 1\rangle$.

Replace $B B^{\dagger}$ with $E_{B}$ in $\langle 23\rangle$ leads to the equivalence of $\langle 16\rangle$ and $\langle 24\rangle$.

Applying Lemma 8.5 and (4.2) to $\left[A^{*} A B B^{\dagger}, B B^{\dagger} A^{*} A\right]$ yields

$$
\begin{aligned}
r\left[A^{*} A B B^{\dagger}, B B^{\dagger} A^{*} A\right] & =r\left[A^{*} A B, B B^{\dagger} A^{*}\right]=r\left[A^{*} A B-B B^{\dagger} A^{*} A B, B B^{\dagger} A^{*}\right] \\
& =r\left[\left(I_{n}-B B^{\dagger}\right) A^{*} A B, B B^{\dagger} A^{*}\right] \\
& =r\left[\left(I_{n}-B B^{\dagger}\right) A^{*} A B\right]+r\left(B B^{\dagger} A^{*}\right) \\
& =r\left[A^{*} A B, B\right]+r(M)-r(B) .
\end{aligned}
$$

Hence,

$$
\begin{aligned}
& \mathscr{R}\left(A^{*} A B B^{\dagger}\right)=\mathscr{R}\left(B B^{\dagger} A^{*} A\right) \\
& \Leftrightarrow r\left[A^{*} A B B^{\dagger}, B B^{\dagger} A^{*} A\right]=r\left(A^{*} A B B^{\dagger}\right)=r\left(B B^{\dagger} A^{*} A\right)=r(M) \\
& \Leftrightarrow r\left[A^{*} A B, B\right]=r(B),
\end{aligned}
$$

establishing the equivalence of $\langle 15\rangle$ and $\langle 21\rangle$. Replace $B B^{\dagger}$ with $E_{B}$ in $\langle 21\rangle$ leads to the equivalence of $\langle 22\rangle$ and $\langle 16\rangle$.

The equivalence of $\langle 1\rangle-\langle 5\rangle$ in (b) follows from (3.157). By Lemma 5.2, the matrix equation in $\langle 5\rangle$ holds for all $U$ if and only if

$$
\left[M^{*} M B^{\dagger} A^{\dagger}-M^{*}, M^{*} M B^{\dagger} F_{A}\right]=0 \text { or }\left[\begin{array}{c}
M^{*} M B^{\dagger} A^{\dagger}-M^{*} \\
E_{A}
\end{array}\right]=0
$$

where by $(4.3)$

$$
\begin{aligned}
& r\left[M^{*} M B^{\dagger} A^{\dagger}-M^{*}, M^{*} M B^{\dagger} F_{A}\right]=r\left[\begin{array}{cc}
M^{*} M B^{\dagger} A^{\dagger}-M^{*} & M^{*} M B^{\dagger} \\
0 & A
\end{array}\right]-r(A) \\
& =r\left[\begin{array}{cc}
-B^{*} A^{*} & M^{*} M B^{\dagger} \\
-A A^{\dagger} & A
\end{array}\right]-r(A)=r\left[\begin{array}{cc}
B^{*} A^{*} A & M^{*} M B^{\dagger} \\
A & A
\end{array}\right]-r(A) \\
& =r\left[\begin{array}{cc}
0 & M^{*} M B^{\dagger}-B^{*} A^{*} A \\
A & 0
\end{array}\right]-r(A)=r\left(B B^{\dagger} A^{*} M-A^{*} M\right) \\
& =r\left[B, A^{*} M\right]-r(B) \quad(\text { by }(4.2)),
\end{aligned}
$$

and

$$
\begin{aligned}
r\left[\begin{array}{c}
M^{*} M B^{\dagger} A^{\dagger}-M^{*} \\
E_{A}
\end{array}\right] & =r\left(M^{*} M B^{\dagger} A^{\dagger}-M^{*}\right)+r\left(E_{A}\right) \quad(\text { by Lemma } 4.2(\mathrm{~d})) \\
& =m-r(A)+r\left[B, A^{*} M\right]-r(B) .
\end{aligned}
$$

Combining (9.56) with 9.57$)$ and $(9.58)$ leads to

$$
\begin{aligned}
& {\left[M^{*} M B^{\dagger} F_{A}, M^{*} M B^{\dagger} A^{\dagger}-M^{*}\right]=0} \\
& \Leftrightarrow M^{*} M B^{\dagger} A^{\dagger}-M^{*}=0 \Leftrightarrow \mathscr{R}\left(A^{*} M\right) \subseteq \mathscr{R}(B), \\
& {\left[\begin{array}{c}
M^{*} M B^{\dagger} A^{\dagger}-M^{*} \\
E_{A}
\end{array}\right]=0 \Leftrightarrow \operatorname{both} r(A)=m \text { and } \mathscr{R}\left(A^{*} M\right) \subseteq \mathscr{R}(B) .}
\end{aligned}
$$

Combining 9.59 with 9.60 and (9.56) leads to the equivalence of $\langle 5\rangle$ and $\langle 6\rangle$ in (b). 
The equivalence of $\langle 1\rangle-\langle 5\rangle$ in (c) follows from (3.159). By Lemma 5.2, the matrix equation in $\langle 5\rangle$ holds for all $U$ if and only if

$$
\text { either }\left[M^{*} M B^{\dagger} A^{\dagger}-M^{*}, M^{*} M B^{\dagger} A^{\dagger} A\right]=0 \text { or }\left[\begin{array}{c}
M^{*} M B^{\dagger} A^{\dagger}-M^{*} \\
E_{A}
\end{array}\right]=0
$$

where the rank of the first block matrix in 9.61 is

$$
r\left[M^{*} M B^{\dagger} A^{\dagger}-M^{*}, M^{*} M B^{\dagger} A^{\dagger} A\right]=r\left[-M^{*}, M^{*} M B^{\dagger} A^{\dagger} A\right]=r\left[M^{*}, 0\right]=r(M) \neq 0,
$$

a contradiction to the first equality in (9.61). In this case, combining (9.60) with (9.61) leads to the equivalence of $\langle 5\rangle$ and $\langle 6\rangle$ in $(\mathrm{c})$.

The equivalence of $\langle 1\rangle-\langle 5\rangle$ in (d) follows from (3.160). By Lemma 5.2, the matrix equation in $\langle 5\rangle$ holds for all $U$ if and only if

$$
\text { either }\left[M^{*} M B^{\dagger} A^{\dagger}-M^{*}, M^{*} M B^{\dagger} F_{A}\right]=0 \quad \text { or } \quad\left[\begin{array}{c}
M^{*} M B^{\dagger} A^{\dagger}-M^{*} \\
A A^{\dagger}
\end{array}\right]=0
$$

where the first equality in 9.63 is equivalent to $(9.59)$, and the second equality is a contradiction to $A \neq 0$ Thus $\langle 5\rangle$ and $\langle 6\rangle$ in $(\mathrm{d})$ are equivalent.

The equivalence of $\langle 1\rangle-\langle 5\rangle$ in (e) follows from (3.161). By Lemma 5.2, the matrix equation in $\langle 5\rangle$ holds for all $U$ if and only if

$$
\text { either }\left[M^{*} M B^{\dagger} A^{\dagger}-M^{*}, M^{*} M B^{\dagger}\right]=0 \text { or }\left[\begin{array}{c}
M^{*} M B^{\dagger} A^{\dagger}-M^{*} \\
E_{A}
\end{array}\right]=0,
$$

where the rank of the first block matrix in 9.64 is

$$
r\left[M^{*} M B^{\dagger} A^{\dagger}-M^{*}, M^{*} M B^{\dagger}\right]=r\left[-M^{*}, M^{*} M B^{\dagger}\right]=r\left[M^{*}, 0\right]=r(M) \neq 0,
$$

a contradiction to the first equality in (9.64), while the second equality in (9.64) is equivalent to (9.60). Combining (9.64) with (9.60) and $(9.65)$ leads to the equivalence of $\langle 5\rangle$ and $\langle 6\rangle$ in (e).

The equivalence of $\langle 1\rangle-\langle 5\rangle$ in (f) follows from (3.162). By Lemma 5.2, the matrix equation in $\langle 5\rangle$ holds for all $U$ if and only if $\left[M^{*} M B^{\dagger} A^{\dagger}-M^{*}, M^{*} M B^{\dagger} F_{A}\right]=0$, which is equivalent to (9.59). Thus $\langle 5\rangle$ and $\langle 6\rangle$ in (f) are equivalent.

The equivalence of $\langle 1\rangle-\langle 5\rangle$ in $(\mathrm{g})$ follows from (3.163). By Lemma $5.4(\mathrm{e})$, the matrix equation in $\langle 5\rangle$ holds for all $U_{1}$ and $U_{2}$ if and only if

$$
\left[\begin{array}{cc}
M^{*} M B^{\dagger} A^{\dagger}-M^{*} & M^{*} M B^{\dagger} F_{A} \\
E_{A} & 0
\end{array}\right]=0
$$

where by Lemma $4.2(\mathrm{~d})$ and $(9.57)$

$$
\begin{aligned}
r\left[\begin{array}{cc}
M^{*} M B^{\dagger} A^{\dagger}-M^{*} & M^{*} M B^{\dagger} F_{A} \\
E_{A} & 0
\end{array}\right] & =r\left[M^{*} M B^{\dagger} A^{\dagger}-M^{*}, M^{*} M B^{\dagger} F_{A}\right]+r\left(E_{A}\right) \\
& =m-r(A)+r\left[B, A^{*} M\right]-r(B) .
\end{aligned}
$$

Thus

$$
\left[\begin{array}{cc}
M^{*} M B^{\dagger} A^{\dagger}-M^{*} & M^{*} M B^{\dagger} F_{A} \\
E_{A} & 0
\end{array}\right]=0 \Leftrightarrow r(A)=m \text { and } \mathscr{R}\left(A^{*} M\right) \subseteq \mathscr{R}(B),
$$

establishing the equivalence of $\langle 5\rangle$ and $\langle 6\rangle$ in $(\mathrm{g})$.

The equivalence of $\langle 1\rangle-\langle 5\rangle$ in (h) follows from (3.164). By Lemma $5.3(\mathrm{~b})$, the matrix equation in $\langle 5\rangle$ holds for all $U_{1}$ and $U_{2}$ if and only if $\left[\begin{array}{cc}M^{*} M B^{\dagger} A^{\dagger}-M^{*} & M^{*} M B^{\dagger} F_{A} \\ E_{A} & 0\end{array}\right]=0$, which is equivalent to $\langle 6\rangle$ in $(\mathrm{h})$ by $(9.68)$.

The equivalence of $\langle 1\rangle-\langle 5\rangle$ in (i) follows from (3.165). By Lemma 5.2, the matrix equation in $\langle 5\rangle$ holds for all $V$ if and only if

$$
\left[M^{*} M B^{\dagger} A^{\dagger}-M^{*}, M^{*} M\right]=0 \text { or }\left[\begin{array}{c}
M^{*} M B^{\dagger} A^{\dagger}-M^{*} \\
E_{B} A^{\dagger}
\end{array}\right]=0
$$

where the rank of the first block matrix in 9.69 is

$$
r\left[M^{*} M B^{\dagger} A^{\dagger}-M^{*}, M^{*} M\right]=r\left[0, M^{*} M\right]=r(M) \neq 0,
$$


a contradiction to the first equality in (9.69), and the rank of the second block matrix in (9.69) is

$$
\begin{aligned}
r\left[\begin{array}{c}
M^{*} M B^{\dagger} A^{\dagger}-M^{*} \\
E_{B} A^{\dagger}
\end{array}\right] & =r\left[\begin{array}{cc}
M^{*} M B^{\dagger} A^{\dagger}-M^{*} & 0 \\
A^{\dagger} & B
\end{array}\right]-r(B)=r\left[\begin{array}{cc}
-M^{*} & -M^{*} M \\
A^{\dagger} & B
\end{array}\right]-r(B) \\
& =r\left[\begin{array}{cc}
(A B)^{*} A A^{*} & (A B)^{*} A B \\
A^{*} & B
\end{array}\right]-r(B)=r\left[\begin{array}{cc}
0 & 0 \\
A^{*} & B
\end{array}\right]-r(B) \\
& =r\left[A^{*}, B\right]-r(B) .
\end{aligned}
$$

Thus

$$
\left[\begin{array}{c}
M^{*} M B^{\dagger} A^{\dagger}-M^{*} \\
E_{B} A^{\dagger}
\end{array}\right]=0 \Leftrightarrow r\left[A^{*}, B\right]=r(B) \Leftrightarrow \mathscr{R}\left(A^{*} M\right) \subseteq \mathscr{R}(B) .
$$

Combining (9.69) and $(9.70)$ with $(9.72)$ leads to the equivalence of $\langle 5\rangle$ and $\langle 6\rangle$ in (i).

The equivalence of $\langle 1\rangle-\langle 5\rangle$ in (j) follows from (3.166). By Lemma 5.4(a), the matrix equation in $\langle 5\rangle$ holds for all $U$ and $V$ if and only if

$$
\left[\begin{array}{cc}
M^{*} M B^{\dagger} A^{\dagger}-M^{*} & M^{*} M B^{\dagger} F_{A} \\
E_{B} A^{\dagger} & F_{B} F_{A}
\end{array}\right]=0 \text { or }\left[\begin{array}{c}
M^{*} M B^{\dagger} A^{\dagger}-M^{*} \\
E_{B} A^{\dagger} \\
E_{A}
\end{array}\right]=0
$$

where the ranks of the two block matrices in $(9.73)$ are

$$
\begin{aligned}
r\left[\begin{array}{cc}
M^{*} M B^{\dagger} A^{\dagger}-M^{*} & M^{*} M B^{\dagger} F_{A} \\
E_{B} A^{\dagger} & E_{B} F_{A}
\end{array}\right] & =r\left[\begin{array}{cc}
M^{*} M B^{\dagger} A^{\dagger}-M^{*} & M^{*} A B B^{\dagger}\left(I_{n}-A^{\dagger} A\right) \\
E_{B} A^{\dagger} & E_{B}\left(I_{n}-A^{\dagger} A\right)
\end{array}\right] \\
& =r\left[\begin{array}{cc}
M^{*} A B B^{\dagger} A^{\dagger}-M^{*} & -M^{*} A E_{B} \\
E_{B} A^{\dagger} & E_{B}
\end{array}\right] \\
& =r\left[\begin{array}{cc}
0 & 0 \\
0 & E_{B}
\end{array}\right]=n-r(B) \\
r\left[\begin{array}{c}
M^{*} M B^{\dagger} A^{\dagger}-M^{*} \\
E_{B} A^{\dagger} \\
E_{A}
\end{array}\right] & =r\left[\begin{array}{c}
M^{*} A B B^{\dagger} A^{\dagger}-M^{*} \\
E_{B} A^{\dagger}
\end{array}\right]+r\left(E_{A}\right) \\
& =r\left[\begin{array}{c}
0 \\
E_{B} A^{\dagger}
\end{array}\right]+r\left(E_{A}\right)=r\left[A^{*}, B\right]-r(B)+m-r(A)
\end{aligned}
$$

Combining (9.73) with 9.74 and 9.75 yields

$$
\begin{aligned}
& {\left[\begin{array}{cc}
M^{*} M B^{\dagger} A^{\dagger}-M^{*} & M^{*} M B^{\dagger} F_{A} \\
E_{B} A^{\dagger} & E_{B} F_{A}
\end{array}\right]=0 \Leftrightarrow r(B)=n,} \\
& {\left[\begin{array}{c}
M^{*} M B^{\dagger} A^{\dagger}-M^{*} \\
E_{B} A^{\dagger} \\
E_{A}
\end{array}\right]=0 \Leftrightarrow r(A)=m \text { and } \mathscr{R}\left(A^{*}\right) \subseteq \mathscr{R}(B),}
\end{aligned}
$$

establishing the equivalence of $\langle 5\rangle$ and $\langle 6\rangle$ in $(\mathrm{j})$.

The two groups of equivalent facts in $\langle 1\rangle-\langle 5\rangle$ of $(\mathrm{k})$ and $(\mathrm{m})$ follow from (3.167) and (3.169), respectively. By Lemma 5.4(b), the two matrix equations in $\langle 5\rangle$ of $(\mathrm{k})$ and $(\mathrm{m})$ hold for all $U$ and $V$ if and only if $\left[\begin{array}{c}M^{*} M B^{\dagger} A^{\dagger}-M^{*} \\ E_{B} A^{\dagger} \\ E_{A}\end{array}\right]=0$, which, by 9.77 , is equivalent to $\langle 6\rangle$ in $(\mathrm{k})$ and $(\mathrm{m})$, respectively.

The two groups of equivalent facts in $\langle 1\rangle-\langle 5\rangle$ of $(1)$ and $(n)$ follow from (3.168) and (3.170), respectively. By Lemma 5.4(b), the two matrix equations in $\langle 5\rangle$ holds for all $U$ and $V$ if and only if $\left[\begin{array}{cc}M^{*} M B^{\dagger} A^{\dagger}-M^{*} & M^{*} M B^{\dagger} F_{A} \\ E_{B} A^{\dagger} & F_{B} F_{A}\end{array}\right]=$ 0 , which, by (9.76), is equivalent to $\langle 6\rangle$ in (l) and $(n)$, respectively.

The equivalence of $\langle 1\rangle-\langle 5\rangle$ in (o) follows from (3.171). By Lemma 5.5 (b), the matrix equation in $\langle 5\rangle$ holds for all $U_{1}, U_{2}$, and $V$ if and only if

$$
\left[\begin{array}{cc}
M^{*} M B^{\dagger} A^{\dagger}-M^{*} & M^{*} M B^{\dagger} F_{A} \\
E_{B} A^{\dagger} & E_{B} F_{A} \\
E_{A} & 0
\end{array}\right]=0
$$


where by Lemma $4.2(\mathrm{~d})$ and $(9.74)$

$$
\begin{aligned}
r\left[\begin{array}{cc}
M^{*} M B^{\dagger} A^{\dagger}-M^{*} & M^{*} M B^{\dagger} F_{A} \\
E_{B} A^{\dagger} & E_{B} F_{A} \\
E_{A} & 0
\end{array}\right] & =r\left[\begin{array}{cc}
M^{*} M B^{\dagger} A^{\dagger}-M^{*} & M^{*} M B^{\dagger} F_{A} \\
E_{B} A^{\dagger} & E_{B} F_{A}
\end{array}\right]+r\left(E_{A}\right) \\
& =m-r(A)+n-r(B) .
\end{aligned}
$$

Thus

$$
\left[\begin{array}{cc}
M^{*} M B^{\dagger} A^{\dagger}-M^{*} & M^{*} M B^{\dagger} F_{A} \\
E_{B} A^{\dagger} & E_{B} F_{A} \\
E_{A} & 0
\end{array}\right]=0 \Leftrightarrow r(A)=m \text { and } r(B)=n
$$

establishing the equivalence of $\langle 5\rangle$ and $\langle 6\rangle$ in $(\mathrm{o})$.

The equivalence of $\langle 1\rangle-\langle 5\rangle$ in (p) follows from (3.171). By Lemma 5.5(b), the matrix equation in $\langle 5\rangle$ holds for all $U_{1}, U_{2}$, and $V$ if and only if $(9.79$ holds, which is equivalent to $\langle 6\rangle$ in (p) by 9.80 .

The following theorem can be established by a similar approach, and the details are therefore omitted.

Theorem 9.5. Let $A \in \mathbb{C}^{m \times n}$ and $B \in \mathbb{C}^{n \times p}$ be given, and denote $M=A B$ and $N=\left[A^{*}\right.$, B]. Then the following results hold.

(a) The following 26 statements are equivalent:
$\langle 1\rangle \quad\left\{M^{(1,4)}\right\} \supseteq\left\{B^{(1,3,4)} A^{\dagger}\right\}$.
$\langle 3\rangle \quad\left\{M^{(1,4)}\right\} \supseteq\left\{B^{(1,3,4)} A^{(1,2,4)}\right\}$.
〈5) $\quad M^{\dagger} M=B^{\dagger} A^{\dagger} M$.
$\langle 7\rangle \quad B^{*} A^{\dagger} A B M^{*}=B^{*} B M^{*}$.
$\langle 9\rangle \quad\left(A^{\dagger} A B\right)^{\dagger}=B^{\dagger} A^{\dagger} A$.
$\langle 11\rangle \quad\left(F_{A} B\right)^{\dagger}=B^{\dagger} F_{A}$.
$\langle 13\rangle \quad B M^{\dagger} M=A^{\dagger} M$.
$\langle 15\rangle \quad r\left[B M^{*}, A^{*}\right]=r(A)$.
$\langle 17\rangle \quad \mathscr{R}\left(B M^{*}\right) \subseteq \mathscr{R}\left(A^{*}\right)$.
$\langle 19\rangle \quad \mathscr{R}\left(B M^{*}\right)=\mathscr{R}(B) \cap \mathscr{R}\left(A^{*}\right)$.
$\langle 21\rangle \quad \mathscr{R}\left(A^{\dagger} A B B^{*}\right)=\mathscr{R}\left(B B^{*} A^{\dagger} A\right)$.
$\langle 2\rangle \quad\left\{M^{(1,4)}\right\} \supseteq\left\{B^{(1,3,4)} A^{(1,3,4)}\right\}$.
$\langle 4\rangle \quad\left\{M^{(1,4)}\right\} \supseteq\left\{B^{(1,3,4)} A^{(1,4)}\right\}$.
$\langle 6\rangle \quad B^{\dagger} A^{\dagger} M M^{*}=M^{*}$.
$\langle 8\rangle \quad A^{\dagger} A B M^{*}=B M^{*}$.
$\langle 10\rangle \quad B^{*} B\left(A^{\dagger} A B\right)^{\dagger} A^{*}=M^{*}$.
$\langle 12\rangle \quad\left(F_{A} B\right)^{\dagger} B=B^{\dagger} F_{A} B$.
$\langle 14\rangle \quad A^{\dagger} A B B^{*}=B B^{*} A^{\dagger} A$.
$\langle 16\rangle \quad r\left[B B^{*} F_{A}, F_{A}\right]=r\left(F_{A}\right)$.
$\langle 18\rangle \quad \mathscr{R}\left(B B^{*} F_{A}\right) \subseteq \mathscr{N}(A)$.
$\langle 23\rangle \quad B^{\dagger} A^{\dagger} M$ is an orthogonal projector, i.e., $\left(B^{\dagger} A^{\dagger} M\right)^{2}=B^{\dagger} A^{\dagger} M=\left(B^{\dagger} A^{\dagger} M\right)^{*}$.
$\langle 24\rangle \quad B^{\dagger} F_{A} B$ is an orthogonal projector, i.e., $\left(B^{\dagger} F_{A} B\right)^{2}=B^{\dagger} F_{A} B=\left(B^{\dagger} F_{A} B\right)^{*}$.
$\langle 25\rangle$ Both $M B^{\dagger} A^{\dagger} M=M$ and $\left(B^{\dagger} A^{\dagger} M\right)^{*}=B^{\dagger} A^{\dagger} M$.
$\langle 26\rangle \quad$ Both $r(N)=r(A)+r(B)-r(M)$ and $r\left[B^{*} B M^{*}, M^{*}\right]=r(M)$.

(b) The following 6 statements are equivalent:
$\langle 1\rangle \quad\left\{M^{(1,4)}\right\} \supseteq\left\{B^{(1,3,4)} A^{\dagger}\right\}$.
$\langle 3\rangle \quad\left\{M^{(1,4)}\right\} \supseteq\left\{B^{(1,3,4)} A^{(1,2,4)}\right\}$.
$\langle 5\rangle \quad F_{B} V E_{B} A^{\dagger} M M^{*} \equiv M^{*}-B^{\dagger} A^{\dagger} M M^{*}$ for all $V$.
$\langle 2\rangle \quad\left\{M^{(1,4)}\right\} \supseteq\left\{B^{(1,3,4)} A^{(1,3,4)}\right\}$.
$\langle 6\rangle \quad \mathscr{R}\left(B M^{*}\right) \subseteq \mathscr{R}\left(A^{*}\right)$.

(c) The following 6 statements are equivalent:
$\langle 1\rangle \quad\left\{M^{(1,4)}\right\} \supseteq\left\{B^{(1,2,4)} A^{\dagger}\right\}$.
$\langle 3\rangle \quad\left\{M^{(1,4)}\right\} \supseteq\left\{B^{(1,2,4)} A^{(1,2,4)}\right\}$.
$\langle 2\rangle \quad\left\{M^{(1,4)}\right\} \supseteq\left\{B^{(1,2,4)} A^{(1,3,4)}\right\}$.
〈5) $\quad B^{\dagger} B V E_{B} A^{\dagger} M M^{*} \equiv M^{*}-B^{\dagger} A^{\dagger} M M^{*}$ for all $V$.
$\langle 6\rangle \quad \mathscr{R}\left(B M^{*}\right) \subseteq \mathscr{R}\left(A^{*}\right)$.

(d) The following 6 statements are equivalent:
$\langle 1\rangle \quad\left\{M^{(1,4)}\right\} \supseteq\left\{B^{(1,2,3)} A^{\dagger}\right\}$.
$\langle 3\rangle \quad\left\{M^{(1,4)}\right\} \supseteq\left\{B^{(1,2,3)} A^{(1,2,4)}\right\}$.
$\langle 2\rangle \quad\left\{M^{(1,4)}\right\} \supseteq\left\{B^{(1,2,3)} A^{(1,3,4)}\right\}$.
$\langle 5\rangle \quad F_{B} V B B^{\dagger} A^{\dagger} M M^{*} \equiv M^{*}-B^{\dagger} A^{\dagger} M M^{*}$ for all $V$.
$\langle 6\rangle$ Both $r(B)=p$ and $\mathscr{R}\left(B M^{*}\right) \subseteq \mathscr{R}\left(A^{*}\right)$. 
(e) The following 6 statements are equivalent:
$\langle 1\rangle \quad\left\{M^{(1,4)}\right\} \supseteq\left\{B^{(1,4)} A^{\dagger}\right\}$.
$\langle 2\rangle \quad\left\{M^{(1,4)}\right\} \supseteq\left\{B^{(1,4)} A^{(1,3,4)}\right\}$.
$\langle 3\rangle \quad\left\{M^{(1,4)}\right\} \supseteq\left\{B^{(1,4)} A^{(1,2,4)}\right\}$.
〈4) $\left\{M^{(1,4)}\right\} \supseteq\left\{B^{(1,4)} A^{(1,4)}\right\}$.
<5) $V E_{B} A^{\dagger} M M^{*} \equiv M^{*}-B^{\dagger} A^{\dagger} M M^{*}$ for all $V$.
$\langle 6\rangle \quad \mathscr{R}\left(B M^{*}\right) \subseteq \mathscr{R}\left(A^{*}\right)$.

(f) The following 6 statements are equivalent:
〈1) $\quad\left\{M^{(1,4)}\right\} \supseteq\left\{B^{(1,3)} A^{\dagger}\right\}$.
$\langle 2\rangle \quad\left\{M^{(1,4)}\right\} \supseteq\left\{B^{(1,3)} A^{(1,3,4)}\right\}$.
$\langle 3\rangle \quad\left\{M^{(1,4)}\right\} \supseteq\left\{B^{(1,3)} A^{(1,2,4)}\right\}$.
〈4〉 $\left\{M^{(1,4)}\right\} \supseteq\left\{B^{(1,3)} A^{(1,4)}\right\}$.
$\langle 5\rangle \quad F_{B} V A^{\dagger} M M^{*} \equiv M^{*}-B^{\dagger} A^{\dagger} M M^{*}$ for all $V$.
$\langle 6\rangle$ Both $r(B)=p$ and $\mathscr{R}\left(B M^{*}\right) \subseteq \mathscr{R}\left(A^{*}\right)$.

(g) The following 6 statements are equivalent:
〈1) $\quad\left\{M^{(1,4)}\right\} \supseteq\left\{B^{(1,2)} A^{\dagger}\right\}$.
$\langle 2\rangle \quad\left\{M^{(1,4)}\right\} \supseteq\left\{B^{(1,2)} A^{(1,3,4)}\right\}$.
$\langle 3\rangle \quad\left\{M^{(1,4)}\right\} \supseteq\left\{B^{(1,2)} A^{(1,2,4)}\right\}$.
$\langle 4\rangle \quad\left\{M^{(1,4)}\right\} \supseteq\left\{B^{(1,2)} A^{(1,4)}\right\}$.
$\langle 5\rangle \quad\left(B^{\dagger}+F_{B} V_{1}\right)\left(B B^{\dagger} A^{\dagger} M M^{*}+B V_{2} E_{B} A^{\dagger} M M^{*}\right) \equiv M^{*}$ for all $V_{1}$ and $V_{2}$.
$\langle 6\rangle \quad$ Both $r(B)=p$ and $\mathscr{R}\left(B M^{*}\right) \subseteq \mathscr{R}\left(A^{*}\right)$.

(h) The following 6 statements are equivalent:
$\langle 1\rangle \quad\left\{M^{(1,4)}\right\} \supseteq\left\{B^{(1)} A^{\dagger}\right\}$.
$\langle 3\rangle \quad\left\{M^{(1,4)}\right\} \supseteq\left\{B^{(1)} A^{(1,2,4)}\right\}$.
$\langle 2\rangle \quad\left\{M^{(1,4)}\right\} \supseteq\left\{B^{(1)} A^{(1,3,4)}\right\}$.
<4 $\left\{M^{(1,4)}\right\} \supseteq\left\{B^{(1)} A^{(1,4)}\right\}$.
$\langle 5\rangle \quad F_{B} V_{1} A^{\dagger} M M^{*}+V_{2} E_{B} A^{\dagger} M M^{*} \equiv M^{*}-B^{\dagger} A^{\dagger} M M^{*}$ for all $V_{1}$ and $V_{2}$.
$\langle 6\rangle$ Both $r(B)=p$ and $\mathscr{R}\left(B M^{*}\right) \subseteq \mathscr{R}\left(A^{*}\right)$.

(i) The following 6 statements are equivalent:
$\langle 1\rangle \quad\left\{M^{(1,4)}\right\} \supseteq\left\{B^{\dagger} A^{(1,2,3)}\right\}$.
$\langle 2\rangle \quad\left\{M^{(1,4)}\right\} \supseteq\left\{B^{\dagger} A^{(1,3)}\right\}$.
$\langle 3\rangle \quad\left\{M^{(1,4)}\right\} \supseteq\left\{B^{\dagger} A^{(1,2)}\right\}$.
$\langle 4\rangle \quad\left\{M^{(1,4)}\right\} \supseteq\left\{B^{\dagger} A^{(1)}\right\}$.
〈5〉 $\quad B^{\dagger} F_{A} U M M^{*} \equiv M^{*}-B^{\dagger} A^{\dagger} M M^{*}$ for all $U$.
$\langle 6\rangle \quad \mathscr{R}\left(A^{*}\right) \supseteq \mathscr{R}(B)$.

(j) The following 6 statements are equivalent:
$\langle 1\rangle \quad\left\{M^{(1,4)}\right\} \supseteq\left\{B^{(1,3,4)} A^{(1,2,3)}\right\}$.
$\langle 2\rangle \quad\left\{M^{(1,4)}\right\} \supseteq\left\{B^{(1,3,4)} A^{(1,3)}\right\}$.
$\langle 3\rangle \quad\left\{M^{(1,4)}\right\} \supseteq\left\{B^{(1,3,4)} A^{(1,2)}\right\}$.
$\langle 4\rangle \quad\left\{M^{(1,4)}\right\} \supseteq\left\{B^{(1,3,4)} A^{(1)}\right\}$.
$\langle 5\rangle \quad\left(B^{\dagger}+F_{B} V E_{B}\right)\left(A^{\dagger} M M^{*}+F_{A} U M M^{*}\right) \equiv M^{*}$ for all $U$ and $V$.
$\langle 6\rangle \quad$ Either $r(A)=n$ or $\left\{r(B)=p\right.$ and $\left.\mathscr{R}\left(A^{*}\right) \supseteq \mathscr{R}(B)\right\}$.

(k) The following 6 statements are equivalent:
$\langle 1\rangle \quad\left\{M^{(1,4)}\right\} \supseteq\left\{B^{(1,2,3)} A^{(1,2,3)}\right\}$.
$\langle 2\rangle \quad\left\{M^{(1,4)}\right\} \supseteq\left\{B^{(1,2,3)} A^{(1,3)}\right\}$.
$\langle 3\rangle \quad\left\{M^{(1,4)}\right\} \supseteq\left\{B^{(1,2,3)} A^{(1,2)}\right\}$.
$\langle 4\rangle \quad\left\{M^{(1,4)}\right\} \supseteq\left\{B^{(1,2,3)} A^{(1)}\right\}$.
$\langle 5\rangle \quad\left(B^{\dagger}+F_{B} V B B^{\dagger}\right)\left(A^{\dagger} M M^{*}+F_{A} U M M^{*}\right) \equiv M^{*}$ for all $U$ and $V$.
$\langle 6\rangle \quad$ Both $r(B)=p$ and $\mathscr{R}\left(A^{*}\right) \supseteq \mathscr{R}(B)$.

(1) The following 6 statements are equivalent:
$\langle 1\rangle \quad\left\{M^{(1,4)}\right\} \supseteq\left\{B^{(1,2,4)} A^{(1,2,3)}\right\}$.
$\langle 2\rangle \quad\left\{M^{(1,4)}\right\} \supseteq\left\{B^{(1,2,4)} A^{(1,3)}\right\}$.
$\langle 3\rangle \quad\left\{M^{(1,4)}\right\} \supseteq\left\{B^{(1,2,4)} A^{(1,2)}\right\}$.
$\langle 4\rangle \quad\left\{M^{(1,4)}\right\} \supseteq\left\{B^{(1,2,4)} A^{(1)}\right\}$.
$\langle 5\rangle \quad\left(B^{\dagger}+B^{\dagger} B V E_{B}\right)\left(A^{\dagger} M M^{*}+F_{A} U M M^{*}\right) \equiv M^{*}$ for all $U$ and $V$.
$\langle 6\rangle \quad r(A)=n$.

(m) The following 6 statements are equivalent:
$\langle 1\rangle \quad\left\{M^{(1,4)}\right\} \supseteq\left\{B^{(1,3)} A^{(1,2,3)}\right\}$.
$\langle 2\rangle \quad\left\{M^{(1,4)}\right\} \supseteq\left\{B^{(1,3)} A^{(1,3)}\right\}$.
$\langle 3\rangle \quad\left\{M^{(1,4)}\right\} \supseteq\left\{B^{(1,3)} A^{(1,2)}\right\}$.
$\langle 4\rangle \quad\left\{M^{(1,4)}\right\} \supseteq\left\{B^{(1,3)} A^{(1)}\right\}$.
$\langle 5\rangle \quad\left(B^{\dagger}+F_{B} V\right)\left(A^{\dagger} M M^{*}+F_{A} U M M^{*}\right) \equiv M^{*}$ for all $U$ and $V$.
$\langle 6\rangle \quad$ Both $r(B)=p$ and $\mathscr{R}\left(A^{*}\right) \supseteq \mathscr{R}(B)$. 
(n) The following 6 statements are equivalent:
$\langle 1\rangle \quad\left\{M^{(1,4)}\right\} \supseteq\left\{B^{(1,4)} A^{(1,2,3)}\right\}$.
$\langle 2\rangle \quad\left\{M^{(1,4)}\right\} \supseteq\left\{B^{(1,4)} A^{(1,3)}\right\}$.
$\langle 3\rangle \quad\left\{M^{(1,4)}\right\} \supseteq\left\{B^{(1,4)} A^{(1,2)}\right\}$.
$\langle 4\rangle \quad\left\{M^{(1,4)}\right\} \supseteq\left\{B^{(1,4)} A^{(1)}\right\}$.
$\langle 5\rangle \quad\left(B^{\dagger}+V E_{B}\right)\left(A^{\dagger} M M^{*}+F_{A} U M M^{*}\right) \equiv M^{*}$ for all $U$ and $V$.
$\langle 6\rangle \quad r(A)=n$.

(o) The following 6 statements are equivalent:
$\langle 1\rangle \quad\left\{M^{(1,4)}\right\} \supseteq\left\{B^{(1,2)} A^{(1,2,3)}\right\}$.
$\langle 3\rangle \quad\left\{M^{(1,4)}\right\} \supseteq\left\{B^{(1,2)} A^{(1,2)}\right\}$.
$\langle 2\rangle \quad\left\{M^{(1,4)}\right\} \supseteq\left\{B^{(1,2)} A^{(1,3)}\right\}$.
$\langle 5\rangle \quad\left(B^{\dagger}+F_{B} V_{1}\right) B\left(B^{\dagger}+V_{2} E_{B}\right)\left(A^{\dagger} M M^{*}+F_{A} U M M^{*}\right) \equiv M^{*}$ for all $U, V_{1}$, and $V_{2}$.
$\langle 6\rangle \quad$ Both $r(A)=n$ and $r(B)=p$.

(p) The following 6 statements are equivalent:
$\langle 1\rangle \quad\left\{M^{(1,4)}\right\} \supseteq\left\{B^{(1)} A^{(1,2,3)}\right\}$.
$\langle 3\rangle \quad\left\{M^{(1,4)}\right\} \supseteq\left\{B^{(1)} A^{(1,2)}\right\}$.
$\langle 2\rangle \quad\left\{M^{(1,4)}\right\} \supseteq\left\{B^{(1)} A^{(1,3)}\right\}$.
$\langle 5\rangle \quad\left(B^{\dagger}+F_{B} V_{1}+V_{2} E_{B}\right)\left(A^{\dagger} M M^{*}+F_{A} U M M^{*}\right) \equiv M^{*}$ for all $U, V_{1}$, and $V_{2}$.
$\langle 6\rangle$ Both $r(A)=n$ and $r(B)=p$.

\section{Set inclusions for $\{1,2,3\}-,\{1,2,4\}-$, and $\{1,3,4\}$-generalized invers- es of $A B$}

Applying Lemma 3.2 to Theorems 9.3 9.5, we obtain the following theorems, the proofs underlying which are omitted.

Theorem 10.1. Let $A \in \mathbb{C}^{m \times n}$ and $B \in \mathbb{C}^{n \times p}$ be given, and denote $M=A B$ and $t=m+p+r(M)-r(A)-r(B)$. Then the following results hold.

(a) The following 3 statements are equivalent:

$$
\langle 1\rangle \quad\left\{M^{(1,2,3)}\right\} \ni B^{\dagger} A^{\dagger} . \quad\langle 2\rangle \quad\left\{M^{(1,2,3)}\right\} \supseteq\left\{B^{(1,2,3)} A^{\dagger}\right\} . \quad\langle 3\rangle \quad \mathscr{R}\left(A^{*} M\right) \subseteq \mathscr{R}(B) .
$$

(b) The following 3 statements are equivalent:
$\langle 1\rangle \quad\left\{M^{(1,2,3)}\right\} \supseteq\left\{B^{\dagger} A^{(1,2,3)}\right\}$.
$\langle 3\rangle \quad$ Either $\mathscr{R}\left(\bar{A}^{*}\right) \subseteq \mathscr{R}(B)$ or $\mathscr{R}\left(A^{*} M\right)=\mathscr{R}(B)$.
$\langle 2\rangle \quad\left\{M^{(1,2,3)}\right\} \supseteq\left\{B^{(1,2,3)} A^{(1,2,3)}\right\}$.

(c) The following 5 statements are equivalent:
$\langle 1\rangle \quad\left\{M^{(1,2,3)}\right\} \supseteq\left\{B^{\dagger} A^{(1,2,4)}\right\}$.
$\langle 2\rangle \quad\left\{M^{(1,2,3)}\right\} \supseteq\left\{B^{(1,2,3)} A^{(1,2,4)}\right\}$.
$\langle 3\rangle \quad\left\{M^{(1,2,3)}\right\} \supseteq\left\{B^{\dagger} A^{(1,4)}\right\}$.
$\langle 4\rangle \quad\left\{M^{(1,2,3)}\right\} \supseteq\left\{B^{(1,2,3)} A^{(1,4)}\right\}$.
$\langle 5\rangle \quad$ Both $r(A)=m$ and $\mathscr{R}\left(A^{*} M\right) \subseteq \mathscr{R}(B)$.

(d) The following 5 statements are equivalent:
$\langle 1\rangle \quad\left\{M^{(1,2,3)}\right\} \supseteq\left\{B^{\dagger} A^{(1,2)}\right\}$.
$\langle 3\rangle \quad\left\{M^{(1,2,3)}\right\} \supseteq\left\{B^{(1,3,4)} A^{(1,2)}\right\}$.
(5) Either $\left\{r(A)=m\right.$ and $\left.\mathscr{R}\left(A^{*}\right) \subseteq \mathscr{R}(B)\right\}$ or
$\langle 2\rangle \quad\left\{M^{(1,2,3)}\right\} \supseteq\left\{B^{(1,2,3)} A^{(1,2)}\right\}$.
〈4) $\left\{M^{(1,2,3)}\right\} \supseteq\left\{B^{(1,3)} A^{(1,2)}\right\}$.
$\left\{r(A)=m, r(B)=p\right.$, and $\left.\mathscr{R}\left(A^{*} M\right)=\mathscr{R}(B)\right\}$.

(e) The following 5 statements are equivalent:
〈1) $\left\{M^{(1,2,3)}\right\} \supseteq\left\{B^{(1,2,4)} A^{\dagger}\right\}$.
$\langle 2\rangle \quad\left\{M^{(1,2,3)}\right\} \supseteq\left\{B^{(1,4)} A^{\dagger}\right\}$.
$\langle 3\rangle \quad\left\{M^{(1,2,3)}\right\} \supseteq\left\{B^{(1,2)} A^{\dagger}\right\}$.
$\langle 4\rangle \quad\left\{M^{(1,2,3)}\right\} \supseteq\left\{B^{(1)} A^{\dagger}\right\}$.
$\langle 5\rangle \quad \mathscr{R}\left(A^{*}\right) \subseteq \mathscr{R}(B)$.

(f) The following 9 statements are equivalent:
$\langle 1\rangle \quad\left\{M^{(1,2,3)}\right\} \supseteq\left\{B^{(1,2,4)} A^{(1,2,4)}\right\}$.
$\langle 2\rangle \quad\left\{M^{(1,2,3)}\right\} \supseteq\left\{B^{(1,2)} A^{(1,2,4)}\right\}$.
$\langle 3\rangle \quad\left\{M^{(1,2,3)}\right\} \supseteq\left\{B^{(1,4)} A^{(1,2,4)}\right\}$.
$\langle 4\rangle \quad\left\{M^{(1,2,3)}\right\} \supseteq\left\{B^{(1)} A^{(1,2,4)}\right\}$.
$\langle 5\rangle \quad\left\{M^{(1,2,3)}\right\} \supseteq\left\{B^{(1,2,4)} A^{(1,4)}\right\}$.
$\langle 6\rangle \quad\left\{M^{(1,2,3)}\right\} \supseteq\left\{B^{(1,2)} A^{(1,4)}\right\}$.
$\langle 7\rangle \quad\left\{M^{(1,2,3)}\right\} \supseteq\left\{B^{(1,4)} A^{(1,4)}\right\}$.
$\langle 8\rangle \quad\left\{M^{(1,2,3)}\right\} \supseteq\left\{B^{(1)} A^{(1,4)}\right\}$.

$\langle 9\rangle \quad$ Both $r(A)=m$ and $\mathscr{R}\left(A^{*}\right) \subseteq \mathscr{R}(B)$. 
(g) The following 3 statements are equivalent:
$\langle 1\rangle \quad\left\{M^{(1,2,3)}\right\} \supseteq\left\{B^{(1,3,4)} A^{(1,2,3)}\right\}$.
$\langle 2\rangle \quad\left\{M^{(1,2,3)}\right\} \supseteq\left\{B^{(1,3)} A^{(1,2,3)}\right\}$.
$\langle 3\rangle \quad$ Either $\mathscr{R}\left(A^{*}\right) \subseteq \mathscr{R}(B)$ or $\left\{r(B)=p\right.$ and $\left.\mathscr{R}\left(A^{*} M\right)=\mathscr{R}(B)\right\}$.

(h) The following 3 statements are equivalent:
〈1) $\quad\left\{M^{(1,2,3)}\right\} \supseteq\left\{B^{\dagger} A^{(1,3)}\right\}$.
$\langle 2\rangle \quad\left\{M^{(1,2,3)}\right\} \supseteq\left\{B^{(1,2,3)} A^{(1,3)}\right\}$.
$\langle 3\rangle \quad$ Either $\mathscr{R}\left(A^{*} M\right)=\mathscr{R}(B)$ or $\left\{r(A)=m\right.$ and $\left.\mathscr{R}\left(A^{*}\right) \subseteq \mathscr{R}(B)\right\}$.

(i) The following 3 statements are equivalent:
$\langle 1\rangle \quad\left\{M^{(1,2,3)}\right\} \supseteq\left\{B^{\dagger} A^{(1)}\right\}$.
$\langle 2\rangle \quad\left\{M^{(1,2,3)}\right\} \supseteq\left\{B^{(1,2,3)} A^{(1)}\right\}$.
$\langle 3\rangle$ Either $\left\{r(A)=m\right.$ and $\left.\mathscr{R}\left(A^{*} M\right)=\mathscr{R}(B)\right\}$ or $\left\{r(A)=m\right.$ and $\left.\mathscr{R}\left(A^{*}\right) \subseteq \mathscr{R}(B)\right\}$.

(j) The following 5 statements are equivalent:
$\langle 1\rangle \quad\left\{M^{(1,2,3)}\right\} \supseteq\left\{B^{(1,2,4)} A^{(1,3,4)}\right\}$.
$\langle 3\rangle \quad\left\{M^{(1,2,3)}\right\} \supseteq\left\{B^{(1,4)} A^{(1,3,4)}\right\}$.
$\langle 2\rangle \quad\left\{M^{(1,2,3)}\right\} \supseteq\left\{B^{(1,2)} A^{(1,3,4)}\right\}$.
$\langle 5\rangle$ Either $r(M)=n$ or $\left\{r(A)=m\right.$ and $\left.\mathscr{R}\left(A^{*}\right) \subseteq \mathscr{R}(B)\right\}$.

(k) The following 3 statements are equivalent:
$\langle 1\rangle \quad\left\{M^{(1,2,3)}\right\} \supseteq\left\{B^{(1,3,4)} A^{\dagger}\right\}$.
$\langle 2\rangle \quad\left\{M^{(1,2,3)}\right\} \supseteq\left\{B^{(1,3)} A^{\dagger}\right\}$.
$\langle 3\rangle$ Either $\mathscr{R}\left(A^{*}\right) \subseteq \mathscr{R}(B)$ or $\left\{\mathscr{R}\left(A^{*} M\right) \subseteq \mathscr{R}(B)\right.$ and $\left.r(B)=p\right\}$.

(1) The following 3 statements are equivalent:
$\langle 1\rangle \quad\left\{M^{(1,2,3)}\right\} \supseteq\left\{B^{(1,3,4)} A^{(1,2,4)}\right\}$.
$\langle 3\rangle \quad$ Either $\left\{r(\bar{A})=m\right.$ and $\left.\mathscr{R}\left(A^{*}\right) \subseteq \mathscr{R}(B)\right\}$
$\langle 2\rangle \quad\left\{M^{(1,2,3)}\right\} \supseteq\left\{B^{(1,3)} A^{(1,2,4)}\right\}$. or $\left\{r(A)=m, r(B)=p\right.$, and $\left.\mathscr{R}\left(A^{*} M\right) \subseteq \mathscr{R}(B)\right\}$.

(m) The following 3 statements are equivalent:
$\langle 1\rangle \quad\left\{M^{(1,2,3)}\right\} \supseteq\left\{B^{\dagger} A^{(1,3,4)}\right\}$.
$\langle 2\rangle \quad\left\{M^{(1,2,3)}\right\} \supseteq\left\{B^{(1,2,3)} A^{(1,3,4)}\right\}$.
$\langle 3\rangle \quad$ Either $\mathscr{R}\left(A^{*} M\right)=\mathscr{R}(B)$, or $\left\{r(A)=m\right.$ and $\left.\mathscr{R}\left(A^{*} M\right) \subseteq \mathscr{R}(B)\right\}$.

(n) The following 3 statements are equivalent:
〈1) $\quad\left\{M^{(1,2,3)}\right\} \supseteq\left\{B^{(1,3,4)} A^{(1,3)}\right\}$.
$\langle 2\rangle \quad\left\{M^{(1,2,3)}\right\} \supseteq\left\{B^{(1,3)} A^{(1,3)}\right\}$.
$\langle 3\rangle \quad$ Both $r(M)=\min \{m, n, p\}$ and $\mathscr{R}\left(A^{*} M\right) \subseteq \mathscr{R}(B)$.

(o) The following 3 statements are equivalent:
$\langle 1\rangle \quad\left\{M^{(1,2,3)}\right\} \supseteq\left\{B^{(1,3,4)} A^{(1)}\right\}$.
$\langle 2\rangle \quad\left\{M^{(1,2,3)}\right\} \supseteq\left\{B^{(1,3)} A^{(1)}\right\}$.
$\langle 3\rangle \quad r(A)=m, r(M)=\min \{m, n, p\}$, and $\mathscr{R}\left(A^{*} M\right) \subseteq \mathscr{R}(B)$.

(p) The following 3 statements are equivalent:
$\langle 1\rangle \quad\left\{M^{(1,2,3)}\right\} \supseteq\left\{B^{(1,3,4)} A^{(1,3,4)}\right\}$.
$\langle 2\rangle \quad\left\{M^{(1,2,3)}\right\} \supseteq\left\{B^{(1,3)} A^{(1,3,4)}\right\}$.
$\langle 3\rangle \quad$ Both $\mathscr{R}\left(A^{*} M\right) \subseteq \mathscr{R}(B)$ and $r(M)=\min \{m, n, p, t\}$.

(q) The following 3 statements are equivalent:
$\langle 1\rangle \quad\left\{M^{(1,2,3)}\right\} \supseteq\left\{B^{(1,3,4)} A^{(1,4)}\right\}$.
$\langle 2\rangle \quad\left\{M^{(1,2,3)}\right\} \supseteq\left\{B^{(1,3)} A^{(1,4)}\right\}$.
$\langle 3\rangle \quad r(A)=m, \mathscr{R}\left(A^{*} M\right) \subseteq \mathscr{R}(B)$, and $r(M)=\min \{m, p, t\}$.

(r) The following 9 statements are equivalent:
$\langle 1\rangle \quad\left\{M^{(1,2,3)}\right\} \supseteq\left\{B^{(1,2,4)} A^{(1,2,3)}\right\}$.
$\langle 3\rangle \quad\left\{M^{(1,2,3)}\right\} \supseteq\left\{B^{(1,2,4)} A^{(1,3)}\right\}$.
$\langle 2\rangle \quad\left\{M^{(1,2,3)}\right\} \supseteq\left\{B^{(1,2)} A^{(1,2,3)}\right\}$.
$\langle 5\rangle \quad\left\{M^{(1,2,3)}\right\} \supseteq\left\{B^{(1,4)} A^{(1,2,3)}\right\}$.
$\langle 7\rangle \quad\left\{M^{(1,2,3)}\right\} \supseteq\left\{B^{(1,4)} A^{(1,3)}\right\}$.
$\langle 4\rangle \quad\left\{M^{(1,2,3)}\right\} \supseteq\left\{B^{(1,2)} A^{(1,3)}\right\}$.
$\langle 9\rangle \quad r(M)=n$.
$\langle 6\rangle \quad\left\{M^{(1,2,3)}\right\} \supseteq\left\{B^{(1)} A^{(1,2,3)}\right\}$.
$\langle 8\rangle \quad\left\{M^{(1,2,3)}\right\} \supseteq\left\{B^{(1)} A^{(1,3)}\right\}$. 
(s) The following 9 statements are equivalent:
$\langle 1\rangle \quad\left\{M^{(1,2,3)}\right\} \supseteq\left\{B^{(1,2,4)} A^{(1,2)}\right\}$.
$\langle 3\rangle \quad\left\{M^{(1,2,3)}\right\} \supseteq\left\{B^{(1,2,4)} A^{(1)}\right\}$.
$\langle 2\rangle \quad\left\{M^{(1,2,3)}\right\} \supseteq\left\{B^{(1,2)} A^{(1,2)}\right\}$.
$\langle 5\rangle \quad\left\{M^{(1,2,3)}\right\} \supseteq\left\{B^{(1,4)} A^{(1,2)}\right\}$.
$\langle 4\rangle \quad\left\{M^{(1,2,3)}\right\} \supseteq\left\{B^{(1,2)} A^{(1)}\right\}$.
$\langle 7\rangle \quad\left\{M^{(1,2,3)}\right\} \supseteq\left\{B^{(1,4)} A^{(1)}\right\}$.
$\langle 6\rangle \quad\left\{M^{(1,2,3)}\right\} \supseteq\left\{B^{(1)} A^{(1,2)}\right\}$.
$\langle 9\rangle \quad r(M)=m=n$.
$\langle 8\rangle \quad\left\{M^{(1,2,3)}\right\} \supseteq\left\{B^{(1)} A^{(1)}\right\}$.

Theorem 10.2. Let $A \in \mathbb{C}^{m \times n}$ and $B \in \mathbb{C}^{n \times p}$ be given, and denote $M=A B$ and $t=m+p+r(M)-r(A)-r(B)$. Then the following results hold.

(a) The following 3 statements are equivalent:
$\langle 1\rangle \quad\left\{M^{(1,2,4)}\right\} \ni B^{\dagger} A^{\dagger}$
$\langle 2\rangle \quad\left\{M^{(1,2,4)}\right\} \supseteq\left\{B^{\dagger} A^{(1,2,4)}\right\}$.
$\langle 3\rangle \mathscr{R}\left(B M^{*}\right) \subseteq \mathscr{R}\left(A^{*}\right)$.

(b) The following 3 statements are equivalent:
$\langle 1\rangle \quad\left\{M^{(1,2,4)}\right\} \supseteq\left\{B^{(1,2,4)} A^{\dagger}\right\}$.
$\langle 3\rangle \quad$ Either $\mathscr{R}\left(A^{*}\right) \supseteq \mathscr{R}(B)$ or $\mathscr{R}\left(B M^{*}\right)=\mathscr{R}\left(A^{*}\right)$.
$\langle 2\rangle \quad\left\{M^{(1,2,4)}\right\} \supseteq\left\{B^{(1,2,4)} A^{(1,2,4)}\right\}$.

(c) The following 5 statements are equivalent:
$\langle 1\rangle \quad\left\{M^{(1,2,4)}\right\} \supseteq\left\{B^{(1,2,3)} A^{\dagger}\right\}$.
$\langle 3\rangle \quad\left\{M^{(1,2,4)}\right\} \supseteq\left\{B^{(1,4)} A^{\dagger}\right\}$.
$\langle 2\rangle \quad\left\{M^{(1,2,4)}\right\} \supseteq\left\{B^{(1,2,3)} A^{(1,2,4)}\right\}$.
$\langle 4\rangle \quad\left\{M^{(1,2,4)}\right\} \supseteq\left\{B^{(1,3)} A^{(1,2,4)}\right\}$.

$\langle 5\rangle \quad$ Both $r(B)=p$ and $\mathscr{R}\left(B M^{*}\right) \subseteq \mathscr{R}\left(A^{*}\right)$.

(d) The following 5 statements are equivalent:
$\langle 1\rangle \quad\left\{M^{(1,2,4)}\right\} \supseteq\left\{B^{(1,2)} A^{\dagger}\right\}$.
$\begin{array}{ll}\langle 3\rangle & \left\{M^{(1,2,4)}\right\} \supseteq\left\{B^{(1,2)} A^{(1,3,4)}\right\} . \quad\langle 4\rangle \\ \langle 5\rangle & \text { Either }\left\{r(B)=p \text { and } \mathscr{R}\left(A^{*}\right) \supseteq \mathscr{R}(B)\right\} \\ & \text { or }\left\{r(A)=m, r(B)=p, \text { and } \mathscr{R}\left(B M^{*}\right)\right.\end{array}$
$\langle 2\rangle \quad\left\{M^{(1,2,4)}\right\} \supseteq\left\{B^{(1,2)} A^{(1,2,4)}\right\}$.
or $\left\{r(A)=m, r(B)=p\right.$, and $\left.\mathscr{R}\left(B M^{*}\right)=\mathscr{R}\left(A^{*}\right)\right\}$.

(e) The following 5 statements are equivalent:
$\langle 1\rangle \quad\left\{M^{(1,2,4)}\right\} \supseteq\left\{B^{\dagger} A^{(1,2,3)}\right\}$.
$\langle 3\rangle \quad\left\{M^{(1,2,4)}\right\} \supseteq\left\{B^{\dagger} A^{(1,2)}\right\}$.
$\langle 2\rangle \quad\left\{M^{(1,2,4)}\right\} \supseteq\left\{B^{\dagger} A^{(1,3)}\right\}$.
$\langle 5\rangle \quad \mathscr{R}\left(A^{*}\right) \supseteq \mathscr{R}(B)$.
$\langle 4\rangle \quad\left\{M^{(1,2,4)}\right\} \supseteq\left\{B^{\dagger} A^{(1)}\right\}$.

(f) The following 9 statements are equivalent:
$\langle 1\rangle \quad\left\{M^{(1,2,4)}\right\} \supseteq\left\{B^{(1,2,4)} A^{(1,2,3)}\right\}$.
$\langle 3\rangle \quad\left\{M^{(1,2,4)}\right\} \supseteq\left\{B^{(1,2,3)} A^{(1,3)}\right\}$.
$\langle 2\rangle \quad\left\{M^{(1,2,4)}\right\} \supseteq\left\{B^{(1,2,3)} A^{(1,2)}\right\}$.
$\langle 5\rangle \quad\left\{M^{(1,2,4)}\right\} \supseteq\left\{B^{(1,3)} A^{(1,2,3)}\right\}$.
$\langle 4\rangle \quad\left\{M^{(1,2,4)}\right\} \supseteq\left\{B^{(1,2,3)} A^{(1)}\right\}$.
$\langle 7\rangle \quad\left\{M^{(1,2,4)}\right\} \supseteq\left\{B^{(1,3)} A^{(1,3)}\right\}$.
$\langle 6\rangle \quad\left\{M^{(1,2,4)}\right\} \supseteq\left\{B^{(1,3)} A^{(1,2)}\right\}$.
$\langle 9\rangle \quad$ Both $r(B)=p$ and $\mathscr{R}\left(A^{*}\right) \supseteq \mathscr{R}(B)$.
$\langle 8\rangle \quad\left\{M^{(1,2,4)}\right\} \supseteq\left\{B^{(1,3)} A^{(1)}\right\}$.

(g) The following 3 statements are equivalent:
$\langle 1\rangle \quad\left\{M^{(1,2,4)}\right\} \supseteq\left\{B^{(1,2,4)} A^{(1,3,4)}\right\}$.
$\langle 2\rangle \quad\left\{M^{(1,2,4)}\right\} \supseteq\left\{B^{(1,2,4)} A^{(1,4)}\right\}$.
$\langle 3\rangle \quad$ Either $\mathscr{R}\left(A^{*}\right) \supseteq \mathscr{R}(B)$ or $\left\{r(A)=m\right.$ and $\left.\mathscr{R}\left(B M^{*}\right)=\mathscr{R}\left(A^{*}\right)\right\}$.

(h) The following 3 statements are equivalent:
〈1〉 $\left\{M^{(1,2,4)}\right\} \supseteq\left\{B^{(1,4)} A^{\dagger}\right\}$.
$\langle 2\rangle \quad\left\{M^{(1,2,4)}\right\} \supseteq\left\{B^{(1,4)} A^{(1,2,4)}\right\}$.
$\langle 3\rangle \quad$ Either $\mathscr{R}\left(B M^{*}\right)=\mathscr{R}\left(A^{*}\right)$ or $\left\{r(B)=p\right.$ and $\left.\mathscr{R}\left(A^{*}\right) \supseteq \mathscr{R}(B)\right\}$.

(i) The following 3 statements are equivalent:
$\langle 1\rangle \quad\left\{M^{(1,2,4)}\right\} \supseteq\left\{B^{(1)} A^{\dagger}\right\}$.
$\langle 2\rangle \quad\left\{M^{(1,2,4)}\right\} \supseteq\left\{B^{(1)} A^{(1,2,4)}\right\}$
$\langle 3\rangle \quad$ Either $\left\{r(B)=p\right.$ and $\left.\mathscr{R}\left(B M^{*}\right)=\mathscr{R}\left(A^{*}\right)\right\}$ or $\left\{r(B)=p\right.$ and $\left.\mathscr{R}\left(A^{*}\right) \supseteq \mathscr{R}(B)\right\}$. 
(j) The following 5 statements are equivalent:
$\langle 1\rangle \quad\left\{M^{(1,2,4)}\right\} \supseteq\left\{B^{(1,3,4)} A^{(1,2,3)}\right\}$.
$\langle 3\rangle \quad\left\{M^{(1,2,4)}\right\} \supseteq\left\{B^{(1,3,4)} A^{(1,3)}\right\}$.
$\langle 2\rangle \quad\left\{M^{(1,2,4)}\right\} \supseteq\left\{B^{(1,3,4)} A^{(1,2)}\right\}$.
$\langle 5\rangle \quad$ Either $r(\bar{M})=n$ or $\left\{r(B)=p\right.$ and $\left.\mathscr{R}\left(A^{*}\right) \supseteq \mathscr{R}(B)\right\}$.

(k) The following 3 statements are equivalent:
$\langle 1\rangle \quad\left\{M^{(1,2,4)}\right\} \supseteq\left\{B^{\dagger} A^{(1,3,4)}\right\}$.
$\langle 2\rangle \quad\left\{M^{(1,2,4)}\right\} \supseteq\left\{B^{\dagger} A^{(1,4)}\right\}$.
$\langle 3\rangle \quad$ Either $\mathscr{R}\left(A^{*}\right) \supseteq \mathscr{R}(B)$ or $\left\{r(A)=m\right.$ and $\left.\mathscr{R}\left(B M^{*}\right) \subseteq \mathscr{R}\left(A^{*}\right)\right\}$.

(1) The following 3 statements are equivalent:
$\langle 1\rangle \quad\left\{M^{(1,2,4)}\right\} \supseteq\left\{B^{(1,2,3)} A^{(1,3,4)}\right\}$.
$\langle 3\rangle \quad$ Either $\left\{r(B)=p\right.$ and $\left.\mathscr{R}\left(A^{*}\right) \supseteq \mathscr{R}(B)\right\}$
$\langle 2\rangle \quad\left\{M^{(1,2,4)}\right\} \supseteq\left\{B^{(1,2,3)} A^{(1,4)}\right\}$.
or $\left\{r(A)=m, r(B)=p\right.$, and $\left.\mathscr{R}\left(B M^{*}\right) \subseteq \mathscr{R}\left(A^{*}\right)\right\}$.

(m) The following 3 statements are equivalent:
$\langle 1\rangle \quad\left\{M^{(1,2,4)}\right\} \supseteq\left\{B^{(1,3,4)} A^{\dagger}\right\}$.
$\langle 2\rangle \quad\left\{M^{(1,2,4)}\right\} \supseteq\left\{B^{(1,3,4)} A^{(1,2,4)}\right\}$.
$\langle 3\rangle \quad$ Either $\mathscr{R}\left(B M^{*}\right)=\mathscr{R}\left(A^{*}\right)$ or $\left\{r(B)=p\right.$ and $\left.\mathscr{R}\left(B M^{*}\right) \subseteq \mathscr{R}\left(A^{*}\right)\right\}$.

(n) The following 3 statements are equivalent:
〈1) $\quad\left\{M^{(1,2,4)}\right\} \supseteq\left\{B^{(1,4)} A^{(1,3,4)}\right\}$.
$\langle 2\rangle \quad\left\{M^{(1,2,4)}\right\} \supseteq\left\{B^{(1,4)} A^{(1,4)}\right\}$.
$\langle 3\rangle \quad$ Both $r(M)=\min \{m, n, p\}$ and $\mathscr{R}\left(B M^{*}\right) \subseteq \mathscr{R}\left(A^{*}\right)$.

(o) The following 3 statements are equivalent:
$\langle 1\rangle \quad\left\{M^{(1,2,4)}\right\} \supseteq\left\{B^{(1)} A^{(1,3,4)}\right\}$.
$\langle 2\rangle \quad\left\{M^{(1,2,4)}\right\} \supseteq\left\{B^{(1)} A^{(1,4)}\right\}$.
$\langle 3\rangle \quad r(B)=p, r(M)=\min \{m, n, p\}$, and $\mathscr{R}\left(B M^{*}\right) \subseteq \mathscr{R}\left(A^{*}\right)$.

(p) The following 3 statements are equivalent:
$\langle 1\rangle \quad\left\{M^{(1,2,4)}\right\} \supseteq\left\{B^{(1,3,4)} A^{(1,3,4)}\right\}$.
$\langle 2\rangle \quad\left\{M^{(1,2,4)}\right\} \supseteq\left\{B^{(1,3,4)} A^{(1,4)}\right\}$.
$\langle 3\rangle \quad$ Both $\mathscr{R}\left(B M^{*}\right) \subseteq \mathscr{R}\left(A^{*}\right)$ and $r(M)=\min \{m, n, p, t\}$.

(q) The following 3 statements are equivalent:
$\langle 1\rangle \quad\left\{M^{(1,2,4)}\right\} \supseteq\left\{B^{(1,3)} A^{(1,3,4)}\right\}$.
$\langle 2\rangle \quad\left\{M^{(1,2,4)}\right\} \supseteq\left\{B^{(1,3)} A^{(1,4)}\right\}$.
$\langle 3\rangle \quad r(B)=p, \mathscr{R}\left(B M^{*}\right) \subseteq \mathscr{R}\left(A^{*}\right)$, and $r(M)=\min \{m, p, t\}$.

(r) The following 9 statements are equivalent:
$\langle 1\rangle \quad\left\{M^{(1,2,4)}\right\} \supseteq\left\{B^{(1,2,4)} A^{(1,2,3)}\right\}$.
$\langle 2\rangle \quad\left\{M^{(1,2,4)}\right\} \supseteq\left\{B^{(1,2,4)} A^{(1,2)}\right\}$.
$\langle 3\rangle \quad\left\{M^{(1,2,4)}\right\} \supseteq\left\{B^{(1,4)} A^{(1,2,3)}\right\}$.
$\langle 4\rangle \quad\left\{M^{(1,2,4)}\right\} \supseteq\left\{B^{(1,4)} A^{(1,2)}\right\}$.
$\langle 5\rangle \quad\left\{M^{(1,2,4)}\right\} \supseteq\left\{B^{(1,2,4)} A^{(1,3)}\right\}$.
$\langle 6\rangle \quad\left\{M^{(1,2,4)}\right\} \supseteq\left\{B^{(1,2,4)} A^{(1)}\right\}$.
$\langle 7\rangle \quad\left\{M^{(1,2,4)}\right\} \supseteq\left\{B^{(1,4)} A^{(1,3)}\right\}$.
$\langle 8\rangle \quad\left\{M^{(1,2,4)}\right\} \supseteq\left\{B^{(1,3)} A^{(1)}\right\}$.
$\langle 9\rangle \quad r(M)=n$.

(s) The following 9 statements are equivalent:
$\langle 1\rangle \quad\left\{M^{(1,2,4)}\right\} \supseteq\left\{B^{(1,2)} A^{(1,2,3)}\right\}$.
$\langle 2\rangle \quad\left\{M^{(1,2,4)}\right\} \supseteq\left\{B^{(1,2)} A^{(1,2)}\right\}$.
$\langle 3\rangle \quad\left\{M^{(1,2,4)}\right\} \supseteq\left\{B^{(1)} A^{(1,2,3)}\right\}$.
$\langle 4\rangle \quad\left\{M^{(1,2,4)}\right\} \supseteq\left\{B^{(1)} A^{(1,2)}\right\}$.
$\langle 5\rangle \quad\left\{M^{(1,2,4)}\right\} \supseteq\left\{B^{(1,2)} A^{(1,3)}\right\}$.
$\langle 6\rangle \quad\left\{M^{(1,2,4)}\right\} \supseteq\left\{B^{(1,2)} A^{(1)}\right\}$.
$\langle 7\rangle \quad\left\{M^{(1,2,4)}\right\} \supseteq\left\{B^{(1)} A^{(1,3)}\right\}$.
$\langle 8\rangle \quad\left\{M^{(1,2,4)}\right\} \supseteq\left\{B^{(1)} A^{(1)}\right\}$.

Theorem 10.3. Let $A \in \mathbb{C}^{m \times n}$ and $B \in \mathbb{C}^{n \times p}$ be given, and denote $M=A B$. Then the following results hold. 
(a) The following 5 statements are equivalent:
$\langle 1\rangle \quad\left\{M^{(1,3,4)}\right\} \ni B^{\dagger} A^{\dagger}$.
$\langle 2\rangle \quad\left\{M^{(1,3,4)}\right\} \supseteq\left\{B^{\dagger} A^{(1,3,4)}\right\}$.
$\langle 3\rangle \quad\left\{M^{(1,3,4)}\right\} \supseteq\left\{B^{(1,3,4)} A^{\dagger}\right\}$.
$\langle 4\rangle \quad\left\{M^{(1,3,4)}\right\} \supseteq\left\{B^{(1,3,4)} A^{(1,3,4)}\right\}$.
$\langle 5\rangle \quad$ Both $\mathscr{R}\left(A^{*} M\right) \subseteq \mathscr{R}(B)$ and $\mathscr{R}\left(B M^{*}\right) \subseteq \mathscr{R}\left(A^{*}\right)$.

(b) The following 5 statements are equivalent:
$\langle 1\rangle \quad\left\{M^{(1,3,4)}\right\} \supseteq\left\{B^{\dagger} A^{(1,2,4)}\right\}$.
$\langle 2\rangle \quad\left\{M^{(1,3,4)}\right\} \supseteq\left\{B^{\dagger} A^{(1,4)}\right\}$.
$\langle 3\rangle \quad\left\{M^{(1,3,4)}\right\} \supseteq\left\{B^{(1,3,4)} A^{(1,2,4)}\right\}$.
$\langle 4\rangle \quad\left\{M^{(1,3,4)}\right\} \supseteq\left\{B^{(1,3,4)} A^{(1,4)}\right\}$.
$\langle 5\rangle \quad r(A)=m, \mathscr{R}\left(A^{*} M\right) \subseteq \mathscr{R}(B)$, and $\mathscr{R}\left(B M^{*}\right) \subseteq \mathscr{R}\left(A^{*}\right)$.

(c) The following 3 statements are equivalent:
$\langle 1\rangle \quad\left\{M^{(1,3,4)}\right\} \supseteq\left\{B^{(1,2,4)} A^{\dagger}\right\}$.
$\langle 2\rangle \quad\left\{M^{(1,3,4)}\right\} \supseteq\left\{B^{(1,4)} A^{\dagger}\right\}$.
$\langle 3\rangle \mathscr{R}\left(B M^{*}\right)=\mathscr{R}\left(A^{*}\right)$.

(d) The following 3 statements are equivalent:
$\langle 1\rangle \quad\left\{M^{(1,3,4)}\right\} \supseteq\left\{B^{\dagger} A^{(1,2,3)}\right\}$.
$\langle 2\rangle \quad\left\{M^{(1,3,4)}\right\} \supseteq\left\{B^{\dagger} A^{(1,3)}\right\}$.
$\langle 3\rangle \quad \mathscr{R}\left(A^{*} M\right)=\mathscr{R}(B)$

(e) The following 3 statements are equivalent:
$\langle 1\rangle \quad\left\{M^{(1,3,4)}\right\} \supseteq\left\{B^{(1,2,4)} A^{(1,3,4)}\right\}$.
$\langle 3\rangle \quad$ Either $\left\{r(\bar{B})=n\right.$ and $\left.\mathscr{R}\left(B M^{*}\right) \subseteq \mathscr{R}\left(A^{*}\right)\right\}$
$\langle 2\rangle \quad\left\{M^{(1,3,4)}\right\} \supseteq\left\{B^{(1,4)} A^{(1,3,4)}\right\}$. or $\left\{r(A)=m\right.$ and $\left.\mathscr{R}\left(B M^{*}\right)=\mathscr{R}\left(A^{*}\right)\right\}$.

(f) The following 5 statements are equivalent:
$\langle 1\rangle \quad\left\{M^{(1,3,4)}\right\} \supseteq\left\{B^{(1,2,4)} A^{(1,2,4)}\right\}$.
$\langle 3\rangle \quad\left\{M^{(1,3,4)}\right\} \supseteq\left\{B^{(1,4)} A^{(1,2,4)}\right\}$.
$\langle 5\rangle \quad$ Both $r(A)=m$ and $\mathscr{R}\left(B M^{*}\right)=\mathscr{R}\left(A^{*}\right)$.
$\langle 2\rangle \quad\left\{M^{(1,3,4)}\right\} \supseteq\left\{B^{(1,2,4)} A^{(1,4)}\right\}$.
$\langle 4\rangle \quad\left\{M^{(1,3,4)}\right\} \supseteq\left\{B^{(1,4)} A^{(1,4)}\right\}$.

(g) The following 5 statements are equivalent:
$\langle 1\rangle \quad\left\{M^{(1,3,4)}\right\} \supseteq\left\{B^{(1,2,3)} A^{\dagger}\right\}$.
$\langle 3\rangle \quad\left\{M^{(1,3,4)}\right\} \supseteq\left\{B^{(1,3)} A^{\dagger}\right\}$.
$\langle 2\rangle \quad\left\{M^{(1,3,4)}\right\} \supseteq\left\{B^{(1,2,3)} A^{(1,3,4)}\right\}$.
$\langle 5\rangle \quad r(B)=p, \mathscr{R}\left(A^{*} M\right) \subseteq \mathscr{R}(B)$, and $\mathscr{R}\left(B M^{*}\right) \subseteq \mathscr{R}\left(A^{*}\right)$.

(h) The following 5 statements are equivalent:
$\langle 1\rangle \quad\left\{M^{(1,3,4)}\right\} \supseteq\left\{B^{(1,2,3)} A^{(1,2,4)}\right\}$.
$\langle 2\rangle \quad\left\{M^{(1,3,4)}\right\} \supseteq\left\{B^{(1,2,3)} A^{(1,4)}\right\}$.
$\langle 3\rangle \quad\left\{M^{(1,3,4)}\right\} \supseteq\left\{B^{(1,3)} A^{(1,2,4)}\right\}$.
〈4) $\left\{M^{(1,3,4)}\right\} \supseteq\left\{B^{(1,3)} A^{(1,4)}\right\}$.
$\langle 5\rangle \quad r(A)=m, r(B)=p, \mathscr{R}\left(A^{*} M\right) \subseteq \mathscr{R}(B)$, and $\mathscr{R}\left(B M^{*}\right) \subseteq \mathscr{R}\left(A^{*}\right)$.

(i) The following 3 statements are equivalent:
$\langle 1\rangle \quad\left\{M^{(1,3,4)}\right\} \supseteq\left\{B^{(1,2)} A^{\dagger}\right\}$.
$\langle 3\rangle \quad$ Both $r(B)=p$ and $\mathscr{R}\left(B M^{*}\right)=\mathscr{R}\left(A^{*}\right)$.
$\langle 2\rangle \quad\left\{M^{(1,3,4)}\right\} \supseteq\left\{B^{(1)} A^{\dagger}\right\}$.

(j) The following 3 statements are equivalent:
$\langle 1\rangle \quad\left\{M^{(1,3,4)}\right\} \supseteq\left\{B^{(1,2)} A^{(1,3,4)}\right\}$.
$\langle 3\rangle \quad$ Either $\left\{r(B)=n=p\right.$ and $\left.\mathscr{R}\left(B M^{*}\right) \subseteq \mathscr{R}\left(A^{*}\right)\right\}$
$\langle 2\rangle \quad\left\{M^{(1,3,4)}\right\} \supseteq\left\{B^{(1)} A^{(1,3,4)}\right\}$.$$
\text { or }\left\{r(A)=m, r(B)=p \text {, and } \mathscr{R}\left(B M^{*}\right)=\mathscr{R}\left(A^{*}\right)\right\} \text {. }
$$

(k) The following 5 statements are equivalent:
$\langle 1\rangle \quad\left\{M^{(1,3,4)}\right\} \supseteq\left\{B^{(1,2)} A^{(1,2,4)}\right\}$.
$\langle 3\rangle \quad\left\{M^{(1,3,4)}\right\} \supseteq\left\{B^{(1)} A^{(1,2,4)}\right\}$.
$\langle 5\rangle \quad r(A)=m, r(B)=p$, and $\mathscr{R}\left(B M^{*}\right)=\mathscr{R}\left(A^{*}\right)$.
$\langle 2\rangle \quad\left\{M^{(1,3,4)}\right\} \supseteq\left\{B^{(1,2)} A^{(1,4)}\right\}$.
$\langle 4\rangle \quad\left\{M^{(1,3,4)}\right\} \supseteq\left\{B^{(1)} A^{(1,4)}\right\}$. 
(1) The following 3 statements are equivalent:
$\langle 1\rangle \quad\left\{M^{(1,3,4)}\right\} \supseteq\left\{B^{(1,3)} A^{(1,2)}\right\}$.
$\langle 2\rangle \quad\left\{M^{(1,3,4)}\right\} \supseteq\left\{B^{(1,3)} A^{(1)}\right\}$.
$\langle 3\rangle \quad\left\{M^{(1,3,4)}\right\} \supseteq\left\{B^{(1,2,3)} A^{(1,2)}\right\}$.
$\langle 4\rangle \quad\left\{M^{(1,3,4)}\right\} \supseteq\left\{B^{(1,2,3)} A^{(1)}\right\}$.
$\langle 5\rangle \quad r(A)=m, r(B)=p$, and $\mathscr{R}\left(A^{*} M\right)=\mathscr{R}(B)$.

(m) The following 3 statements are equivalent:
$\langle 1\rangle \quad\left\{M^{(1,3,4)}\right\} \supseteq\left\{B^{\dagger} A^{(1,2)}\right\}$.
$\langle 3\rangle \quad$ Both $r(A)=m$ and $\mathscr{R}\left(B M^{*}\right)=\mathscr{R}\left(A^{*}\right)$.
$\langle 2\rangle \quad\left\{M^{(1,3,4)}\right\} \supseteq\left\{B^{\dagger} A^{(1)}\right\}$.

(n) The following 3 statements are equivalent:
$\langle 1\rangle \quad\left\{M^{(1,3,4)}\right\} \supseteq\left\{B^{(1,3,4)} A^{(1,2,3)}\right\}$.
$\langle 3\rangle$ Either $\left\{r(A)=n\right.$ and $\left.\mathscr{R}\left(A^{*} M\right) \subseteq \mathscr{R}\left(B^{*}\right)\right\}$ or $\left\{r(B)=p\right.$ and $\left.\mathscr{R}\left(A^{*} M\right)=\mathscr{R}(B)\right\}$.
$\langle 2\rangle \quad\left\{M^{(1,3,4)}\right\} \supseteq\left\{B^{(1,3,4)} A^{(1,3)}\right\}$.

(o) The following 3 statements are equivalent:

$$
\begin{aligned}
& \langle 1\rangle \quad\left\{M^{(1,3,4)}\right\} \supseteq\left\{B^{(1,3,4)} A^{(1,2)}\right\} . \quad\langle 2\rangle \quad\left\{M^{(1,3,4)}\right\} \supseteq\left\{B^{(1,3,4)} A^{(1)}\right\} . \\
& \langle 3\rangle \quad \text { Either }\left\{r(A)=m=n \text { and } \mathscr{R}\left(A^{*} M\right) \subseteq \mathscr{R}(B)\right\} \\
& \quad \text { or }\left\{r(A)=m, r(B)=p, \text { and } \mathscr{R}\left(A^{*} M\right)=\mathscr{R}(B)\right\} .
\end{aligned}
$$

(p) The following 5 statements are equivalent:
$\langle 1\rangle \quad\left\{M^{(1,3,4)}\right\} \supseteq\left\{B^{(1,2,3)} A^{(1,2,3)}\right\}$.
$\langle 3\rangle \quad\left\{M^{(1,3,4)}\right\} \supseteq\left\{B^{(1,3)} A^{(1,2,3)}\right\}$.
$\langle 2\rangle \quad\left\{M^{(1,3,4)}\right\} \supseteq\left\{B^{(1,2,3)} A^{(1,3)}\right\}$.
$\langle 5\rangle \quad$ Both $r(B)=p$ and $\mathscr{R}\left(A^{*} M\right)=\mathscr{R}(B)$.
$\langle 4\rangle \quad\left\{M^{(1,3,4)}\right\} \supseteq\left\{B^{(1,3)} A^{(1,3)}\right\}$.

(q) The following 5 statements are equivalent:
$\langle 1\rangle \quad\left\{M^{(1,3,4)}\right\} \supseteq\left\{B^{(1,2,4)} A^{(1,2,3)}\right\}$.
$\langle 3\rangle \quad\left\{M^{(1,3,4)}\right\} \supseteq\left\{B^{(1,4)} A^{(1,2,3)}\right\}$.
$\langle 2\rangle \quad\left\{M^{(1,3,4)}\right\} \supseteq\left\{B^{(1,2,4)} A^{(1,3)}\right\}$.
$\langle 5\rangle \quad r(M)=n$.
$\langle 4\rangle \quad\left\{M^{(1,3,4)}\right\} \supseteq\left\{B^{(1,4)} A^{(1,3)}\right\}$.

(r) The following 5 statements are equivalent:
$\langle 1\rangle \quad\left\{M^{(1,3,4)}\right\} \supseteq\left\{B^{(1,2,4)} A^{(1,2)}\right\}$.
$\langle 3\rangle \quad\left\{M^{(1,3,4)}\right\} \supseteq\left\{B^{(1,4)} A^{(1,2)}\right\}$.
$\langle 2\rangle \quad\left\{M^{(1,3,4)}\right\} \supseteq\left\{B^{(1,2,4)} A^{(1)}\right\}$.
$\langle 5\rangle \quad r(M)=m=n$.
〈4〉 $\left\{M^{(1,3,4)}\right\} \supseteq\left\{B^{(1,4)} A^{(1)}\right\}$.

(s) The following 5 statements are equivalent:
$\langle 1\rangle \quad\left\{M^{(1,3,4)}\right\} \supseteq\left\{B^{(1,2)} A^{(1,2,3)}\right\}$.
$\langle 3\rangle \quad\left\{M^{(1,3,4)}\right\} \supseteq\left\{B^{(1)} A^{(1,2,3)}\right\}$.
$\langle 2\rangle \quad\left\{M^{(1,3,4)}\right\} \supseteq\left\{B^{(1,2)} A^{(1,3)}\right\}$.
$\langle 5\rangle \quad r(M)=n=p$.
$\langle 4\rangle \quad\left\{M^{(1,3,4)}\right\} \supseteq\left\{B^{(1)} A^{(1,3)}\right\}$.

(t) The following 5 statements are equivalent:
$\langle 1\rangle \quad\left\{M^{(1,3,4)}\right\} \supseteq\left\{B^{(1,2)} A^{(1,2)}\right\}$.
$\langle 3\rangle \quad\left\{M^{(1,3,4)}\right\} \supseteq\left\{B^{(1)} A^{(1,2)}\right\}$.
$\langle 2\rangle \quad\left\{M^{(1,3,4)}\right\} \supseteq\left\{B^{(1,2)} A^{(1)}\right\}$.
$\langle 5\rangle \quad r(M)=m=n=p$.
〈4) $\quad\left\{M^{(1,3,4)}\right\} \supseteq\left\{B^{(1)} A^{(1)}\right\}$.

Since the product $B^{\dagger} A^{\dagger}$ is unique, the last 64 cases in (1.9) can be written as

$$
(A B)^{\dagger}=\left\{(A B)^{(1,2,3,4)}\right\} \supseteq\left\{B^{\left(s_{2}, \ldots, t_{2}\right)} A^{\left(s_{1}, \ldots, t_{1}\right)}\right\}
$$

for the eight commonly-used types of generalized inverses of matrices. From (4.100),

$$
\begin{aligned}
& (A B)^{\dagger}=\left\{(A B)^{(1,2,3,4)}\right\} \supseteq\left\{B^{\left(s_{2}, \ldots, t_{2}\right)} A^{\left(s_{1}, \ldots, t_{1}\right)}\right\} \\
& \Leftrightarrow B^{\left(s_{2}, \ldots, t_{2}\right)} A^{\left(s_{1}, \ldots, t_{1}\right)} \text { is invariant and }(A B)^{\dagger}=B^{\dagger} A^{\dagger} .
\end{aligned}
$$


The invariance property of $B^{\left(s_{2}, \ldots, t_{2}\right)} A^{\left(s_{1}, \ldots, t_{1}\right)}$ is characterized in Theorem 6.1. Also by the definition of the Moore-Penrose inverse,

$$
(A B)^{\dagger}=B^{\dagger} A^{\dagger} \Leftrightarrow\left\{(A B)^{(1,2,3)}\right\} \ni B^{\dagger} A^{\dagger} \text { and }\left\{(A B)^{(1,2,4)}\right\} \ni B^{\dagger} A^{\dagger} .
$$

Thus we obtain from Theorems 10.1(a) and 10.2(a) that

$$
(A B)^{\dagger}=B^{\dagger} A^{\dagger} \Leftrightarrow \mathscr{R}\left(A^{*} A B\right) \subseteq \mathscr{R}(B) \text { and } \mathscr{R}\left(B B^{*} A^{*}\right) \subseteq \mathscr{R}\left(A^{*}\right) .
$$

This fact was well known in the theory of generalized inverses, which was first established in [32]. Finally, combining Theorem 6.1 with (10.4), we obtain the following results.

Theorem 10.4. Let $A \in \mathbb{C}^{m \times n}$ and $B \in \mathbb{C}^{n \times p}$ be given. Then the following results hold.

$\langle 1\rangle(A B)^{\dagger}=B^{\dagger} A^{\dagger} \Leftrightarrow \mathscr{R}\left(A^{*} A B\right) \subseteq \mathscr{R}(B)$ and $\mathscr{R}\left(B B^{*} A^{*}\right) \subseteq \mathscr{R}\left(A^{*}\right)$.

$\langle 2\rangle(A B)^{\dagger}=B^{\dagger} A^{(1,3,4)}$ holds for all $A^{(1,3,4)} \Leftrightarrow\left\{r(A)=m, \mathscr{R}\left(A^{*} A B\right) \subseteq \mathscr{R}(B)\right.$, and $\left.\mathscr{R}\left(B B^{*} A^{*}\right) \subseteq \mathscr{R}\left(A^{*}\right)\right\}$ or $\mathscr{R}\left(A^{*} A B\right)=\mathscr{R}(B)$.

$\langle 3\rangle(A B)^{\dagger}=B^{\dagger} A^{(1,2,4)}$ holds for all $A^{(1,2,4)} \Leftrightarrow A B=0$ or $\left\{r(A)=m, \mathscr{R}\left(A^{*} A B\right) \subseteq \mathscr{R}(B)\right.$, and $\mathscr{R}\left(B B^{*} A^{*}\right) \subseteq$ $\left.\mathscr{R}\left(A^{*}\right)\right\}$.

$\langle 4\rangle(A B)^{\dagger}=B^{\dagger} A^{(1,2,3)}$ holds for all $A^{(1,2,3)} \Leftrightarrow A=0$ or $\mathscr{R}\left(A^{*} A B\right)=\mathscr{R}(B)$.

$\langle 5\rangle(A B)^{\dagger}=B^{\dagger} A^{(1,4)}$ holds for all $A^{(1,4)} \Leftrightarrow\left\{r(A)=m, \mathscr{R}\left(A^{*} A B\right) \subseteq \mathscr{R}(B)\right.$, and $\left.\mathscr{R}\left(B B^{*} A^{*}\right) \subseteq \mathscr{R}\left(A^{*}\right)\right\}$ or $B=0$.

$\langle 6\rangle(A B)^{\dagger}=B^{\dagger} A^{(1,3)}$ holds for $A^{(1,3)}$ all $\Leftrightarrow \mathscr{R}\left(A^{*} A B\right)=\mathscr{R}(B)$.

$\langle 7\rangle(A B)^{\dagger}=B^{\dagger} A^{(1,2)}$ holds for all $A^{(1,2)} \Leftrightarrow A=0$ or $B=0$ or $\left\{r(A)=m\right.$ and $\left.\mathscr{R}\left(A^{*} A B\right)=\mathscr{R}(B)\right\}$.

$\langle 8\rangle(A B)^{\dagger}=B^{\dagger} A^{(1)}$ holds for all $A^{(1)} \Leftrightarrow\left\{r(A)=m\right.$ and $\left.\mathscr{R}\left(A^{*} A B\right)=\mathscr{R}(B)\right\}$ or $B=0$.

$\langle 9\rangle(A B)^{\dagger}=B^{(1,3,4)} A^{\dagger}$ holds for all $B^{(1,3,4)} \Leftrightarrow\left\{r(B)=p, \mathscr{R}\left(A^{*} A B\right) \subseteq \mathscr{R}(B)\right.$, and $\left.\mathscr{R}\left(B B^{*} A^{*}\right) \subseteq \mathscr{R}\left(A^{*}\right)\right\}$ or $\mathscr{R}\left(B B^{*} A^{*}\right)=\mathscr{R}\left(A^{*}\right)$.

$\langle 10\rangle(A B)^{\dagger}=B^{(1,3,4)} A^{(1,3,4)}$ holds for all $B^{(1,3,4)}$ and $A^{(1,3,4)} \Leftrightarrow r(A)=r(B)=n$ or $\{r(A)=m, r(B)=p$, $\mathscr{R}\left(A^{*} A B\right) \subseteq \mathscr{R}(B)$, and $\left.\mathscr{R}\left(B B^{*} A^{*}\right) \subseteq \mathscr{R}\left(A^{*}\right)\right\}$ or $\left\{r(A)=m\right.$ and $\left.\mathscr{R}\left(B B^{*} A^{*}\right)=\mathscr{R}\left(A^{*}\right)\right\}$ or $\{r(B)=p$ and $\left.\mathscr{R}\left(A^{*} A B\right)=\mathscr{R}(B)\right\}$.

$\langle 11\rangle(A B)^{\dagger}=B^{(1,3,4)} A^{(1,2,4)}$ holds for all $B^{(1,3,4)}$ and $A^{(1,2,4)} \Leftrightarrow A=0$ or $\{A B=0$ and $r(B)=p\}$ or $\{r(A)=m$ and $r(B)=p, \mathscr{R}\left(A^{*} A B\right) \subseteq \mathscr{R}(B)$, and $\left.\mathscr{R}\left(B B^{*} A^{*}\right) \subseteq \mathscr{R}\left(A^{*}\right)\right\}$ or $\left\{r(A)=m\right.$ and $\left.\mathscr{R}\left(B B^{*} A^{*}\right)=\mathscr{R}\left(A^{*}\right)\right\}$.

$\langle 12\rangle(A B)^{\dagger}=B^{(1,3,4)} A^{(1,2,3)}$ holds for all $B^{(1,3,4)}$ and $A^{(1,2,3)} \Leftrightarrow A=0$ or $r(A)=r(B)=n$ or $\{r(B)=p$ and $\left.\mathscr{R}\left(A^{*} A B\right)=\mathscr{R}(B)\right\}$.

$\langle 13\rangle(A B)^{\dagger}=B^{(1,3,4)} A^{(1,4)}$ holds for all $B^{(1,3,4)}$ and $A^{(1,4)} \Leftrightarrow\left\{r(A)=m, r(B)=p, \mathscr{R}\left(A^{*} A B\right) \subseteq \mathscr{R}(B)\right.$, and $\left.\mathscr{R}\left(B B^{*} A^{*}\right) \subseteq \mathscr{R}\left(A^{*}\right)\right\}$ or $\left\{r(A)=m\right.$ and $\left.\mathscr{R}\left(B B^{*} A^{*}\right)=\mathscr{R}\left(A^{*}\right)\right\}$.

$\langle 14\rangle(A B)^{\dagger}=B^{(1,3,4)} A^{(1,3)}$ holds for all $B^{(1,3,4)}$ and $A^{(1,3)} \Leftrightarrow r(A)=r(B)=n$ or $\left\{r(B)=p\right.$ and $\mathscr{R}\left(A^{*} A B\right)=$ $\mathscr{R}(B)\}$.

$\langle 14\rangle(A B)^{\dagger}=B^{(1,3,4)} A^{(1,2)}$ holds for all $B^{(1,3,4)}$ and $A^{(1,2)} \Leftrightarrow A=0$ or $r(A)=r(B)=m=n$ or $\{A B=$ $0, r(A)=m$, and $r(B)=p\}$.

$\langle 15\rangle(A B)^{\dagger}=B^{(1,3,4)} A^{(1)}$ holds for all $B^{(1,3,4)}$ and $A^{(1)} \Leftrightarrow r(A)=r(B)=m=n$ or $\{r(A)=m, r(B)=p$, and $\left.\mathscr{R}\left(A^{*} A B\right)=\mathscr{R}(B)\right\}$.

$\langle 17\rangle(A B)^{\dagger}=B^{(1,2,4)} A^{\dagger}$ holds for all $B^{(1,2,4)} \Leftrightarrow B=0$ or $\mathscr{R}\left(B B^{*} A^{*}\right)=\mathscr{R}\left(A^{*}\right)$.

$\langle 18\rangle(A B)^{\dagger}=B^{(1,2,4)} A^{(1,3,4)}$ holds for all $B^{(1,2,4)}$ and $A^{(1,3,4)} \Leftrightarrow B=0$ or $r(A)=r(B)=n$ or $\{r(A)=m$ and $\left.\mathscr{R}\left(B B^{*} A^{*}\right)=\mathscr{R}\left(A^{*}\right)\right\}$.

$\langle 19\rangle(A B)^{\dagger}=B^{(1,2,4)} A^{(1,2,4)}$ holds for all $B^{(1,2,4)}$ and $A^{(1,2,4)} \Leftrightarrow A=0$ or $B=0$ or $\left\{r(A)=m\right.$ and $\mathscr{R}\left(B B^{*} A^{*}\right)=$ $\left.\mathscr{R}\left(A^{*}\right)\right\}$.

$\langle 20\rangle(A B)^{\dagger}=B^{(1,2,4)} A^{(1,2,3)}$ holds for all $B^{(1,2,4)}$ and $A^{(1,2,3)} \Leftrightarrow A=0$ or $B=0$ or $r(A)=r(B)=n$.

$\langle 21\rangle(A B)^{\dagger}=B^{(1,2,4)} A^{(1,4)}$ holds for all $B^{(1,2,4)}$ and $A^{(1,4)} \Leftrightarrow B=0$ or $\left\{r(A)=m\right.$ and $\left.\mathscr{R}\left(B B^{*} A^{*}\right)=\mathscr{R}\left(A^{*}\right)\right\}$. 
$\langle 22\rangle(A B)^{\dagger}=B^{(1,2,4)} A^{(1,3)}$ holds for all $B^{(1,2,4)}$ and $A^{(1,3)} \Leftrightarrow B=0$ or $r(A)=r(B)=n$.

$\langle 23\rangle(A B)^{\dagger}=B^{(1,2,4)} A^{(1,2)}$ holds for all $B^{(1,2,4)}$ and $A^{(1,2)} \Leftrightarrow A=0$ or $B=0$ or $r(A)=r(B)=m=n$.

$\langle 24\rangle(A B)^{\dagger}=B^{(1,2,4)} A^{(1)}$ holds for all $B^{(1,2,4)}$ and $A^{(1)} \Leftrightarrow B=0$ or $r(A)=r(B)=m=n$.

$\langle 25\rangle(A B)^{\dagger}=B^{(1,2,3)} A^{\dagger}$ holds for all $B^{(1,2,3)} \Leftrightarrow A=0$ or $B=0$ or $\left\{(B)=p, \mathscr{R}\left(A^{*} A B\right) \subseteq \mathscr{R}(B)\right.$, and $\left.\mathscr{R}\left(B B^{*} A^{*}\right) \subseteq \mathscr{R}\left(A^{*}\right)\right\}$.

$\langle 25\rangle(A B)^{\dagger}=B^{(1,2,3)} A^{(1,3,4)}$ is invariant $\Leftrightarrow B=0\left\{A B=0\right.$ and $r(A)=m, \mathscr{R}\left(A^{*} A B\right) \subseteq \mathscr{R}(B)$, and $\left.\mathscr{R}\left(B B^{*} A^{*}\right) \subseteq \mathscr{R}\left(A^{*}\right)\right\}$ or $\left\{r(A)=m\right.$ and $r(B)=p, \mathscr{R}\left(A^{*} A B\right) \subseteq \mathscr{R}(B)$, and $\left.\mathscr{R}\left(B B^{*} A^{*}\right) \subseteq \mathscr{R}\left(A^{*}\right)\right\}$ or $\left\{r(B)=p\right.$ and $\left.\mathscr{R}\left(A^{*} A B\right)=\mathscr{R}(B)\right\}$.

$\langle 27\rangle(A B)^{\dagger}=B^{(1,2,3)} A^{(1,2,4)}$ holds for all $B^{(1,2,3)}$ and $A^{(1,2,4)} \Leftrightarrow A B=0$ or $\left\{r(A)=m, r(B)=p, \mathscr{R}\left(A^{*} A B\right) \subseteq\right.$ $\mathscr{R}(B)$, and $\left.\mathscr{R}\left(B B^{*} A^{*}\right) \subseteq \mathscr{R}\left(A^{*}\right)\right\}$.

$\langle 28\rangle(A B)^{\dagger}=B^{(1,2,3)} A^{(1,2,3)}$ holds for all $B^{(1,2,3)}$ and $A^{(1,2,3)} \Leftrightarrow A=0$ or $B=0$ or $\left\{r(B)=p\right.$ and $\mathscr{R}\left(A^{*} A B\right)=$ $\mathscr{R}(B)\}$.

$\langle 29\rangle(A B)^{\dagger}=B^{(1,2,3)} A^{(1,4)}$ holds for all $B^{(1,2,3)}$ and $A^{(1,4)} \Leftrightarrow B=0$ or $\{A B=0$ and $r(A)=m\}$ or $\{r(A)=m$, $r(B)=p, \mathscr{R}\left(A^{*} A B\right) \subseteq \mathscr{R}(B)$, and $\left.\mathscr{R}\left(B B^{*} A^{*}\right) \subseteq \mathscr{R}\left(A^{*}\right)\right\}$.

$\langle 30\rangle(A B)^{\dagger}=B^{(1,2,3)} A^{(1,3)}$ holds for all $B^{(1,2,3)}$ and $A^{(1,3)} \Leftrightarrow B=0$ or $r(B)=p$ and $\mathscr{R}\left(A^{*} A B\right)=\mathscr{R}(B)$.

$\langle 31\rangle(A B)^{\dagger}=B^{(1,2,3)} A^{(1,2)}$ holds for all $B^{(1,2,3)}$ and $A^{(1,2)} \Leftrightarrow A=0$ or $B=0$ or $\{r(A)=m, r(B)=p$, and $\left.\mathscr{R}\left(A^{*} A B\right)=\mathscr{R}(B)\right\}$.

$\langle 32\rangle(A B)^{\dagger}=B^{(1,2,3)} A^{(1)}$ holds for all $B^{(1,2,3)}$ and $A^{(1)} \Leftrightarrow B=0$ or $\left\{r(A)=m, r(B)=p\right.$, and $\mathscr{R}\left(A^{*} A B\right)=$ $\mathscr{R}(B)\}$.

$\langle 33\rangle(A B)^{\dagger}=B^{(1,4)} A^{\dagger}$ holds for all $B^{(1,4)} \Leftrightarrow \mathscr{R}\left(B B^{*} A^{*}\right)=\mathscr{R}\left(A^{*}\right)$.

$\langle 34\rangle(A B)^{\dagger}=B^{(1,4)} A^{(1,3,4)}$ holds for all $B^{(1,4)}$ and $A^{(1,3,4)} \Leftrightarrow$ either $r(A)=r(B)=n$ or $\{r(A)=m$ and $\left.\mathscr{R}\left(B B^{*} A^{*}\right)=\mathscr{R}\left(A^{*}\right)\right\}$.

$\langle 35\rangle(A B)^{\dagger}=B^{(1,4)} A^{(1,2,4)}$ holds for all $B^{(1,4)}$ and $A^{(1,2,4)} \Leftrightarrow A=0\left\{r(A)=m\right.$ and $\left.\mathscr{R}\left(B B^{*} A^{*}\right)=\mathscr{R}\left(A^{*}\right)\right\}$.

$\langle 36\rangle(A B)^{\dagger}=B^{(1,4)} A^{(1,2,3)}$ holds for all $B^{(1,4)}$ and $A^{(1,2,3)} \Leftrightarrow A=0$ or $r(A)=r(B)=n$.

$\langle 37\rangle(A B)^{\dagger}=B^{(1,4)} A^{(1,4)}$ holds for all $B^{(1,4)}$ and $A^{(1,4)} \Leftrightarrow r(A)=m$ and $\mathscr{R}\left(B B^{*} A^{*}\right)=\mathscr{R}\left(A^{*}\right)$.

$\langle 38\rangle(A B)^{\dagger}=B^{(1,4)} A^{(1,3)}$ holds for all $B^{(1,4)}$ and $A^{(1,3)} \Leftrightarrow r(A)=r(B)=n$.

$\langle 39\rangle(A B)^{\dagger}=B^{(1,4)} A^{(1,2)}$ holds for all $B^{(1,4)}$ and $A^{(1,2)} \Leftrightarrow A=0$ or $r(A)=r(B)=m=n$.

$\langle 40\rangle(A B)^{\dagger}=B^{(1,4)} A^{(1)}$ holds for all $B^{(1,4)}$ and $A^{(1)} \Leftrightarrow r(A)=r(B)=m=n$.

$\langle 41\rangle(A B)^{\dagger}=B^{(1,3)} A^{\dagger}$ holds for all $B^{(1,3)} \Leftrightarrow r(B)=p, \mathscr{R}\left(A^{*} A B\right) \subseteq \mathscr{R}(B)$, and $\mathscr{R}\left(B B^{*} A^{*}\right) \subseteq \mathscr{R}\left(A^{*}\right)$.

$\langle 42\rangle(A B)^{\dagger}=B^{(1,3)} A^{(1,3,4)}$ holds for all $B^{(1,3)}$ and $A^{(1,3,4)} \Leftrightarrow\left\{r(A)=m, r(B)=p, \mathscr{R}\left(A^{*} A B\right) \subseteq \mathscr{R}(B)\right.$, and $\left.\mathscr{R}\left(B B^{*} A^{*}\right) \subseteq \mathscr{R}\left(A^{*}\right)\right\}$ or $\left\{r(B)=p\right.$ and $\left.\mathscr{R}\left(A^{*} A B\right)=\mathscr{R}(B)\right\}$.

$\langle 43\rangle(A B)^{\dagger}=B^{(1,3)} A^{(1,2,4)}$ holds for all $B^{(1,3)}$ and $A^{(1,2,4)} \Leftrightarrow A=0$ or $\{A B=0$ and $r(B)=p\}$ or $\{r(A)=m$, $r(B)=p, \mathscr{R}\left(A^{*} A B\right) \subseteq \mathscr{R}(B)$, and $\left.\mathscr{R}\left(B B^{*} A^{*}\right) \subseteq \mathscr{R}\left(A^{*}\right)\right\}$.

$\langle 44\rangle(A B)^{\dagger}=B^{(1,3)} A^{(1,2,3)}$ holds for all $B^{(1,3)}$ and $A^{(1,2,3)} \Leftrightarrow A=0$ or $\left\{r(B)=p\right.$ and $\left.\mathscr{R}\left(A^{*} A B\right)=\mathscr{R}(B)\right\}$.

$\langle 45\rangle(A B)^{\dagger}=B^{(1,3)} A^{(1,4)}$ is holds for all $B^{(1,3)}$ and $A^{(1,4)} \Leftrightarrow r(A)=m$ and $r(B)=p, \mathscr{R}\left(A^{*} A B\right) \subseteq \mathscr{R}(B)$ and $\mathscr{R}\left(B B^{*} A^{*}\right) \subseteq \mathscr{R}\left(A^{*}\right)$.

$\langle 46\rangle(A B)^{\dagger}=B^{(1,3)} A^{(1,3)}$ holds for all $B^{(1,3)}$ and $A^{(1,3)} \Leftrightarrow r(B)=p$ and $\mathscr{R}\left(A^{*} A B\right)=\mathscr{R}(B)$.

$\langle 47\rangle(A B)^{\dagger}=B^{(1,3)} A^{(1,2)}$ holds for all $B^{(1,3)}$ and $A^{(1,2)} \Leftrightarrow A=0$ or $\left\{r(A)=m, r(B)=p\right.$, and $\mathscr{R}\left(A^{*} A B\right)=$ $\mathscr{R}(B)\}$.

$\langle 48\rangle(A B)^{\dagger}=B^{(1,3)} A^{(1)}$ holds for all $B^{(1,3)}$ and $A^{(1)} \Leftrightarrow r(A)=m, r(B)=p$, and $\mathscr{R}\left(A^{*} A B\right)=\mathscr{R}(B)$.

$\langle 49\rangle(A B)^{\dagger}=B^{(1,2)} A^{\dagger}$ holds for all $B^{(1,2)} \Leftrightarrow A=0$ or $B=0$ or $\left\{r(B)=p\right.$ and $\left.\mathscr{R}\left(B B^{*} A^{*}\right)=\mathscr{R}\left(A^{*}\right)\right\}$. 
$\langle 49\rangle(A B)^{\dagger}=B^{(1,2)} A^{(1,3,4)}$ holds for all $B^{(1,2)}$ and $A^{(1,3,4)} \Leftrightarrow B=0$ or $r(A)=r(B)=n=p$ or $\{A B=0, r(A)=$ $m$, and $r(B)=p\}$.

$\langle 51\rangle(A B)^{\dagger}=B^{(1,2)} A^{(1,2,4)}$ holds for all $B^{(1,2)}$ and $A^{(1,2,4)} \Leftrightarrow A=0$ or $B=0$ or $\{r(A)=m, r(B)=p$, and $\left.\mathscr{R}\left(B B^{*} A^{*}\right)=\mathscr{R}\left(A^{*}\right)\right\}$.

$\langle 52\rangle(A B)^{\dagger}=B^{(1,2)} A^{(1,2,3)}$ holds for all $B^{(1,2)}$ and $A^{(1,2,3)} \Leftrightarrow A=0$ or $B=0$ or $r(A)=r(B)=n=p$.

$\langle 53\rangle B^{(1,2)} A^{(1,3)}$ is invariant $\Leftrightarrow B=0$ or $r(A)=r(B)=n=p$.

$\langle 53\rangle(A B)^{\dagger}=B^{(1,2)} A^{(1,4)}$ holds for all $B^{(1,2)}$ and $A^{(1,4)} \Leftrightarrow B=0$ or $\left\{r(A)=m, r(B)=p\right.$, and $\mathscr{R}\left(B B^{*} A^{*}\right)=$ $\left.\mathscr{R}\left(A^{*}\right)\right\}$.

$\langle 54\rangle(A B)^{\dagger}=B^{(1,2)} A^{(1,3)}$ holds for all $B^{(1,2)}$ and $A^{(1,3)} \Leftrightarrow B=0$ or $r(A)=r(B)=n=p$.

$\langle 55\rangle(A B)^{\dagger}=B^{(1,2)} A^{(1,2)}$ holds for all $B^{(1,2)}$ and $A^{(1,2)} \Leftrightarrow A=0$ or $B=0$ or $r(A)=r(B)=m=n=p$.

$\langle 56\rangle(A B)^{\dagger}=B^{(1,2)} A^{(1)}$ holds for all $B^{(1,2)}$ and $A^{(1)} \Leftrightarrow B=0$ or $r(A)=r(B)=m=n=p$.

$\langle 57\rangle(A B)^{\dagger}=B^{(1)} A^{\dagger}$ holds for all $B^{(1)} \Leftrightarrow r(B)=p$ and $\mathscr{R}\left(B B^{*} A^{*}\right)=\mathscr{R}\left(A^{*}\right)$.

$\langle 58\rangle(A B)^{\dagger}=B^{(1)} A^{(1,3,4)}$ holds for all $B^{(1)}$ and $A^{(1,3,4)} \Leftrightarrow r(A)=r(B)=n=p$ or $\{r(A)=m, r(B)=p$, and $\left.\mathscr{R}\left(B B^{*} A^{*}\right)=\mathscr{R}\left(A^{*}\right)\right\}$.

$\langle 59\rangle(A B)^{\dagger}=B^{(1)} A^{(1,2,4)}$ holds for all $B^{(1)}$ and $A^{(1,2,4)} \Leftrightarrow A=0$ or $\left\{r(A)=m, r(B)=p\right.$, and $\mathscr{R}\left(B B^{*} A^{*}\right)=$ $\left.\mathscr{R}\left(A^{*}\right)\right\}$.

$\langle 60\rangle(A B)^{\dagger}=B^{(1)} A^{(1,2,3)}$ holds for all $B^{(1)}$ and $A^{(1,2,3)} \Leftrightarrow r(A)=r(B)=n=p$.

$\langle 61\rangle(A B)^{\dagger}=B^{(1)} A^{(1,4)}$ holds for all $\Leftrightarrow B^{(1)}$ and $A^{(1,4)} \Leftrightarrow r(A)=m, r(B)=p$, and $\mathscr{R}\left(B B^{*} A^{*}\right)=\mathscr{R}\left(A^{*}\right)$.

$\langle 62\rangle(A B)^{\dagger}=B^{(1)} A^{(1,3)}$ holds for all $B^{(1)}$ and $A^{(1,3)} \Leftrightarrow r(A)=r(B)=n=p$.

$\langle 63\rangle(A B)^{\dagger}=B^{(1)} A^{(1,2)}$ holds for all $B^{(1)}$ and $A^{(1,2)} \Leftrightarrow A=0$ or $r(A)=r(B)=m=n=p$.

$\langle 64\rangle(A B)^{\dagger}=B^{(1)} A^{(1)}$ holds for all $B^{(1)}$ and $A^{(1)} \Leftrightarrow r(A)=r(B)=m=n=p$.

\section{Miscellaneous results on ROLs}

As demonstrated in the preceding sections, the ROL $(A B)^{\dagger}=B^{\dagger} A^{\dagger}$ for the Moore-Penrose inverses is one of the most important forms in (1.7), while both 10.3 and (10.4) show that $(A B)^{\dagger}=B^{\dagger} A^{\dagger}$ has essential links with other types of the ROLs in (1.7), and can be characterized by many equivalent statements. In this section, we reconsider this ROL and present a bunch of necessary and sufficient conditions for the ROL to hold.

Lemma 11.1. Let $A \in \mathbb{C}^{m \times n}$ and $B \in \mathbb{C}^{n \times p}$, and denote $M=A B$. Then the following 11 statements are equivalent:

$\langle 1\rangle\left\{\left(A^{*} M\right)^{(1,3)}\right\} \ni M^{\dagger}\left(A^{*}\right)^{\dagger}$.

$\langle 2\rangle M^{\dagger}=\left(A^{\dagger} M\right)^{\dagger} A^{\dagger}$.

$\langle 3\rangle M^{\dagger}=\left(A^{*} M\right)^{\dagger} A^{*}$.

$\langle 4\rangle\left(A^{\dagger} M\right)^{\dagger}=M^{\dagger} A$.

$\langle 5\rangle A^{\dagger} M M^{\dagger} A$ is Hermitian, i.e., $\left(A^{\dagger} M M^{\dagger} A\right)^{*}=A^{\dagger} M M^{\dagger} A$.

$\langle 6\rangle M M^{\dagger}$ and $A A^{*}$ commute, i.e., $M M^{\dagger} A A^{*}=A A^{*} M M^{\dagger}$.

$\langle 7\rangle M B^{\dagger} A^{\dagger}$ is EP, i.e., $\mathscr{R}\left(M B^{\dagger} A^{\dagger}\right)=\mathscr{R}\left[\left(M B^{\dagger} A^{\dagger}\right)^{*}\right]$.

$\langle 8\rangle$ The range $R O L \mathscr{R}\left[\left(M^{*}\right)^{\dagger}\right]=\mathscr{R}\left[\left(A^{*}\right)^{\dagger}\left(B^{*}\right)^{\dagger}\right]$ for the Moore-Penrose inverse of matrix product holds.

$\langle 9\rangle r\left[A A^{*} M, M\right]=r(M)$.

$\langle 10\rangle \mathscr{R}\left(A A^{*} M\right)=\mathscr{R}(M)$.

$\langle 11\rangle r\left[A A^{*} M, M\right]=r(A)+r\left[A^{*} M, B\right]-r\left[A^{*}, B\right]$ and $r\left[A^{*} M, B\right]=r\left[A^{*}, B\right]+r(M)-r(A)$. 
Proof. By Theorem 9.4 $\langle 1\rangle$ and $\langle 15\rangle$, Result $\langle 1\rangle$ holds if and only if $r\left[A A^{*} M, M\right]=r(M)$, establishing the equivalence of $\langle 1\rangle,\langle 9\rangle$, and $\langle 10\rangle$.

The equivalence of $\langle 9\rangle$ and $\langle 11\rangle$ follows from $t_{4} \geq t_{5} \geq t_{6}$ in (4.29).

It is easy to verify that $(A B)^{\dagger},\left(A^{\dagger} A B\right)^{\dagger} A^{\dagger}$, and $\left(A^{*} A B\right)^{\dagger} A^{*}$ are $\{2\}$-inverses of $A B$. Then we obtain by 4.20 that

$$
\begin{aligned}
r\left[M^{\dagger}-\left(A^{\dagger} A B\right)^{\dagger} A^{\dagger}\right] & =r\left[\begin{array}{c}
M^{\dagger} \\
\left(A^{\dagger} M\right)^{\dagger} A^{\dagger}
\end{array}\right]+r\left[M^{\dagger},\left(A^{\dagger} M\right)^{\dagger} A^{\dagger}\right]-r\left(M^{\dagger}\right)-r\left[\left(A^{\dagger} M\right)^{\dagger} A^{\dagger}\right] \\
& =r\left[\begin{array}{c}
M^{*} \\
\left(A^{\dagger} M\right)^{*} A^{\dagger}
\end{array}\right]+r\left[M^{*},\left(A^{\dagger} M\right)^{*}\right]-2 r(M) \\
& =r\left[\begin{array}{c}
M^{*} A A^{*} \\
M^{*}
\end{array}\right]-r(M) \\
& =r\left[A A^{*} M, M\right]-r(M),
\end{aligned}
$$

and

$$
\begin{aligned}
r\left[M^{\dagger}-\left(A^{*} M\right)^{\dagger} A^{*}\right] & =r\left[\begin{array}{c}
M^{\dagger} \\
\left(A^{*} M\right)^{\dagger} A^{*}
\end{array}\right]+r\left[M^{\dagger},\left(A^{*} M\right)^{\dagger} A^{*}\right]-r\left(M^{\dagger}\right)-r\left[\left(A^{*} M\right)^{\dagger} A^{*}\right] \\
& =r\left[\begin{array}{c}
M^{*} \\
\left(A^{*} M\right)^{*} A^{*}
\end{array}\right]+r\left[M^{*},\left(A^{*} M\right)^{*}\right]-2 r(M) \\
& =r\left[\begin{array}{c}
M^{*} \\
\left(A A^{*} M\right)^{*}
\end{array}\right]-r(M) \\
& =r\left[A A^{*} M, M\right]-r(M) .
\end{aligned}
$$

It is also easy to verify that $\left(A^{\dagger} M\right)^{\dagger}$ and $M^{\dagger} A$ are $\{2\}$-inverses of $A^{\dagger} M$. Then we obtain by (4.20) that

$$
r\left[\left(A^{\dagger} M\right)^{\dagger}-M^{\dagger} A\right]=r\left[A A^{*} M, M\right]-r(M) .
$$

Setting all sides of 11.1 - 11.3 equal to zero leads to the equivalence of $\langle 2\rangle-\langle 4\rangle$, and $\langle 9\rangle$.

The rank of $\left(A^{\dagger} \overline{M M^{\dagger}} A\right)^{*}-A^{\dagger} M M^{\dagger} A$ is

$$
\begin{aligned}
r\left[\left(A^{\dagger} M M^{\dagger} A\right)^{*}-A^{\dagger} M M^{\dagger} A\right] & =r\left[A^{*} M M^{\dagger}\left(A^{\dagger}\right)^{*}-A^{\dagger} M M^{\dagger} A\right] \\
& =r\left(A A^{*} M M^{\dagger}-M M^{\dagger} A A^{*}\right) \\
& =2 r\left[A A^{*} M M^{\dagger}, M M^{\dagger}\right]-2 r\left(M M^{\dagger}\right) \quad(\text { by }(4.26)) \\
& =2 r\left[A A^{*} M, M\right]-2 r(M) .
\end{aligned}
$$

Setting all sides of 11.4 equal to zero leads to the equivalence of $\langle 5\rangle,\langle 6\rangle$, and $\langle 9\rangle$.

By 4.1 and Lemma 8.6,

$$
\begin{aligned}
r\left[M B^{\dagger} A^{\dagger},\left(M B^{\dagger} A^{\dagger}\right)^{*}\right] & =r\left[M,\left(A^{\dagger}\right)^{*} B\right]=r\left[A A^{*} M, M\right], \\
r\left[\left(M^{\dagger}\right)^{*},\left(A^{\dagger}\right)^{*}\left(B^{\dagger}\right)^{*}\right] & =r\left[M,\left(A^{\dagger}\right)^{*} B\right]=r\left[A A^{*} M, M\right] .
\end{aligned}
$$

Hence,

$$
\begin{aligned}
\mathscr{R}\left(M B^{\dagger} A^{\dagger}\right) & =\mathscr{R}\left[\left(M B^{\dagger} A^{\dagger}\right)^{*}\right] \Leftrightarrow r\left[\left(M^{\dagger}\right)^{*},\left(A^{\dagger}\right)^{*}\left(B^{\dagger}\right)^{*}\right]=r(M) \Leftrightarrow r\left[A A^{*} M, M\right]=r(M), \\
\mathscr{R}\left[\left(M^{\dagger}\right)^{*}\right] & =\mathscr{R}\left[\left(A^{\dagger}\right)^{*}\left(B^{\dagger}\right)^{*}\right] \Leftrightarrow r\left[\left(M^{\dagger}\right)^{*},\left(A^{\dagger}\right)^{*}\left(B^{\dagger}\right)^{*}\right]=r(M) \Leftrightarrow r\left[A A^{*} M, M\right]=r(M),
\end{aligned}
$$

establishing the equivalence of $\langle 7\rangle,\langle 8\rangle$, and $\langle 9\rangle$.

Lemma 11.2. Let $A \in \mathbb{C}^{m \times n}$ and $B \in \mathbb{C}^{n \times p}$, and denote $M=A B$. Then the following 11 statements are equivalent:

$\langle 1\rangle\left\{\left(M B^{*}\right)^{(1,4)}\right\} \ni\left(B^{*}\right)^{\dagger} M^{\dagger}$.

$\langle 2\rangle M^{\dagger}=B^{\dagger}\left(M B^{\dagger}\right)^{\dagger}$.

$\langle 3\rangle M^{\dagger}=B^{*}\left(M B^{*}\right)^{\dagger}$.

$\langle 4\rangle\left(M B^{\dagger}\right)^{\dagger}=B M^{\dagger}$.

$\langle 5\rangle B M^{\dagger} M B^{\dagger}$ is Hermitian, i.e., $\left(B M^{\dagger} M B^{\dagger}\right)^{*}=B M^{\dagger} M B^{\dagger}$. 
$\langle 6\rangle M^{\dagger} M$ and $B^{*} B$ commute, i.e., $M^{\dagger} M B^{*} B=B^{*} B M^{\dagger} M$.

$\langle 7\rangle B^{\dagger} A^{\dagger} M$ is EP, i.e., $\mathscr{R}\left(B^{\dagger} A^{\dagger} M\right)=\mathscr{R}\left[\left(B^{\dagger} A^{\dagger} M\right)^{*}\right]$.

$\langle 8\rangle$ The range $R O L \mathscr{R}\left(M^{\dagger}\right)=\mathscr{R}\left(B^{\dagger} A^{\dagger}\right)$ for the Moore-Penrose inverse of matrix product holds.

$\langle 9\rangle r\left[B^{*} B M^{*}, M^{*}\right]=r(M)$.

$\langle 10\rangle \mathscr{R}\left(B^{*} B M^{*}\right)=\mathscr{R}\left(M^{*}\right)$.

$\langle 11\rangle r\left[B^{*} B M^{*}, M^{*}\right]=r(B)+r\left[B M^{*}, A^{*}\right]-r\left[A^{*}, B\right]$ and $r\left[B M^{*}, A^{*}\right]=r\left[A^{*}, B\right]+r(M)-r(B)$.

We next collect/prove a family of known/novel necessary and sufficient conditions for the well-known case $(A B)^{\dagger}=B^{\dagger} A^{\dagger}$ in (1.7) to hold.

Theorem 11.3. Let $A \in \mathbb{C}^{m \times n}$ and $B \in \mathbb{C}^{n \times p}$, and denote $M=A B$. Then the following 73 statements are equivalent:

$\langle 1\rangle M^{\dagger}=B^{\dagger} A^{\dagger}$ and/or $M=\left(B^{\dagger} A^{\dagger}\right)^{\dagger}$.

$\langle 2\rangle M^{\dagger} \in\left\{B^{\dagger} A^{(1,3,4)}\right\}$.

$\langle 3\rangle M^{\dagger} \in\left\{B^{\dagger} A^{(1,2,4)}\right\}$.

〈4〉 $M^{\dagger} \in\left\{B^{\dagger} A^{(1,4)}\right\}$.

$\langle 5\rangle M^{\dagger} \in\left\{B^{(1,3,4)} A^{\dagger}\right\}$.

$\langle 6\rangle M^{\dagger} \in\left\{B^{(1,3,4)} A^{(1,3,4)}\right\}$.

$\langle 7\rangle M^{\dagger} \in\left\{B^{(1,3,4)} A^{(1,2,4)}\right\}$.

〈8〉 $M^{\dagger} \in\left\{B^{(1,3,4)} A^{(1,4)}\right\}$.

$\langle 9\rangle M^{\dagger} \in\left\{B^{(1,2,3)} A^{\dagger}\right\}$.

$\langle 10\rangle M^{\dagger} \in\left\{B^{(1,2,3)} A^{(1,3,4)}\right\}$.

〈11) $M^{\dagger} \in\left\{B^{(1,2,3)} A^{(1,2,4)}\right\}$.

$\langle 12\rangle M^{\dagger} \in\left\{B^{(1,2,3)} A^{(1,4)}\right\}$.

$\langle 13\rangle M^{\dagger} \in\left\{B^{(1,3)} A^{\dagger}\right\}$.

$\langle 14\rangle M^{\dagger} \in\left\{B^{(1,3)} A^{(1,3,4)}\right\}$.

$\langle 15\rangle M^{\dagger} \in\left\{B^{(1,3)} A^{(1,2,4)}\right\}$.

$\langle 16\rangle M^{\dagger} \in\left\{B^{(1,3)} A^{(1,4)}\right\}$.

$\langle 17\rangle\left\{M^{(1,3,4)}\right\} \supseteq\left\{B^{\dagger} A^{(1,3,4)}\right\}$.

$\langle 18\rangle\left\{M^{(1,3,4)}\right\} \supseteq\left\{B^{(1,3,4)} A^{\dagger}\right\}$.

$\langle 19\rangle\left\{M^{(1,3,4)}\right\} \supseteq\left\{B^{(1,3,4)} A^{(1,3,4)}\right\}$.

$\langle 20\rangle$ Both $\left\{M^{(1,3)}\right\} \ni B^{\dagger} A^{\dagger}$ and $\left\{M^{(1,4)}\right\} \ni B^{\dagger} A^{\dagger}$.

$\langle 21\rangle \operatorname{Both}\left\{M^{(1,3)}\right\} \supseteq\left\{B^{(1,3,4)} A^{\dagger}\right\}$ and $\left\{M^{(1,4)}\right\} \supseteq\left\{B^{\dagger} A^{(1,3,4)}\right\}$.

$\langle 22\rangle \operatorname{Both}\left\{M^{(1,3)}\right\} \supseteq\left\{B^{(1,2,3)} A^{\dagger}\right\}$ and $\left\{M^{(1,4)}\right\} \supseteq\left\{B^{\dagger} A^{(1,2,4)}\right\}$.

$\langle 23\rangle \operatorname{Both}\left\{M^{(1,3)}\right\} \supseteq\left\{B^{(1,3)} A^{\dagger}\right\}$ and $\left\{M^{(1,4)}\right\} \supseteq\left\{B^{\dagger} A^{(1,4)}\right\}$.

$\langle 24\rangle$ Both $\left\{M^{(1,3)}\right\} \supseteq\left\{B^{\dagger} A^{(1,3,4)}\right\}$ and $\left\{M^{(1,4)}\right\} \supseteq\left\{B^{(1,3,4)} A^{\dagger}\right\}$.

$\langle 25\rangle$ Both $\left\{M^{(1,3)}\right\} \supseteq\left\{B^{(1,3,4)} A^{(1,3,4)}\right\}$ and $\left\{M^{(1,4)}\right\} \supseteq\left\{B^{(1,3,4)} A^{(1,3,4)}\right\}$.

$\langle 26\rangle$ Both $\left\{M^{(1,3)}\right\} \supseteq\left\{B^{(1,2,3)} A^{(1,3,4)}\right\}$ and $\left\{M^{(1,4)}\right\} \supseteq\left\{B^{(1,3,4)} A^{(1,2,4)}\right\}$.

$\langle 27\rangle$ Both $\left\{M^{(1,3)}\right\} \supseteq\left\{B^{(1,3)} A^{(1,3,4)}\right\}$ and $\left\{M^{(1,4)}\right\} \supseteq\left\{B^{(1,3,4)} A^{(1,3)}\right\}$.

$\langle 28\rangle \operatorname{Both}\left\{M^{(1,3)}\right\} \supseteq\left\{B^{\dagger} A^{(1,2,3)}\right\}$ and $\left\{M^{(1,4)}\right\} \supseteq\left\{B^{(1,2,4)} A^{\dagger}\right\}$.

$\langle 29\rangle$ Both $\left\{M^{(1,3)}\right\} \supseteq\left\{B^{(1,3,4)} A^{(1,2,3)}\right\}$ and $\left\{M^{(1,4)}\right\} \supseteq\left\{B^{(1,2,4)} A^{(1,3,34)}\right\}$.

$\langle 30\rangle$ Both $\left\{M^{(1,3)}\right\} \supseteq\left\{B^{(1,2,3)} A^{(1,2,3)}\right\}$ and $\left\{M^{(1,4)}\right\} \supseteq\left\{B^{(1,2,4)} A^{(1,2,4)}\right\}$.

$\langle 31\rangle$ Both $\left\{M^{(1,3)}\right\} \supseteq\left\{B^{(1,3)} A^{(1,2,3)}\right\}$ and $\left\{M^{(1,4)}\right\} \supseteq\left\{B^{(1,2,4)} A^{(1,4)}\right\}$.

$\langle 32\rangle$ Both $\left\{M^{(1,3)}\right\} \supseteq\left\{B^{\dagger} A^{(1,3)}\right\}$ and $\left\{M^{(1,4)}\right\} \supseteq\left\{B^{(1,4)} A^{\dagger}\right\}$.

$\langle 33\rangle$ Both $\left\{M^{(1,3)}\right\} \supseteq\left\{B^{(1,3,4)} A^{(1,3)}\right\}$ and $\left\{M^{(1,4)}\right\} \supseteq\left\{B^{(1,4)} A^{(1,3,4)}\right\}$.

$\langle 34\rangle$ Both $\left\{M^{(1,3)}\right\} \supseteq\left\{B^{(1,2,3)} A^{(1,3)}\right\}$ and $\left\{M^{(1,4)}\right\} \supseteq\left\{B^{(1,4)} A^{(1,2,4)}\right\}$.

$\langle 35\rangle$ Both $\left\{M^{(1,3)}\right\} \supseteq\left\{B^{(1,3)} A^{(1,3)}\right\}$ and $\left\{M^{(1,4)}\right\} \supseteq\left\{B^{(1,4)} A^{(1,4)}\right\}$. 
$\langle 36\rangle\left\{\left(A^{*} M\right)^{(1)}\right\} \ni B^{\dagger}\left(A^{*} A\right)^{\dagger},\left\{\left(A^{*} M\right)^{(1,3)}\right\} \quad \ni M^{\dagger}\left(A^{*}\right)^{\dagger},\left\{\left(M B^{*}\right)^{(1)}\right\} \ni\left(B B^{*}\right)^{\dagger} A^{\dagger}$, and $\left\{\left(M B^{*}\right)^{(1,4)}\right\} \ni$ $\left(B^{*}\right)^{\dagger} M^{\dagger}$.

$\langle 37\rangle M^{\dagger}=B^{\dagger} A^{\dagger} M B^{\dagger} A^{\dagger} . \quad\langle 38\rangle B M^{\dagger} A=B B^{\dagger} A^{\dagger} A . \quad\langle 39\rangle B^{*} B M^{\dagger} A A^{*}=M^{*}$.

$\langle 40\rangle$ Both $M M^{\dagger}=M B^{\dagger} A^{\dagger}$ and $M^{\dagger} M=B^{\dagger} A^{\dagger} M$.

$\langle 41\rangle$ Both $M M^{\dagger} A=M B^{\dagger}$ and $A^{\dagger} M=B M^{\dagger} M$.

$\langle 42\rangle$ Both $B B^{\dagger} A^{*} A B=A^{*} A B$ and $A B B^{*} A^{\dagger} A=A B B^{*}$.

$\langle 43\rangle B B^{\dagger} A^{*} A B B^{*} A^{\dagger} A=B B^{\dagger} A^{*} A B B^{*}=A^{*} A B B^{*} A^{\dagger} A=A^{*} A B B^{*}$.

〈44>Both $M^{\dagger}=\left(A^{\dagger} A B\right)^{\dagger} A^{\dagger}$ and $\left(A^{\dagger} A B\right)^{\dagger}=B^{\dagger} A^{\dagger} A$.

$\langle 45\rangle$ Both $M^{\dagger}=B^{\dagger}\left(A B B^{\dagger}\right)^{\dagger}$ and $\left(A B B^{\dagger}\right)^{\dagger}=B B^{\dagger} A^{\dagger}$.

$\langle 46\rangle$ Both $M^{\dagger}=\left(A^{*} A B\right)^{\dagger} A^{*}$ and $\left(A^{*} A B\right)^{\dagger}=B^{\dagger}\left(A^{*} A\right)^{\dagger}$.

$\langle 47\rangle$ Both $M^{\dagger}=B^{*}\left(A B B^{*}\right)^{\dagger}$ and $\left(A B B^{*}\right)^{\dagger}=\left(B B^{*}\right)^{\dagger} A^{\dagger}$.

$\langle 48\rangle$ Both $M^{\dagger}=B^{\dagger}\left(A^{\dagger} A B B^{\dagger}\right)^{\dagger} A^{\dagger}$ and $\left(A^{\dagger} A B B^{\dagger}\right)^{\dagger}=B B^{\dagger} A^{\dagger} A$.

$\langle 49\rangle$ Both $M^{\dagger}=B^{*}\left(B B^{*}\right)^{k-1}\left[\left(A^{*} A\right)^{k}\left(B B^{*}\right)^{k}\right]^{\dagger}\left(A^{*} A\right)^{k-1} A^{*}$ and $\left[\left(A^{*} A\right)^{k}\left(B B^{*}\right)^{k}\right]^{\dagger}=\left[\left(B B^{*}\right)^{k}\right]^{\dagger}\left[\left(A^{*} A\right)^{k}\right]^{\dagger}$ for any integer $k \geq 1$.

$\langle 50\rangle$ Both $M^{\dagger}=B^{*} B\left(A A^{*} A B B^{*} B\right)^{\dagger} A A^{*}$ and $\left(A A^{*} A B B^{*} B\right)^{\dagger}=\left(B B^{*} B\right)^{\dagger}\left(A A^{*} A\right)^{\dagger}$.

$\langle 51\rangle$ Both $\left(A B B^{\dagger}\right)^{\dagger}=B B^{\dagger} A^{\dagger}$ and $\left(A^{\dagger} A B\right)^{\dagger}=B^{\dagger} A^{\dagger} A$.

$\langle 52\rangle$ Both $M B^{\dagger} A^{\dagger}$ and $B^{\dagger} A^{\dagger} M$ are orthogonal projectors.

$\langle 53\rangle$ Both $A E_{B} A^{\dagger}$ and $B^{\dagger} F_{A} B$ are orthogonal projectors.

〈54〉Both $M M^{\dagger} A=M B^{\dagger}$ and $B M^{\dagger} M=A^{\dagger} M$.

$\langle 55\rangle$ Both $A^{*} A B B^{\dagger}=B B^{\dagger} A^{*} A$ and $A^{\dagger} A B B^{*}=B B^{*} A^{\dagger} A$.

$\langle 56\rangle$ Both $M B^{\dagger} A^{\dagger} M=M$ and $M^{\dagger}=\left(A^{\dagger} M\right)^{\dagger} A^{\dagger}=B^{\dagger}\left(M B^{\dagger}\right)^{\dagger}$.

$\langle 57\rangle$ Both $M B^{\dagger} A^{\dagger} M=M$ and $M^{\dagger}=\left(A^{*} M\right)^{\dagger} A^{*}=B^{*}\left(M B^{*}\right)^{\dagger}$.

$\langle 58\rangle M B^{\dagger} A^{\dagger} M=M,\left(A^{\dagger} M\right)^{\dagger}=M^{\dagger} A$, and $\left(M B^{\dagger}\right)^{\dagger}=B M^{\dagger}$.

$\langle 59\rangle M B^{\dagger} A^{\dagger} M=M,\left(A^{\dagger} M M^{\dagger} A\right)^{*}=A^{\dagger} M M^{\dagger} A$, and $\left(B M^{\dagger} M B^{\dagger}\right)^{*}=B M^{\dagger} M B^{\dagger}$.

$\langle 60\rangle M B^{\dagger} A^{\dagger} M=M, M M^{\dagger} A A^{*}=A A^{*} M M^{\dagger}$, and $M^{\dagger} M B^{*} B=B^{*} B M^{\dagger} M$.

$\langle 61\rangle$ Both $\mathscr{R}\left(A^{*} A B B^{\dagger}\right)=\mathscr{R}\left(B B^{\dagger} A^{*} A\right)$ and $\mathscr{R}\left(A^{\dagger} A B B^{*}\right)=\mathscr{R}\left(B B^{*} A^{\dagger} A\right)$.

$\langle 62\rangle \mathscr{R}\left(A^{\dagger} A B B^{\dagger}\right)=\mathscr{R}\left(B B^{\dagger} A^{\dagger} A\right), \mathscr{R}\left(M^{\dagger}\right)=\mathscr{R}\left(B^{\dagger} A^{\dagger}\right)$, and $\mathscr{R}\left[\left(M^{*}\right)^{\dagger}\right]=\mathscr{R}\left[\left(A^{*}\right)^{\dagger}\left(B^{*}\right)^{\dagger}\right]$.

$\langle 63\rangle$ Both $\mathscr{R}\left(A^{\dagger} A B B^{\dagger}\right)=\mathscr{R}\left(B B^{*} A^{*} A\right)$ and $\mathscr{R}\left(B B^{\dagger} A^{\dagger} A\right)=\mathscr{R}\left(A^{*} A B B^{*}\right)$.

$\langle 64\rangle \mathscr{R}\left(A^{*} A B B^{*}\right)=\mathscr{R}\left(B B^{*} A^{*} A\right)$, i.e., $A^{*} A B B^{*}$ is $E P$.

$\langle 65\rangle$ Both $\mathscr{R}\left(A^{*} M\right) \subseteq \mathscr{R}(B)$ and $\mathscr{R}\left(B M^{*}\right) \subseteq \mathscr{R}\left(A^{*}\right)$.

$\langle 66\rangle$ Both $\mathscr{R}\left(A^{*} M\right)=\mathscr{R}\left(A^{*}\right) \cap \mathscr{R}(B)$ and $\mathscr{R}\left(B M^{*}\right)=\mathscr{R}(B) \cap \mathscr{R}\left(A^{*}\right)$.

$\langle 67\rangle$ Both $r\left[A^{*} M, B\right]=r(B)$ and $r\left[B M^{*}, A^{*}\right]=r(A)$.

$\langle 68\rangle r\left[A^{*}, B\right]=r(A)+r(B)-r(M), r\left[A A^{*} M, M\right]=r(M)$, and $r\left[B^{*} B M^{*}, M^{*}\right]=r(M)$.

$\langle 69\rangle r\left[\begin{array}{cc}M M^{*} M & M B^{*} B \\ A A^{*} M & M\end{array}\right]=r(M)$.

$\langle 70\rangle M B^{*} B M^{\dagger} A A^{*} M=M M^{*} M, r\left[A A^{*} M, M\right]=r(M)$, and $r\left[B^{*} B M^{*}, M^{*}\right]=r(M)$.

$\langle 71\rangle r\left[\begin{array}{cc}M M^{*} M & M B^{*} B \\ A A^{*} M & M\end{array}\right]=r\left[A^{*}, B\right]+2 r(M)-r(A)-r(B)$ and $r\left[A^{*} M, B\right]+r\left[B M^{*}, A^{*}\right]=r\left[A^{*}, B\right]+r(M)$. 
$\begin{aligned}\langle 72\rangle r\left[\begin{array}{cc}M M^{*} M & M B^{*} B \\ A A^{*} M & M\end{array}\right] & =r\left[A^{*} M, B\right]+r\left[B M^{*}, A^{*}\right]+r(A)+r(B)-2 r\left[A^{*}, B\right]-r(A B), r\left[A A^{*} M, M\right]=r(M), \\ \text { and } r\left[B^{*} B M^{*}, M^{*}\right] & =r(M) .\end{aligned}$

$\langle 73\rangle$ The matrix equation $B X A=A^{*} A B B^{*}$ is consistent.

Proof. Result $\langle 1\rangle$ obviously implies $\langle 2\rangle-\langle 16\rangle$ by Lemma 3.1(c). Conversely, if one of the following holds $M^{\dagger}=$ $B^{\dagger} A^{(1,3,4)}, M^{\dagger}=B^{\dagger} A^{(1,2,4)}, M^{\dagger}=B^{\dagger} A^{(1,4)}, M^{\dagger}=B^{(1,3,4)} A^{\dagger}, M^{\dagger}=B^{(1,3,4)} A^{(1,3,4)}, M^{\dagger}=B^{(1,3,4)} A^{(1,2,4)}, M^{\dagger}=$ $B^{(1,3,4)} A^{(1,4)}, M^{\dagger}=B^{(1,2,3)} A^{\dagger}, M^{\dagger}=B^{(1,2,3)} A^{(1,3,4)}, M^{\dagger}=B^{(1,2,3)} A^{(1,2,4)}, M^{\dagger}=B^{(1,2,3)} A^{(1,4)}, M^{\dagger}=B^{(1,3)} A^{\dagger}$, $M^{\dagger}=B^{(1,3)} A^{(1,3,4)}, M^{\dagger}=B^{(1,3)} A^{(1,2,4)}$, and $M^{\dagger}=B^{(1,3)} A^{(1,4)}$, then pre- and post-multiplying $B^{\dagger} B$ and $A A^{\dagger}$ to both sides of these equalities and applying (3.36), 3.38), and $B^{\dagger} B M^{\dagger} A A^{\dagger}=M^{\dagger}$ lead to

$$
\begin{aligned}
& M^{\dagger}=B^{\dagger} B M^{\dagger} A A^{\dagger}=B^{\dagger} B B^{\dagger} A^{(1,3,4)} A A^{\dagger}=B^{\dagger} A^{\dagger}, \\
& M^{\dagger}=B^{\dagger} B M^{\dagger} A A^{\dagger}=B^{\dagger} B B^{\dagger} A^{(1,2,4)} A A^{\dagger}=B^{\dagger} A^{\dagger}, \\
& M^{\dagger}=B^{\dagger} B M^{\dagger} A A^{\dagger}=B^{\dagger} B B^{\dagger} A^{(1,4)} A A^{\dagger}=B^{\dagger} A^{\dagger}, \\
& M^{\dagger}=B^{\dagger} B M^{\dagger} A A^{\dagger}=B^{\dagger} B B^{\dagger} B B^{(1,3,4)} A^{\dagger} A A^{\dagger}=B^{\dagger} A^{\dagger}, \\
& M^{\dagger}=B^{\dagger} B M^{\dagger} A A^{\dagger}=B^{(1,3,4)} A^{(1,3,4)} A A^{\dagger}=B^{\dagger} A^{\dagger}, \\
& M^{\dagger}=B^{\dagger} B M^{\dagger} A A^{\dagger}=B^{(1,3,4)} A^{(1,2,4)} A A^{\dagger}=B^{\dagger} A^{\dagger}, \\
& M^{\dagger}=B^{\dagger} B M^{\dagger} A A^{\dagger}=B^{(1,3,4)} A^{(1,4)} A A^{\dagger}=B^{\dagger} A^{\dagger}, \\
& M^{\dagger}=B^{\dagger} B M^{\dagger} A A^{\dagger}=B^{(1,2,3)} A^{\dagger} A A^{\dagger}=B^{\dagger} A^{\dagger}, \\
& M^{\dagger}=B^{\dagger} B M^{\dagger} A A^{\dagger}=B^{(1,2,3)} A^{(1,3,4)} A A^{\dagger}=B^{\dagger} A^{\dagger}, \\
& M^{\dagger}=B^{\dagger} B M^{\dagger} A A^{\dagger}=B^{(1,2,3)} A^{(1,2,4)} A A^{\dagger}=B^{\dagger} A^{\dagger}, \\
& M^{\dagger}=B^{\dagger} B M^{\dagger} A A^{\dagger}=B^{(1,2,3)} A^{(1,4)} A A^{\dagger}=B^{\dagger} A^{\dagger}, \\
& M^{\dagger}=B^{\dagger} B M^{\dagger} A A^{\dagger}=B^{(1,3)} A^{\dagger} A A^{\dagger}=B^{\dagger} A^{\dagger}, \\
& M^{\dagger}=B^{\dagger} B M^{\dagger} A A^{\dagger}=B^{(1,3)} A^{(1,3,4)} A A^{\dagger}=B^{\dagger} A^{\dagger}, \\
& M^{\dagger}=B^{\dagger} B M^{\dagger} A A^{\dagger}=B^{(1,3)} A^{(1,2,4)} A A^{\dagger}=B^{\dagger} A^{\dagger}, \\
& M^{\dagger}=B^{\dagger} B M^{\dagger} A A^{\dagger}=B^{(1,3)} A^{(1,4)} A A^{\dagger}=B^{\dagger} A^{\dagger} .
\end{aligned}
$$

Eqs. 11.5 11.19 show that each of $\langle 2\rangle-\langle 16\rangle$ implies $\langle 1\rangle$.

The equivalence of $\langle 1\rangle$ and $\langle 17\rangle-\langle 19\rangle$ follows from Theorem 10.3 (a).

The equivalence of $\langle 20\rangle-\langle 35\rangle$ and $\langle 67\rangle$ follows from Theorem 9.4(a), (b), (d), and (f), and Theorem 9.5(a), (b), (d), and (f).

The equivalence of $\langle 36\rangle$ and $\langle 68\rangle$ follows from Theorem 9.1 $\langle 78\rangle$ and $\langle 116\rangle$, Lemma 11.1 $\langle 1\rangle$ and $\langle 9\rangle$, and Lemma $11.2\langle 1\rangle$ and $\langle 9\rangle$.

Result $\langle 1\rangle$ obviously implies $\langle 37\rangle$. Conversely, pre-multiplying the equality in $\langle 37\rangle$ with $A B$ yields $A B M^{\dagger}=$ $A B B^{\dagger} A^{\dagger} A B B^{\dagger} A^{\dagger}=\left(A B B^{\dagger} A^{\dagger}\right)^{2}$, where $A B M^{\dagger}$ is idempotent. So that $\left(A B B^{\dagger} A^{\dagger}\right)^{2}=A B B^{\dagger} A^{\dagger}$, which is equivalent to $B^{\dagger} A^{\dagger} A B B^{\dagger} A^{\dagger}=B^{\dagger} A^{\dagger}$ by Theorem 9.1 $\langle 88\rangle$ and $\langle 93\rangle$. Thus, $\langle 37\rangle$ implies $\langle 1\rangle$.

Pre- and post-multiplying both sides of $\langle 1\rangle$ with $A$ and $B$ yields $\langle 38\rangle$. Conversely, pre- and post-multiplying both sides of $\langle 38\rangle$ with $A^{\dagger}$ and $B^{\dagger}$ yields $\langle 1\rangle$.

Pre- and post-multiplying both sides of $\langle 1\rangle$ with $A^{*}$ and $B^{*}$ yields $\langle 39\rangle$. Conversely, pre- and post-multiplying both sides of $\langle 39\rangle$ with $\left(A^{\dagger}\right)^{*}$ and $\left(B^{\dagger}\right)^{*}$ yields $\langle 1\rangle$.

The equivalence of $\langle 1\rangle$ and $\langle 40\rangle$ follows from Theorem 9.4(a) $\langle 1\rangle$ and $\langle 5\rangle$ and Theorem 9.5(a) $\langle 1\rangle$ and $\langle 5\rangle$.

The equivalence of $\langle 1\rangle$ and $\langle 41\rangle$ follows from Theorem 9.4(a) $\langle 1\rangle$ and $\langle 5\rangle$ and Theorem 9.5(a) $\langle 1\rangle$ and $\langle 5\rangle$.

The equivalence of $\langle 42\rangle$ and $\langle 67\rangle$ follows from Theorem 9.4(a) $\langle 8\rangle$ and $\langle 17\rangle$ and Theorem 9.5(a) $\langle 8\rangle$ and $\langle 17\rangle$.

The equivalence of $\langle 43\rangle$ and $\langle 67\rangle$ is derived from Lemma 5.2(d) and (e).

The equivalence of $\langle 44\rangle$ and $\langle 68\rangle$ follows from Lemma 11.1 $\langle 2\rangle$ and $\langle 9\rangle$ and Theorem 9.5(a) $\langle 9\rangle$ and $\langle 26\rangle$.

The equivalence of $\langle 45\rangle$ and $\langle 68\rangle$ follows from Lemma 11.2 $\langle 2\rangle$ and $\langle 9\rangle$ and Theorem 9.4(a) $\langle 9\rangle$ and $\langle 26\rangle$.

Applying the equivalence of $\langle 1\rangle$ and $\langle 65\rangle$ to the two equalities in $\langle 46\rangle$, we first see that

$$
\begin{aligned}
& M^{\dagger}=\left(A^{*} A B\right)^{\dagger} A^{*} \Leftrightarrow \mathscr{R}\left(A A^{*} M\right)=\mathscr{R}(M), \\
& \left(A^{*} A B\right)^{\dagger}=B^{\dagger}\left(A^{*} A\right)^{\dagger} \Leftrightarrow \operatorname{both} \mathscr{R}\left[\left(A A^{*}\right)^{2} A B\right] \subseteq \mathscr{R}(B) \text { and } \mathscr{R}\left(B B^{*} A^{*}\right) \subseteq \mathscr{R}\left(A^{*}\right) .
\end{aligned}
$$

Also by (8.19),

$$
\mathscr{R}\left(A A^{*} A B\right) \subseteq \mathscr{R}(B) \Rightarrow \mathscr{R}\left[\left(A A^{*}\right)^{2} A B\right] \subseteq \mathscr{R}\left(A A^{*} B\right) \Rightarrow \mathscr{R}\left[\left(A A^{*}\right)^{2} A B\right] \subseteq \mathscr{R}(B) .
$$


Thus if $\langle 1\rangle$ holds, combining $\langle 65\rangle$ and $\langle 68\rangle$ with 11.20, , 11.21, and 11.22 , we see that the two equalities in $\langle 46\rangle$ hold. Conversely, merging the two equalities in $\langle 46\rangle$ and simplifying yields the equality in $\langle 1\rangle$.

Merging the two equalities in $\langle 48\rangle$ and simplifying yields the equality in $\langle 1\rangle$. Conversely, notice that $B^{\dagger}\left(A^{\dagger} A B B^{\dagger}\right)^{\dagger} A^{\dagger}$ is a $\{2\}$-inverses of $A B$. Then we obtain by (4.20) that

$$
\begin{aligned}
r\left[M^{\dagger}-B^{\dagger}\left(A^{\dagger} A B B^{\dagger}\right)^{\dagger} A^{\dagger}\right]= & r\left[\begin{array}{c}
M^{\dagger} \\
B^{\dagger}\left(A^{\dagger} A B B^{\dagger}\right)^{\dagger} A^{\dagger}
\end{array}\right]+r\left[M^{\dagger}, B^{\dagger}\left(A^{\dagger} A B B^{\dagger}\right)^{\dagger} A^{\dagger}\right] \\
& -r\left(M^{\dagger}\right)-r\left[B^{\dagger}\left(A^{\dagger} A B B^{\dagger}\right)^{\dagger} A^{\dagger}\right] \\
& =r\left[\begin{array}{c}
M^{*} \\
\left(A^{\dagger} A B B^{\dagger}\right)^{*} A^{\dagger}
\end{array}\right]+r\left[M^{*},\left(A^{\dagger} A B B^{\dagger}\right)^{*}\right]-2 r(M) \\
& =r\left[\begin{array}{c}
M^{*} \\
B^{*} A^{\dagger}
\end{array}\right]+r\left[M^{*}, B^{\dagger}\left(A^{\dagger} A B B^{\dagger}\right)^{*}\right]-2 r(M) \\
& =r\left[\begin{array}{c}
M \\
M B^{*} B
\end{array}\right]+r\left[M, A A^{*} M\right]-2 r(M) .
\end{aligned}
$$

So that

$$
M^{\dagger}=B^{\dagger}\left(A^{\dagger} A B B^{\dagger}\right)^{\dagger} A^{\dagger} \Leftrightarrow r\left[A A^{*} M, M\right]=r(M) \text { and } r\left[B^{*} B M^{*}, M^{*}\right]=r(M) .
$$

Thus if $\langle 1\rangle$ holds, combining $\langle 68\rangle$ with 11.24 and Theorem 9.1 $\langle 98\rangle$ and $\langle 116\rangle$, we see that the two equalities in $\langle 48\rangle$ hold.

Merging the two equalities in $\langle 49\rangle$ and simplifying yields the equality in $\langle 1\rangle$. Conversely, notice that $B^{*}\left(B B^{*}\right)^{k-1}\left[\left(A^{*} A\right)^{k}\left(B B^{*}\right)^{k}\right]^{\dagger}\left(A^{*} A\right)^{k-1} A^{*}$ is a $\{2\}$-inverses of $A B$. Then we obtain by (4.20) that

$$
\begin{aligned}
r\{ & \left.M^{\dagger}-B^{*}\left(B B^{*}\right)^{k-1}\left[\left(A^{*} A\right)^{k}\left(B B^{*}\right)^{k}\right]^{\dagger}\left(A^{*} A\right)^{k-1} A^{*}\right\} \\
= & r\left[\begin{array}{c}
M^{\dagger} \\
\left.B^{*}\left(B B^{*}\right)^{k-1}\left[\left(A^{*} A\right)^{k}\left(B B^{*}\right)^{k}\right]^{\dagger}\left(A^{*} A\right)^{k-1} A^{*}\right]
\end{array}\right. \\
& +r\left[M^{\dagger}, B^{*}\left(B B^{*}\right)^{k-1}\left[\left(A^{*} A\right)^{k}\left(B B^{*}\right)^{k}\right]^{\dagger}\left(A^{*} A\right)^{k-1} A^{*}\right] \\
& -r\left(M^{\dagger}\right)-r\left\{B^{*}\left(B B^{*}\right)^{k-1}\left[\left(A^{*} A\right)^{k}\left(B B^{*}\right)^{k}\right]^{\dagger}\left(A^{*} A\right)^{k-1} A^{*}\right\} \\
= & r\left[\begin{array}{c}
M^{*} \\
B^{*}\left(A^{*} A\right)^{2 k-1} A^{*}
\end{array}\right]+r\left[M^{*}, B^{*}\left(B B^{*}\right)^{2 k-1} A^{*}\right]-2 r(M) \\
= & r\left[\begin{array}{c}
M \\
A\left(B B^{*}\right)^{2 k-1} B
\end{array}\right]+r\left[M, A\left(A^{*} A\right)^{2 k-1} B\right]-2 r(M) .
\end{aligned}
$$

So that

$$
\begin{aligned}
& M^{\dagger}=B^{*}\left(B B^{*}\right)^{k-1}\left[\left(A^{*} A\right)^{k}\left(B B^{*}\right)^{k}\right]^{\dagger}\left(A^{*} A\right)^{k-1} A^{*} \\
& \Leftrightarrow \mathscr{R}\left[A\left(A^{*} A\right)^{2 k-1} B\right]=\mathscr{R}(M) \text { and } \mathscr{R}\left[B^{*}\left(B B^{*}\right)^{2 k-1} A^{*}\right]=\mathscr{R}\left(M^{*}\right) .
\end{aligned}
$$

Applying the equivalence of $\langle 1\rangle$ and $\langle 65\rangle$ to the product $\left(A^{*} A\right)^{k}\left(B B^{*}\right)^{k}$ yields

$$
\left[\left(A^{*} A\right)^{k}\left(B B^{*}\right)^{k}\right]^{\dagger}=\left[\left(B B^{*}\right)^{k}\right]^{\dagger}\left[\left(A^{*} A\right)^{k}\right]^{\dagger} \Leftrightarrow \mathscr{R}\left[\left(A^{*} A\right)^{2 k} B\right] \subseteq \mathscr{R}(B) \text { and } \mathscr{R}\left[\left(B B^{*}\right)^{2 k} A^{*}\right] \subseteq \mathscr{R}\left(A^{*}\right) .
$$

Also note from (11.22) that

$$
\begin{aligned}
& \mathscr{R}\left(A^{*} M\right) \subseteq \mathscr{R}(B) \Rightarrow \mathscr{R}\left[\left(A^{*} A\right)^{2 k} B\right] \subseteq \mathscr{R}(B) \text { and } \mathscr{R}\left[A\left(A^{*} A\right)^{2 k-1} B\right]=\mathscr{R}(M), \\
& \mathscr{R}\left(B M^{*}\right) \subseteq \mathscr{R}\left(A^{*}\right) \Rightarrow \mathscr{R}\left[\left(B B^{*}\right)^{2 k} A^{*}\right] \subseteq \mathscr{R}\left(A^{*}\right) \text { and } \mathscr{R}\left[B^{*}\left(B B^{*}\right)^{2 k-1} A^{*}\right]=\mathscr{R}\left(M^{*}\right) .
\end{aligned}
$$

Thus if $\langle 1\rangle$ holds, combining $\langle 65\rangle$ with $(11.26-(11.29)$, we see that the two equalities in $\langle 49\rangle$ hold.

Merging the two equalities in $\langle 50\rangle$ and simplifying yields the equality in $\langle 1\rangle$. Conversely, notice that $B^{*} B\left(A A^{*} A B B^{*} B\right)^{\dagger} A A^{*}$ is a $\{2\}$-inverses of $A B$. Then we obtain by (4.20) that

$$
\begin{aligned}
r\left[M^{\dagger}-B^{*} B\left(A A^{*} A B B^{*} B\right)^{\dagger} A A^{*}\right] & =r\left[\begin{array}{c}
M^{\dagger} \\
B^{*} B\left(A A^{*} A B B^{*} B\right)^{\dagger} A A^{*}
\end{array}\right]+r\left[M^{\dagger}, B^{*} B\left(A A^{*} A B B^{*} B\right)^{\dagger} A A^{*}\right] \\
& -r\left(M^{\dagger}\right)-r\left[B^{*} B\left(A A^{*} A B B^{*} B\right)^{\dagger} A A^{*}\right] \\
& =r\left[\begin{array}{c}
M^{*} \\
B^{*} A^{*}\left(A A^{*}\right)^{2}
\end{array}\right]+r\left[M^{*},\left(B^{*} B\right)^{2} B^{*} A^{*}\right]-2 r(M) \\
& =r\left[\begin{array}{c}
M \\
M\left(B^{*} B\right)^{2}
\end{array}\right]+r\left[M,\left(A A^{*}\right)^{2} M\right]-2 r(M)
\end{aligned}
$$


So that

$$
M^{\dagger}=B^{*} B\left(A A^{*} A B B^{*} B\right)^{\dagger} A A^{*} \Leftrightarrow \mathscr{R}\left[\left(A A^{*}\right)^{2} M\right]=\mathscr{R}(M) \text { and } \mathscr{R}\left[\left(B^{*} B\right)^{2} M^{*}\right]=\mathscr{R}\left(M^{*}\right) .
$$

Applying the equivalence of $\langle 1\rangle$ and $\langle 65\rangle$ to the product $A A^{*} A B B^{*} B$ yields

$$
\left(A A^{*} A B B^{*} B\right)^{\dagger}=\left(B B^{*} B\right)^{\dagger}\left(A A^{*} A\right)^{\dagger} \Leftrightarrow \mathscr{R}\left[\left(A^{*} A\right)^{3} B\right] \subseteq \mathscr{R}(B) \text { and } \mathscr{R}\left[\left(B B^{*}\right)^{3} A^{*}\right] \subseteq \mathscr{R}\left(A^{*}\right) .
$$

Also by (8.19),

$$
\begin{aligned}
& \mathscr{R}\left(A^{*} M\right) \subseteq \mathscr{R}(B) \Rightarrow \mathscr{R}\left[\left(A^{*} A\right)^{3} B\right] \subseteq \mathscr{R}(B) \text { and } \mathscr{R}\left[\left(A A^{*}\right)^{2} M\right]=\mathscr{R}(M), \\
& \left.\mathscr{R}\left(B M^{*}\right) \subseteq \mathscr{R}\left(A^{*}\right) \Rightarrow \mathscr{R}\left[\left(B B^{*}\right)^{3} A^{*}\right] \subseteq \mathscr{R}\left(A^{*}\right) \text { and } \mathscr{R}\left[\left(B^{*} B\right)^{2} M^{*}\right)\right]=\mathscr{R}\left(M^{*}\right) .
\end{aligned}
$$

Thus if $\langle 1\rangle$ holds, combining $\langle 65\rangle$ with 11.31 - 11.34), we see that the two equalities in $\langle 50\rangle$ hold.

The equivalence of $\langle 51\rangle$ and $\langle 65\rangle$ follows from Theorem 9.4(a) $\langle 9\rangle$ and $\langle 26\rangle$ and Theorem 9.5(a) $\langle 9\rangle$ and $\langle 26\rangle$.

The equivalence of $\langle 52\rangle$ and $\langle 65\rangle$ follows from Theorem 9.4(a) $\langle 23\rangle$ and $\langle 26\rangle$ and Theorem 9.5(a) $\langle 23\rangle$ and $\langle 26\rangle$.

The equivalence of $\langle 53\rangle$ and $\langle 65\rangle$ follows from Theorem 9.4(a) $\langle 23\rangle$ and $\langle 26\rangle$ and Theorem 9.5(a) $\langle 23\rangle$ and $\langle 26\rangle$.

The equivalence of $\langle 54\rangle$ and $\langle 65\rangle$ follows from Theorem 9.4(a) $\langle 13\rangle$ and $\langle 26\rangle$ and Theorem 9.5(a) $\langle 13\rangle$ and $\langle 26\rangle$.

The equivalence of $\langle 55\rangle$ and $\langle 65\rangle$ follows from Theorem 9.4(a) $\langle 14\rangle$ and $\langle 26\rangle$ and Theorem 9.5(a) $\langle 14\rangle$ and $\langle 26\rangle$.

The equivalence of $\langle 56\rangle$ and $\langle 68\rangle$ follows from Theorem 9.1(a) $\langle 87\rangle$ and $\langle 116\rangle$, Theorem $11.1\langle 2\rangle$ and $\langle 9\rangle$, and

Theorem $11.2\langle 2\rangle$ and $\langle 9\rangle$.

The equivalence of $\langle 57\rangle$ and $\langle 68\rangle$ follows from Theorem 9.1(a) $\langle 87\rangle$ and $\langle 116\rangle$, Theorem 11.1 $\langle 3\rangle$ and $\langle 9\rangle$, and Theorem $11.2\langle 3\rangle$ and $\langle 9\rangle$.

The equivalence of $\langle 58\rangle$ and $\langle 68\rangle$ follows from Theorem 9.1(a) $\langle 87\rangle$ and $\langle 116\rangle$, Theorem $11.1\langle 4\rangle$ and $\langle 9\rangle$, and Theorem $11.2\langle 4\rangle$ and $\langle 9\rangle$.

The equivalence of $\langle 59\rangle$ and $\langle 68\rangle$ follows from Theorem 9.1(a) $\langle 87\rangle$ and $\langle 116\rangle$, Theorem $11.1\langle 5\rangle$ and $\langle 9\rangle$, and Theorem $11.2\langle 5\rangle$ and $\langle 9\rangle$.

The equivalence of $\langle 60\rangle$ and $\langle 68\rangle$ follows from Theorem 9.1(a) $\langle 87\rangle$ and $\langle 116\rangle$, Theorem 11.1 $\langle 6\rangle$ and $\langle 9\rangle$, and Theorem $11.2\langle 6\rangle$ and $\langle 9\rangle$.

The equivalence of $\langle 61\rangle$ and $\langle 68\rangle$ follows from Theorem 9.4(a) $\langle 21\rangle$ and $\langle 26\rangle$, and Theorem 9.5 21$\rangle$ and $\langle 26\rangle$.

The equivalence of $\langle 62\rangle$ and $\langle 68\rangle$ follows from Theorem 9.1(a) $\langle 116\rangle$ and $\langle 155\rangle$, Theorem $11.1\langle 8\rangle$ and $\langle 9\rangle$, and Theorem $11.2\langle 8\rangle$ and $\langle 9\rangle$.

It can be derived from (4.10) that

$$
\begin{aligned}
& r\left[A^{\dagger} A B B^{\dagger}, B B^{*} A^{*} A\right]=r\left[B M^{*}, A^{*}\right]+r(M)-r(A), \\
& r\left[B B^{\dagger} A^{\dagger} A, A^{*} A B B^{*}\right]=r\left[A^{*} M, B\right]+r(M)-r(B) .
\end{aligned}
$$

Thus

$$
\begin{aligned}
& \mathscr{R}\left(A^{\dagger} A B B^{\dagger}\right)=\mathscr{R}\left(B B^{*} A^{*} A\right) \Leftrightarrow r\left[B M^{*}, A^{*}\right]=r(A), \\
& \mathscr{R}\left(B B^{\dagger} A^{\dagger} A\right)=\mathscr{R}\left(A^{*} A B B^{*}\right) \Leftrightarrow r\left[A^{*} M, B\right]=r(B) .
\end{aligned}
$$

If $\langle 1\rangle$ holds, then $\langle 63\rangle$ implies $\langle 64\rangle$ by (11.37), 11.38), and Theorem 9.1(a) $\langle 1\rangle$ and $\langle 155\rangle$. Conversely, $\langle 64\rangle$ obviously implies $\langle 65\rangle$, and thus it implies $\langle 1\rangle$ as well.

Applying (4.10) to $M^{*}-B^{*} B M^{\dagger} A A^{*}$ gives

$$
r\left(M^{*}-B^{*} B M^{\dagger} A A^{*}\right)=r\left[\begin{array}{cc}
M^{*} M M^{*} & M^{*} A^{*} \\
B^{*} M^{*} & B^{*} A^{*}
\end{array}\right]-r(M)=r\left[\begin{array}{cc}
M M^{*} M & M B^{*} B \\
A A^{*} M & M
\end{array}\right]-r(M) .
$$

Setting both sides of 11.39 ) equal to zero leads to the equivalence of $\langle 39\rangle$ yields $\langle 69\rangle$.

The equivalence of $\langle 69\rangle$ and $\langle 70\rangle$ are derived from Lemma $4.2(\mathrm{e})$.

The equivalence of $\langle 69\rangle$ and $\langle 71\rangle$ are derived from $r(J)=t_{1}=t_{2}=r(M)$ in 4.30 .

The equivalence of $\langle 69\rangle$ and $\langle 72\rangle$ are derived from $r(J)=t_{3}=t_{4}=r(M)$ in 4.31 .

The equivalence of $\langle 65\rangle$ and $\langle 73\rangle$ are derived from Lemma 5.2(b) and (e).

Some equivalent statements in Theorem 11.3 were formulated by different authors and were scattered in the literature. But we prefer to giving complete proofs for the equivalences of all these statements in order to sufficiently recognize and use this collection of results in different situations.

Many subsequent results can be established on ROLs for the Moore-Penrose inverses of matrix products by the replacements of $A$ and $B$ with $\left(A^{\dagger}\right)^{*}$ and $\left(B^{\dagger}\right)^{*}$, as well as $\left(A^{*} A\right)^{\frac{1}{2}}$ and $\left(B B^{*}\right)^{\frac{1}{2}}$, respectively.

Corollary 11.4. Let $A \in \mathbb{C}^{m \times n}$ and $B \in \mathbb{C}^{n \times p}$, and denote $\widehat{A}=\left(A^{\dagger}\right)^{*}$ and $M=\left(A^{\dagger}\right)^{*} B$. Then the following 74 statements are equivalent: 
$\langle 0\rangle(A B)^{\dagger}=B^{\dagger} A^{\dagger}$.

$\langle 2\rangle M^{\dagger} \in\left\{B^{\dagger} \widehat{A}^{(1,3,4)}\right\}$.

〈4) $M^{\dagger} \in\left\{B^{\dagger} \widehat{A}^{(1,4)}\right\}$.

$\langle 6\rangle M^{\dagger} \in\left\{B^{(1,3,4)} \widehat{A}^{(1,3,4)}\right\}$.

$\langle 8\rangle M^{\dagger} \in\left\{B^{(1,3,4)} \widehat{A}^{(1,4)}\right\}$.

$\langle 10\rangle M^{\dagger} \in\left\{B^{(1,2,3)} \widehat{A}^{(1,3,4)}\right\}$.

$\langle 12\rangle M^{\dagger} \in\left\{B^{(1,2,3)} \widehat{A}^{(1,4)}\right\}$.

$\langle 14\rangle M^{\dagger} \in\left\{B^{(1,3)} \widehat{A}^{(1,3,4)}\right\}$.

$\langle 16\rangle M^{\dagger} \in\left\{B^{(1,3)} \widehat{A}^{(1,4)}\right\}$.

$\langle 18\rangle\left\{M^{(1,3,4)}\right\} \supseteq\left\{B^{(1,3,4)} \widehat{A}^{\dagger}\right\}$.

$\langle 20\rangle$ Both $\left\{M^{(1,3)}\right\} \ni B^{\dagger} \widehat{A}^{\dagger}$ and $\left\{M^{(1,4)}\right\} \ni B^{\dagger} \widehat{A}^{\dagger}$.

$\langle 21\rangle$ Both $\left\{M^{(1,3)}\right\} \supseteq\left\{B^{(1,3,4)} \widehat{A}^{\dagger}\right\}$ and $\left\{M^{(1,4)}\right\} \supseteq\left\{B^{\dagger} \widehat{A}^{(1,3,4)}\right\}$.

$\langle 22\rangle$ Both $\left\{M^{(1,3)}\right\} \supseteq\left\{B^{(1,2,3)} \widehat{A}^{\dagger}\right\}$ and $\left\{M^{(1,4)}\right\} \supseteq\left\{B^{\dagger} \widehat{A}^{(1,2,4)}\right\}$.

$\langle 23\rangle$ Both $\left\{M^{(1,3)}\right\} \supseteq\left\{B^{(1,3)} \widehat{A}^{\dagger}\right\}$ and $\left\{M^{(1,4)}\right\} \supseteq\left\{B^{\dagger} \widehat{A}^{(1,4)}\right\}$.

$\langle 24\rangle$ Both $\left\{M^{(1,3)}\right\} \supseteq\left\{B^{\dagger} \widehat{A}^{(1,3,4)}\right\}$ and $\left\{M^{(1,4)}\right\} \supseteq\left\{B^{(1,3,4)} \widehat{A}^{\dagger}\right\}$.

$\langle 25\rangle$ Both $\left\{M^{(1,3)}\right\} \supseteq\left\{B^{(1,3,4)} \widehat{A}^{(1,3,4)}\right\}$ and $\left\{M^{(1,4)}\right\} \supseteq\left\{B^{(1,3,4)} \widehat{A}^{(1,3,4)}\right\}$.

$\langle 26\rangle$ Both $\left\{M^{(1,3)}\right\} \supseteq\left\{B^{(1,2,3)} \widehat{A}^{(1,3,4)}\right\}$ and $\left\{M^{(1,4)}\right\} \supseteq\left\{B^{(1,3,4)} \widehat{A}^{(1,2,4)}\right\}$.

$\langle 27\rangle$ Both $\left\{M^{(1,3)}\right\} \supseteq\left\{B^{(1,3)} \widehat{A}^{(1,3,4)}\right\}$ and $\left\{M^{(1,4)}\right\} \supseteq\left\{B^{(1,3,4)} \widehat{A}^{(1,3)}\right\}$.

$\langle 28\rangle$ Both $\left\{M^{(1,3)}\right\} \supseteq\left\{B^{\dagger} \widehat{A}^{(1,2,3)}\right\}$ and $\left\{M^{(1,4)}\right\} \supseteq\left\{B^{(1,2,4)} \widehat{A}^{\dagger}\right\}$.

$\langle 29\rangle$ Both $\left\{M^{(1,3)}\right\} \supseteq\left\{B^{(1,3,4)} \widehat{A}^{(1,2,3)}\right\}$ and $\left\{M^{(1,4)}\right\} \supseteq\left\{B^{(1,2,4)} \widehat{A}^{(1,3,34)}\right\}$.

$\langle 30\rangle$ Both $\left\{M^{(1,3)}\right\} \supseteq\left\{B^{(1,2,3)} \widehat{A}^{(1,2,3)}\right\}$ and $\left\{M^{(1,4)}\right\} \supseteq\left\{B^{(1,2,4)} \widehat{A}^{(1,2,4)}\right\}$.

$\langle 31\rangle$ Both $\left\{M^{(1,3)}\right\} \supseteq\left\{B^{(1,3)} \widehat{A}^{(1,2,3)}\right\}$ and $\left\{M^{(1,4)}\right\} \supseteq\left\{B^{(1,2,4)} \widehat{A}^{(1,4)}\right\}$.

$\langle 32\rangle$ Both $\left\{M^{(1,3)}\right\} \supseteq\left\{B^{\dagger} \widehat{A}^{(1,3)}\right\}$ and $\left\{M^{(1,4)}\right\} \supseteq\left\{B^{(1,4)} \widehat{A}^{\dagger}\right\}$.

$\langle 33\rangle$ Both $\left\{M^{(1,3)}\right\} \supseteq\left\{B^{(1,3,4)} \widehat{A}^{(1,3)}\right\}$ and $\left\{M^{(1,4)}\right\} \supseteq\left\{B^{(1,4)} \widehat{A}^{(1,3,4)}\right\}$.

$\langle 34\rangle$ Both $\left\{M^{(1,3)}\right\} \supseteq\left\{B^{(1,2,3)} \widehat{A}^{(1,3)}\right\}$ and $\left\{M^{(1,4)}\right\} \supseteq\left\{B^{(1,4)} \widehat{A}^{(1,2,4)}\right\}$.

$\langle 35\rangle$ Both $\left\{M^{(1,3)}\right\} \supseteq\left\{B^{(1,3)} \widehat{A}^{(1,3)}\right\}$ and $\left\{M^{(1,4)}\right\} \supseteq\left\{B^{(1,4)} \widehat{A}^{(1,4)}\right\}$.

$\langle 36\rangle\left\{\left(\widehat{A}^{*} M\right)^{(1)}\right\} \ni B^{\dagger}\left(\widehat{A}^{*} \widehat{A}\right)^{\dagger},\left\{\left(\widehat{A}^{*} M\right)^{(1,3)}\right\} \ni M^{\dagger}\left(\widehat{A}^{*}\right)^{\dagger},\left\{\left(M B^{*}\right)^{(1)}\right\} \ni\left(B B^{*}\right)^{\dagger} \widehat{A}^{\dagger}$, and $\left\{\left(M B^{*}\right)^{(1,4)}\right\} \ni$ $\left(B^{*}\right)^{\dagger} M^{\dagger}$.

$\langle 37\rangle M^{\dagger}=B^{\dagger} \widehat{A}^{\dagger} M B^{\dagger} \widehat{A}^{\dagger}$.

$\langle 38\rangle B M^{\dagger} \widehat{A}=B B^{\dagger} \widehat{A}^{\dagger} \widehat{A}$.

$\langle 39\rangle B^{*} B M^{\dagger} \widehat{A} \widehat{A}^{*}=M^{*}$.

$\langle 40\rangle$ Both $M M^{\dagger}=M B^{\dagger} \widehat{A}^{\dagger}$ and $M^{\dagger} M=B^{\dagger} \widehat{A}^{\dagger} M$. 
$\langle 41\rangle$ Both $M M^{\dagger} \widehat{A}=M B^{\dagger}$ and $\widehat{A}^{\dagger} M=B M^{\dagger} M$.

$\langle 42\rangle$ Both $B B^{\dagger} \widehat{A^{*}} \widehat{A} B=\widehat{A}^{*} \widehat{A} B$ and $\widehat{A} B B^{*} \widehat{A}^{\dagger} \widehat{A}=\widehat{A} B B^{*}$.

$\langle 43\rangle B B^{\dagger} \widehat{A}^{*} \widehat{A} B B^{*} \widehat{A}^{\dagger} \widehat{A}=B B^{\dagger} \widehat{A}^{*} \widehat{A} B B^{*}=\widehat{A}^{*} \widehat{A} B B^{*} \widehat{A}^{\dagger} \widehat{A}=\widehat{A}^{*} \widehat{A} B B^{*}$.

〈44〉Both $M^{\dagger}=\left(\widehat{A}^{\dagger} \widehat{A} B\right)^{\dagger} \widehat{A}^{\dagger}$ and $\left(\widehat{A}^{\dagger} \widehat{A} B\right)^{\dagger}=B^{\dagger} \widehat{A}^{\dagger} \widehat{A}$.

$\langle 45\rangle$ Both $M^{\dagger}=B^{\dagger}\left(\widehat{A} B B^{\dagger}\right)^{\dagger}$ and $\left(\widehat{A} B B^{\dagger}\right)^{\dagger}=B B^{\dagger} \widehat{A}^{\dagger}$.

$\langle 46\rangle$ Both $M^{\dagger}=\left(\widehat{A}^{*} \widehat{A} B\right)^{\dagger} \widehat{A}^{*}$ and $\left(\widehat{A}^{*} \widehat{A} B\right)^{\dagger}=B^{\dagger}\left(\widehat{A}^{*} \widehat{A}\right)^{\dagger}$.

$\langle 47\rangle$ Both $M^{\dagger}=B^{*}\left(\widehat{A} B B^{*}\right)^{\dagger}$ and $\left(\widehat{A} B B^{*}\right)^{\dagger}=\left(B B^{*}\right)^{\dagger} \widehat{A}^{\dagger}$.

$\langle 48\rangle$ Both $M^{\dagger}=B^{\dagger}\left(\widehat{A}^{\dagger} \widehat{A} B B^{\dagger}\right)^{\dagger} \widehat{A}^{\dagger}$ and $\left(\widehat{A}^{\dagger} \widehat{A} B B^{\dagger}\right)^{\dagger}=B B^{\dagger} \widehat{A}^{\dagger} \widehat{A}$.

$\langle 49\rangle$ Both $M^{\dagger}=B^{*}\left(B B^{*}\right)^{k-1}\left[\left(\widehat{A}^{*} \widehat{A}\right)^{k}\left(B B^{*}\right)^{k}\right]^{\dagger}\left(\widehat{A}^{*} \widehat{A}\right)^{k-1} \widehat{A}^{*}$ and $\left[\left(\widehat{A}^{*} \widehat{A}\right)^{k}\left(B B^{*}\right)^{k}\right]^{\dagger}=\left[\left(B B^{*}\right)^{k}\right]^{\dagger}\left[\left(\widehat{A}^{*} \widehat{A}\right)^{k}\right]^{\dagger}$ for any integer $k \geq 1$.

$\langle 50\rangle$ Both $M^{\dagger}=B^{*} B\left(\widehat{A} \widehat{A}^{*} \widehat{A} B B^{*} B\right)^{\dagger} \widehat{A} \widehat{A}^{*}$ and $\left(\widehat{A} \widehat{A}^{*} \widehat{A} B B^{*} B\right)^{\dagger}=\left(B B^{*} B\right)^{\dagger}\left(\widehat{A} \widehat{A}^{*} \widehat{A}\right)^{\dagger}$.

$\langle 51\rangle \operatorname{Both}\left(\widehat{A} B B^{\dagger}\right)^{\dagger}=B B^{\dagger} \widehat{A}^{\dagger}$ and $\left(\widehat{A}^{\dagger} \widehat{A} B\right)^{\dagger}=B^{\dagger} \widehat{A}^{\dagger} \widehat{A}$.

$\langle 52\rangle$ Both $M B^{\dagger} \widehat{A}^{\dagger}$ and $B^{\dagger} \widehat{A}^{\dagger} M$ are orthogonal projectors.

$\langle 53\rangle$ Both $\widehat{A} E_{B} \widehat{A}^{\dagger}$ and $B^{\dagger} F_{\widehat{A}} B$ are orthogonal projectors.

$\langle 54\rangle$ Both $M M^{\dagger} \widehat{A}=M B^{\dagger}$ and $B M^{\dagger} M=\widehat{A}^{\dagger} M$.

$\langle 55\rangle$ Both $\widehat{A}^{*} \widehat{A} B B^{\dagger}=B B^{\dagger} \widehat{A}^{*} \widehat{A}$ and $\widehat{A}^{\dagger} \widehat{A} B B^{*}=B B^{*} \widehat{A}^{\dagger} \widehat{A}$.

$\langle 56\rangle$ Both $M B^{\dagger} \widehat{A}^{\dagger} M=M$ and $M^{\dagger}=\left(\widehat{A}^{\dagger} M\right)^{\dagger} \widehat{A}^{\dagger}=B^{\dagger}\left(M B^{\dagger}\right)^{\dagger}$.

$\langle 57\rangle$ Both $M B^{\dagger} \widehat{A}^{\dagger} M=M$ and $M^{\dagger}=\left(\widehat{A}^{*} M\right)^{\dagger} \widehat{A}^{*}=B^{*}\left(M B^{*}\right)^{\dagger}$.

$\langle 58\rangle M B^{\dagger} \widehat{A}^{\dagger} M=M,\left(\widehat{A}^{\dagger} M\right)^{\dagger}=M^{\dagger} \widehat{A}$, and $\left(M B^{\dagger}\right)^{\dagger}=B M^{\dagger}$.

$\langle 59\rangle M B^{\dagger} \widehat{A}^{\dagger} M=M,\left(\widehat{A}^{\dagger} M M^{\dagger} \widehat{A}\right)^{*}=\widehat{A}^{\dagger} M M^{\dagger} \widehat{A}$, and $\left(B M^{\dagger} M B^{\dagger}\right)^{*}=B M^{\dagger} M B^{\dagger}$.

$\langle 60\rangle M B^{\dagger} \widehat{A}^{\dagger} M=M, M M^{\dagger} \widehat{A} \widehat{A}^{*}=\widehat{A} \widehat{A}^{*} M M^{\dagger}$, and $M^{\dagger} M B^{*} B=B^{*} B M^{\dagger} M$.

$\langle 61\rangle$ Both $\mathscr{R}\left(\widehat{A}^{*} \widehat{A} B B^{\dagger}\right)=\mathscr{R}\left(B B^{\dagger} \widehat{A}^{*} \widehat{A}\right)$ and $\mathscr{R}\left(\widehat{A}^{\dagger} \widehat{A} B B^{*}\right)=\mathscr{R}\left(B B^{*} \widehat{A}^{\dagger} \widehat{A}\right)$.

$\langle 62\rangle \mathscr{R}\left(\widehat{A}^{\dagger} \widehat{A} B B^{\dagger}\right)=\mathscr{R}\left(B B^{\dagger} \widehat{A}^{\dagger} \widehat{A}\right), \mathscr{R}\left(M^{\dagger}\right)=\mathscr{R}\left(B^{\dagger} \widehat{A}^{\dagger}\right)$, and $\mathscr{R}\left[\left(M^{*}\right)^{\dagger}\right]=\mathscr{R}\left[\left(\widehat{A}^{*}\right)^{\dagger}\left(B^{*}\right)^{\dagger}\right]$.

$\langle 63\rangle$ Both $\mathscr{R}\left(\widehat{A}^{\dagger} \widehat{A} B B^{\dagger}\right)=\mathscr{R}\left(B B^{*} \widehat{A}^{*} \widehat{A}\right)$ and $\mathscr{R}\left(B B^{\dagger} \widehat{A}^{\dagger} \widehat{A}\right)=\mathscr{R}\left(\widehat{A}^{*} \widehat{A} B B^{*}\right)$.

$\langle 64\rangle \mathscr{R}\left(\widehat{A}^{*} \widehat{A} B B^{*}\right)=\mathscr{R}\left(B B^{*} \widehat{A}^{*} \widehat{A}\right)$.

$\langle 65\rangle$ Both $\mathscr{R}\left(\widehat{A}^{*} M\right) \subseteq \mathscr{R}(B)$ and $\mathscr{R}\left(B M^{*}\right) \subseteq \mathscr{R}\left(\widehat{A}^{*}\right)$.

$\langle 66\rangle$ Both $\mathscr{R}\left(\widehat{A}^{*} M\right)=\mathscr{R}\left(\widehat{A}^{*}\right) \cap \mathscr{R}(B)$ and $\mathscr{R}\left(B M^{*}\right)=\mathscr{R}(B) \cap \mathscr{R}\left(\widehat{A}^{*}\right)$.

$\langle 67\rangle$ Both $r\left[\widehat{A}^{*} M, B\right]=r(B)$ and $r\left[B M^{*}, \widehat{A}^{*}\right]=r(\widehat{A})$.

$\langle 68\rangle r\left[\widehat{A}^{*}, B\right]=r(\widehat{A})+r(B)-r(M), r\left[\widehat{A} \widehat{A}^{*} M, M\right]=r(M)$, and $r\left[B^{*} B M^{*}, M^{*}\right]=r(M)$.

$\langle 69\rangle r\left[\begin{array}{cc}M M^{*} M & M B^{*} B \\ \widehat{A} \widehat{A}^{*} M & M\end{array}\right]=r(M)$

$\langle 70\rangle M B^{*} B M^{\dagger} \widehat{A} \widehat{A}^{*} M=M M^{*} M, r\left[\widehat{A} \widehat{A}^{*} M, M\right]=r(M)$, and $r\left[B^{*} B M^{*}, M^{*}\right]=r(M)$.

$\langle 71\rangle r\left[\begin{array}{cc}M M^{*} M & M B^{*} B \\ \widehat{A} \widehat{A}^{*} M & M\end{array}\right]=r\left[\widehat{A}^{*}, B\right]+2 r(M)-r(\widehat{A})-r(B)$ and $r\left[\widehat{A}^{*} M, B\right]+r\left[B M^{*}, \widehat{A}^{*}\right]=r\left[\widehat{A}^{*}, B\right]+r(M)$.

$\langle 72\rangle r\left[\begin{array}{cc}M M^{*} M & M B^{*} B \\ \widehat{A} \widehat{A}^{*} M & M\end{array}\right]=r\left[\widehat{A}^{*} M, B\right]+r\left[B M^{*}, \widehat{A}^{*}\right]+r(\widehat{A})+r(B)-2 r\left[\widehat{A}^{*}, B\right]-r(\widehat{A} B), r\left[\widehat{A} \widehat{A}^{*} M, M\right]=r(M)$, and $r\left[B^{*} B M^{*}, M^{*}\right]=r(M)$. 
$\langle 73\rangle$ The matrix equation $B X \widehat{A}=\widehat{A}^{*} \widehat{A} B B^{*}$ is consistent.

Corollary 11.5. Let $A \in \mathbb{C}^{m \times n}$ and $B \in \mathbb{C}^{n \times p}$, and denote $\widehat{B}=\left(B^{\dagger}\right)^{*}$ and $M=A\left(B^{\dagger}\right)^{*}$. Then the following 74 statements are equivalent:
$\langle 0\rangle(A B)^{\dagger}=B^{\dagger} A^{\dagger}$
$\langle 1\rangle M^{\dagger}=\widehat{B}^{\dagger} A^{\dagger}$
$\langle 2\rangle M^{\dagger} \in\left\{\widehat{B}^{\dagger} A^{(1,3,4)}\right\}$.
$\langle 3\rangle M^{\dagger} \in\left\{\widehat{B}^{\dagger} A^{(1,2,4)}\right\}$.
〈4〉 $M^{\dagger} \in\left\{\widehat{B}^{\dagger} A^{(1,4)}\right\}$.
$\langle 5\rangle M^{\dagger} \in\left\{\widehat{B}^{(1,3,4)} A^{\dagger}\right\}$.
$\langle 6\rangle M^{\dagger} \in\left\{\widehat{B}^{(1,3,4)} A^{(1,3,4)}\right\}$.
$\langle 7\rangle M^{\dagger} \in\left\{\widehat{B}^{(1,3,4)} A^{(1,2,4)}\right\}$.
$\langle 8\rangle M^{\dagger} \in\left\{\widehat{B}^{(1,3,4)} A^{(1,4)}\right\}$.
$\langle 9\rangle M^{\dagger} \in\left\{\widehat{B}^{(1,2,3)} A^{\dagger}\right\}$.
$\langle 10\rangle M^{\dagger} \in\left\{\widehat{B}^{(1,2,3)} A^{(1,3,4)}\right\}$.
$\langle 11\rangle M^{\dagger} \in\left\{\widehat{B}^{(1,2,3)} A^{(1,2,4)}\right\}$.
$\langle 12\rangle M^{\dagger} \in\left\{\widehat{B}^{(1,2,3)} A^{(1,4)}\right\}$.
$\langle 13\rangle M^{\dagger} \in\left\{\widehat{B}^{(1,3)} A^{\dagger}\right\}$.
$\langle 14\rangle M^{\dagger} \in\left\{\widehat{B}^{(1,3)} A^{(1,3,4)}\right\}$.
115〉 $M^{\dagger} \in\left\{\widehat{B}^{(1,3)} A^{(1,2,4)}\right\}$.
$\langle 16\rangle M^{\dagger} \in\left\{\widehat{B}^{(1,3)} A^{(1,4)}\right\}$.
$\langle 17\rangle\left\{M^{(1,3,4)}\right\} \supseteq\left\{\widehat{B}^{\dagger} A^{(1,3,4)}\right\}$.
$\langle 18\rangle\left\{M^{(1,3,4)}\right\} \supseteq\left\{\widehat{B}^{(1,3,4)} A^{\dagger}\right\}$.
$\langle 19\rangle\left\{M^{(1,3,4)}\right\} \supseteq\left\{\widehat{B}^{(1,3,4)} A^{(1,3,4)}\right\}$.

$\langle 20\rangle$ Both $\left\{M^{(1,3)}\right\} \ni \widehat{B}^{\dagger} A^{\dagger}$ and $\left\{M^{(1,4)}\right\} \ni \widehat{B}^{\dagger} A^{\dagger}$.

$\langle 21\rangle \operatorname{Both}\left\{M^{(1,3)}\right\} \supseteq\left\{\widehat{B}^{(1,3,4)} A^{\dagger}\right\}$ and $\left\{M^{(1,4)}\right\} \supseteq\left\{\widehat{B}^{\dagger} A^{(1,3,4)}\right\}$.

$\langle 22\rangle \operatorname{Both}\left\{M^{(1,3)}\right\} \supseteq\left\{\widehat{B}^{(1,2,3)} A^{\dagger}\right\}$ and $\left\{M^{(1,4)}\right\} \supseteq\left\{\widehat{B}^{\dagger} A^{(1,2,4)}\right\}$.

$\langle 23\rangle \operatorname{Both}\left\{M^{(1,3)}\right\} \supseteq\left\{\widehat{B}^{(1,3)} A^{\dagger}\right\}$ and $\left\{M^{(1,4)}\right\} \supseteq\left\{\widehat{B}^{\dagger} A^{(1,4)}\right\}$.

$\langle 24\rangle \operatorname{Both}\left\{M^{(1,3)}\right\} \supseteq\left\{\widehat{B}^{\dagger} A^{(1,3,4)}\right\}$ and $\left\{M^{(1,4)}\right\} \supseteq\left\{\widehat{B}^{(1,3,4)} A^{\dagger}\right\}$.

$\langle 25\rangle$ Both $\left\{M^{(1,3)}\right\} \supseteq\left\{\widehat{B}^{(1,3,4)} A^{(1,3,4)}\right\}$ and $\left\{M^{(1,4)}\right\} \supseteq\left\{\widehat{B}^{(1,3,4)} A^{(1,3,4)}\right\}$.

$\langle 26\rangle$ Both $\left\{M^{(1,3)}\right\} \supseteq\left\{\widehat{B}^{(1,2,3)} A^{(1,3,4)}\right\}$ and $\left\{M^{(1,4)}\right\} \supseteq\left\{\widehat{B}^{(1,3,4)} A^{(1,2,4)}\right\}$.

$\langle 27\rangle$ Both $\left\{M^{(1,3)}\right\} \supseteq\left\{\widehat{B}^{(1,3)} A^{(1,3,4)}\right\}$ and $\left\{M^{(1,4)}\right\} \supseteq\left\{\widehat{B}^{(1,3,4)} A^{(1,3)}\right\}$.

$\langle 28\rangle \operatorname{Both}\left\{M^{(1,3)}\right\} \supseteq\left\{\widehat{B}^{\dagger} A^{(1,2,3)}\right\}$ and $\left\{M^{(1,4)}\right\} \supseteq\left\{\widehat{B}^{(1,2,4)} A^{\dagger}\right\}$.

$\langle 29\rangle$ Both $\left\{M^{(1,3)}\right\} \supseteq\left\{\widehat{B}^{(1,3,4)} A^{(1,2,3)}\right\}$ and $\left\{M^{(1,4)}\right\} \supseteq\left\{\widehat{B}^{(1,2,4)} A^{(1,3,34)}\right\}$.

$\langle 30\rangle$ Both $\left\{M^{(1,3)}\right\} \supseteq\left\{\widehat{B}^{(1,2,3)} A^{(1,2,3)}\right\}$ and $\left\{M^{(1,4)}\right\} \supseteq\left\{\widehat{B}^{(1,2,4)} A^{(1,2,4)}\right\}$.

$\langle 31\rangle$ Both $\left\{M^{(1,3)}\right\} \supseteq\left\{\widehat{B}^{(1,3)} A^{(1,2,3)}\right\}$ and $\left\{M^{(1,4)}\right\} \supseteq\left\{\widehat{B}^{(1,2,4)} A^{(1,4)}\right\}$.

$\langle 32\rangle$ Both $\left\{M^{(1,3)}\right\} \supseteq\left\{\widehat{B}^{\dagger} A^{(1,3)}\right\}$ and $\left\{M^{(1,4)}\right\} \supseteq\left\{\widehat{B}^{(1,4)} A^{\dagger}\right\}$.

$\langle 33\rangle$ Both $\left\{M^{(1,3)}\right\} \supseteq\left\{\widehat{B}^{(1,3,4)} A^{(1,3)}\right\}$ and $\left\{M^{(1,4)}\right\} \supseteq\left\{\widehat{B}^{(1,4)} A^{(1,3,4)}\right\}$.

$\langle 34\rangle$ Both $\left\{M^{(1,3)}\right\} \supseteq\left\{\widehat{B}^{(1,2,3)} A^{(1,3)}\right\}$ and $\left\{M^{(1,4)}\right\} \supseteq\left\{\widehat{B}^{(1,4)} A^{(1,2,4)}\right\}$.

$\langle 35\rangle$ Both $\left\{M^{(1,3)}\right\} \supseteq\left\{\widehat{B}^{(1,3)} A^{(1,3)}\right\}$ and $\left\{M^{(1,4)}\right\} \supseteq\left\{\widehat{B}^{(1,4)} A^{(1,4)}\right\}$.

$\langle 36\rangle\left\{\left(A^{*} M\right)^{(1)}\right\} \ni \widehat{B}^{\dagger}\left(A^{*} A\right)^{\dagger},\left\{\left(A^{*} M\right)^{(1,3)}\right\} \ni M^{\dagger}\left(A^{*}\right)^{\dagger},\left\{\left(M \widehat{B}^{*}\right)^{(1)}\right\} \ni\left(\widehat{B} \widehat{B}^{*}\right)^{\dagger} A^{\dagger}$, and $\left\{\left(M \widehat{B}^{*}\right)^{(1,4)}\right\} \ni$ $\left(\widehat{B}^{*}\right)^{\dagger} M^{\dagger}$. 
$\langle 37\rangle M^{\dagger}=\widehat{B}^{\dagger} A^{\dagger} M \widehat{B}^{\dagger} A^{\dagger} . \quad\langle 38\rangle \widehat{B} M^{\dagger} A=\widehat{B} \widehat{B}^{\dagger} A^{\dagger} A . \quad\langle 39\rangle \widehat{B}^{*} \widehat{B} M^{\dagger} A A^{*}=M^{*}$.

$\langle 40\rangle$ Both $M M^{\dagger}=M \widehat{B}^{\dagger} A^{\dagger}$ and $M^{\dagger} M=\widehat{B}^{\dagger} A^{\dagger} M$.

$\langle 41\rangle$ Both $M M^{\dagger} A=M \widehat{B}^{\dagger}$ and $A^{\dagger} M=\widehat{B} M^{\dagger} M$.

$\langle 42\rangle$ Both $\widehat{B} \widehat{B}^{\dagger} A^{*} A \widehat{B}=A^{*} A \widehat{B}$ and $A \widehat{B} \widehat{B}^{*} A^{\dagger} A=A \widehat{B} \widehat{B}^{*}$.

$\langle 43\rangle \widehat{B} \widehat{B}^{\dagger} A^{*} A \widehat{B} \widehat{B}^{*} A^{\dagger} A=\widehat{B} \widehat{B}^{\dagger} A^{*} A \widehat{B} \widehat{B}^{*}=A^{*} A \widehat{B} \widehat{B}^{*} A^{\dagger} A=A^{*} A \widehat{B} \widehat{B}^{*}$.

$\langle 44\rangle$ Both $M^{\dagger}=\left(A^{\dagger} A \widehat{B}\right)^{\dagger} A^{\dagger}$ and $\left(A^{\dagger} A \widehat{B}\right)^{\dagger}=\widehat{B}^{\dagger} A^{\dagger} A$.

$\langle 45\rangle$ Both $M^{\dagger}=\widehat{B}^{\dagger}\left(A \widehat{B} \widehat{B}^{\dagger}\right)^{\dagger}$ and $\left(A \widehat{B} \widehat{B}^{\dagger}\right)^{\dagger}=\widehat{B} \widehat{B}^{\dagger} A^{\dagger}$.

$\langle 46\rangle$ Both $M^{\dagger}=\left(A^{*} A \widehat{B}\right)^{\dagger} A^{*}$ and $\left(A^{*} A \widehat{B}\right)^{\dagger}=\widehat{B}^{\dagger}\left(A^{*} A\right)^{\dagger}$.

$\langle 47\rangle$ Both $M^{\dagger}=\widehat{B}^{*}\left(A \widehat{B} \widehat{B}^{*}\right)^{\dagger}$ and $\left(A \widehat{B} \widehat{B}^{*}\right)^{\dagger}=\left(\widehat{B} \widehat{B}^{*}\right)^{\dagger} A^{\dagger}$.

$\langle 48\rangle$ Both $M^{\dagger}=\widehat{B}^{\dagger}\left(A^{\dagger} A \widehat{B} \widehat{B}^{\dagger}\right)^{\dagger} A^{\dagger}$ and $\left(A^{\dagger} A \widehat{B} \widehat{B}^{\dagger}\right)^{\dagger}=\widehat{B} \widehat{B}^{\dagger} A^{\dagger} A$.

$\langle 49\rangle$ Both $M^{\dagger}=\widehat{B}^{*}\left(\widehat{B} \widehat{B}^{*}\right)^{k-1}\left[\left(A^{*} A\right)^{k}\left(\widehat{B} \widehat{B}^{*}\right)^{k}\right]^{\dagger}\left(A^{*} A\right)^{k-1} A^{*}$ and $\left[\left(A^{*} A\right)^{k}\left(\widehat{B} \widehat{B}^{*}\right)^{k}\right]^{\dagger}=\left[\left(\widehat{B} \widehat{B}^{*}\right)^{k}\right]^{\dagger}\left[\left(A^{*} A\right)^{k}\right]^{\dagger}$ for any integer $k \geq 1$.

$\langle 50\rangle$ Both $M^{\dagger}=\widehat{B}^{*} \widehat{B}\left(A A^{*} A \widehat{B} \widehat{B}^{*} \widehat{B}\right)^{\dagger} A A^{*}$ and $\left(A A^{*} A \widehat{B} \widehat{B}^{*} \widehat{B}\right)^{\dagger}=\left(\widehat{B} \widehat{B}^{*} \widehat{B}\right)^{\dagger}\left(A A^{*} A\right)^{\dagger}$.

$\langle 51\rangle$ Both $\left(A \widehat{B} \widehat{B}^{\dagger}\right)^{\dagger}=\widehat{B} \widehat{B}^{\dagger} A^{\dagger}$ and $\left(A^{\dagger} A \widehat{B}\right)^{\dagger}=\widehat{B}^{\dagger} A^{\dagger} A$.

$\langle 52\rangle$ Both $M \widehat{B}^{\dagger} A^{\dagger}$ and $\widehat{B}^{\dagger} A^{\dagger} M$ are orthogonal projectors.

$\langle 53\rangle$ Both $A E_{\widehat{B}} A^{\dagger}$ and $\widehat{B}^{\dagger} F_{A} \widehat{B}$ are orthogonal projectors.

$\langle 54\rangle$ Both $M M^{\dagger} A=M \widehat{B}^{\dagger}$ and $\widehat{B} M^{\dagger} M=A^{\dagger} M$.

$\langle 55\rangle$ Both $A^{*} A \widehat{B} \widehat{B}^{\dagger}=\widehat{B} \widehat{B}^{\dagger} A^{*} A$ and $A^{\dagger} A \widehat{B} \widehat{B}^{*}=\widehat{B} \widehat{B}^{*} A^{\dagger} A$.

$\langle 56\rangle$ Both $M \widehat{B}^{\dagger} A^{\dagger} M=M$ and $M^{\dagger}=\left(A^{\dagger} M\right)^{\dagger} A^{\dagger}=\widehat{B}^{\dagger}\left(M \widehat{B}^{\dagger}\right)^{\dagger}$.

$\langle 57\rangle$ Both $M \widehat{B}^{\dagger} A^{\dagger} M=M$ and $M^{\dagger}=\left(A^{*} M\right)^{\dagger} A^{*}=\widehat{B}^{*}\left(M \widehat{B}^{*}\right)^{\dagger}$.

$\langle 58\rangle M \widehat{B}^{\dagger} A^{\dagger} M=M,\left(A^{\dagger} M\right)^{\dagger}=M^{\dagger} A$, and $\left(M \widehat{B}^{\dagger}\right)^{\dagger}=\widehat{B} M^{\dagger}$.

$\langle 59\rangle M \widehat{B}^{\dagger} A^{\dagger} M=M,\left(A^{\dagger} M M^{\dagger} A\right)^{*}=A^{\dagger} M M^{\dagger} A$, and $\left(\widehat{B} M^{\dagger} M \widehat{B}^{\dagger}\right)^{*}=\widehat{B} M^{\dagger} M \widehat{B}^{\dagger}$.

$\langle 60\rangle M \widehat{B}^{\dagger} A^{\dagger} M=M, M M^{\dagger} A A^{*}=A A^{*} M M^{\dagger}$, and $M^{\dagger} M \widehat{B}^{*} \widehat{B}=\widehat{B}^{*} \widehat{B} M^{\dagger} M$.

$\langle 61\rangle$ Both $\mathscr{R}\left(A^{*} A \widehat{B} \widehat{B}^{\dagger}\right)=\mathscr{R}\left(\widehat{B} \widehat{B}^{\dagger} A^{*} A\right)$ and $\mathscr{R}\left(A^{\dagger} A \widehat{B} \widehat{B}^{*}\right)=\mathscr{R}\left(\widehat{B} \widehat{B}^{*} A^{\dagger} A\right)$.

$\langle 62\rangle \mathscr{R}\left(A^{\dagger} A \widehat{B} \widehat{B}^{\dagger}\right)=\mathscr{R}\left(\widehat{B} \widehat{B}^{\dagger} A^{\dagger} A\right), \mathscr{R}\left(M^{\dagger}\right)=\mathscr{R}\left(\widehat{B}^{\dagger} A^{\dagger}\right)$, and $\mathscr{R}\left[\left(M^{*}\right)^{\dagger}\right]=\mathscr{R}\left[\left(A^{*}\right)^{\dagger}\left(\widehat{B}^{*}\right)^{\dagger}\right]$.

$\langle 63\rangle$ Both $\mathscr{R}\left(A^{\dagger} A \widehat{B} \widehat{B}^{\dagger}\right)=\mathscr{R}\left(\widehat{B} \widehat{B}^{*} A^{*} A\right)$ and $\mathscr{R}\left(\widehat{B} \widehat{B}^{\dagger} A^{\dagger} A\right)=\mathscr{R}\left(A^{*} A \widehat{B} \widehat{B}^{*}\right)$.

$\langle 64\rangle \mathscr{R}\left(A^{*} A \widehat{B} \widehat{B}^{*}\right)=\mathscr{R}\left(\widehat{B} \widehat{B}^{*} A^{*} A\right)$.

$\langle 65\rangle$ Both $\mathscr{R}\left(A^{*} M\right) \subseteq \mathscr{R}(\widehat{B})$ and $\mathscr{R}\left(\widehat{B} M^{*}\right) \subseteq \mathscr{R}\left(A^{*}\right)$.

$\langle 66\rangle$ Both $\mathscr{R}\left(A^{*} M\right)=\mathscr{R}\left(A^{*}\right) \cap \mathscr{R}(\widehat{B})$ and $\mathscr{R}\left(\widehat{B} M^{*}\right)=\mathscr{R}(\widehat{B}) \cap \mathscr{R}\left(A^{*}\right)$.

$\langle 67\rangle$ Both $r\left[A^{*} M, \widehat{B}\right]=r(\widehat{B})$ and $r\left[\widehat{B} M^{*}, A^{*}\right]=r(A)$.

$\langle 68\rangle r\left[A^{*}, \widehat{B}\right]=r(A)+r(\widehat{B})-r(M), r\left[A A^{*} M, M\right]=r(M)$, and $r\left[\widehat{B}^{*} \widehat{B} M^{*}, M^{*}\right]=r(M)$.

$\langle 69\rangle r\left[\begin{array}{cc}M M^{*} M & M \widehat{B}^{*} \widehat{B} \\ A A^{*} M & M\end{array}\right]=r(M)$

$\langle 70\rangle M \widehat{B}^{*} \widehat{B} M^{\dagger} A A^{*} M=M M^{*} M, r\left[A A^{*} M, M\right]=r(M)$, and $r\left[\widehat{B}^{*} \widehat{B} M^{*}, M^{*}\right]=r(M)$.

$\langle 71\rangle r\left[\begin{array}{cc}M M^{*} M & M \widehat{B}^{*} \widehat{B} \\ A A^{*} M & M\end{array}\right]=r\left[A^{*}, \widehat{B}\right]+2 r(M)-r(A)-r(\widehat{B})$ and $r\left[A^{*} M, \widehat{B}\right]+r\left[\widehat{B} M^{*}, A^{*}\right]=r\left[A^{*}, \widehat{B}\right]+r(M)$. 
$\langle 72\rangle r\left[\begin{array}{cc}M M^{*} M & M \widehat{B}^{*} \widehat{B} \\ A A^{*} M & M\end{array}\right]=r\left[A^{*} M, \widehat{B}\right]+r\left[\widehat{B} M^{*}, A^{*}\right]+r(A)+r(\widehat{B})-2 r\left[A^{*}, \widehat{B}\right]-r(A \widehat{B}), r\left[A A^{*} M, M\right]=r(M)$, and $r\left[\widehat{B}^{*} \widehat{B} M^{*}, M^{*}\right]=r(M)$.

$\langle 73\rangle$ The matrix equation $\widehat{B} X A=A^{*} A \widehat{B} \widehat{B}^{*}$ is consistent.

Corollary 11.6. Let $A \in \mathbb{C}^{m \times n}$ and $B \in \mathbb{C}^{n \times p}$, and denote $\widetilde{A}=\left(A^{*} A\right)^{\frac{1}{2}}, \widetilde{B}=\left(B B^{*}\right)^{\frac{1}{2}}$, and $M=\widetilde{A} \widetilde{B}$. Then the following 74 statements are equivalent:

$\langle 0\rangle(A B)^{\dagger}=B^{\dagger} A^{\dagger}$.

$\langle 2\rangle M^{\dagger} \in\left\{\widetilde{B}^{\dagger} \widetilde{A}^{(1,3,4)}\right\}$.

$\langle 4\rangle M^{\dagger} \in\left\{\widetilde{B}^{\dagger} \widetilde{A}^{(1,4)}\right\}$.

$\langle 6\rangle M^{\dagger} \in\left\{\widetilde{B}^{(1,3,4)} \widetilde{A}^{(1,3,4)}\right\}$.

$\langle 8\rangle M^{\dagger} \in\left\{\widetilde{B}^{(1,3,4)} \widetilde{A}^{(1,4)}\right\}$.

$\langle 10\rangle M^{\dagger} \in\left\{\widetilde{B}^{(1,2,3)} \widetilde{A}^{(1,3,4)}\right\}$.

$\langle 12\rangle M^{\dagger} \in\left\{\widetilde{B}^{(1,2,3)} \widetilde{A}^{(1,4)}\right\}$.

$\langle 14\rangle M^{\dagger} \in\left\{\widetilde{B}^{(1,3)} \widetilde{A}^{(1,3,4)}\right\}$.

$\langle 16\rangle M^{\dagger} \in\left\{\widetilde{B}^{(1,3)} \widetilde{A}^{(1,4)}\right\}$.

$\langle 18\rangle\left\{M^{(1,3,4)}\right\} \supseteq\left\{\widetilde{B}^{(1,3,4)} \widetilde{A}^{\dagger}\right\}$.

$\langle 20\rangle$ Both $\left\{M^{(1,3)}\right\} \ni \widetilde{B}^{\dagger} \widetilde{A}^{\dagger}$ and $\left\{M^{(1,4)}\right\} \ni \widetilde{B}^{\dagger} \widetilde{A}^{\dagger}$.

$\langle 21\rangle$ Both $\left\{M^{(1,3)}\right\} \supseteq\left\{\widetilde{B}^{(1,3,4)} \widetilde{A}^{\dagger}\right\}$ and $\left\{M^{(1,4)}\right\} \supseteq\left\{\widetilde{B}^{\dagger} \widetilde{A}^{(1,3,4)}\right\}$.

$\langle 22\rangle$ Both $\left\{M^{(1,3)}\right\} \supseteq\left\{\widetilde{B}^{(1,2,3)} \widetilde{A}^{\dagger}\right\}$ and $\left\{M^{(1,4)}\right\} \supseteq\left\{\widetilde{B}^{\dagger} \widetilde{A}^{(1,2,4)}\right\}$.

$\langle 23\rangle$ Both $\left\{M^{(1,3)}\right\} \supseteq\left\{\widetilde{B}^{(1,3)} \widetilde{A}^{\dagger}\right\}$ and $\left\{M^{(1,4)}\right\} \supseteq\left\{\widetilde{B}^{\dagger} \widetilde{A}^{(1,4)}\right\}$.

$\langle 24\rangle$ Both $\left\{M^{(1,3)}\right\} \supseteq\left\{\widetilde{B}^{\dagger} \widetilde{A}^{(1,3,4)}\right\}$ and $\left\{M^{(1,4)}\right\} \supseteq\left\{\widetilde{B}^{(1,3,4)} \widetilde{A}^{\dagger}\right\}$.

$\langle 25\rangle$ Both $\left\{M^{(1,3)}\right\} \supseteq\left\{\widetilde{B}^{(1,3,4)} \widetilde{A}^{(1,3,4)}\right\}$ and $\left\{M^{(1,4)}\right\} \supseteq\left\{\widetilde{B}^{(1,3,4)} \widetilde{A}^{(1,3,4)}\right\}$.

$\langle 26\rangle$ Both $\left\{M^{(1,3)}\right\} \supseteq\left\{\widetilde{B}^{(1,2,3)} \widetilde{A}^{(1,3,4)}\right\}$ and $\left\{M^{(1,4)}\right\} \supseteq\left\{\widetilde{B}^{(1,3,4)} \widetilde{A}^{(1,2,4)}\right\}$.

$\langle 27\rangle$ Both $\left\{M^{(1,3)}\right\} \supseteq\left\{\widetilde{B}^{(1,3)} \widetilde{A}^{(1,3,4)}\right\}$ and $\left\{M^{(1,4)}\right\} \supseteq\left\{\widetilde{B}^{(1,3,4)} \widetilde{A}^{(1,3)}\right\}$.

$\langle 28\rangle$ Both $\left\{M^{(1,3)}\right\} \supseteq\left\{\widetilde{B}^{\dagger} \widetilde{A}^{(1,2,3)}\right\}$ and $\left\{M^{(1,4)}\right\} \supseteq\left\{\widetilde{B}^{(1,2,4)} \widetilde{A}^{\dagger}\right\}$.

$\langle 29\rangle$ Both $\left\{M^{(1,3)}\right\} \supseteq\left\{\widetilde{B}^{(1,3,4)} \widetilde{A}^{(1,2,3)}\right\}$ and $\left\{M^{(1,4)}\right\} \supseteq\left\{\widetilde{B}^{(1,2,4)} \widetilde{A}^{(1,3,34)}\right\}$.

$\langle 30\rangle$ Both $\left\{M^{(1,3)}\right\} \supseteq\left\{\widetilde{B}^{(1,2,3)} \widetilde{A}^{(1,2,3)}\right\}$ and $\left\{M^{(1,4)}\right\} \supseteq\left\{\widetilde{B}^{(1,2,4)} \widetilde{A}^{(1,2,4)}\right\}$.

$\langle 31\rangle$ Both $\left\{M^{(1,3)}\right\} \supseteq\left\{\widetilde{B}^{(1,3)} \widetilde{A}^{(1,2,3)}\right\}$ and $\left\{M^{(1,4)}\right\} \supseteq\left\{\widetilde{B}^{(1,2,4)} \widetilde{A}^{(1,4)}\right\}$.

$\langle 32\rangle$ Both $\left\{M^{(1,3)}\right\} \supseteq\left\{\widetilde{B}^{\dagger} \widetilde{A}^{(1,3)}\right\}$ and $\left\{M^{(1,4)}\right\} \supseteq\left\{\widetilde{B}^{(1,4)} \widetilde{A}^{\dagger}\right\}$.

$\langle 33\rangle$ Both $\left\{M^{(1,3)}\right\} \supseteq\left\{\widetilde{B}^{(1,3,4)} \widetilde{A}^{(1,3)}\right\}$ and $\left\{M^{(1,4)}\right\} \supseteq\left\{\widetilde{B}^{(1,4)} \widetilde{A}^{(1,3,4)}\right\}$.

$\langle 34\rangle$ Both $\left\{M^{(1,3)}\right\} \supseteq\left\{\widetilde{B}^{(1,2,3)} \widetilde{A}^{(1,3)}\right\}$ and $\left\{M^{(1,4)}\right\} \supseteq\left\{\widetilde{B}^{(1,4)} \widetilde{A}^{(1,2,4)}\right\}$. 
$\langle 35\rangle$ Both $\left\{M^{(1,3)}\right\} \supseteq\left\{\widetilde{B}^{(1,3)} \widetilde{A}^{(1,3)}\right\}$ and $\left\{M^{(1,4)}\right\} \supseteq\left\{\widetilde{B}^{(1,4)} \widetilde{A}^{(1,4)}\right\}$.

$\langle 36\rangle\left\{(\widetilde{A} M)^{(1)}\right\} \ni \widetilde{B}^{\dagger}\left(\widetilde{A}^{2}\right)^{\dagger},\left\{(\widetilde{A} M)^{(1,3)}\right\} \ni M^{\dagger}(\widetilde{A})^{\dagger},\left\{(M \widetilde{B})^{(1)}\right\} \ni\left(\widetilde{B}^{2}\right)^{\dagger} \widetilde{A}^{\dagger}$, and $\left\{(M \widetilde{B})^{(1,4)}\right\} \ni \widetilde{B}^{\dagger} M^{\dagger}$.

$\langle 37\rangle M^{\dagger}=\widetilde{B}^{\dagger} \widetilde{A}^{\dagger} M \widetilde{B}^{\dagger} \widetilde{A}^{\dagger} . \quad\langle 38\rangle \widetilde{B} M^{\dagger} \widetilde{A}=\widetilde{B} \widetilde{B}^{\dagger} \widetilde{A}^{\dagger} \widetilde{A} . \quad\langle 39\rangle \widetilde{B}^{2} M^{\dagger} \widetilde{A}^{2}=M^{*}$.

$\langle 40\rangle$ Both $M M^{\dagger}=M \widetilde{B}^{\dagger} \widetilde{A}^{\dagger}$ and $M^{\dagger} M=\widetilde{B}^{\dagger} \widetilde{A}^{\dagger} M$.

$\langle 41\rangle$ Both $M M^{\dagger} \widetilde{A}=M \widetilde{B}^{\dagger}$ and $\widetilde{A}^{\dagger} M=\widetilde{B} M^{\dagger} M$.

$\langle 42\rangle$ Both $\widetilde{B} \widetilde{B}^{\dagger} \widetilde{A}^{2} \widetilde{B}=\widetilde{A}^{2} \widetilde{B}$ and $\widetilde{A} \widetilde{B}^{2} \widetilde{A}^{\dagger} \widetilde{A}=\widetilde{A} \widetilde{B}^{2}$.

$\langle 43\rangle \widetilde{B} \widetilde{B}^{\dagger} \widetilde{A}^{2} \widetilde{B}^{2} \widetilde{A}^{\dagger} \widetilde{A}=\widetilde{B} \widetilde{B}^{\dagger} \widetilde{A}^{2} \widetilde{B}^{2}=\widetilde{A}^{2} \widetilde{B}^{2} \widetilde{A}^{\dagger} \widetilde{A}=\widetilde{A}^{2} \widetilde{B}^{2}$.

$\langle 44\rangle$ Both $M^{\dagger}=\left(\widetilde{A}^{\dagger} \widetilde{A} \widetilde{B}\right)^{\dagger} \widetilde{A}^{\dagger}$ and $\left(\widetilde{A^{\dagger}} \widetilde{A} \widetilde{B}\right)^{\dagger}=\widetilde{B}^{\dagger} \widetilde{A}^{\dagger} \widetilde{A}$.

$\langle 45\rangle$ Both $M^{\dagger}=\widetilde{B}^{\dagger}\left(\widetilde{A} \widetilde{B} \widetilde{B}^{\dagger}\right)^{\dagger}$ and $\left(\widetilde{A} \widetilde{B} \widetilde{B}^{\dagger}\right)^{\dagger}=\widetilde{B} \widetilde{B}^{\dagger} \widetilde{A}^{\dagger}$.

$\langle 46\rangle$ Both $M^{\dagger}=\left(\widetilde{A}^{2} \widetilde{B}\right)^{\dagger} \widetilde{A}$ and $\left(\widetilde{A}^{2} \widetilde{B}\right)^{\dagger}=\widetilde{B}^{\dagger}\left(\widetilde{A}^{2}\right)^{\dagger}$.

$\langle 47\rangle$ Both $M^{\dagger}=\widetilde{B}\left(\widetilde{A} \widetilde{B}^{2}\right)^{\dagger}$ and $\left(\widetilde{A} \widetilde{B}^{2}\right)^{\dagger}=\left(\widetilde{B}^{2}\right)^{\dagger} \widetilde{A}^{\dagger}$.

$\langle 48\rangle$ Both $M^{\dagger}=\widetilde{B}^{\dagger}\left(\widetilde{A^{\dagger}} \widetilde{A} \widetilde{B} \widetilde{B}^{\dagger}\right)^{\dagger} \widetilde{A}^{\dagger}$ and $\left(\widetilde{A^{\dagger}} \widetilde{A} \widetilde{B} \widetilde{B}^{\dagger}\right)^{\dagger}=\widetilde{B} \widetilde{B}^{\dagger} \widetilde{A}^{\dagger} \widetilde{A}$.

$\langle 49\rangle$ Both $M^{\dagger}=\widetilde{B}^{2 k-1}\left(\widetilde{A}^{2 k} \widetilde{B}^{2 k}\right)^{\dagger} \widetilde{A}^{2 k-1}$ and $\left(\widetilde{A}^{2 k} \widetilde{B}^{2 k}\right)^{\dagger}=\left(\widetilde{B}^{2 k}\right)^{\dagger}\left(\widetilde{A}^{2 k}\right)^{\dagger}$ for any integer $k \geq 1$.

$\langle 50\rangle$ Both $M^{\dagger}=\widetilde{B}^{2}\left(\widetilde{A}^{3} \widetilde{B}^{3}\right)^{\dagger} \widetilde{A}^{2}$ and $\left(\widetilde{A}^{3} \widetilde{B}^{3}\right)^{\dagger}=\left(\widetilde{B}^{3}\right)^{\dagger}\left(\widetilde{A}^{3}\right)^{\dagger}$.

$\langle 51\rangle \operatorname{Both}\left(\widetilde{A} \widetilde{B} \widetilde{B}^{\dagger}\right)^{\dagger}=\widetilde{B} \widetilde{B}^{\dagger} \widetilde{A}^{\dagger}$ and $\left(\widetilde{A^{\dagger}} \widetilde{A} \widetilde{B}\right)^{\dagger}=\widetilde{B}^{\dagger} \widetilde{A}^{\dagger} \widetilde{A}$.

$\langle 52\rangle$ Both $M \widetilde{B}^{\dagger} \widetilde{A}^{\dagger}$ and $\widetilde{B}^{\dagger} \widetilde{A}^{\dagger} M$ are orthogonal projectors.

$\langle 53\rangle$ Both $\widetilde{A} E_{\widetilde{B}} \widetilde{A}^{\dagger}$ and $\widetilde{B}^{\dagger} F_{\widetilde{A}} \widetilde{B}$ are orthogonal projectors.

$\langle 54\rangle$ Both $M M^{\dagger} \widetilde{A}=M \widetilde{B}^{\dagger}$ and $\widetilde{B} M^{\dagger} M=\widetilde{A}^{\dagger} M$.

$\langle 55\rangle$ Both $\widetilde{A}^{2} \widetilde{B} \widetilde{B}^{\dagger}=\widetilde{B} \widetilde{B}^{\dagger} \widetilde{A}^{2}$ and $\widetilde{A^{\dagger}} \widetilde{A} \widetilde{B}^{2}=\widetilde{B}^{2} \widetilde{A}^{\dagger} \widetilde{A}$.

$\langle 56\rangle$ Both $M \widetilde{B}^{\dagger} \widetilde{A}^{\dagger} M=M$ and $M^{\dagger}=\left(\widetilde{A}^{\dagger} M\right)^{\dagger} \widetilde{A}^{\dagger}=\widetilde{B}^{\dagger}\left(M \widetilde{B}^{\dagger}\right)^{\dagger}$.

$\langle 57\rangle$ Both $M \widetilde{B}^{\dagger} \widetilde{A}^{\dagger} M=M$ and $M^{\dagger}=(\widetilde{A} M)^{\dagger} \widetilde{A}=\widetilde{B}(M \widetilde{B})^{\dagger}$.

$\langle 58\rangle M \widetilde{B}^{\dagger} \widetilde{A}^{\dagger} M=M,\left(\widetilde{A}^{\dagger} M\right)^{\dagger}=M^{\dagger} \widetilde{A}$, and $\left(M \widetilde{B}^{\dagger}\right)^{\dagger}=\widetilde{B} M^{\dagger}$.

$\langle 59\rangle M \widetilde{B}^{\dagger} \widetilde{A}^{\dagger} M=M,\left(\widetilde{A}^{\dagger} M M^{\dagger} \widetilde{A}\right)^{*}=\widetilde{A}^{\dagger} M M^{\dagger} \widetilde{A}$, and $\left(\widetilde{B} M^{\dagger} M \widetilde{B}^{\dagger}\right)^{*}=\widetilde{B} M^{\dagger} M \widetilde{B}^{\dagger}$.

$\langle 60\rangle M \widetilde{B}^{\dagger} \widetilde{A}^{\dagger} M=M, M M^{\dagger} \widetilde{A} \widetilde{A}=\widetilde{A} \widetilde{A} M M^{\dagger}$, and $M^{\dagger} M \widetilde{B} \widetilde{B}=\widetilde{B} \widetilde{B} M^{\dagger} M$.

$\langle 61\rangle$ Both $\mathscr{R}\left(\widetilde{A}^{2} \widetilde{B} \widetilde{B}^{\dagger}\right)=\mathscr{R}\left(\widetilde{B} \widetilde{B}^{\dagger} \widetilde{A}^{2}\right)$ and $\mathscr{R}\left(\widetilde{A} \dagger \widetilde{A} \widetilde{B}^{2}\right)=\mathscr{R}\left(\widetilde{B}^{2} \widetilde{A}^{\dagger} \widetilde{A}\right)$.

$\langle 62\rangle \mathscr{R}\left(\widetilde{A^{\dagger}} \widetilde{A} \widetilde{B} \widetilde{B}^{\dagger}\right)=\mathscr{R}\left(\widetilde{B} \widetilde{B}^{\dagger} \widetilde{A}^{\dagger} \widetilde{A}\right), \mathscr{R}\left(M^{\dagger}\right)=\mathscr{R}\left(\widetilde{B}^{\dagger} \widetilde{A}^{\dagger}\right)$, and $\mathscr{R}\left[\left(M^{*}\right)^{\dagger}\right]=\mathscr{R}\left[(\widetilde{A})^{\dagger}(\widetilde{B})^{\dagger}\right]$.

$\langle 63\rangle$ Both $\mathscr{R}\left(\widetilde{A} \dagger \widetilde{A} \widetilde{B} \widetilde{B}^{\dagger}\right)=\mathscr{R}\left(\widetilde{B}^{2} \widetilde{A}^{2}\right)$ and $\mathscr{R}\left(\widetilde{B} \widetilde{B}^{\dagger} \widetilde{A}^{\dagger} \widetilde{A}\right)=\mathscr{R}\left(\widetilde{A}^{2} \widetilde{B}^{2}\right)$.

$\langle 64\rangle \mathscr{R}\left(\widetilde{A}^{2} \widetilde{B}^{2}\right)=\mathscr{R}\left(\widetilde{B}^{2} \widetilde{A}^{2}\right)$.

$\langle 65\rangle$ Both $\mathscr{R}(\widetilde{A} M) \subseteq \mathscr{R}(\widetilde{B})$ and $\mathscr{R}\left(\widetilde{B} M^{*}\right) \subseteq \mathscr{R}(\widetilde{A})$.

$\langle 66\rangle$ Both $\mathscr{R}(\widetilde{A} M)=\mathscr{R}(\widetilde{A}) \cap \mathscr{R}(\widetilde{B})$ and $\mathscr{R}\left(\widetilde{B} M^{*}\right)=\mathscr{R}(\widetilde{B}) \cap \mathscr{R}(\widetilde{A})$.

$\langle 67\rangle$ Both $r[\widetilde{A} M, \widetilde{B}]=r(\widetilde{B})$ and $r\left[\widetilde{B} M^{*}, \widetilde{A}\right]=r(\widetilde{A})$.

$\langle 68\rangle r[\widetilde{A}, \widetilde{B}]=r(\widetilde{A})+r(\widetilde{B})-r(M), r\left[\widetilde{A}^{2} M, M\right]=r(M)$, and $r\left[\widetilde{B}^{2} M^{*}, M^{*}\right]=r(M)$.

$\langle 69\rangle r\left[\begin{array}{cc}M M^{*} M & M \widetilde{B}^{2} \\ \widetilde{A}^{2} M & M\end{array}\right]=r(M)$. 
$\langle 70\rangle M \widetilde{B}^{2} M^{\dagger} \widetilde{A}^{2} M=M M^{*} M, r\left[\widetilde{A}^{2} M, M\right]=r(M)$, and $r\left[\widetilde{B}^{2} M^{*}, M^{*}\right]=r(M)$.

$\langle 71\rangle r\left[\begin{array}{cc}M M^{*} M & M \widetilde{B}^{2} \\ \widetilde{A}^{2} M & M\end{array}\right]=r[\widetilde{A}, \widetilde{B}]+2 r(M)-r(\widetilde{A})-r(\widetilde{B})$ and $r[\widetilde{A} M, \widetilde{B}]+r\left[\widetilde{B} M^{*}, \widetilde{A}\right]=r[\widetilde{A}, \widetilde{B}]+r(M)$.

$\langle 72\rangle r\left[\begin{array}{cc}M M^{*} M & M \widetilde{B}^{2} \\ \widetilde{A}^{2} M & M\end{array}\right]=r[\widetilde{A} M, \widetilde{B}]+r\left[\widetilde{B} M^{*}, \widetilde{A}\right]+r(\widetilde{A})+r(\widetilde{B})-2 r[\widetilde{A}, \widetilde{B}]-r(\widetilde{A} \widetilde{B}), r\left[\widetilde{A}^{2} M, M\right]=r(M)$, and $r\left[\widetilde{B}^{2} M^{*}, M^{*}\right]=r(M)$.

$\langle 73\rangle$ The matrix equation $\widetilde{B} X \widetilde{A}=\widetilde{A}^{2} \widetilde{B}^{2}$ is consistent.

\section{Concluding remarks}

We have formulated several groups of two-term ROLs for generalized inverses of products of two matrices, given a complete account of the one-sided matrix set inclusions in (1.9) by means of the three classic block matrix method, matrix rank method, and matrix equation method in matrix calculus, which have been identified as dependable and efficient tools for dealing ROLs in most situations. We believe that the whole work will have certain influential impact on the development of the theory of matrix equalities/identities and the theory of matrix ranks.

It is undoubtedly a fundament work to establish equalities/identities for elements in algebras of other types, and it is easy to see that all the preceding results and facts can symbolically be extended to the analogous topics on ROLs in other algebraic structures that are somehow close to the matrix case, in which generalized inverses of elements are defined by the four Penrose equations as well. But it should be pointed out that for the same kind of ROL problems, any results derived from methods other than the three BMM, MRM, and MEM for a given ROL to hold over general algebraic structures that are close to the matrix case must be consistent with these deduced from the three careful matrix analytic methods for real or complex matrices. The past and present works together with the countless open problems proposed demonstrate once again that algebraic equality/identity problems are a class of featured subjects in mathematics and will attract ever-lasting and prominent attention in different research fields of mathematics.

\section{References}

[1] E. Arghiriade. Remarques sur l'inverse généralisée d'un produit de matrices. Atti Accad. Naz. Lincei Rend. Cl. Sci. Fis. Mat. Natur. Ser. VIII 42(1967), 621-625.

[2] A. Arias, S. Gudder. Almost sharp quantum effects. J. Math. Phys. 45(2004), 4196-4206.

[3] J.K. Baksalary. Algebraic characterizations and statistical implications of the commutativity of orthogonal projectors. In Proceedings of the Second International Tampere Conference in Statistics (T. Pukkila and S. Puntanen eds.), University of Tampere, Tampere, Finland, 1987, pp. 113-142.

[4] J.K. Baksalary, O.M. Baksalary. Commutativity of projectors. Linear Algebra Appl. 341(2002), $129-142$.

[5] J.K. Baksalary, O.M. Baksalary, T. Szulc. A property of orthogonal projectors. Linear Algebra Appl. 354(2002), 35-39.

[6] J.K. Baksalary, G.P.H. Styan. Around a formula for the rank of a matrix product with some statistical applications. In Graphs, Matrices, and Designs: Festschrift in Honor of N.J. Pullman on his Sixtieth Birthday (R.S. Rees ed.), Marcel Dekker, New York, 1993, pp. 1-18.

[7] J.K. Baksalary, O.M. Baksalary. An invariance property related to the reverse order law. Linear Algebra Appl. 410(2005), 64-69.

[8] J.K. Baksalary, G.P.H. Styan. Around a formula for the rank of a matrix product with some statistical applications. In: R.S. Rees (Ed.), Graphs, Matrices, and Designs: Festschrift in Honor of N.J. Pullman on his Sixtieth Birthday, Marcel Dekker, New York, 1993, pp. 1-18.

[9] O.M. Baksalary, G. Trenkler. An alternative approach to characterize the commutativity of orthogonal projectors. Discuss. Math. Prob. Stat. 28(2008), 113-137.

[10] O.M. Baksalary, G. Trenkler. Column space equalities for orthogonal projectors. Appl. Math. Comput. 212(2009), $519-529$.

[11] O.M. Baksalary, G. Trenkler. On angles and distances between subspaces. Linear Algebra Appl. 431(2009), 2243-2260.

[12] O.M. Baksalary, G. Trenkler. On a subspace metric based on matrix rank. Linear Algebra Appl. 432(2010), $1475-1491$.

[13] O.M. Baksalary, G. Trenkler. On the projectors $F F^{\dagger}$ and $F^{\dagger} F$. Appl. Math. Comput. 217(2011), 10213-10223.

[14] D.T. Barwick, J.D. Gilbert. Generalization of the reverse order law with related results. Linear Algebra Appl. 8(1974), $345-349$.

[15] D.T. Barwick, J.D. Gilbert. On generalizations of the reverse order law with related results. SIAM. J. Appl. Math. $27(1974), 326-330$.

[16] A. Ben-Israel, T.N.E. Greville. Generalized Inverses: Theory and Applications. 2nd ed., Springer, New York, 2003. 
[17] A.M. Bikchentaev. Commutativity of projectors and trace characterization on von Neumann algebras I. Russian Math. $53(2009), 68-71$.

[18] N.H. Bingham, J.M. Fry. Regression: Linear Models in Statistics. Springer, London, 2010.

[19] S.L. Campbell, C.D. Meyer. Generalized Inverses of Linear Transformations. SIAM, Philadephia, 2009.

[20] S. Cheng, Y. Tian. Moore-Penrose inverses of products and differences of orthogonal projectors. Acta Sci. Math. (Szeged) 69(2003), 533-542.

[21] D.S. Cvetković-Ilić. Reverse order laws for $\{1,3,4\}$-generalized inverses in $C^{*}$-algebras. Applied Math. Lett. 24(2011), 210-213.

[22] D.S. Cvetković-Ilić, M. Djikić. Various solutions to reverse order law problems. Linear Multilinear Algebra 64(2016), 1207-1219.

[23] D.S. Cvetković-Ilić, J. Milošević. Reverse order laws for $\{1,3\}$-generalized inverses. Linear Multilinear Algebra 67(2019), 613-624.

[24] D.S. Cvetković-Ilić, Y. Wei. Algebraic Properties of Generalized Inverses. Springer, Singapore, 2017.

[25] Jan de Leeuw, E. Meijer. Handbook of Multilevel Analysis. Springer, New York, 2008.

[26] E. Demidenko. Mixed Models: Theory and Applications. Wiley, New York, 2004.

[27] N.Č. Dinčić, D.S. Djordjević. Basic reverse order law and its equivalencies[J]. Aequat. math. 85(2013), 505-517.

[28] N.Č. Dinčić, D.S. Djordjević, D. Mosić. Mixed-type reverse order law and its equivalents. Studia Math. 204(2011), $123-136$.

[29] I. Erdelyi. On the "reverse order law" related to the generalized inverse of matrix products. J. ACM 13(1966), 439-443.

[30] M. Fazel, T.K. Pong, D. Sun, P. Tseng. Hankel matrix rank minimization with applications to system identification and realization. SIAM J. Matrix Anal. Appl. 34(2013), 946-977.

[31] A.M. Galperin, Z. Waksman. On pseudo inverse of operator products. Linear Algebra Appl. 33(1980), $123-131$.

[32] T.N.E. Greville. Note on the generalized inverse of a matrix product. SIAM Rev. 8(1966), 518-521.

[33] H. Goldstein. Multilevel Statistical Models. 4th ed., Wiley, 2011.

[34] F.A. Graybill. An Introduction to Linear Statistical Models. Vol. I, McGraw-Hill, 1961.

[35] J. Groß. Some remarks concerning the reverse order law. Discuss. Math. Algebra Stochastic Methods. 17(1997), 135-141.

[36] J. Groß. On the product of orthogonal projectors. Linear Algebra Appl. 289(1999), 141-150.

[37] J. Groß, Y. Tian. Invariance properties of a triple matrix product involving generalized inverses. Linear Algebra Appl. 417(2006), 94-107.

[38] R.E. Hartwig. The reverse order law revisited. Linear Algebra Appl. 76(1986), 241-246.

[39] R.E. Hartwig. K. Spindelböck. Matrices for which $A^{*}$ and $A^{\dagger}$ can commute. Linear Multilinear Algebra 14(1984), $241-256$.

[40] S. Izumino. The product of operators with closed range and an extension of the reverse order law. Tôhoku Math. J. 34(1982), 43-52.

[41] B. Jiang, Y. Tian. Necessary and sufficient conditions for nonlinear matrix identities to always hold. Aequat. Math. 93(2019), 587-600.

[42] K. Konishi, K. Uruma, T. Takahashi, T. Furukawa. Iterative partial matrix shrinkage algorithm for matrix rank minimization. Sign. Proces. 100(2014), 124-131.

[43] G.G. Kreft, J. de Leeuw. Introduction to Multilevel Modelling. Sage Publications, Thousand Oaks, CA, 1998.

[44] S.D. Liu, H. You. Reverse order law for generalized inverses and reflexive generalized inverses and reflexive generalized inverses of products of matrices over arbitrary fields (in Chinese). Chin. Ann. of Math. Ser. A 25(2004), 523-530.

[45] Y. Liu, Y. Tian. A mixed-type reverse order law for generalized inverses of triple matrix products (in Chinese). Acta Math. Sinica 52(2009), 197-204.

[46] X. Liu, S. Huang, D.S. Cvetković-Ilić. Mixed-type reverse-order laws for $\{1,3,4\}$-generalized inverses over Hilbert spaces. Applied Math. Comput. 218(2012), 8570-8577.

[47] X. Liu, H. Yang. A note on the reverse order laws for $\{1,2,3\}$ - and $\{1,2,4\}$-inverses of multiple matrix products. Electron. J. linear Algebra 22(2011), 620-629.

[48] X. Liu, S. Wu, D.S. Cvetković-Ilić. New results on reverse order law for $\{1,2,3\}$ - and $\{1,2,4\}$-inverses of bounded operators. Math. Comput. 82(2013), 1597-1607.

[49] N.T. Longford. Random Coefficient Models. Oxford University Press, Oxford, 1993.

[50] S.Q. Ma, D. Goldfarb, L.F. Chen, Fix point and Bregman iterative methods for matrix rank minimization. Math. Prog. 128(2011), 321-353.

[51] G. Marsaglia, G.P.H. Styan. Equalities and inequalities for ranks of matrices. Linear Multilinear Algebra 2(1974), 269-292.

[52] K. Mohan, M. Fazel. Iterative reweighted algorithms for matrix rank minimization. J. Mach Learn. Res. 13(2012), $3441-3473$.

[53] K. Nordström, D. von Rosen. Algebra of subspaces with applications to problems in statistics. In: Proceedings of the Second International Tampere Conference in Statistics (T. Pukkila and S. Puntanen eds.), University of Tampere, Tampere, Finland, 1987, pp. 603-614.

[54] R. Penrose. A generalized inverse for matrices. Proc. Cambridge Phil. Soc. 51(1955), 406-413. 
[55] S. Puntanen, G.P.H. Styan, J. Isotalo. Matrix Tricks for Linear Statistical Models: Our Personal Top Twenty. Springer, Berlin, 2011.

[56] J.N. Radenković. Reverse order law for generalized inverses of multiple operator product. Linear Multilinear Algebra 64(2016), 1266-1282.

[57] C.R. Rao, S.K. Mitra. Generalized Inverse of Matrices and Its Applications. Wiley, New York, 1971.

[58] C.R. Rao, S.K. Mitra, P. Bhimasankaram. Determination of a matrix by its subclasses of generalized inverses, Sankhyā Ser. A 34(1972), 5-8.

[59] S.W. Raudenbush, A.S. Bryk. Hierarchical Linear Models: Applications and Data Analysis Methods. 2nd ed, Sage, London, 2002.

[60] B. Recht, M. Fazel, P.A. Parrilo. Guaranteed minimum-rank solutions of linear matrix equations via nuclear norm minimization. SIAM Review 52(2010), 471-501.

[61] W. Rehder. On the commutativity of two projections. Elem. Math. 35(1980), 120-122.

[62] W. Rehder. When do projections commute? Z. Naturforsch. A. 35(1980), 437-441.

[63] S.R. Searle. Linear Models. Wiley, New York, 1971.

[64] T.A.B. Snijders, R.J. Bosker. Multilevel Analysis: An Introduction to Basic and Advanced Multilevel Modeling. 2nd ed., Sage Publications, London, 1999.

[65] N. Shinozaki, M. Sibuya. The reverse order law $(A B)^{-}=B^{-} A^{-}$. Linear Algebra Appl. 9(1974), $29-40$.

[66] N. Shinozaki, M. Sibuya. Further results on the reverse order law. Linear Algebra Appl. 27(1979), 9-16.

[67] M. Sibuya. Subclasses of generalized inverses of matrices. Ann. Instit. Statist. Math. 22(1970), 543-556.

[68] M.I. Smith. A Schur algorithm for computing matrix pth roots. SIAM J. Matrix Anal. Appl. 24(2003), 971-989.

[69] E. Størmer. Positive maps which map the set of rank k projections onto itself. Positivity 21(2016), 509511.

[70] G.P.H. Styan. Schur complements and linear models. In: Proc. First International Tampere Seminar on Linear Statistical Models and Their Applications, Dept. of Mathematical Science, University of Tampere, Finland, 1985 , pp. 37-75.

[71] Y. Takane, Y. Tian, H. Yanai. On reverse-order laws for least-squares $g$-inverses and minimum norm $g$-inverses of a matrix product. Aequat. Math.73(2007), 56-70.

[72] Y. Tian. The Moore-Penrose inverse of a triple matrix product (in Chinese). Math. Theory Practice 1(1992), 64-70.

[73] Y. Tian. Reverse order laws for the generalized inverses of multiple matrix products. Linear Algebra Appl. 211(1994), $85-100$.

[74] Y. Tian. Rank equalities related to outer inverses of matrices and applications. Linear Multilinear Algebra 49(2002), $269-288$.

[75] Y. Tian. The maximal and minimal ranks of some expressions of generalized inverses of matrices. Southeast Asian Bull. Math. 25(2002), 745-755.

[76] Y. Tian. Reverse order laws for the weighted Moore-Penrose inverse of a triple matrix product with applications. Internat. Math. J. 3(2003), 107-117.

[77] Y. Tian. Reverse order laws for the Drazin inverse of a triple matrix product. Pub. Math. Debrecen 63(2003), 261-277.

[78] Y. Tian. Using rank formulas to characterize equalities for Moore-Penrose inverses of matrix products. Appl. Math. Comput. 147(2004), 581-600.

[79] Y. Tian. More on maximal and minimal ranks of Schur complements with applications. Appl. Math. Comput. 152(2004), 675-692.

[80] Y. Tian. On mixed-type reverse-order laws for the Moore-Penrose inverse of a matrix product. Internat. J. Math. Math. Sci. 58(2004), 3103-3116.

[81] Y. Tian. The reverse-order law $(A B)^{\dagger}=B^{\dagger}\left(A^{\dagger} A B B^{\dagger}\right)^{\dagger} A^{\dagger}$ and its equivalent equalities. J. Math. Kyoto Univ. 45(2005), $841-850$.

[82] Y. Tian. The Moore-Penrose inverse for sums of matrices under rank additivity conditions. Linear Multilinear Algebra $53(2005), 45-65$.

[83] Y. Tian. The equivalence between $(A B)^{\dagger}=B^{\dagger} A^{\dagger}$ and other mixed-type reverse-order laws. Int. J. Math. Educ. Sci. Technol. 37(2006), 331-339.

[84] Y. Tian. Some mixed-type reverse-order laws for the Moore-Penrose inverse of a triple matrix product. Rocky Mt. J. Math. 37(2007), 1327-1347.

[85] Y. Tian. Extremal ranks of a quadratic matrix expression with applications. Linear Multilinear Algebra 59(2011), $627-644$.

[86] Y. Tian. Equalities and inequalities for ranks of products of generalized inverses of two matrices and their applications. J. Inequal. Appl. 182(2016), 1-51.

[87] Y. Tian. How to establish exact formulas for calculating the max-min ranks of products of two matrices and their generalized inverses. Linear Multilinear Algebra 66(2018), 22-73.

[88] Y. Tian. Formulas for calculating the dimensions of the sums and the intersections of a family of linear subspaces with applications. Beitr. Algebra Geom. 60(2019), 471-485.

[89] Y. Tian. On relationships between two linear subspaces and two orthogonal projectors. Spec. Matrices 7(2019), 142-212.

[90] Y. Tian. Classification analysis to the equalities $A^{(i, \ldots, j)}=B^{(k, \ldots, l)}$ for generalized inverses of two matrices. Linear Multilinear Algebra, 2020, doi:10.1080/03081087.2019.1627279.

[91] Y. Tian. Miscellaneous equalities for idempotent matrices with applications. Open Math., 2020, doi:10.1515/math2020-0147. 
[92] Y. Tian. Miscellaneous reverse order laws for generalized inverses of matrix products with applications. Adv. Oper. Theory, 2020, doi:10.1007/s43036-020-00072-8.

[93] Y. Tian. Two groups of mixed reverse order laws for generalized inverses of two and three matrix products, submitted.

[94] Y. Tian, S. Cheng. The maximal and minimal ranks of $A-B X C$ with applications. New York J. Math. 9(2003), 345-362.

[95] Y. Tian, S. Cheng. Some identities for Moore-Penrose inverses of matrix products. Linear Multilinear Algebra 52(2004), 405-420.

[96] Y. Tian, B. Jiang. Matrix rank/inertia formulas for least-squares solutions with statistical applications. Special Matrices 4(2016), 130-140.

[97] Y. Tian, B. Jiang. Quadratic properties of least-squares solutions of linear matrix equations with statistical applications. Comput. Statist. 32(2017), 1645-1663.

[98] Y. Tian, B. Jiang. Closed-form formulas for calculating the max-min ranks of a triple matrix product composed by generalized inverses. Comp. Appl. Math. 37(2018), 5876-5919.

[99] Y. Tian, Y. Liu. On a group of mixed-type reverse-order laws for generalized inverses of a triple matrix product with applications. Electron. J. Linear Algebra 16(2007), 73-89.

[100] Y. Tian, G.P.H. Styan. Rank equalities for idempotent and involutory matrices. Linear Algebra Appl. 335(2001), 101-117.

[101] Y. Tian, T. Takane. On common generalized inverses of a pair of matrices. Linear Multilinear Algebra 54(2006), $195-209$.

[102] Y. Tian, Y. Wang. Expansion formulas for orthogonal projectors onto ranges of row block matrices. J. Math. Res. Appl. 34(2014), 147-154.

[103] G. Trenkler. Problem 31-7: On the product of orthogonal projectors. IMAGE, Bull. Internat. Linear Algebra Soc. 31(2003), 43.

[104] G. Verbeke, G. Molenberghs. Linear Mixed Models for Longitudinal Data. Springer, New York, 2000.

[105] M. Wang, M. Wei, Z. Jia. Mixed-type reverse-order law of $(A B)^{(1,3)}$. Linear Algebra Appl. 430(2009), $1691-1699$.

[106] H.J. Werner. When is $B^{-} A^{-}$a generalized inverse of $A B$ ? Linear Algebra Appl. 210(1994), 255-263.

[107] E.A. Wibker, R.B. Howe, J.D. Gilbert. Explicit solutions to the reverse order law $(A B)^{+}=B_{m r}^{-} A_{l r}^{-}$. Linear Algebra Appl. 25(1979), 107-114.

[108] Z. Xiong, Y. Qin. On reverse order law for $\{1,3,4\}$-inverse of two matrix product (in Chinese). J. Lanzhou Univ., Natur. Sci. 46(2010), 187-189.

[109] Z. Xiong, Y. Qin. A note on the reverse order law for reflexive generalized inverse of multiple matrix products. Appl. Math. Comput. 219(2013), 4255-4265.

[110] Z. Xiong, B. Zheng. The reverse order laws for $\{1,2,3\}$ - and $\{1,2,4\}$-inverses of a two-matrix product. Applied Math. Lett. 21(2008), 649-655.

[111] H. Yanai, K. Takeuchi, Y. Takane. Projection Matrices, Generalized Inverse Matrices, and Singular Value Decomposition. Springer, New York, 2011.

[112] H. Yang, X. Liu. Mixed-type reverse-order Laws of $(A B)^{(1,2,3)}$ and $(A B)^{(1,2,4)}$. Appl. Math. Comput. 217(2011), 10361-10367.

[113] H. Zhang, F. Lu. Mixed-type reverse order laws for generalized inverses over Hilbert space. Applied Math. 8(2017), 637-644.

[114] H. Zhang, C. Deng. Mixed-type reverse order laws associated to \{1,3,4\}-inverse. J. Math. Res. Appl. 39(2019), 529-539.

[115] B. Zheng, Z. Xiong. The reverse order laws for $\{1,2,3\}$ - and $\{1,2,4\}$-inverses of multiple matrix products. Linear Multilinear Algebra 58(2010), 765-782.

[116] Y. Zhao. Approximation theory of matrix rank minimization and its application to quadratic equations. Linear Algebra Appl. 437(2012), 77-93. 